\title{
Enabling $N$-to- $C$ Ser/Thr Ligation for Convergent Protein Synthesis via Combining Chemical Ligation Approaches
}

\author{
Chi Lung Lee, Han Liu, Clarence T. T. Wong, Hoi Yee Chow and Xuechen Li* \\ Department of Chemistry, The State Key Laboratory of Synthetic Chemistry, The University \\ of Hong Kong, Pokfulam, Hong Kong SAR, China \\ Email:xuechenl@hku.hk
}

\section{$\underline{\text { Supplementary Information }}$}

$\begin{array}{ll}\text { I. General Information } & \text { S2 }\end{array}$

II. General Experimental Procedures $\quad$ S3

III. Synthesis of C-terminus '1' L-Amino salicylaldehyde semicarbazone S6

ester hydrochloride $\left(\mathrm{HCl} \cdot \mathrm{H}_{2} \mathrm{~N}\right.$-Xaa-CO-SAL $\left.{ }^{\text {off }}\right)$ for $\mathrm{n}+1$ reaction

IV. Synthesis of model peptide $\mathrm{SAL}^{\text {on }}$ and $\mathrm{SAL}^{\text {off }}$ ester by $\mathrm{n}+1$ strategy $\quad \mathrm{S} 10$

V. Epimerization study of peptide ester generated by $n+1$ strategy $\quad$ S28

VI. Model $N$-to- $C$ STL study between peptide SAL ${ }^{\text {on }}$ ester and peptide S31

$\mathrm{SAL}^{\text {off }}$ ester to generate ligated peptide $\mathrm{SAL}^{\text {on }}$ ester through the use of pyruvic acid

$\begin{array}{ll}\text { VII. Synthesis of peptide 5, } 7 \text { and } 8 & \text { S49 }\end{array}$

VIII. Application of $N$-to- $C$ strategy on convergent STL S53

IX. One-pot Three-segment STL and NCL $\quad$ S62

X. Total synthesis of glycosylated IL-25 by N-to-C STL strategy and S66

One-pot Three-segment STL and NCL

XI. ${ }^{1} \mathrm{H}$ and ${ }^{13} \mathrm{C}$ NMR spectra $\quad$ S94

$\begin{array}{lr}\text { XII. References } & \text { S108 }\end{array}$ 


\section{General Information}

\section{A. Materials and methods and abbreviations}

All commercial materials (Aldrich, Chemimpex, Fluka and GL Biochem) were used without further purification. All solvents were reagent grade or HPLC grade (RCI or DUKSAN). Dry dichloromethane $\left(\mathrm{CH}_{2} \mathrm{Cl}_{2}\right)$ was distilled from calcium hydride $\left(\mathrm{CaH}_{2}\right)$. All reversed-phase (RP) high-performance liquid chromatography (HPLC) separations involved a mobile phase of $0.1 \%$ trifluoroacetic acid (TFA) $(v / v)$ in acetonitrile $\left(\mathrm{CH}_{3} \mathrm{CN}\right) / 0.1 \%$ TFA $(v / v)$ in water $\left(\mathrm{H}_{2} \mathrm{O}\right)$ were performed with a Waters HPLC system equipped with a photodiode array detector (Waters 2996) using a Vydac $214 \mathrm{TP}^{\mathrm{TM}} \mathrm{C} 4$ column $(5 \mu \mathrm{m}, 300 \AA, 4.6 \mathrm{x} 250 \mathrm{~mm})$ at a flow rate of 0.6 $\mathrm{mL} / \mathrm{min}$ for analytical HPLC and Vydac $214 \mathrm{TP}^{\mathrm{TM}} \mathrm{C} 4$ column $(10 \mu \mathrm{m}, 300 \AA, 22 \mathrm{x}$ $250 \mathrm{~mm})$ or Vydac $218 \mathrm{TP}^{\mathrm{TM}} \mathrm{C} 18$ column $(10 \mu \mathrm{m}, 300 \AA, 22 \times 250 \mathrm{~mm})$ at a flow rate of $10 \mathrm{~mL} / \mathrm{min}$ for preparative HPLC. Low-resolution mass spectral (MS) analyses were performed with a Waters 3100 mass spectrometer using electrospray ionization (ESI, in positive mode unless otherwise specified). The results were analyzed with Waters Empower software. Calculated masses were based upon the most abundant isotope of a given ion. Analytical TLC was performed on E. Merck silica gel 60 F254 plates and visualized under UV light $(254 \mathrm{~nm})$ or by staining with ninhydrin or $5 \%$ sulfuric acid in methanol. Silica flash column chromatography was performed on E. Merck 230-400 mesh silica gel $60 .{ }^{1} \mathrm{H}$ and ${ }^{13} \mathrm{C}$ nuclear magnetic resonance (NMR) spectra were recorded at $298 \mathrm{~K}$ on Bruker Avance DRX 300 FT-NMR Spectrometer at $75 \mathrm{MHz}$ for ${ }^{13} \mathrm{C}$ NMR or Bruker Avance DRX 400 FT-NMR spectrometer at 400 $\mathrm{MHz}$ for ${ }^{1} \mathrm{H}$ NMR and $100 \mathrm{MHz}$ for ${ }^{13} \mathrm{C}$ NMR or Bruker Avance DRX 600 FT-NMR spectrometer at $150 \mathrm{MHz}$ for ${ }^{13} \mathrm{C} \mathrm{NMR}$. Chemical shifts are reported in parts per million (ppm) and are referenced to solvent residual signals: $\mathrm{CDCl}_{3}(\delta 7.26[1 \mathrm{H}]) .1 \mathrm{H}$ NMR data is reported as chemical shift $(\delta)$, relative integral, multiplicity ( $\mathrm{s}=$ singlet, $\mathrm{d}=$ doublet, $\mathrm{t}=$ triplet, $\mathrm{dd}=$ doublet of doublets, $\mathrm{td}=$ triplet of doublets $)$, coupling constant $(\mathrm{J} \mathrm{Hz})$. LCMS = Liquid chromatography mass-spectrometry; PG = protecting groups; $\mathrm{SAL}=$ salicylaldehyde; $\mathrm{DMF}=$ dimethylformamide; $\mathrm{TIPS}=$ triisopropylsilane. 


\section{General Experimental Procedures}

\section{A. Fmoc-based Solid-phase Peptide Synthesis (SPPS)}

The solid phase peptide synthesis of peptides/glycopeptides was carried out manually using 2-chlorotrityl resin (GL Biochem, loading: $\sim 0.5 \mathrm{mmol} / \mathrm{g}$ ) unless otherwise specified. 2-chloro-trityl chloride resin was swollen in dry $\mathrm{CH}_{2} \mathrm{Cl}_{2}$ for 30 min then washed with $\mathrm{CH}_{2} \mathrm{Cl}_{2}(5 \times 3 \mathrm{~mL})$. A solution of FmocHN-Xaa-COOH (4.0 equiv. relative to resin capacity) and DIEA (8.0 equiv. relative to resin capacity) in $\mathrm{CH}_{2} \mathrm{Cl}_{2}$ was added and the resin shaken at room temperature (r. t.) for $2 \mathrm{~h}$. The resin was washed with DMF $(5 \times 3 \mathrm{~mL})$ and $\mathrm{CH}_{2} \mathrm{Cl}_{2}(5 \times 3 \mathrm{~mL})$. The resin was treated with a solution of $\mathrm{CH}_{2} \mathrm{Cl}_{2} / \mathrm{CH}_{3} \mathrm{OH} / \mathrm{DIEA}(17: 2: 1, v / v / v, 3 \mathrm{~mL})$ for $1 \mathrm{~h}$ and washed with DMF $(5 \times 3 \mathrm{~mL}), \mathrm{CH}_{2} \mathrm{Cl}_{2}(5 \times 3 \mathrm{~mL})$, and DMF $(5 \times 3 \mathrm{~mL})$. The resin was subsequently submitted to iterative peptide assembly (Fmoc-SPPS). The following Fmoc amino acids and Boc amino acids from GL Biochem were employed: FmocHN-Ala-COOH, FmocHN-Cys(Trt)-COOH, FmocHN-Cys(S $\left.{ }^{t} \mathrm{Bu}\right)-\mathrm{COOH}$, FmocHN-Asp(Ot $\left.\mathrm{Bu}\right)-\mathrm{COOH}$, FmocHN-Glu(O $\left.{ }^{t} \mathrm{Bu}\right)-\mathrm{COOH}, \quad$ FmocHN-Phe-COOH, FmocHN-Gly-COOH, FmocHN-His(Trt)-COOH, FmocHN-Ile-COOH, FmocHN-Lys(Boc)-COOH, FmocHN-Leu-COOH, FmocHN-Met-COOH, FmocHN-Asn(Trt)-COOH, FmocHN-Pro-COOH, FmocHN-Gln(Trt)-COOH, FmocHN-Arg(Pbf)-COOH, FmocHN-Ser $\left({ }^{t} \mathrm{Bu}\right)-\mathrm{COOH}, \quad$ FmocHN-Thr $\left({ }^{t} \mathrm{Bu}\right)-\mathrm{COOH}, \quad$ FmocHN-Val-COOH, FmocHN-Trp(Boc)-COOH, FmocHN-Tyr $\left({ }^{t} \mathrm{Bu}\right)-\mathrm{COOH}, \quad$ BocHN-Ser( $\left.{ }^{t} \mathrm{Bu}\right)-\mathrm{COOH}$, BocHN-Thr $\left({ }^{t} \mathrm{Bu}\right)-\mathrm{COOH}$, BocHN-Tyr $\left({ }^{\circ} \mathrm{Bu}\right)-\mathrm{COOH}$ and BocN-Thz-COOH. The following pseudoproline dipeptides from Chemimpex were used: FmocHN-Gln-Thr( $\left.\Psi^{\mathrm{Me}, \mathrm{Me}} \mathrm{Pro}\right)-\mathrm{COOH}$ and FmocHN-Asn-Ser( $\left.\Psi^{\mathrm{Me}, \mathrm{Me}} \mathrm{Pro}\right)-\mathrm{COOH}$. The glycosyl amino acid FmocHN-Asn(GlcNAc 4$)-\mathrm{COOH}$ was obtained by synthesis. The removal of Fmoc group was executed using a deblock solution of $20 \%$ piperidine in DMF at room temperature for $20 \mathrm{~min}$. The resin was washed with DMF $(5 \times 3 \mathrm{~mL})$, $\mathrm{CH}_{2} \mathrm{Cl}_{2}(5 \times 3 \mathrm{~mL})$, and DMF $(5 \times 3 \mathrm{~mL})$. For the coupling step, a solution of Fmoc protected amino acid or Boc protected amino acid (2.0 equiv. according to the resin capacity), HATU (2.0 equiv.) and DIEA (5.0 equiv.) in DMF was gently agitated with the resin at room temperature for $40 \mathrm{~min}$. The resin was washed with DMF $(5 \times 3$ 
$\mathrm{mL}), \mathrm{CH}_{2} \mathrm{Cl}_{2}(5 \times 3 \mathrm{~mL})$, and DMF $(5 \times 3 \mathrm{~mL})$. This procedure was repeated twice for coupling each amino acid.

\section{B. Cleavage of crude protected peptide bearing the free carboxylic acid at the C-terminus from resin with Cocktail A}

The on-resin fully protected peptidyl acid, obtained as described in the previous section, was subjected to mild acidic cleavage cocktail (5 - $10 \mathrm{~mL})$ of $\mathrm{CH}_{2} \mathrm{Cl}_{2} / \mathrm{AcOH} /$ trifluoroethanol $(8 / 1 / 1, v / v / v), 3$ times for $60 \mathrm{~min}$ each. Following filtration, the resulting cleavage solutions were combined and concentrated to give crude protected peptide bearing the free carboxylic acid at the $\mathrm{C}$-terminus.

\section{Synthesis of C-terminus ' 1 ' L-Amino salicylaldehyde semicarbazone ester $\underline{\text { hydrochloride }\left(\mathrm{HCl} \cdot \mathrm{H}_{2} N \text {-Xaa-CO-SAL }{ }^{\text {off }}\right)}$}

To a solution of fully protected Boc amino acid, $\mathrm{BocHN}-\mathrm{Xaa}(\mathrm{PG})-\mathrm{COOH}$, (1.0 equiv.) in $\mathrm{CH}_{2} \mathrm{Cl}_{2}$ at room temperature, $N$-(3-dimethylaminopropyl)- $N$ '-ethylcarbodiimide hydrochloride (EDCI) (3.0 equiv.) and 4-Dimethylaminopyridine (DMAP) (0.1 equiv.) were added, followed by salicylaldehyde semicarbazone (1.0 equiv.). The reaction mixture was stirred overnight at room temperature and concentrated in vacuo. Purification by silica gel chromatography $\left(\mathrm{CH}_{2} \mathrm{Cl}_{2} / \mathrm{EtOAc}, 2: 1\right)$ gave $B o c H N-\mathrm{Xaa}(\mathrm{PG})-C O-\mathrm{SAL}^{\text {off }}$ as a white solid. This compound was treated with a solution of $\mathrm{HCl} /$ dioxane (4 M) and stirred for $2 \mathrm{~h}$. The solvent was removed by blowing a stream of condensed air and the residue triturated with diethyl ether $(\times 2)$ and dried under vacuum to afford $\mathrm{HCl} \cdot \mathrm{H}_{2} \mathrm{~N}$-Xaa-CO-SAL ${ }^{\text {off }}$ as a white solid. Without purification, this salt was subjected to the $n+1$ reaction.

\section{Synthesis of $C$-terminus Peptide SAL ${ }^{\text {off }}$ esters using $n+1$ strategy}

The fully protected peptidyl acid (1.0 equiv.), obtained as described in the previous section $\mathrm{A}$ and $\mathrm{B}$, was dissolved in $\mathrm{CHCl}_{3} /$ trifluoroethanol $(15 \mathrm{mM}, 3 / 1, v / v)$, and $N$-(3-dimethylaminopropyl)- $N$ '-ethylcarbodiimide $\quad$ (EDC) (3.0 equiv.) and Hydroxy-3,4-dihydro-4-oxo-1,2,3-benzotriazine (HOOBt) (3.0 equiv.) were then 
added. After $5 \mathrm{~min}$, the corresponding amino L-Amino salicylaldehyde semicarbazone ester hydrochloride $\left(\mathrm{HCl} \cdot \mathrm{H}_{2} \mathrm{~N}\right.$-Xaa- $\left.\mathrm{CO}-\mathrm{SAL}^{\text {off }}\right)(3.0$ equiv.), obtained as described in the previous section, was added, and the reaction mixture was stirred for $3 \mathrm{~h}$ to form the crude protected C-terminal peptide $\mathrm{SAL}^{\text {off }}$ ester.

\section{E. Preparation of Native chemical ligation buffer}

Native chemical ligation (NCL) buffer was freshly prepared prior to the reaction by dissolving $\mathrm{Na}_{2} \mathrm{HPO}_{4}(56.6 \mathrm{mg}, 0.2 \mathrm{M})$ and guanidine $(\mathrm{Gn}) \cdot \mathrm{HCl}(1.146 \mathrm{~g}, 6 \mathrm{M})$ in water. Tris(2-carboxyethyl)phosphine hydrochloride (TCEP.HCl) $(10.8 \mathrm{mg}, 0.02 \mathrm{M})$ was then added, and the mixture was diluted to $2.0 \mathrm{~mL}$. After complete solubilization, the $\mathrm{pH}$ was brought to $\sim 7.0$ by the addition of a $\mathrm{NaOH}$ solution $(5.0 \mathrm{M})$. 4-mercaptophenylacetic acid (MPAA) $(67 \mathrm{mg}, 0.2 \mathrm{M})$ was then added and the $\mathrm{pH}$ of the mixture was adjusted to $6.8-7.2$ with $\mathrm{NaOH}(5.0 \mathrm{M})$. The solution was sonicated and sparged with Ar for 30 min before use.

\section{$\underline{\text { F. Preparation of hydrazine 2-chlorotrityl chloride resin }}$}

2-chlorotrityl chloride resin $(1 \mathrm{~g}$, loading $=\sim 0.5 \mathrm{mmol} / \mathrm{g})$ was swelled in $10 \mathrm{~mL}$ $\mathrm{CH}_{2} \mathrm{Cl}_{2} / \mathrm{DMF}(1 / 1, v / v)$. Then $10 \mathrm{~mL} \mathrm{NH}_{2} \mathrm{NH}_{2} \cdot \mathrm{H}_{2} \mathrm{O} / \mathrm{DMF}(1 / 20, v / v)$ were added. The reaction was conducted for $30 \mathrm{~min} .10 \mathrm{~mL}$ of methanol/DMF $(1 / 20, v / v)$ was added to quench the remaining 2-chlorotrityl chloride resin. After $30 \mathrm{~min}$, the resin was washed with DMF and $\mathrm{CH}_{2} \mathrm{Cl}_{2}$ and kept under high vacuum for $2 \mathrm{~h}$ and ready for iterative peptide assembly (Fmoc-SPPS). 
III. Synthesis of C-terminus ' 1 ' L-Amino salicylaldehyde semicarbazone ester hydrochloride $\left(\mathrm{HCl} \cdot \mathrm{H}_{2} \mathrm{~N}-\mathrm{Xaa}-\mathrm{CO}-\mathrm{SAL}{ }^{\text {off }}\right)$ for $\underline{n+1 \text { reaction }}$

$N$-(tert-Butoxycarbonyl)-L-Alanine salicylaldehyde semicarbazone ester (BocHN-Ala-CO-SAL off)

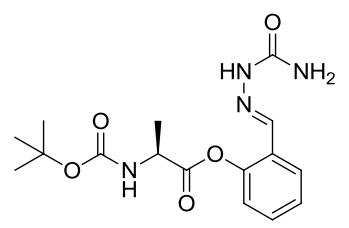

BocHN-Ala-CO-SAL ${ }^{\text {off }}(157.2 \mathrm{mg}, 80 \%$ yield) was obtained according to the general procedure $\mathrm{C}$ with the use of $\mathrm{BocHN}$-Ala-COOH $(106.1 \mathrm{mg}, 561.4 \mu \mathrm{mol})$. This compound was then treated with a solution of $\mathrm{HCl} /$ dioxane $(4 \mathrm{M})$ and stirred for $2 \mathrm{~h}$. The solvent was removed by blowing a stream of condensed air and the residue triturated with diethyl ether $(\times 2)$ and dried under vacuum to afford $\mathrm{HCl} \cdot \mathrm{H}_{2} \mathrm{~N}$-Ala-CO-SAL ${ }^{\text {off }}(\mathrm{A})$ as a white solid. Without any purification, this salt was subjected to the $\mathrm{n}+1$ reaction. ${ }^{1} \mathrm{H}$ NMR $\left(400 \mathrm{MHz} \mathrm{CDCl}_{3}\right) \delta 10.15(1 \mathrm{H}, \mathrm{s}), 7.99(1 \mathrm{H}$, s), $7.87(1 \mathrm{H}, \mathrm{d}, J=7.6 \mathrm{~Hz}), 7.37(1 \mathrm{H}, \mathrm{t}, J=7.2 \mathrm{~Hz}), 7.25(1 \mathrm{H}, \mathrm{t}, J=7.5 \mathrm{~Hz}), 7.08(1 \mathrm{H}$, $\mathrm{d}, J=7.9 \mathrm{~Hz}), 6.15(2 \mathrm{H}, \mathrm{br}), 5.55(1 \mathrm{H}, \mathrm{br}), 4.60(1 \mathrm{H}, \mathrm{br}), 1.57(3 \mathrm{H}, \mathrm{d}, J=7.2 \mathrm{~Hz})$, $1.46(9 \mathrm{H}, \mathrm{s}){ }^{13} \mathrm{C} \mathrm{NMR}\left(100 \mathrm{MHz}, \mathrm{CDCl}_{3}\right) \delta 172.5,158.5,155.9,149.4,137.1,130.8$, 126.7, 126.5, 122.8, 80.8, 49.8, 28.7, 18.3 ESI-MS calcd. for $\mathrm{C}_{16} \mathrm{H}_{23} \mathrm{~N}_{4} \mathrm{O}_{5}[\mathrm{M}+\mathrm{H}]^{+}$ $m / z=351.17$, found 351.22 .

$N$-(tert-Butoxycarbonyl)-L-Glycine salicylaldehyde semicarbazone ester (BocHN-Gly-CO-SAL ${ }^{\text {off }}$ )

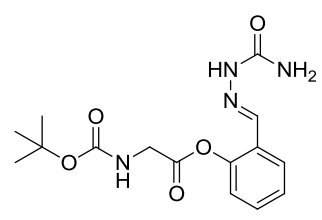


BocHN-Gly-CO-SAL ${ }^{\text {off }}$ (130.5 mg, $58 \%$ yield) was obtained according to the general procedure $\mathrm{C}$ with the use of $\mathrm{BocHN}-\mathrm{Gly}-\mathrm{COOH}(117.2 \mathrm{mg}, 669.7 \mu \mathrm{mol})$. This compound was then treated with a solution of $\mathrm{HCl} /$ dioxane $(4 \mathrm{M})$ and stirred for $2 \mathrm{~h}$. The solvent was removed by blowing a stream of condensed air and the residue triturated with diethyl ether $\left(\begin{array}{ll}\times & 2\end{array}\right)$ and dried under vacuum to afford $\mathrm{HCl} \cdot \mathrm{H}_{2} \mathrm{~N}$-Gly-CO-SAL ${ }^{\text {off }}(\mathrm{G})$ as a white solid. Without any purification, this salt was subjected to the $\mathrm{n}+1$ reaction. ${ }^{1} \mathrm{H}$ NMR $\left(400 \mathrm{MHz}, \mathrm{CDCl}_{3}\right) \delta 10.23(1 \mathrm{H}, \mathrm{s}), 7.95(1 \mathrm{H}$, s), $7.83(1 \mathrm{H}, \mathrm{d}, J=7.6 \mathrm{~Hz}), 7.36(1 \mathrm{H}, \mathrm{t}, J=7.6 \mathrm{~Hz}), 7.24(1 \mathrm{H}, \mathrm{t}, J=7.5 \mathrm{~Hz}), 7.10(1 \mathrm{H}$, $\mathrm{d}, J=8.1 \mathrm{~Hz}), 6.18(2 \mathrm{H}, \mathrm{br}), 5.68(1 \mathrm{H}, \mathrm{s}), 4.22(2 \mathrm{H}, \mathrm{m}), 1.44(9 \mathrm{H}, \mathrm{s}){ }^{13} \mathrm{C}$ NMR $(75$ $\left.\mathrm{MHz}, \mathrm{CDCl}_{3}\right) \delta 169.7,158.2,156.4,149.3,137.4,131.0,126.9,126.6,122.9,80.9$, 42.9, 28.7 ESI-MS calcd. for $\mathrm{C}_{15} \mathrm{H}_{21} \mathrm{~N}_{4} \mathrm{O}_{5}[\mathrm{M}+\mathrm{H}]^{+} m / z=337.15$, found 337.19.

\section{$N$-(tert-Butoxycarbonyl)-L-Leucine salicylaldehyde semicarbazone ester (BocHN-Leu-CO-SAL $\left.{ }^{\text {off }}\right)$}

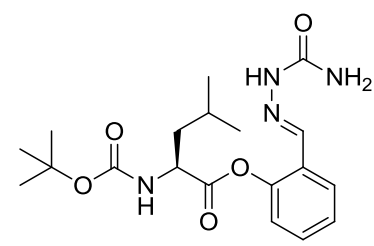

BocHN-Leu-CO-SAL ${ }^{\text {off }}$ (107.5 mg, 64\% yield) was obtained according to the general procedure $\mathrm{C}$ with the use of BocHN-Leu-COOH (99.0 mg, $428.6 \mu \mathrm{mol})$. This compound was then treated with a solution of $\mathrm{HCl} /$ dioxane $(4 \mathrm{M})$ and stirred for $2 \mathrm{~h}$. The solvent was removed by blowing a stream of condensed air and the residue triturated with diethyl ether $\left(\begin{array}{l}\times\end{array}\right)$ and dried under vacuum to afford $\mathrm{HCl} \cdot \mathrm{H}_{2} \mathrm{~N}$-Leu-CO-SAL ${ }^{\text {off }}(\mathrm{L})$ as a white solid. Without any purification, this salt was subjected to the $\mathrm{n}+1$ reaction. ${ }^{1} \mathrm{H}$ NMR $\left(400 \mathrm{MHz}, \mathrm{CDCl}_{3}\right) \delta 9.31(1 \mathrm{H}, \mathrm{s}), 8.09(1 \mathrm{H}, \mathrm{s})$, $7.89(1 \mathrm{H}, \mathrm{dd}, J=7.8,1.2 \mathrm{~Hz}), 7.41(1 \mathrm{H}, \mathrm{td}, J=8.0,1.6 \mathrm{~Hz}), 7.29(1 \mathrm{H}, \mathrm{t}, J=7.6 \mathrm{~Hz})$, $7.08(1 \mathrm{H}, \mathrm{d}, J=8.1 \mathrm{~Hz}), 6.17(2 \mathrm{H}, \mathrm{br}), 5.14(1 \mathrm{H}, \mathrm{d}, J=7.7 \mathrm{~Hz}), 4.51(1 \mathrm{H}, \mathrm{m}), 1.83$ $(1 \mathrm{H}, \mathrm{m}), 1.69(1 \mathrm{H}, \mathrm{m}), 1.45(9 \mathrm{H}, \mathrm{s}), 1.03(6 \mathrm{H}, \mathrm{m}){ }^{13} \mathrm{C} \mathrm{NMR}\left(75 \mathrm{MHz}, \mathrm{CDCl}_{3}\right) \delta 172.6$, 157.6, 156.2, 149.6, 137.4, 130.9, 126.8, 126.4, 122.7, 80.9, 52.8, 41.2, 28.7, 25.3, 23.3, 22.1 ESI-MS calcd. for $\mathrm{C}_{19} \mathrm{H}_{29} \mathrm{~N}_{4} \mathrm{O}_{5}[\mathrm{M}+\mathrm{H}]^{+} \mathrm{m} / z=393.21$, found 393.37 


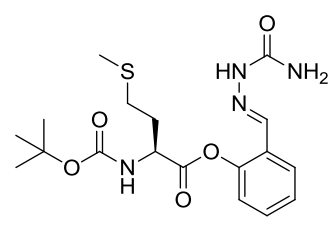

BocHN-Met-CO-SAL ${ }^{\text {off }}$ (84.9 mg, $48 \%$ yield) was obtained according to the general procedure $\mathrm{C}$ with the use of BocHN-Met-COOH (107.5 mg, $431.7 \mu \mathrm{mol})$. This compound was then treated with a solution of $\mathrm{HCl} /$ dioxane $(4 \mathrm{M})$ and stirred for $2 \mathrm{~h}$. The solvent was removed by blowing a stream of condensed air and the residue triturated with diethyl ether ( $\times 2$ ) and dried under vacuum to afford $\mathrm{HCl} \cdot \mathrm{H}_{2} \mathrm{~N}$-Met-CO-SAL ${ }^{\text {off }}(\mathrm{M})$ as a white solid. Without any purification, this salt was subjected to the $\mathrm{n}+1$ reaction. ${ }^{1} \mathrm{H} \mathrm{NMR}\left(400 \mathrm{MHz}, \mathrm{CDCl}_{3}\right) \delta 9.96(1 \mathrm{H}, \mathrm{s}), 8.02(1 \mathrm{H}, \mathrm{s})$, $7.88(1 \mathrm{H}, \mathrm{d}, J=7.7 \mathrm{~Hz}), 7.40(1 \mathrm{H}, \mathrm{t}, J=8.2 \mathrm{~Hz}), 7.29(1 \mathrm{H}, \mathrm{t}, J=7.6 \mathrm{~Hz}), 7.11(1 \mathrm{H}, \mathrm{d}$, $J=8.0 \mathrm{~Hz}), 6.23(2 \mathrm{H}, \mathrm{br}), 5.51(1 \mathrm{H}, \mathrm{d}, J=7.8 \mathrm{~Hz}), 4.70(1 \mathrm{H}, \mathrm{m}), 2.68(2 \mathrm{H}, \mathrm{m}), 2.34$ $(1 \mathrm{H}, \mathrm{m}), 2.17(4 \mathrm{H}, \mathrm{m}), 1.46(9 \mathrm{H}, \mathrm{s}){ }^{13} \mathrm{C} \mathrm{NMR}\left(150 \mathrm{MHz}, \mathrm{CDCl}_{3}\right) \delta 171.2,157.2$, 155.8, 149.1, 136.7, 130.6, 126.6, 126.3, 126.1, 122.4, 80.8, 53.1, 31.2, 30.2, 28.4, 15.6 ESI-MS calcd. for $\mathrm{C}_{18} \mathrm{H}_{27} \mathrm{~N}_{4} \mathrm{O}_{5} \mathrm{~S}[\mathrm{M}+\mathrm{H}]^{+} m / z=411.17$, found 411.30.

$N$-(tert-Butoxycarbonyl)-O-tert-butyl-L-Serine salicylaldehyde semicarbazone ester $\left(B o c H N-S e r\left(O^{t} \mathrm{Bu}\right)-C O-S A L{ }^{\text {off }}\right)$

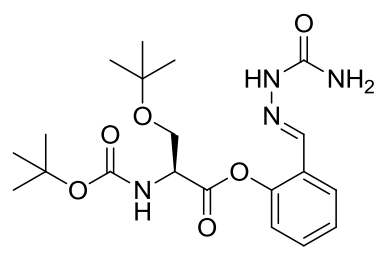

$\boldsymbol{B o c H N}-\operatorname{Ser}\left(\mathbf{O}^{t} \mathbf{B u}\right)-\boldsymbol{C O}$-SAL ${ }^{\text {off }}$ (66.8 $\mathrm{mg}, 40 \%$ yield) was obtained according to the general procedure $\mathrm{C}$ with the use of $\mathrm{BocHN}-\mathrm{Ser}\left(\mathrm{O}^{t} \mathrm{Bu}\right)-\mathrm{COOH}(103.3 \mathrm{mg}, 395.7$ $\mu \mathrm{mol})$. This compound was then treated with a solution of $\mathrm{HCl} / \mathrm{dioxane}(4 \mathrm{M})$ and stirred for $2 \mathrm{~h}$. The solvent was removed by blowing a stream of condensed air and the residue triturated with diethyl ether $(\times 2)$ and dried under vacuum to afford 
$\mathrm{HCl} \cdot \mathrm{H}_{2} \mathrm{~N}$-Ser-CO-SAL ${ }^{\text {off }}(\mathrm{S})$ as a white solid. Without any purification, this salt was subjected to the $\mathrm{n}+1$ reaction. ${ }^{1} \mathrm{H}$ NMR $\left(400 \mathrm{MHz}, \mathrm{CDCl}_{3}\right) \delta 9.73(1 \mathrm{H}, \mathrm{s}), 8.03(1 \mathrm{H}, \mathrm{s})$, $7.87(1 \mathrm{H}, \mathrm{d}, J=7.7 \mathrm{~Hz}), 7.41(1 \mathrm{H}, \mathrm{td}, J=7.9,1.5 \mathrm{~Hz}), 7.28(1 \mathrm{H}, \mathrm{t}, J=7.4 \mathrm{~Hz}), 7.08$ $(1 \mathrm{H}, \mathrm{d}, J=8.1 \mathrm{~Hz}), 6.31(2 \mathrm{H}, \mathrm{br}), 5.61(1 \mathrm{H}, \mathrm{d}, J=8.6 \mathrm{~Hz}), 4.66(1 \mathrm{H}, \mathrm{d}, J=8.2 \mathrm{~Hz})$, $4.02(1 \mathrm{H}, \mathrm{dd}, J=9.0,2.8 \mathrm{~Hz}), 3.70(1 \mathrm{H}, \mathrm{dd}, \mathrm{J}=9.1,3.0 \mathrm{~Hz}), 1.46(9 \mathrm{H}, \mathrm{s}), 1.22(9 \mathrm{H}, \mathrm{s})$ ${ }^{13} \mathrm{C}$ NMR (100 MHz, $\left.\mathrm{CDCl}_{3}\right) \delta 170.2,157.7,156.3,149.6,137.3,130.8,126.7,126.5$, 122.9, 80.8, 74.1, 62.5, 54.9, 28.7, 27.7 ESI-MS calcd. for $\mathrm{C}_{20} \mathrm{H}_{31} \mathrm{~N}_{4} \mathrm{O}_{6}[\mathrm{M}+\mathrm{H}]^{+}$ $m / z=423.22$, found 423.30 .

\section{$N$-(tert-Butoxycarbonyl)-L-Valine salicylaldehyde semicarbazone ester (BocHN-Val-CO-SAL $\left.{ }^{\text {off }}\right)$}

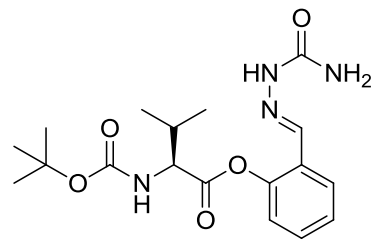

BocHN-Val-CO-SAL ${ }^{\text {off }}$ (45.2 $\mathrm{mg}, 24 \%$ yield) was obtained according to the general procedure $\mathrm{C}$ with the use of BocHN-Val-COOH (108.1 mg, $498.2 \mu \mathrm{mol})$. This compound was then treated with a solution of $\mathrm{HCl} /$ dioxane $(4 \mathrm{M})$ and stirred for $2 \mathrm{~h}$. The solvent was removed by blowing a stream of condensed air and the residue triturated with diethyl ether $\left(\begin{array}{ll}\times & 2\end{array}\right)$ and dried under vacuum to afford $\mathrm{HCl} \cdot \mathrm{H}_{2} \mathrm{~N}$-Val-CO-SAL ${ }^{\text {off }}(\mathrm{V})$ as a white solid. Without any purification, this salt was subjected to the $\mathrm{n}+1$ reaction. ${ }^{1} \mathrm{H}$ NMR $\left(400 \mathrm{MHz}, \mathrm{CDCl}_{3}\right) \delta 9.45(1 \mathrm{H}, \mathrm{s}), 8.09(1 \mathrm{H}, \mathrm{s})$, $7.89(1 \mathrm{H}, \mathrm{dd}, J=7.8,1.1 \mathrm{~Hz}), 7.42(1 \mathrm{H}, \mathrm{td}, J=7.9,1.5 \mathrm{~Hz}), 7.29(1 \mathrm{H}, \mathrm{t}, J=7.6 \mathrm{~Hz})$, $7.06(1 \mathrm{H}, \mathrm{d}, J=8.5 \mathrm{~Hz}), 6.20(2 \mathrm{H}, \mathrm{br}), 5.10(1 \mathrm{H}, \mathrm{d}, J=8.2 \mathrm{~Hz}), 4.40(1 \mathrm{H}, \mathrm{dd}, \mathrm{J}=8.1$, $5.3 \mathrm{~Hz}), 2.32(1 \mathrm{H}, \mathrm{m}), 1.46(9 \mathrm{H}, \mathrm{s}), 1.12(3 \mathrm{H}, \mathrm{d}, J=6.8 \mathrm{~Hz}), 1.06(3 \mathrm{H}, \mathrm{d}, J=6.8 \mathrm{~Hz})$ ${ }^{13} \mathrm{C}$ NMR $\left(75 \mathrm{MHz}, \mathrm{CDCl}_{3}\right) \delta 171.4,158.2,156.4,149.4,137.2,130.7,126.8,126.7$, 126.5, 122.7, 80.7, 59.5, 31.2, 28.7, 19.6, 18.0 ESI-MS calcd. for $\mathrm{C}_{18} \mathrm{H}_{27} \mathrm{~N}_{4} \mathrm{O}_{5}[\mathrm{M}+\mathrm{H}]^{+}$ $m / z=379.43$, found 379.27 .

$N$-(tert-Butoxycarbonyl)-O-tert-butyl-L-Tyrosine salicylaldehyde semicarbazone ester $\left(B o c H N-T y r\left(O^{t} B u\right)-C O-S A L^{o f f}\right)$ 


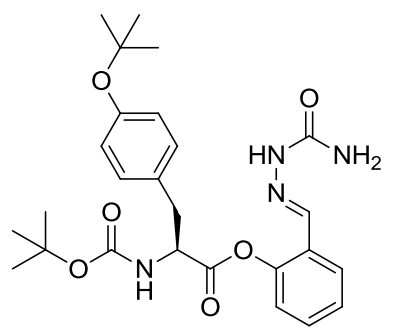

BocHN-Tyr(O' $\left.\mathbf{O}^{t} \mathbf{B u}\right)-C O-S A L^{\text {off }}(115.1 \mathrm{mg}, 68 \%$ yield) was obtained according to the general procedure $\mathrm{C}$ with the use of $B o c H N-\operatorname{Tyr}\left(\mathrm{O}^{t} \mathrm{Bu}\right)-\mathrm{COOH}(114.5 \mathrm{mg}, 339.8$ $\mu \mathrm{mol})$. This compound was then treated with a solution of $\mathrm{HCl} /$ dioxane $(4 \mathrm{M})$ and stirred for $2 \mathrm{~h}$. The solvent was removed by blowing a stream of condensed air and the residue triturated with diethyl ether $(\times 2)$ and dried under vacuum to afford $\mathrm{HCl} \cdot \mathrm{H}_{2} \mathrm{~N}$-Tyr-CO-SAL ${ }^{\text {off }}(\mathrm{Y})$ as a white solid. Without any purification, this salt was subjected to the $\mathrm{n}+1$ reaction. ${ }^{1} \mathrm{H}$ NMR $\left(400 \mathrm{MHz}, \mathrm{CDCl}_{3}\right) \delta 9.44(1 \mathrm{H}, \mathrm{s}), 7.85(1 \mathrm{H}, \mathrm{s})$, $7.36(1 \mathrm{H}, \mathrm{t}, J=7.5 \mathrm{~Hz}), 7.26(1 \mathrm{H}, \mathrm{m}), 7.17(1 \mathrm{H}, \mathrm{d}, J=8.3 \mathrm{~Hz}), 6.98(2 \mathrm{H}, \mathrm{d}, J=8.0$ Hz), $6.89(1 \mathrm{H}, \mathrm{d}, J=8.0 \mathrm{~Hz}), 6.09(2 \mathrm{H}, \mathrm{br}), 5.25(1 \mathrm{H}, \mathrm{d}, J=7.1 \mathrm{~Hz}), 4.76(1 \mathrm{H}, \mathrm{m})$, $3.18(2 \mathrm{H}, \mathrm{m}), 1.42(9 \mathrm{H}, \mathrm{s}), 1.35(9 \mathrm{H}, \mathrm{s}){ }^{13} \mathrm{C} \mathrm{NMR}\left(100 \mathrm{MHz}, \mathrm{CDCl}_{3}\right) \delta 171.4,157.2$, 155.9, 155.0, 149.4, 137.2, 130.8, 130.3, 126.7, 126.6, 126.3, 124.7, 122.7, 80.9, 79.0, 55.3, 37.6, 29.2, 28.7 ESI-MS calcd. for $\mathrm{C}_{26} \mathrm{H}_{35} \mathrm{~N}_{4} \mathrm{O}_{6}[\mathrm{M}+\mathrm{H}]^{+} m / z=499.26$, found 499.21.

\section{Synthesis of model peptide $\mathrm{SAL}^{\text {on }}$ and $\mathrm{SAL}^{\text {off }}$ ester by $\mathrm{n}+1$ strategy}

Peptide 1a: $\mathrm{H}_{2} \mathrm{~N}$-VIGGVGNA-CO-SAL ${ }^{\text {on }}$ ester

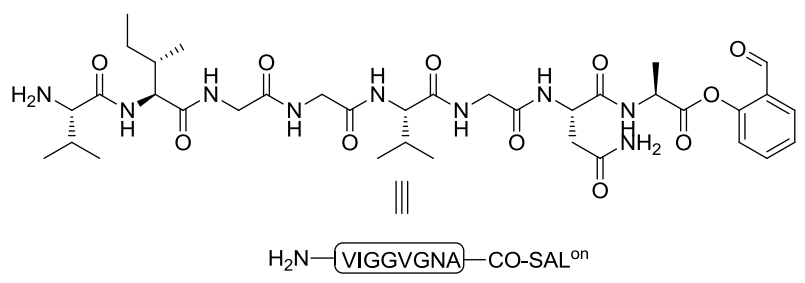

Crude protected peptide $B o c H N$-Val-Ile-Gly-Gly-Val-Gly-Asn(Trt)-COOH (prepared according to general experimental procedure A and B) (30.3 mg, $31.7 \mu \mathrm{mol})$ was dissolved in $\mathrm{CHCl}_{3} /$ trifluoroethanol $(2.0 \mathrm{~mL})$ and reacted with 
$\mathbf{H C l} \cdot \boldsymbol{H}_{2} \boldsymbol{N}$-Ala-CO-SAL off $(\mathbf{A})(27.2 \mathrm{mg}, 95.1 \mu \mathrm{mol})$ in the presence of EDC $(14.7 \mathrm{mg}$, $95.1 \mu \mathrm{mol})$ and HOOBt $(15.5 \mathrm{mg}, 95.1 \mu \mathrm{mol})$, as described in general procedure D. After stirring for $3 \mathrm{~h}$, the reaction mixture was concentrated and subjected to TFA/ $\mathrm{H}_{2} \mathrm{O}(95 / 5, v / v)$ and pyruvic acid $(223.3 \mu \mathrm{L}, 3.2 \mathrm{mmol})$ for $3 \mathrm{~h}$. Preparative HPLC purification (10-40\% $\mathrm{CH}_{3} \mathrm{CN} / \mathrm{H}_{2} \mathrm{O}$ over $30 \mathrm{~min}$ ) followed by concentration at reduced pressure and lyophilization afforded peptide $1 \mathbf{a}(22.0 \mathrm{mg}, 88 \%$ yield $)$ as a white powder.

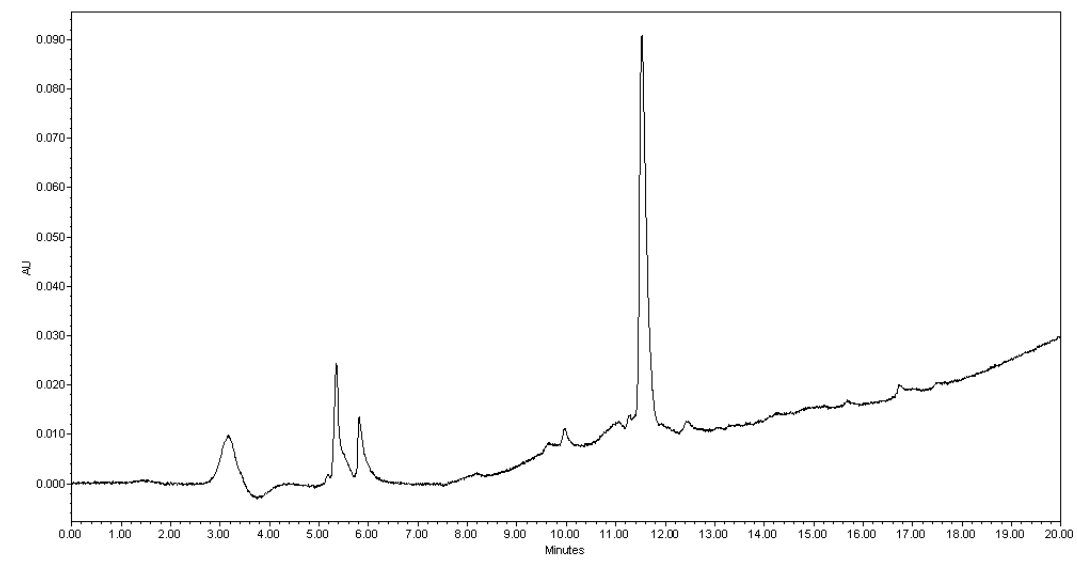

Figure S1. UV trace from analytical LC-MS analysis of purified peptide 1a: gradient $5-95 \% \mathrm{CH}_{3} \mathrm{CN} / \mathrm{H}_{2} \mathrm{O}$ containing $0.1 \% \mathrm{TFA}$ over $15 \mathrm{~min}$ at a flow rate of $0.6 \mathrm{~mL} / \mathrm{min}$.

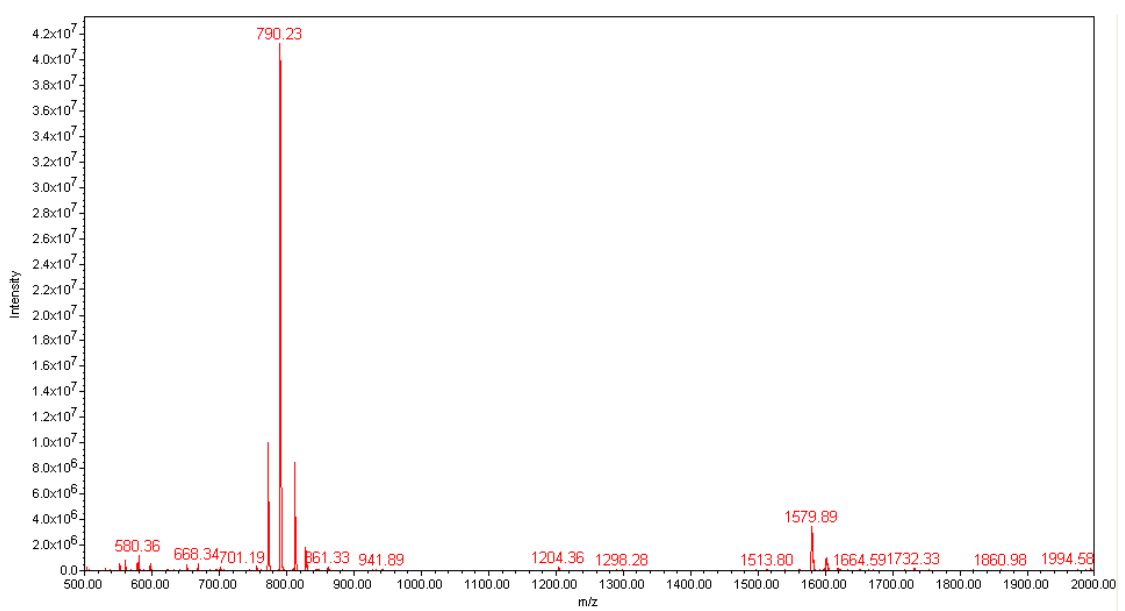

Figure S2. ESI-MS calcd. for $\mathrm{C}_{36} \mathrm{H}_{56} \mathrm{~N}_{9} \mathrm{O}_{11}[\mathrm{M}+\mathrm{H}]^{+} \mathrm{m} / z=790.41$, found 790.23; $[2 \mathrm{M}+\mathrm{H}]^{+} m / z=1579.81$, found 1579.89 .

Peptide 1b: $\mathrm{H}_{2} \mathrm{~N}$-VIGGVGNY-CO-SAL ${ }^{\text {on }}$ ester 


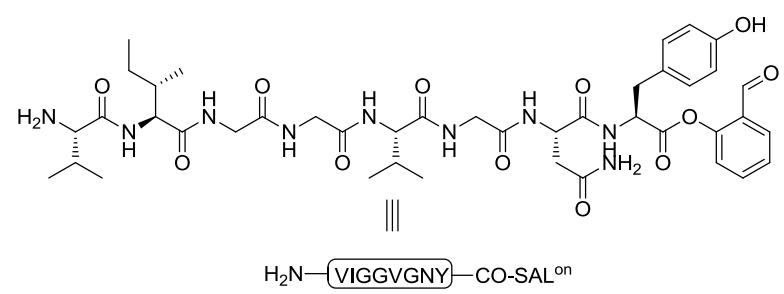

Crude protected peptide BocHN-Val-Ile-Gly-Gly-Val-Gly-Asn(Trt)-COOH (prepared according to general experimental procedure A and B) $(27.2 \mathrm{mg}, 28.5 \mu \mathrm{mol})$ was dissolved in $\mathrm{CHCl}_{3} /$ trifluoroethanol $(1.9 \mathrm{~mL})$ and reacted with $\mathbf{H C l} \cdot \boldsymbol{H}_{2} \boldsymbol{N}$-Tyr-CO-SAL ${ }^{\text {off }}(\mathbf{Y})(32.3 \mathrm{mg}, 85.4 \mu \mathrm{mol})$ in the presence of EDC (13.2 $\mathrm{mg}$, $85.4 \mu \mathrm{mol})$ and HOOBt $(13.9 \mathrm{mg}, 85.4 \mu \mathrm{mol})$, as described in general procedure D. After stirring for $3 \mathrm{~h}$, the reaction mixture was concentrated and subjected to TFA/ $\mathrm{H}_{2} \mathrm{O}(95 / 5, v / v)$ and pyruvic acid $(200.4 \mu \mathrm{L}, 2.9 \mathrm{mmol})$ for $3 \mathrm{~h}$. Preparative HPLC purification (10-40\% $\mathrm{CH}_{3} \mathrm{CN} / \mathrm{H}_{2} \mathrm{O}$ over $30 \mathrm{~min}$ ) followed by concentration at reduced pressure and lyophilization afforded peptide $\mathbf{1 b}(17.3 \mathrm{mg}, 69 \%$ yield) as a white powder.

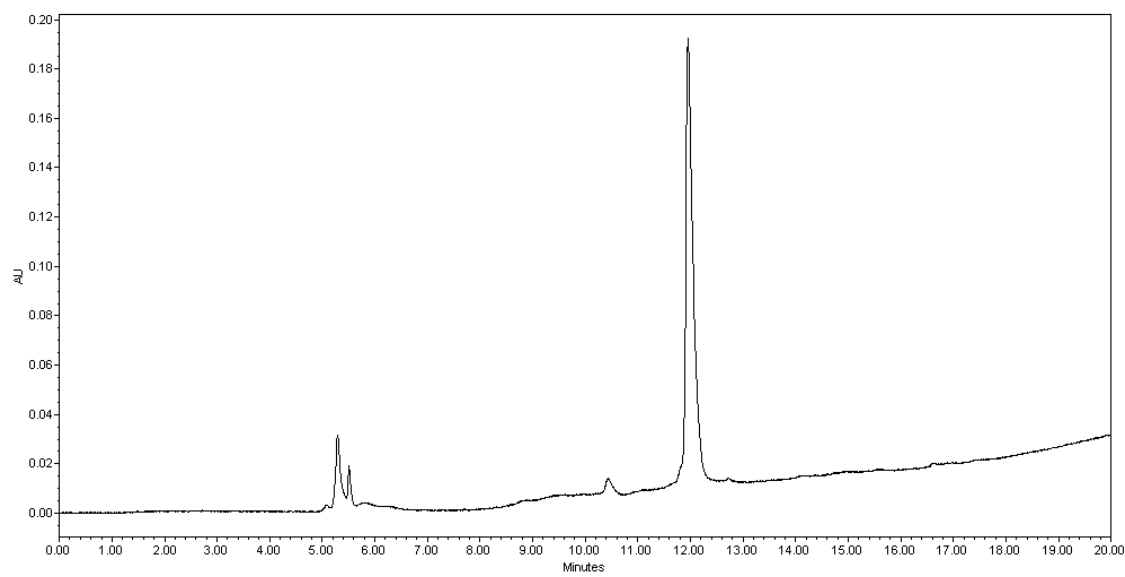

Figure S3. UV trace from analytical LC-MS analysis of purified peptide $\mathbf{1 b}$ : gradient $5-95 \% \mathrm{CH}_{3} \mathrm{CN} / \mathrm{H}_{2} \mathrm{O}$ containing $0.1 \% \mathrm{TFA}$ over $15 \mathrm{~min}$ at a flow rate of $0.6 \mathrm{~mL} / \mathrm{min}$. 


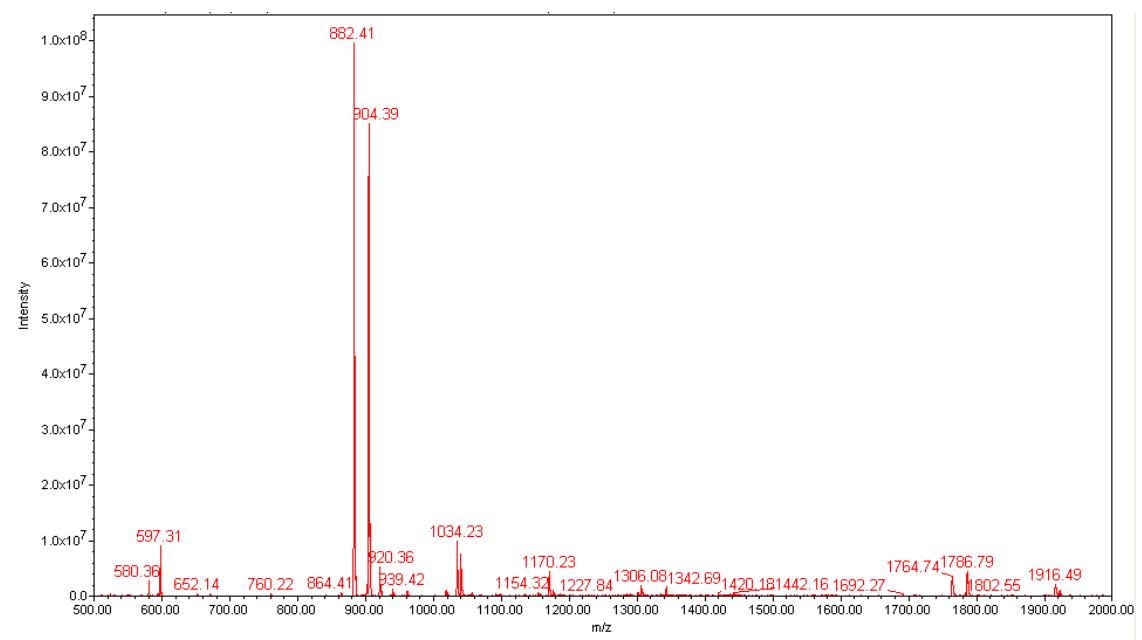

Figure S4. ESI-MS calcd. for $\mathrm{C}_{42} \mathrm{H}_{60} \mathrm{~N}_{9} \mathrm{O}_{12}[\mathrm{M}+\mathrm{H}]^{+} \mathrm{m} / z=882.44$, found 882.41; $[\mathrm{M}+\mathrm{Na}]^{+} m / z=904.42$, found 904.39 .

Peptide 1c: $\mathrm{H}_{2} \mathrm{~N}$-VIGGVGNV-CO-SAL ${ }^{\text {on }}$ ester

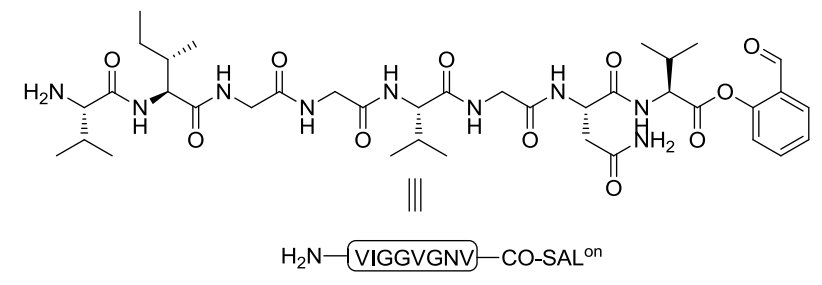

Crude protected peptide BocHN-Val-Ile-Gly-Gly-Val-Gly-Asn(Trt)-COOH (prepared according to general experimental procedure A and B) $(34.7 \mathrm{mg}, 36.3 \mu \mathrm{mol})$ was dissolved in $\mathrm{CHCl}_{3} /$ trifluoroethanol $(2.4 \mathrm{~mL})$ and reacted with $\mathbf{H C l} \cdot \boldsymbol{H}_{2} \boldsymbol{N}$-Val-CO-SAL ${ }^{\text {off }}(\mathbf{V})(34.2 \mathrm{mg}, 108.8 \mu \mathrm{mol})$ in the presence of EDC (16.9 $\mathrm{mg}, 108.8 \mu \mathrm{mol})$ and HOOBt $(17.7 \mathrm{mg}, 108.8 \mu \mathrm{mol})$, as described in general procedure D. After stirring for $3 \mathrm{~h}$, the reaction mixture was concentrated and subjected to $5.0 \mathrm{~mL}$ of $\mathrm{TFA} / \mathrm{H}_{2} \mathrm{O}(95 / 5, v / v)$ and pyruvic acid $(255.7 \mu \mathrm{L}, 3.6 \mathrm{mmol})$ for $3 \mathrm{~h}$. Preparative HPLC purification (10-40\% $\mathrm{CH}_{3} \mathrm{CN} / \mathrm{H}_{2} \mathrm{O}$ over $30 \mathrm{~min}$ ) followed by concentration at reduced pressure and lyophilization afforded peptide $1 \mathbf{c}$ ( $20.8 \mathrm{mg}$, $70 \%$ yield) as a white powder. 


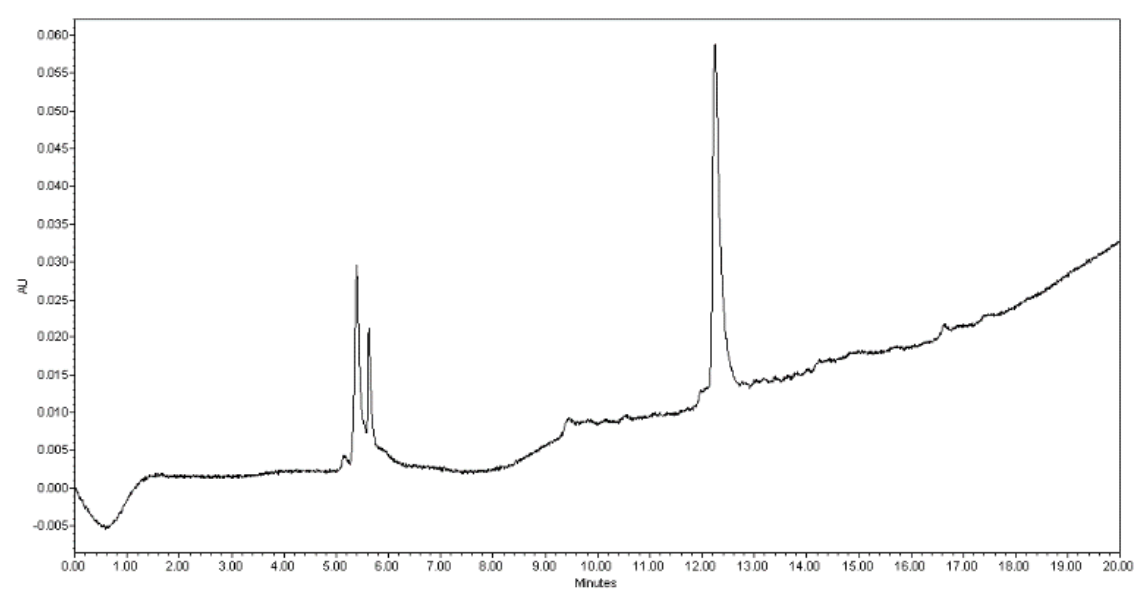

Figure S5. UV trace from analytical LC-MS analysis of purified peptide 1c: gradient $5-95 \% \mathrm{CH}_{3} \mathrm{CN} / \mathrm{H}_{2} \mathrm{O}$ containing $0.1 \%$ TFA over $15 \mathrm{~min}$ at a flow rate of $0.6 \mathrm{~mL} / \mathrm{min}$.

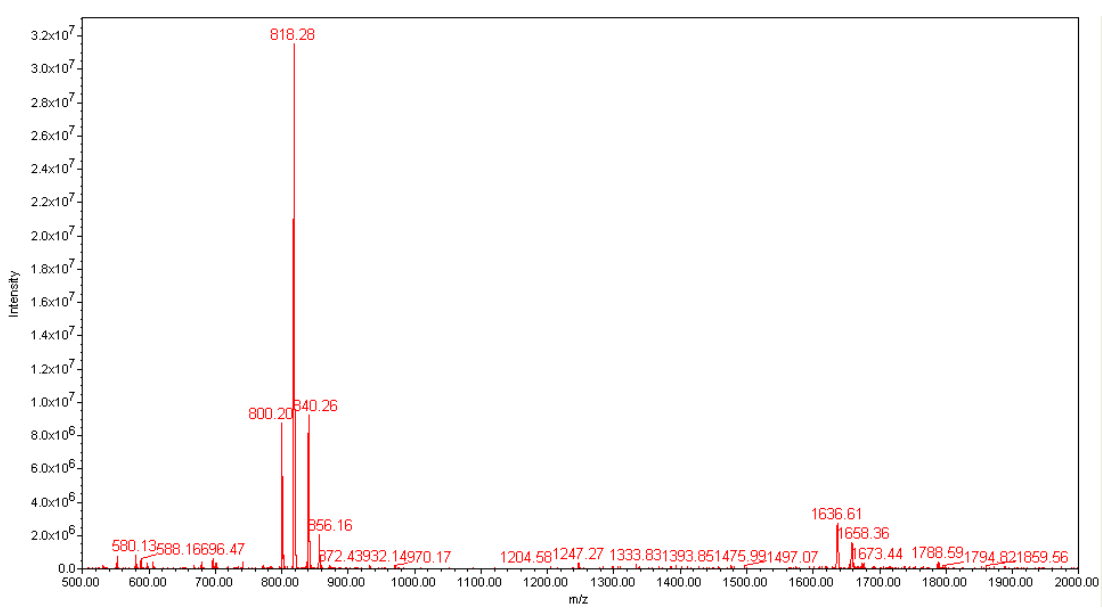

Figure S6. ESI-MS calcd. for $\mathrm{C}_{38} \mathrm{H}_{60} \mathrm{~N}_{9} \mathrm{O}_{11}[\mathrm{M}+\mathrm{H}]^{+} \mathrm{m} / z=818.44$, found 818.28; $[\mathrm{M}+\mathrm{Na}]^{+} \mathrm{m} / \mathrm{z}=840.42$, found $840.26 ;[2 \mathrm{M}+\mathrm{H}]^{+} \mathrm{m} / \mathrm{z}=1635.87$, found 1636.61; $[2 \mathrm{M}+\mathrm{Na}]^{+} \mathrm{m} / z=1657.86$, found 1658.36

\section{Peptide 1d: $\mathrm{H}_{2} \mathrm{~N}$-VIGGVGNS- $\mathrm{CO}$-SAL ${ }^{\text {on }}$ ester}

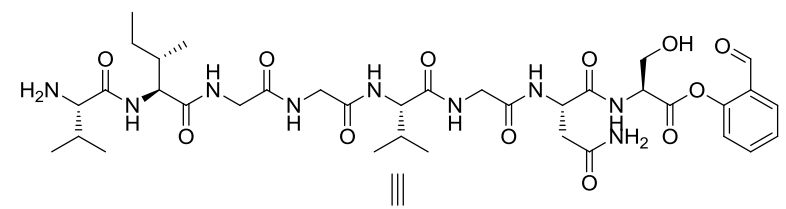

$\mathrm{H}_{2} \mathrm{~N}$-VIGGVGNS-CO-SAL on 
Crude protected peptide BocHN-Val-Ile-Gly-Gly-Val-Gly-Asn(Trt)-COOH (prepared according to general experimental procedure A and B) $(28.5 \mathrm{mg}, 29.8 \mu \mathrm{mol})$ was dissolved in $\mathrm{CHCl}_{3} /$ trifluoroethanol $(2.0 \mathrm{~mL})$ and reacted with $\mathbf{H C l} \cdot \boldsymbol{H}_{2} \boldsymbol{N}$-Ser-CO-SAL ${ }^{\text {off }}(\mathbf{S})(27 \mathrm{mg}, 89.4 \mu \mathrm{mol})$ in the presence of EDC (13.9 $\mathrm{mg}$, $89.4 \mu \mathrm{mol})$ and HOOBt $(14.6 \mathrm{mg}, 89.4 \mu \mathrm{mol})$, as described in general procedure D. After stirring for $3 \mathrm{~h}$, the reaction mixture was concentrated and subjected to $5.0 \mathrm{~mL}$ of TFA $/ \mathrm{H}_{2} \mathrm{O}(95 / 5, v / v)$ and pyruvic acid $(210.0 \mu \mathrm{L}, 3.0 \mathrm{mmol})$ for $3 \mathrm{~h}$. Preparative HPLC purification (10-40\% $\mathrm{CH}_{3} \mathrm{CN} / \mathrm{H}_{2} \mathrm{O}$ over $30 \mathrm{~min}$ ) followed by concentration at reduced pressure and lyophilization afforded peptide $1 \mathbf{1 d}(19.9 \mathrm{mg}, 83 \%$ yield) as a white powder.

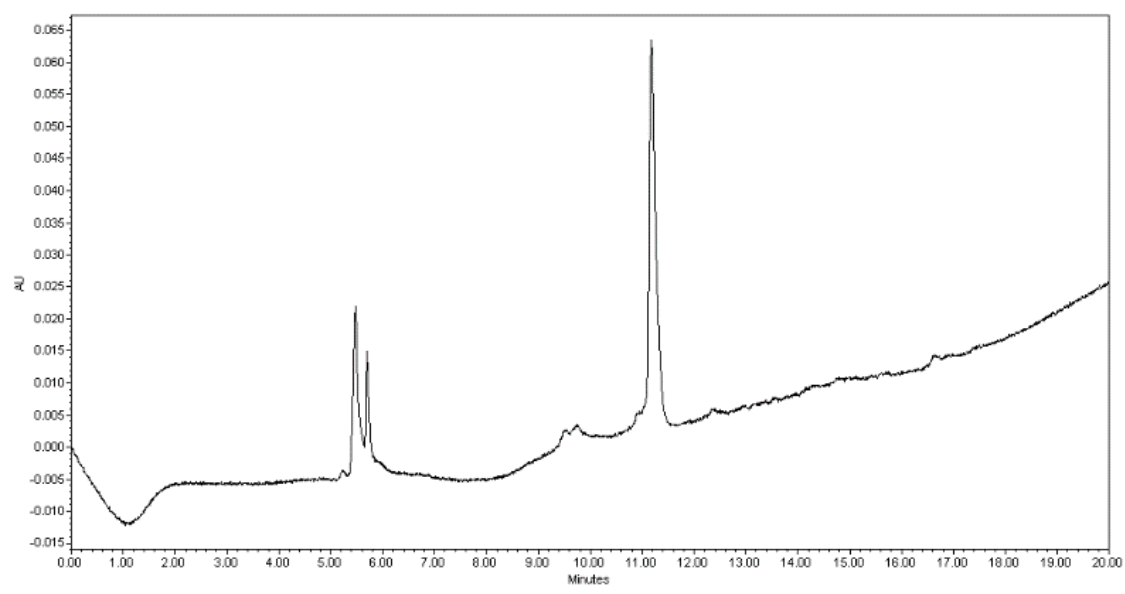

Figure S7. UV trace from analytical LC-MS analysis of purified peptide 1d: gradient $5-95 \% \mathrm{CH}_{3} \mathrm{CN} / \mathrm{H}_{2} \mathrm{O}$ containing $0.1 \%$ TFA over $15 \mathrm{~min}$ at a flow rate of $0.6 \mathrm{~mL} / \mathrm{min}$.

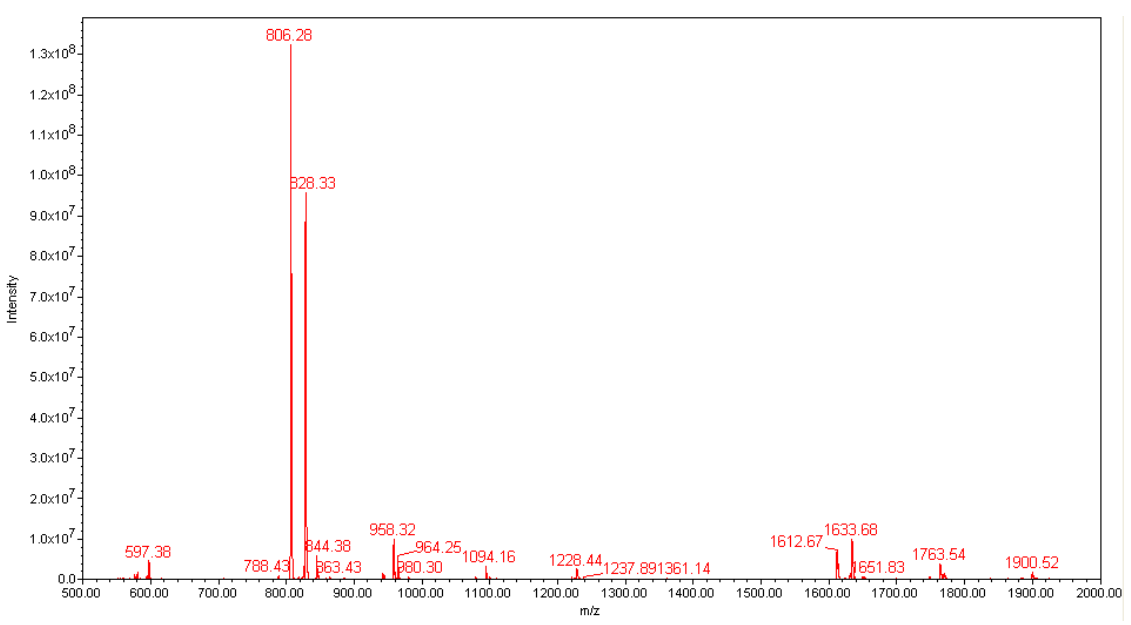


Figure S8. ESI-MS calcd. for $\mathrm{C}_{36} \mathrm{H}_{56} \mathrm{~N}_{9} \mathrm{O}_{12}[\mathrm{M}+\mathrm{H}]^{+} \mathrm{m} / \mathrm{z}=806.40$, found 806.28; $[\mathrm{M}+\mathrm{Na}]^{+} \mathrm{m} / \mathrm{z}=828.37$, found $828.33 ;[2 \mathrm{M}+\mathrm{H}]^{+} \mathrm{m} / \mathrm{z}=1611.80$, found 1612.67; $[2 \mathrm{M}+\mathrm{Na}]^{+} \mathrm{m} / z=1633.78$, found 1633.68 .

Peptide 1e: FmocHN-SEHDKTAY-CO-SAL ${ }^{\text {on }}$ ester

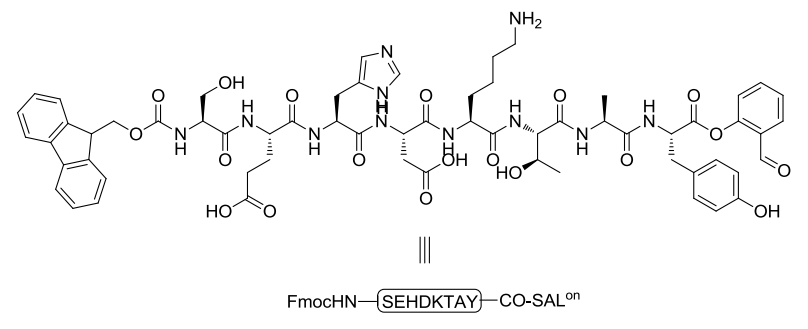

Crude

protected

peptide

FmocHN-Ser $\left({ }^{t} \mathrm{Bu}\right)-\mathrm{Glu}\left(\mathrm{O}^{t} \mathrm{Bu}\right)-\mathrm{His}(\mathrm{Trt})-\mathrm{Asp}\left(\mathrm{O}^{t} \mathrm{Bu}\right)-\mathrm{Lys}(\mathrm{Boc})-\mathrm{Thr}\left({ }^{t} \mathrm{Bu}\right)-\mathrm{Ala}-\mathrm{COOH}$

prepared according to general experimental procedure A and B) (248.0 mg, 157.5 $\mu \mathrm{mol})$ was dissolved in $\mathrm{CHCl}_{3} /$ trifluoroethanol $(10.5 \mathrm{~mL})$ and reacted with $\mathbf{H C l} \cdot \boldsymbol{H}_{2} \boldsymbol{N}$-Tyr- $\boldsymbol{C O}$-SAL ${ }^{\text {off }}(\mathbf{Y})(178.6 \mathrm{mg}, 472.4 \mu \mathrm{mol})$ in the presence of EDC (73.2 $\mathrm{mg}, 472.4 \mu \mathrm{mol})$ and HOOBt $(77.0 \mathrm{mg}, 472.4 \mu \mathrm{mol})$, as described in general procedure D. After stirring for $3 \mathrm{~h}$, the reaction mixture was concentrated and subjected to $7.0 \mathrm{~mL}$ of TFA/ $\mathrm{H}_{2} \mathrm{O}(95 / 5, v / v)$ and pyruvic acid $(1109.3 \mu \mathrm{L}, 15.7 \mathrm{mmol})$ for $3 \mathrm{~h}$. Preparative HPLC purification $\left(10-40 \% \mathrm{CH}_{3} \mathrm{CN} / \mathrm{H}_{2} \mathrm{O}\right.$ over $30 \mathrm{~min}$ ) followed by concentration at reduced pressure and lyophilization afforded peptide 1 e $(78.4 \mathrm{mg}$, $39 \%$ yield) as a white powder.

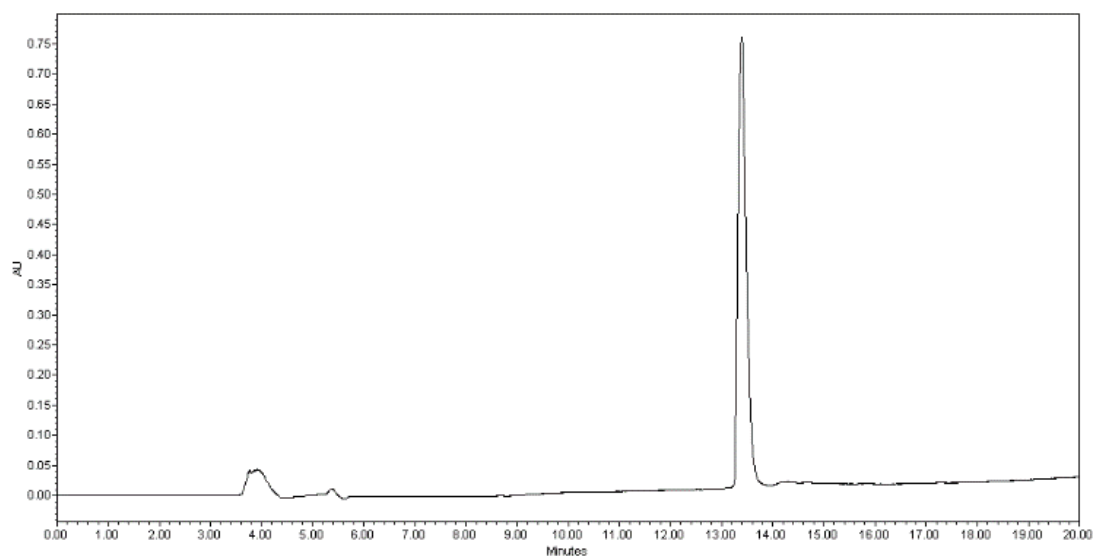


Figure S9. UV trace from analytical LC-MS analysis of purified peptide 1e: gradient $5-95 \% \mathrm{CH}_{3} \mathrm{CN} / \mathrm{H}_{2} \mathrm{O}$ containing $0.1 \% \mathrm{TFA}$ over $15 \mathrm{~min}$ at a flow rate of $0.6 \mathrm{~mL} / \mathrm{min}$.

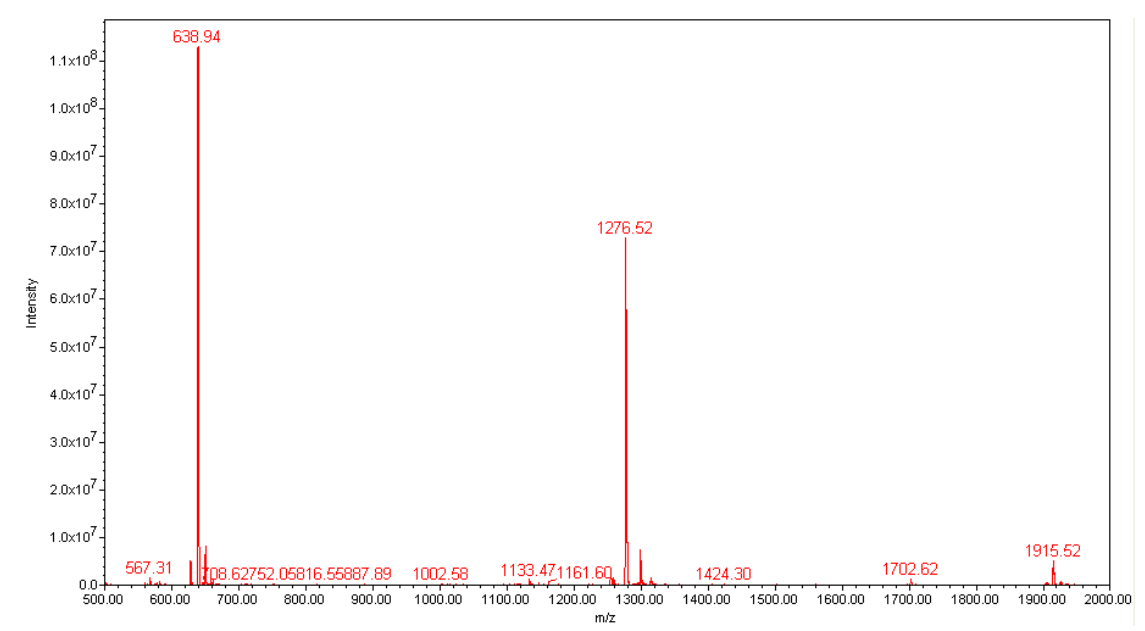

Figure S10. ESI-MS calcd. for $\mathrm{C}_{62} \mathrm{H}_{74} \mathrm{~N}_{11} \mathrm{O}_{19}[\mathrm{M}+\mathrm{H}]^{+} m / z=1276.52$, found 1276.52; $[\mathrm{M}+2 \mathrm{H}]^{2+} m / z=638.76$, found 638.94 ;

Peptide 1f: ZIGGVGNY-CO-SAL ${ }^{\text {on }}$ ester

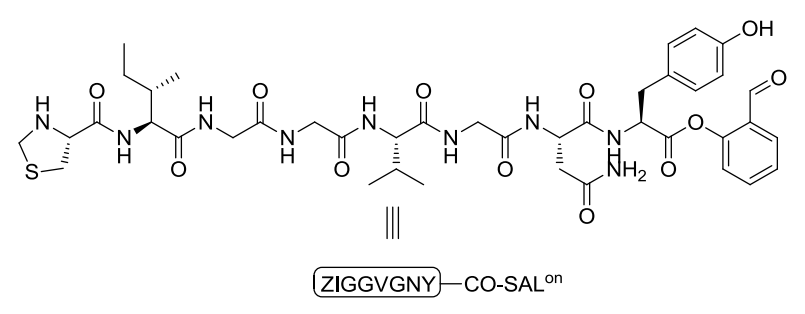

Crude protected peptide $B o c N-T h z-I l e-G l y-G l y-V a l-G l y-A s n(T r t)-C O O H$ (prepared according to general experimental procedure A and B) $(71.1 \mathrm{mg}, 73.1 \mu \mathrm{mol})$ was dissolved in $\mathrm{CHCl}_{3} /$ trifluoroethanol $(4.8 \mathrm{~mL})$ and reacted with $\mathbf{H C l} \cdot \boldsymbol{H}_{2} \boldsymbol{N}$-Tyr-CO-SAL ${ }^{\text {off }}(\mathbf{Y})(83.0 \mathrm{mg}, 219.4 \mu \mathrm{mol})$ in the presence of EDC (34.0 $\mathrm{mg}, 219.4 \mu \mathrm{mol})$ and HOOBt $(35.8 \mathrm{mg}, 219.4 \mu \mathrm{mol})$, as described in general procedure D. After stirring for $3 \mathrm{~h}$, the reaction mixture was concentrated and subjected to $5.0 \mathrm{~mL}$ of $\mathrm{TFA} / \mathrm{H}_{2} \mathrm{O}(95 / 5, v / v)$ and pyruvic acid $(515.3 \mu \mathrm{L}, 7.3 \mathrm{mmol})$ for $3 \mathrm{~h}$. Preparative HPLC purification $\left(10-40 \% \mathrm{CH}_{3} \mathrm{CN} / \mathrm{H}_{2} \mathrm{O}\right.$ over $30 \mathrm{~min}$ ) followed by concentration at reduced pressure and lyophilization afforded peptide $\mathbf{1 f}$ (16.4 $\mathrm{mg}$, $25 \%$ yield) as a white powder. 


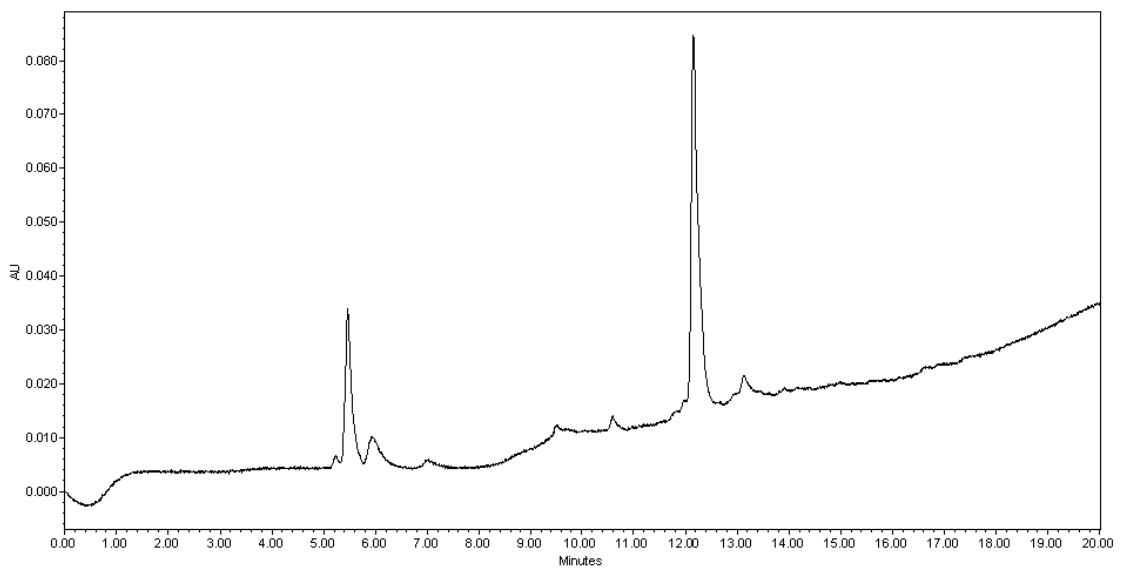

Figure S11. UV trace from analytical LC-MS analysis of purified peptide 1f: gradient $5-95 \% \mathrm{CH}_{3} \mathrm{CN} / \mathrm{H}_{2} \mathrm{O}$ containing $0.1 \%$ TFA over $15 \mathrm{~min}$ at a flow rate of $0.6 \mathrm{~mL} / \mathrm{min}$.

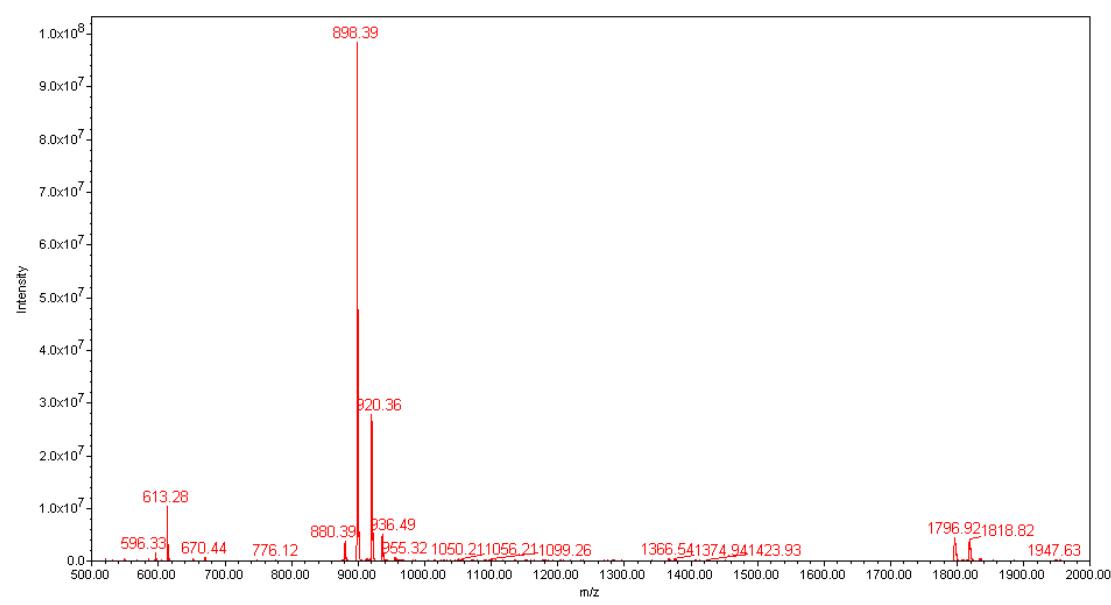

Figure S12. ESI-MS calcd. for $\mathrm{C}_{41} \mathrm{H}_{56} \mathrm{~N}_{9} \mathrm{O}_{12} \mathrm{~S}[\mathrm{M}+\mathrm{H}]^{+} m / z=898.38$, found 898.39; $[\mathrm{M}+\mathrm{Na}]^{+} \mathrm{m} / \mathrm{z}=920.37$, found $920.36 ;[2 \mathrm{M}+\mathrm{H}]^{+} \mathrm{m} / \mathrm{z}=1795.75$, found 1796.92; $[2 \mathrm{M}+\mathrm{Na}]^{+} \mathrm{m} / z=1817.73$, found 1818.82 .

Peptide 1g: $F m o c H N-S A R K L L Q D I M-C O-S A L{ }^{\text {on }}$ ester

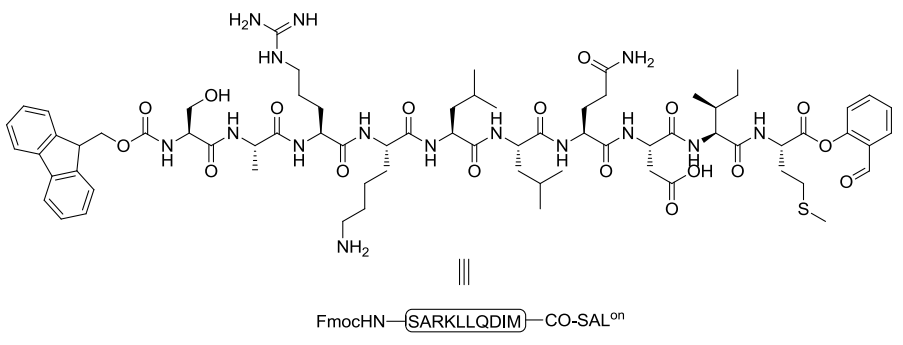


Crude

protected

peptide

FmocHN-Ser( $\left.{ }^{t} \mathrm{Bu}\right)-\mathrm{Ala}-\mathrm{Arg}(\mathrm{Pbf})-\mathrm{Lys}(\mathrm{Boc})-\mathrm{Leu}-\mathrm{Leu}-\mathrm{G} \ln (\mathrm{Trt})-\mathrm{Asp}\left(\mathrm{O}^{t} \mathrm{Bu}\right)-\mathrm{Ile}-\mathrm{OH}$

(prepared according to general experimental procedure A and B) (100.1 mg, 50.8 $\mu \mathrm{mol})$ was dissolved in $\mathrm{CHCl}_{3} /$ trifluoroethanol $(3.4 \mathrm{~mL})$ and reacted with $\mathbf{H C l} \cdot \boldsymbol{H}_{2} \boldsymbol{N}$-Met-CO-SAL ${ }^{\text {off }}(\mathbf{M})(52.7 \mathrm{mg}, 152.4 \mu \mathrm{mol})$ in the presence of EDC (23.6 $\mathrm{mg}, 152.4 \mu \mathrm{mol})$ and HOOBt $(24.8 \mathrm{mg}, 152.4 \mu \mathrm{mol})$, as described in general procedure D. After stirring for $3 \mathrm{~h}$, the reaction mixture was concentrated and subjected to $5.0 \mathrm{~mL}$ of TFA/ $\mathrm{H}_{2} \mathrm{O}(95 / 5, v / v)$ and pyruvic acid $(357.8 \mu \mathrm{L}, 5.1 \mathrm{mmol})$ for $3 \mathrm{~h}$. Preparative HPLC purification (10-40\% $\mathrm{CH}_{3} \mathrm{CN} / \mathrm{H}_{2} \mathrm{O}$ over $30 \mathrm{~min}$ ) followed by concentration at reduced pressure and lyophilization afforded peptide $\mathbf{1 g}$ (23.6 $\mathrm{mg}$, $31 \%$ yield) as a white powder.

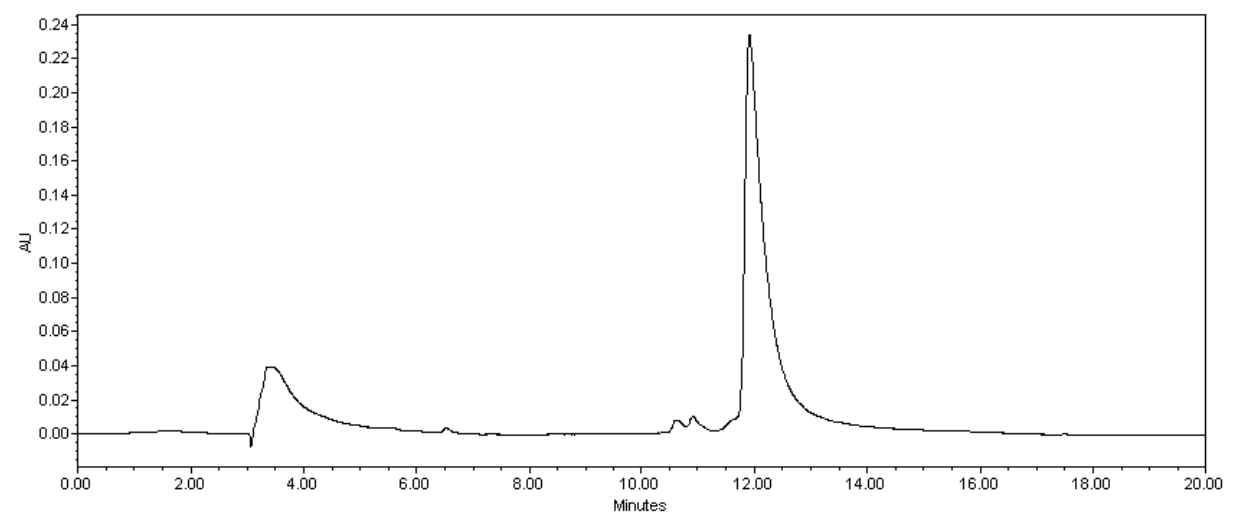

Figure S13. UV trace from analytical LC-MS analysis of purified peptide 1g: gradient 5-95\% $\mathrm{CH}_{3} \mathrm{CN} / \mathrm{H}_{2} \mathrm{O}$ containing $0.1 \%$ TFA over $15 \mathrm{~min}$ at a flow rate of 0.6 $\mathrm{mL} / \mathrm{min}$.

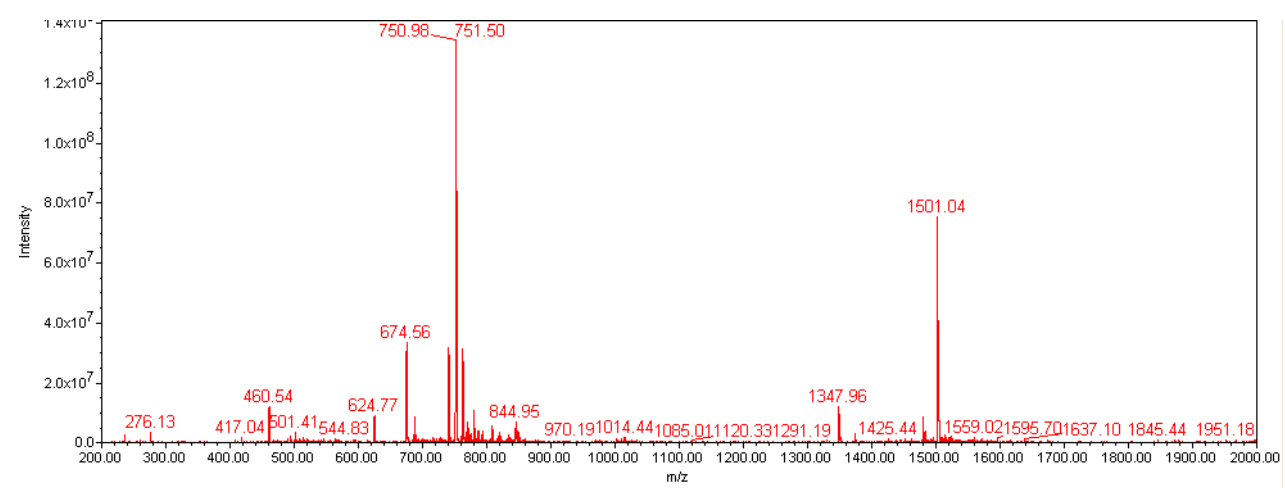

Figure S14. ESI-MS calcd. for $\mathrm{C}_{72} \mathrm{H}_{105} \mathrm{~N}_{15} \mathrm{O}_{18} \mathrm{~S}[\mathrm{M}+\mathrm{H}]^{+} \mathrm{m} / z=1500.75$, found 1501.04; $[\mathrm{M}+2 \mathrm{H}]^{2+} \mathrm{m} / \mathrm{z}=750.88$, found 750.98 .

Peptide 2a: $\mathrm{H}_{2} \mathrm{~N}$-TLHAPTDY-CO-SAL ${ }^{\text {off }}$ ester 


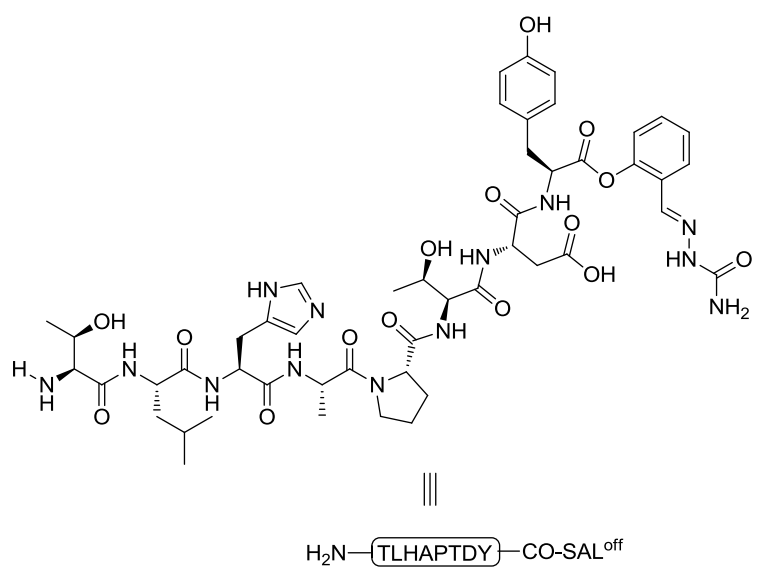

Crude

protected

peptide

BocHN-Thr $\left({ }^{t} \mathrm{Bu}\right)$-Leu-His(Trt)-Ala-Pro-Thr $\left({ }^{t} \mathrm{Bu}\right)-\mathrm{Asp}\left(\mathrm{O}^{t} \mathrm{Bu}\right)-\mathrm{COOH}$

(prepared according to general experimental procedure A and B) (32.9 mg, $26.0 \mu \mathrm{mol})$ was dissolved in $\mathrm{CHCl}_{3} /$ trifluoroethanol $(1.7 \mathrm{~mL})$ and reacted with $\mathbf{H C l} \cdot \boldsymbol{H}_{2} \boldsymbol{N}$-Tyr- $\boldsymbol{C O}$-SAL ${ }^{\text {off }}(\mathbf{Y})(29.5 \mathrm{mg}, 78.1 \mu \mathrm{mol})$ in the presence of EDC $(12.1 \mathrm{mg}$, $78.1 \mu \mathrm{mol})$ and HOOBt $(12.7 \mathrm{mg}, 78.1 \mu \mathrm{mol})$, as described in general procedure D. After stirring for $3 \mathrm{~h}$, the reaction mixture was concentrated and subjected to $5.0 \mathrm{~mL}$ of TFA $/ \mathrm{H}_{2} \mathrm{O}(95 / 5, v / v)$ for $1 \mathrm{~h}$. Preparative HPLC purification $\left(15-35 \% \mathrm{CH}_{3} \mathrm{CN} / \mathrm{H}_{2} \mathrm{O}\right.$ over $30 \mathrm{~min}$ ) followed by concentration at reduced pressure and lyophilization afforded peptide $\mathbf{2 a}(17.1 \mathrm{mg}, 61 \%$ yield $)$ as a white powder.

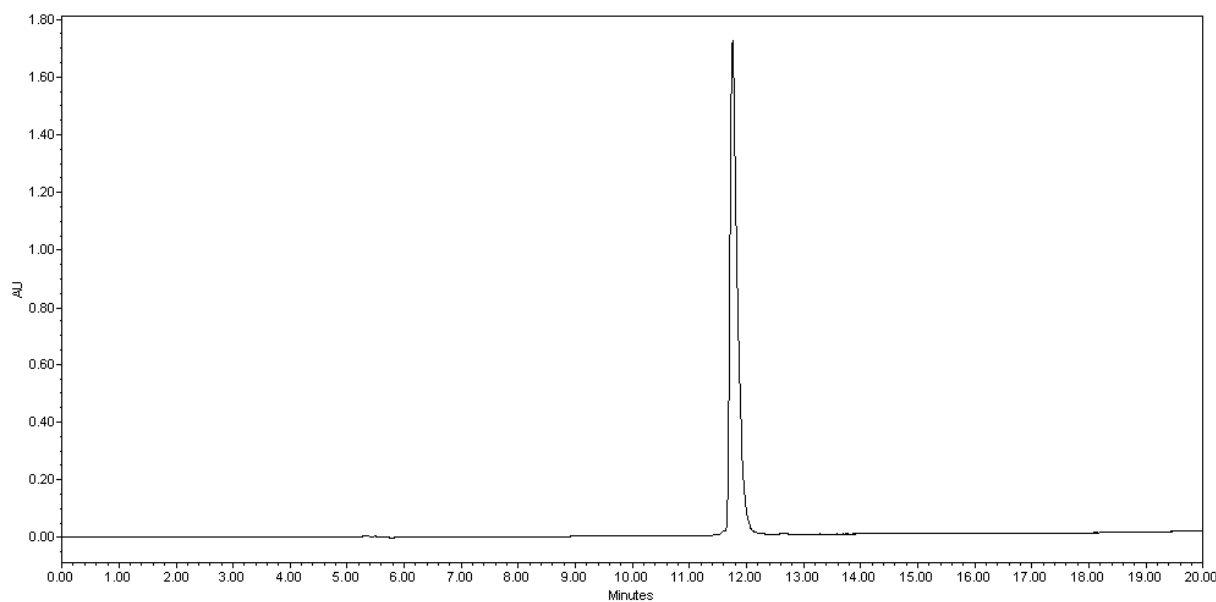

Figure S15. UV trace from analytical LC-MS analysis of purified peptide 2a: gradient 5-95\% $\mathrm{CH}_{3} \mathrm{CN} / \mathrm{H}_{2} \mathrm{O}$ containing $0.1 \%$ TFA over $15 \mathrm{~min}$ at a flow rate of 0.6 $\mathrm{mL} / \mathrm{min}$. 


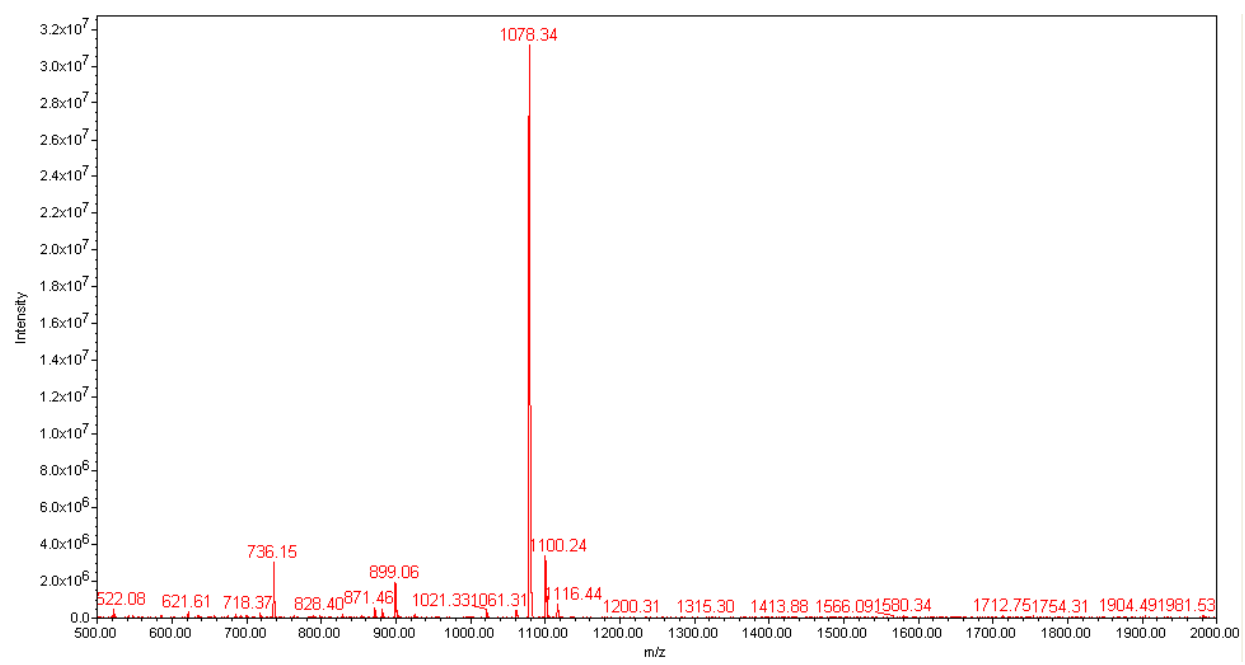

Figure S16. ESI-MS calcd. for $\mathrm{C}_{49} \mathrm{H}_{68} \mathrm{~N}_{13} \mathrm{O}_{15}[\mathrm{M}+\mathrm{H}]^{+} \mathrm{m} / z=1078.50$, found 1078.34; $[\mathrm{M}+\mathrm{Na}]^{+} m / z=1100.47$, found 1100.24 .

Peptide 2b: $\mathrm{H}_{2} \mathrm{~N}$-TLHAPTDS-CO-SAL ${ }^{\text {off }}$ ester

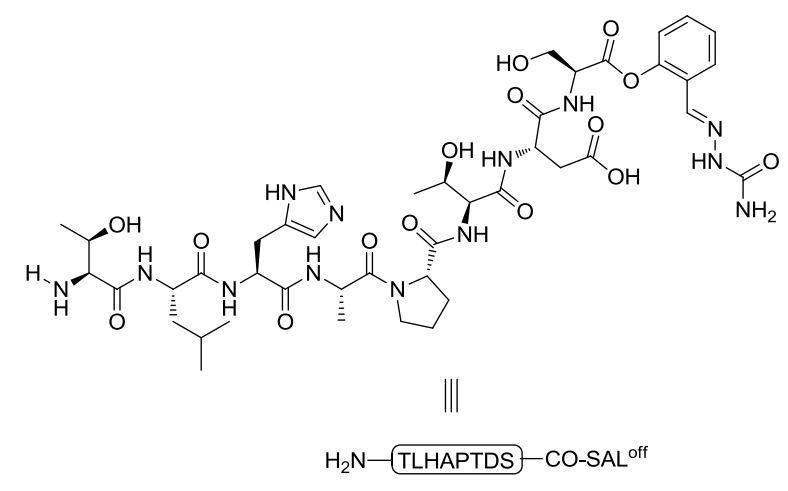

Crude

protected

peptide

$B o c H N-\operatorname{Thr}\left({ }^{t} \mathrm{Bu}\right)-$ Leu-His(Trt)-Ala-Pro-Thr $\left({ }^{t} \mathrm{Bu}\right)-\mathrm{Asp}\left(\mathrm{O}^{t} \mathrm{Bu}\right)-\mathrm{COOH}$

(prepared according to general experimental procedure A and B) $(31.1 \mathrm{mg}, 24.6 \mu \mathrm{mol})$ was dissolved in $\mathrm{CHCl}_{3} /$ trifluoroethanol $(1.6 \mathrm{~mL})$ and reacted with $\mathbf{H C l} \cdot \boldsymbol{H}_{2} \boldsymbol{N}$-Ser- $\boldsymbol{C O}$-SAL ${ }^{\text {off }} \mathbf{( S )}(22.3 \mathrm{mg}, 73.8 \mu \mathrm{mol})$ in the presence of EDC (11.4 $\mathrm{mg}$, $73.8 \mu \mathrm{mol})$ and HOOBt $(12.0 \mathrm{mg}, 73.8 \mu \mathrm{mol})$, as described in general procedure D. After stirring for $3 \mathrm{~h}$, the reaction mixture was concentrated and subjected to $5.0 \mathrm{~mL}$ of TFA/ $\mathrm{H}_{2} \mathrm{O}(95 / 5, v / v)$ for $1 \mathrm{~h}$. Preparative HPLC purification $\left(15-35 \% \mathrm{CH}_{3} \mathrm{CN} / \mathrm{H}_{2} \mathrm{O}\right.$ over $30 \mathrm{~min}$ ) followed by concentration at reduced pressure and lyophilization afforded compound $\mathbf{2 b}$ (17.8 $\mathrm{mg}, 72 \%$ yield) as a white powder. 


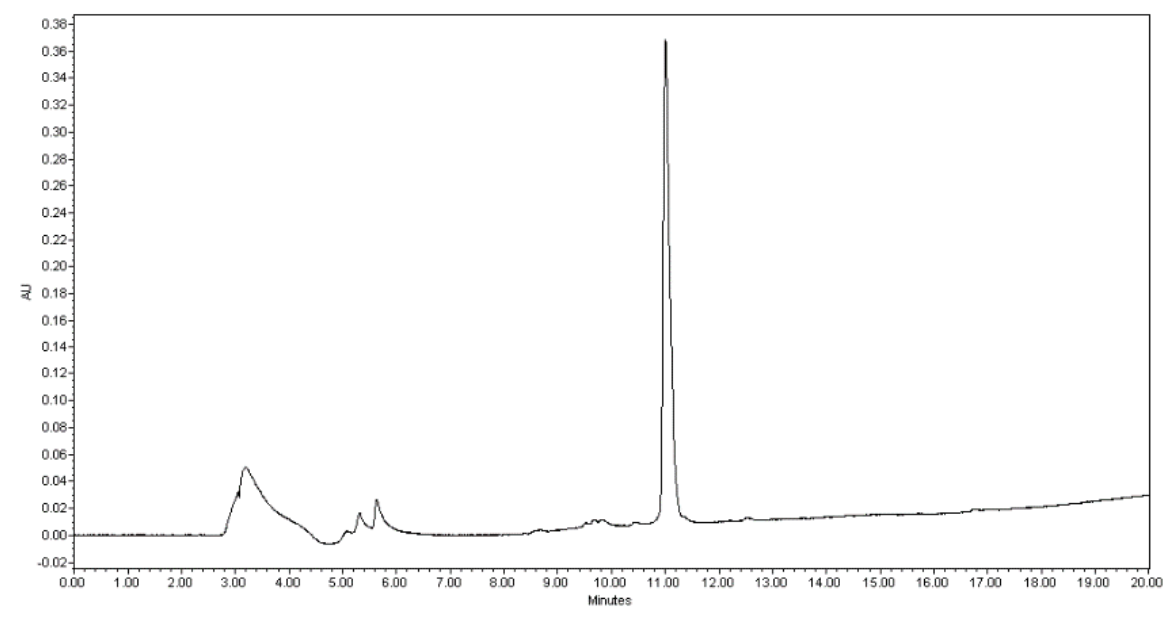

Figure S17. UV trace from analytical LC-MS analysis of purified peptide $\mathbf{2 b}$ :

gradient 5-95\% $\mathrm{CH}_{3} \mathrm{CN} / \mathrm{H}_{2} \mathrm{O}$ containing $0.1 \%$ TFA over $15 \mathrm{~min}$ at a flow rate of 0.6 $\mathrm{mL} / \mathrm{min}$.

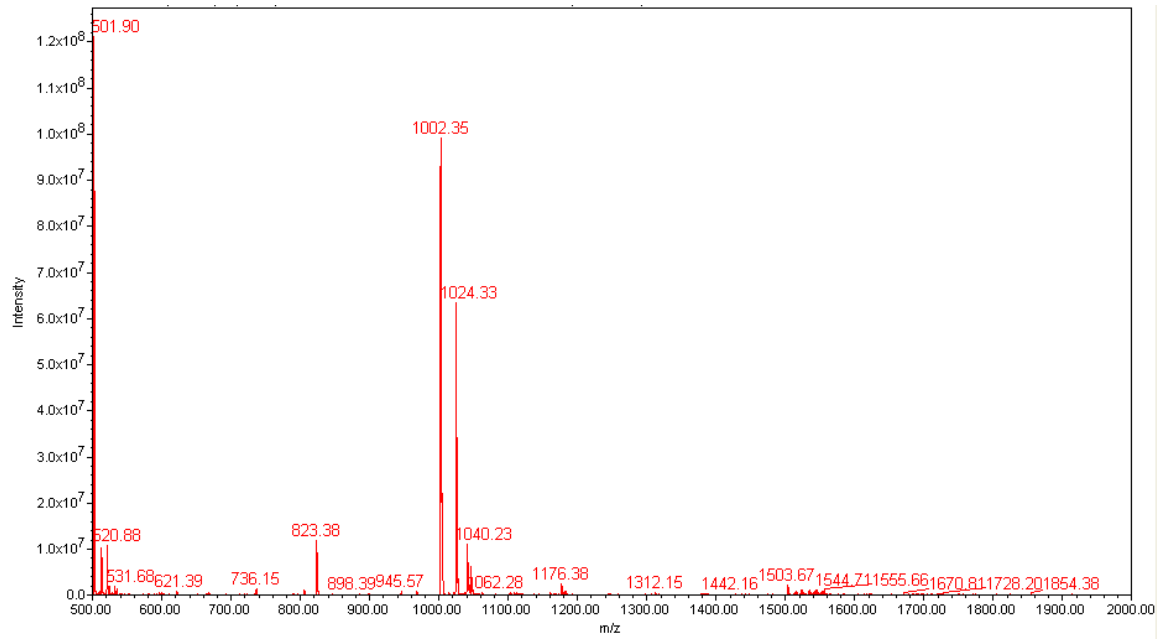

Figure S18. ESI-MS calcd. for $\mathrm{C}_{43} \mathrm{H}_{64} \mathrm{~N}_{13} \mathrm{O}_{15}[\mathrm{M}+\mathrm{H}]^{+} m / z=1002.46$, found 1002.35; $[\mathrm{M}+\mathrm{Na}]^{+} \mathrm{m} / \mathrm{z}=1024.45$, found 1024.33; $[\mathrm{M}+2 \mathrm{H}]^{2+} \mathrm{m} / \mathrm{z}=501.74$, found 501.90 .

Peptide 2c: $\mathrm{H}_{2} \mathrm{~N}$-TLHAPTDA-CO-SAL ${ }^{\text {off }}$ ester 


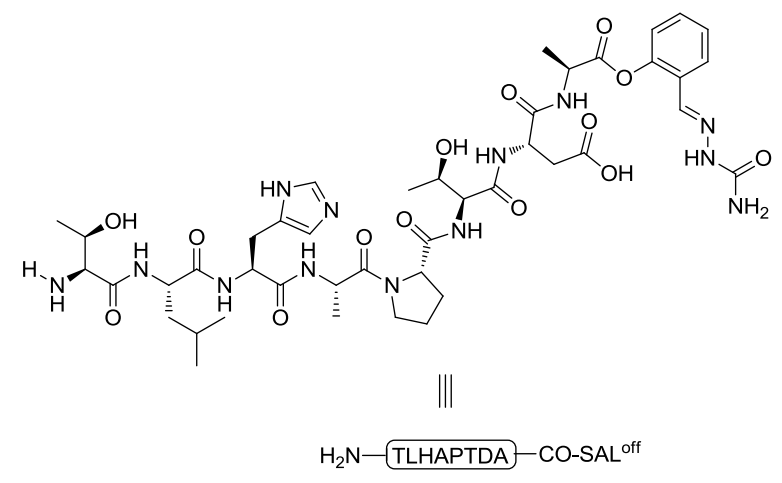

Crude

protected

peptide

BocHN-Thr $\left({ }^{t} \mathrm{Bu}\right)$-Leu-His(Trt)-Ala-Pro-Thr $\left({ }^{t} \mathrm{Bu}\right)-\mathrm{Asp}\left(\mathrm{O}^{t} \mathrm{Bu}\right)-\mathrm{COOH}$

(prepared according to general experimental procedure A and B) $(30.8 \mathrm{mg}, 24.4 \mu \mathrm{mol})$ was dissolved in $\mathrm{CHCl}_{3} /$ trifluoroethanol $(1.6 \mathrm{~mL})$ and reacted with $\mathbf{H C l} \cdot \boldsymbol{H}_{2} \boldsymbol{N}$-Ala-CO-SAL ${ }^{\text {off }} \mathbf{( A )}(20.9 \mathrm{mg}, 73.1 \mu \mathrm{mol})$ in the presence of EDC (11.3 $\mathrm{mg}$, $73.1 \mu \mathrm{mol})$ and HOOBt $(11.9 \mathrm{mg}, 73.1 \mu \mathrm{mol})$, as described in general procedure D. After stirring for $3 \mathrm{~h}$, the reaction mixture was concentrated and subjected to $5.0 \mathrm{~mL}$ of TFA/ $\mathrm{H}_{2} \mathrm{O}(95 / 5, v / v)$ for $1 \mathrm{~h}$. Preparative HPLC purification $\left(15-35 \% \mathrm{CH}_{3} \mathrm{CN} / \mathrm{H}_{2} \mathrm{O}\right.$ over $30 \mathrm{~min}$ ) followed by concentration at reduced pressure and lyophilization afforded compound $2 \mathrm{c}$ (15.9 $\mathrm{mg}, 66 \%$ yield) as a white powder.

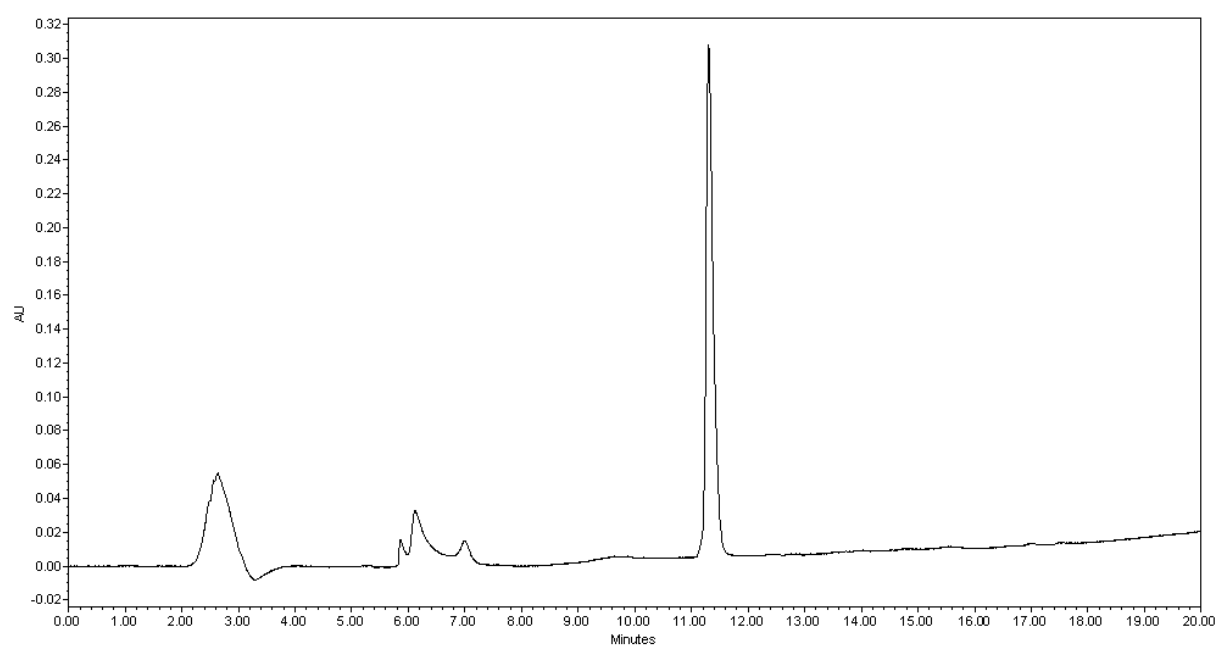

Figure S19. UV trace from analytical LC-MS analysis of purified peptide 2c: gradient $5-95 \% \mathrm{CH}_{3} \mathrm{CN} / \mathrm{H}_{2} \mathrm{O}$ containing $0.1 \%$ TFA over $15 \mathrm{~min}$ at a flow rate of $0.6 \mathrm{~mL} / \mathrm{min}$. 


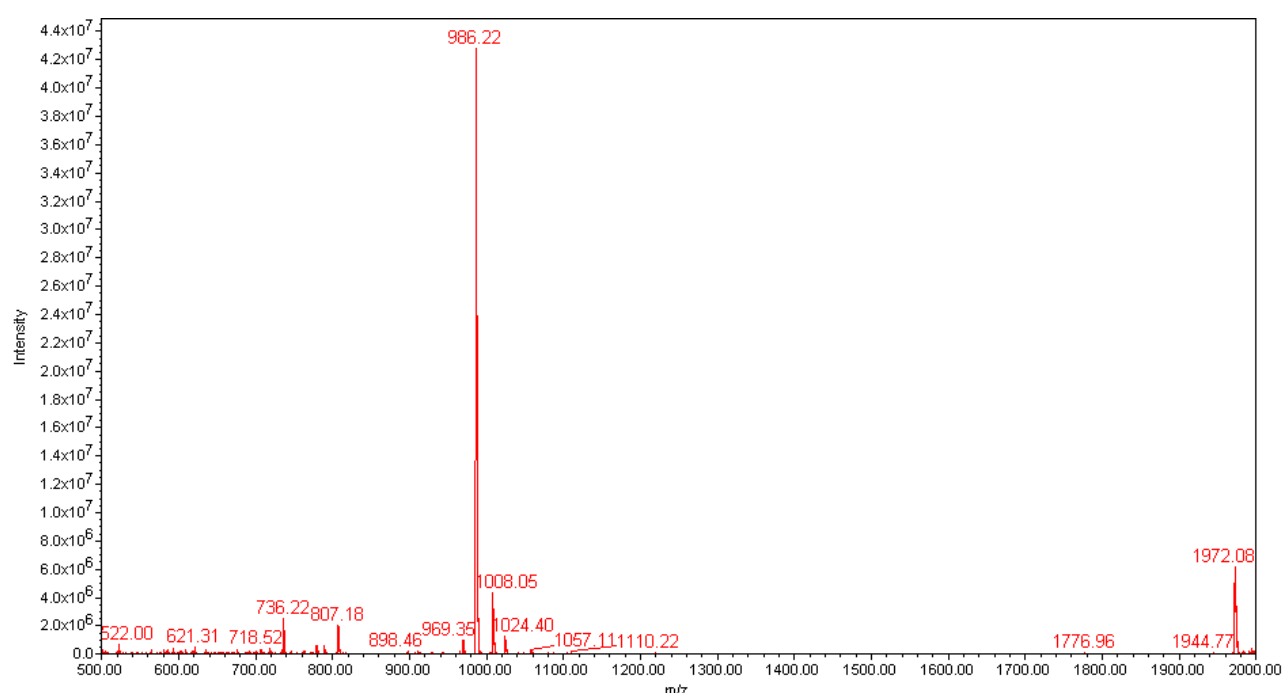

Figure S20. ESI-MS calcd. for $\mathrm{C}_{43} \mathrm{H}_{64} \mathrm{~N}_{13} \mathrm{O}_{14}[\mathrm{M}+\mathrm{H}]^{+} m / z=986.47$, found 986.22; $[\mathrm{M}+\mathrm{Na}]^{+} m / z=1008.45$, found 1008.05; $[2 \mathrm{M}+\mathrm{H}]^{+} \mathrm{m} / z=1971.93$, found 1972.08.

\section{Peptide 2d: $\mathrm{H}_{2} \mathrm{~N}$-TLHAPTDV-CO-SAL ${ }^{\text {off }}$ ester}

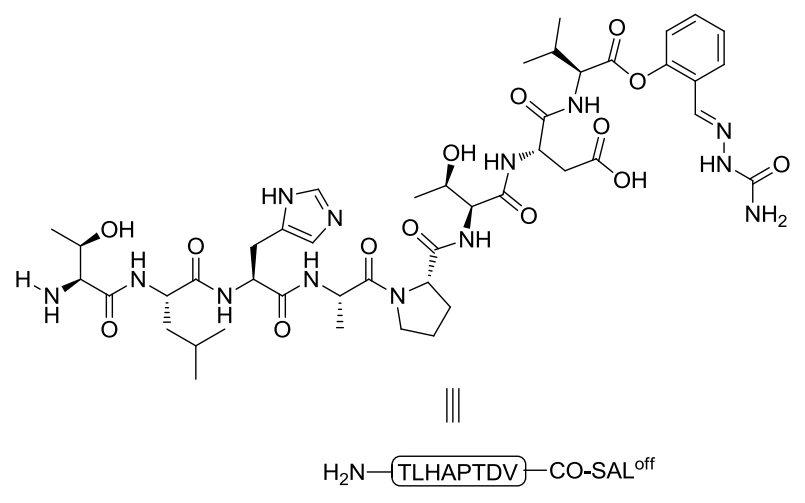

Crude

protected

peptide

BocHN-Thr $\left({ }^{t} \mathrm{Bu}\right)$-Leu-His(Trt)-Ala-Pro-Thr $\left({ }^{t} \mathrm{Bu}\right)-\mathrm{Asp}\left(\mathrm{O}^{t} \mathrm{Bu}\right)-\mathrm{COOH}$

(prepared according to general experimental procedure A and B) $(32.3 \mathrm{mg}, 25.6 \mu \mathrm{mol})$ was dissolved in $\mathrm{CHCl}_{3} /$ trifluoroethanol $(1.7 \mathrm{~mL})$ and reacted with $\mathbf{H C l} \cdot \mathbf{H}_{2} \boldsymbol{N}$-Val-CO-SAL ${ }^{\text {off }}(\mathbf{V})(24.1 \mathrm{mg}, 76.7 \mu \mathrm{mol})$ in the presence of EDC $(11.9 \mathrm{mg}$, $76.7 \mu \mathrm{mol})$ and HOOBt $(12.5 \mathrm{mg}, 76.7 \mu \mathrm{mol})$, as described in general procedure D. After stirring for $3 \mathrm{~h}$, the reaction mixture was concentrated and subjected to $5.0 \mathrm{~mL}$ of TFA/ $\mathrm{H}_{2} \mathrm{O}(95 / 5, v / v)$ for $1 \mathrm{~h}$. Preparative HPLC purification $\left(15-35 \% \mathrm{CH}_{3} \mathrm{CN} / \mathrm{H}_{2} \mathrm{O}\right.$ over $30 \mathrm{~min}$ ) followed by concentration at reduced pressure and lyophilization afforded compound $\mathbf{2 d}$ (17.9 $\mathrm{mg}, 69 \%$ yield) as a white powder. 


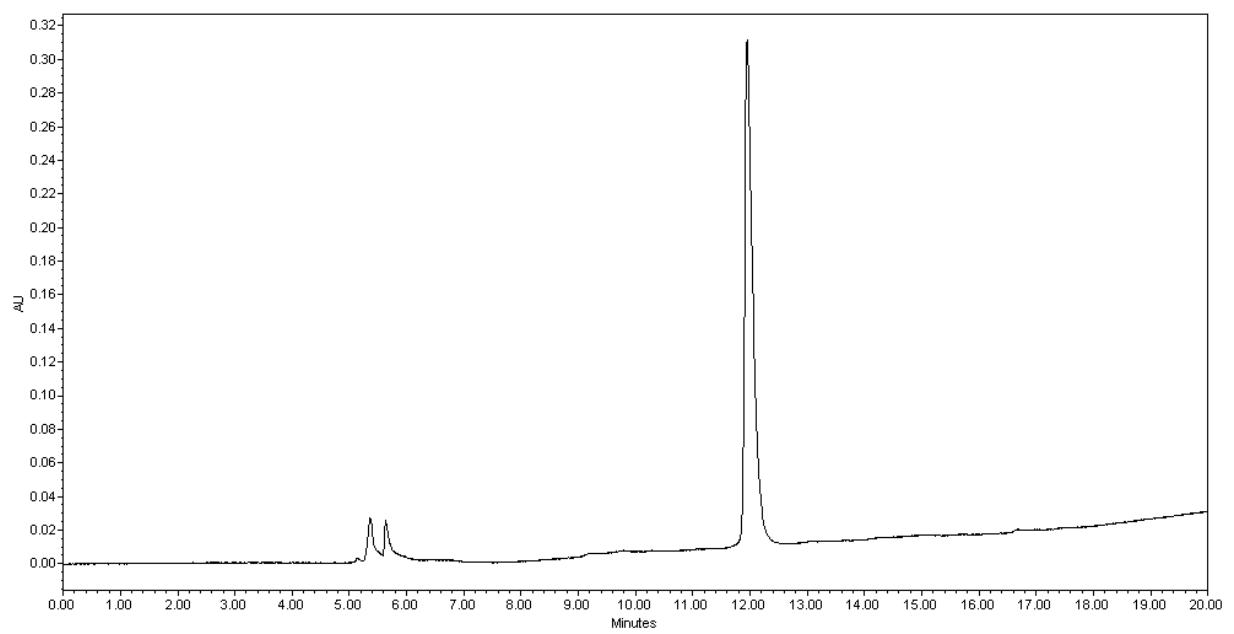

Figure S21. UV trace from analytical LC-MS analysis of purified peptide 2d:

gradient 5-95\% $\mathrm{CH}_{3} \mathrm{CN} / \mathrm{H}_{2} \mathrm{O}$ containing $0.1 \%$ TFA over $15 \mathrm{~min}$ at a flow rate of 0.6 $\mathrm{mL} / \mathrm{min}$.

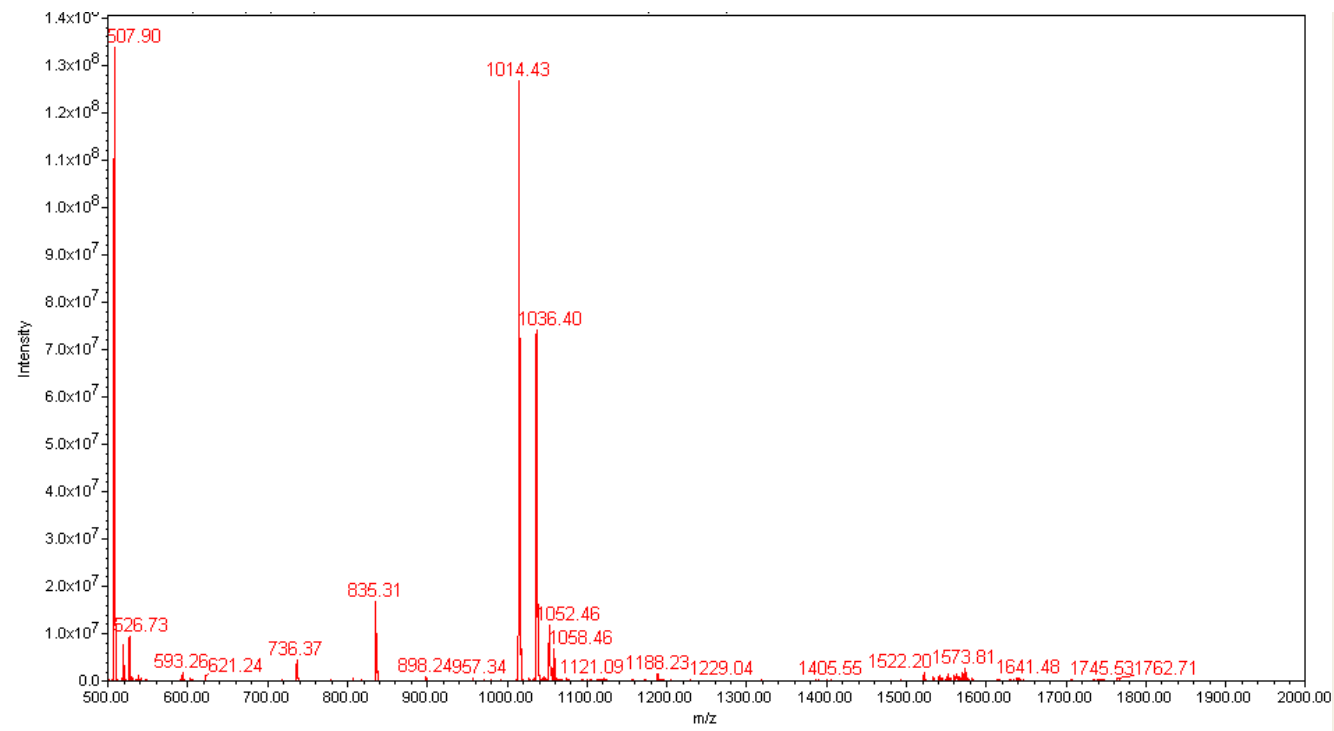

Figure S22. ESI-MS calcd. for $\mathrm{C}_{45} \mathrm{H}_{68} \mathrm{~N}_{13} \mathrm{O}_{14}[\mathrm{M}+\mathrm{H}]^{+} \mathrm{m} / z=1014.50$, found 1014.43; $[\mathrm{M}+\mathrm{Na}]^{+} \mathrm{m} / \mathrm{z}=1036.48$, found $1036.40 ;[\mathrm{M}+2 \mathrm{H}]^{2+} \mathrm{m} / \mathrm{z}=507.75$, found 507.90 .

Peptide 2e: $\mathrm{H}_{2} \mathrm{~N}$-TNSYRKVLGQL-CO-SAL ${ }^{\text {off }}$ ester 


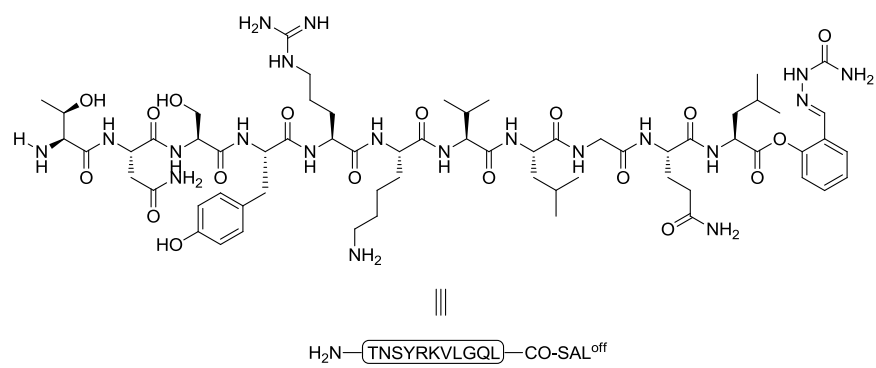

Crude

protected

peptide

$B o c H N-\operatorname{Thr}\left({ }^{t} \mathrm{Bu}\right)-\mathrm{Asn}(\mathrm{Trt})-\mathrm{Ser}\left({ }^{t} \mathrm{Bu}\right)-\mathrm{Tyr}\left({ }^{t} \mathrm{Bu}\right)-\operatorname{Arg}(\mathrm{Pbf})-\mathrm{Lys}(\mathrm{Boc})-\mathrm{Val}-\mathrm{Leu}-\mathrm{Gly}-\mathrm{Gln}(\mathrm{T}$

$\mathrm{rt})-\mathrm{COOH}$ (prepared according to general experimental procedure A and B) $(50.0 \mathrm{mg}$, $22.6 \mu \mathrm{mol})$ was dissolved in $\mathrm{CHCl}_{3} /$ trifluoroethanol $(1.5 \mathrm{~mL})$ and reacted with $\mathbf{H C l} \cdot \boldsymbol{H}_{2} \boldsymbol{N}$-Leu-CO-SAL ${ }^{\text {off }}(\mathbf{L})(22.2 \mathrm{mg}, 67.8 \mu \mathrm{mol})$ in the presence of EDC $(10.5 \mathrm{mg}$, $67.8 \mu \mathrm{mol})$ and HOOBt $(11.0 \mathrm{mg}, 67.8 \mu \mathrm{mol})$, as described in general procedure D. After stirring for $3 \mathrm{~h}$, the reaction mixture was concentrated and subjected to $5.0 \mathrm{~mL}$ of TFA $/ \mathrm{H}_{2} \mathrm{O}(95 / 5, v / v)$ for $1 \mathrm{~h}$. Preparative HPLC purification $\left(15-35 \% \mathrm{CH}_{3} \mathrm{CN} / \mathrm{H}_{2} \mathrm{O}\right.$ over $30 \mathrm{~min}$ ) followed by concentration at reduced pressure and lyophilization afforded peptide $\mathbf{2 e}$ (15.9 $\mathrm{mg}, 49 \%$ yield) as a white powder.

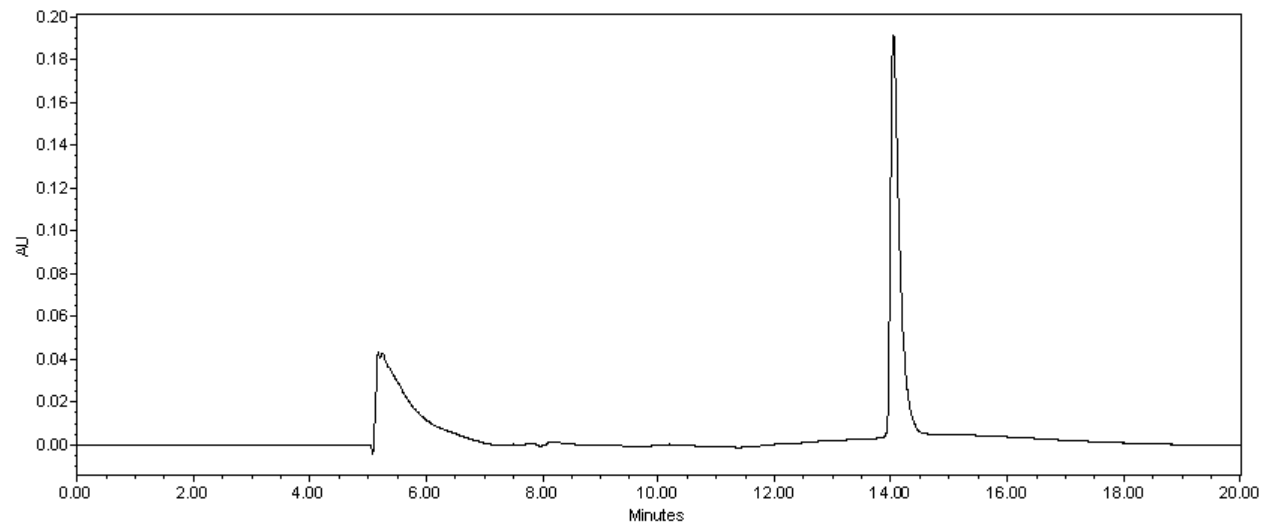

Figure S23. UV trace from analytical LC-MS analysis of purified peptide 2e: gradient $5-95 \% \mathrm{CH}_{3} \mathrm{CN} / \mathrm{H}_{2} \mathrm{O}$ containing $0.1 \%$ TFA over $15 \mathrm{~min}$ at a flow rate of $0.6 \mathrm{~mL} / \mathrm{min}$. 


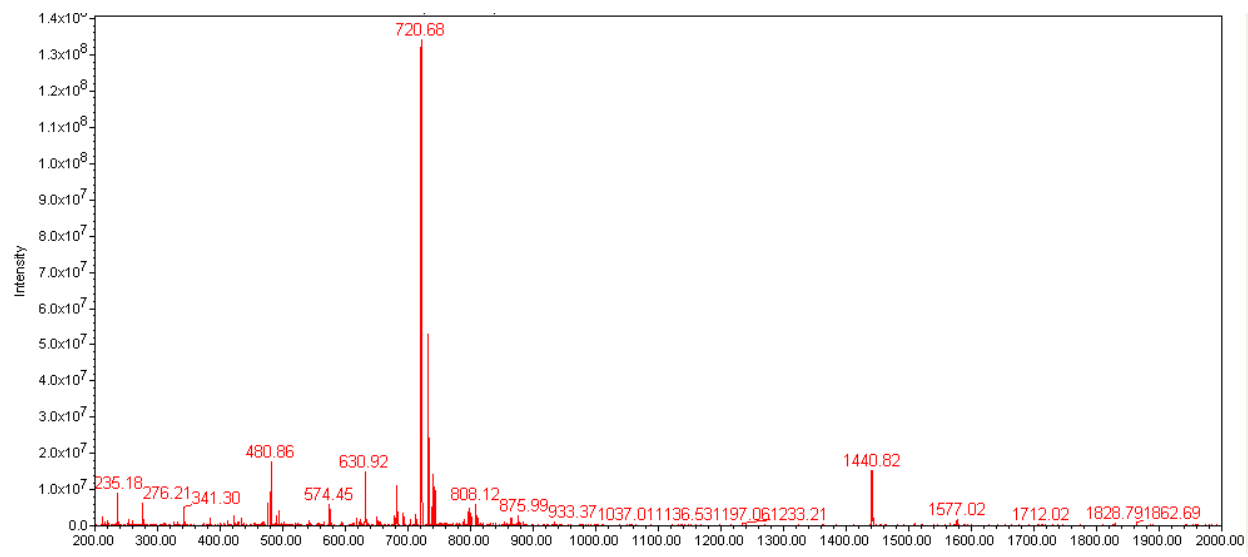

Figure S24. ESI-MS calcd for $\mathrm{C}_{64} \mathrm{H}_{102} \mathrm{~N}_{20} \mathrm{O}_{18}[\mathrm{M}+\mathrm{H}]^{+} \mathrm{m} / z=1439.77$, found 1440.82; $[\mathrm{M}+2 \mathrm{H}]^{2+} \mathrm{m} / z=720.39$, found 720.68

\section{Peptide 2f: $\mathrm{H}_{2} \mathrm{~N}$-SARKLLQDIM-CO-SAL ${ }^{\text {off }}$ ester}

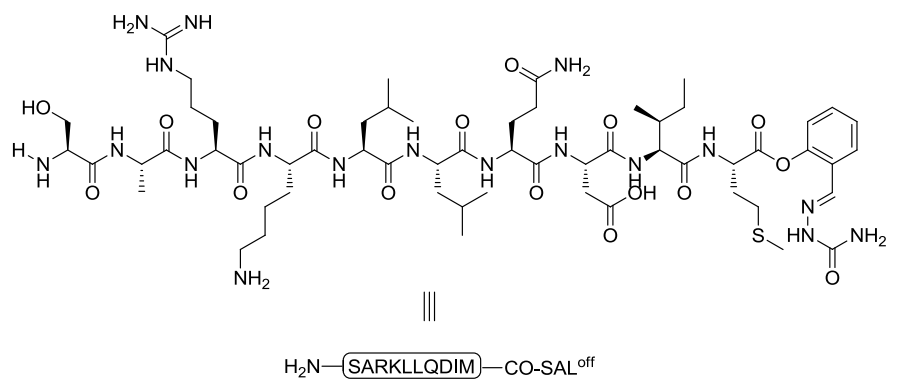

Crude

protected peptide

$B o c H N-S e r\left({ }^{t} \mathrm{Bu}\right)-\mathrm{Ala}-\mathrm{Arg}(\mathrm{Pbf})-\mathrm{Lys}(\mathrm{Boc})-\mathrm{Leu}-\mathrm{Leu}-\mathrm{Gln}(\mathrm{Trt})-\mathrm{Asp}\left(\mathrm{O}^{t} \mathrm{Bu}\right)-\mathrm{Ile}-\mathrm{COOH}$ (prepared according to general experimental procedure A and B) $(50.3 \mathrm{mg}, 27.2 \mu \mathrm{mol})$ was dissolved in $\mathrm{CHCl}_{3}$ /trifluoroethanol $(1.8 \mathrm{~mL})$ and reacted with $\mathbf{H C l} \cdot \boldsymbol{H}_{2} \boldsymbol{N}$-Met-CO-SAL ${ }^{\text {off }}(\mathbf{M})(28.3 \mathrm{mg}, 81.6 \mu \mathrm{mol})$ in the presence of EDC (12.6 $\mathrm{mg}, 81.6 \mu \mathrm{mol})$ and HOOBt $(13.3 \mathrm{mg}, 81.6 \mu \mathrm{mol})$, as described in general procedure D. After stirring for $3 \mathrm{~h}$, the reaction mixture was concentrated and subjected to 5.0 $\mathrm{mL}$ of $\mathrm{TFA} / \mathrm{H}_{2} \mathrm{O}(95 / 5, v / v)$ for $2 \mathrm{~h}$. Preparative HPLC purification (15-35\% $\mathrm{CH}_{3} \mathrm{CN} / \mathrm{H}_{2} \mathrm{O}$ over $30 \mathrm{~min}$ ) followed by concentration at reduced pressure and lyophilization afforded peptide $\mathbf{2 f}(17.1 \mathrm{mg}, 47 \%$ yield) as a white powder. 


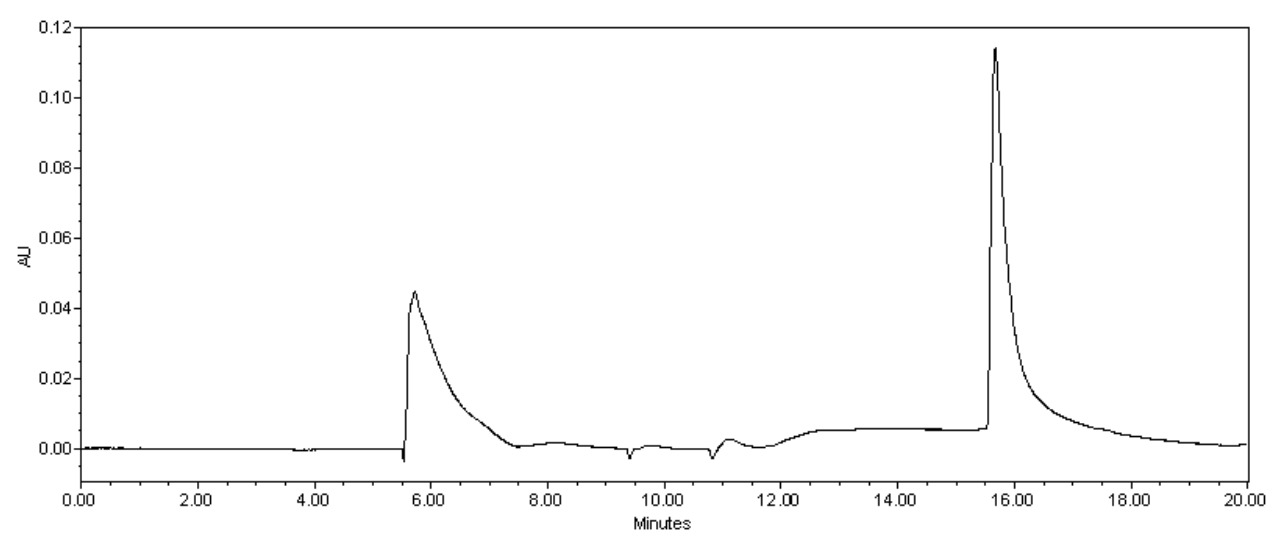

Figure S25. UV trace from analytical LC-MS analysis of purified peptide 2f: gradient $5-95 \% \mathrm{CH}_{3} \mathrm{CN} / \mathrm{H}_{2} \mathrm{O}$ containing $0.1 \%$ TFA over $15 \mathrm{~min}$ at a flow rate of $0.6 \mathrm{~mL} / \mathrm{min}$.

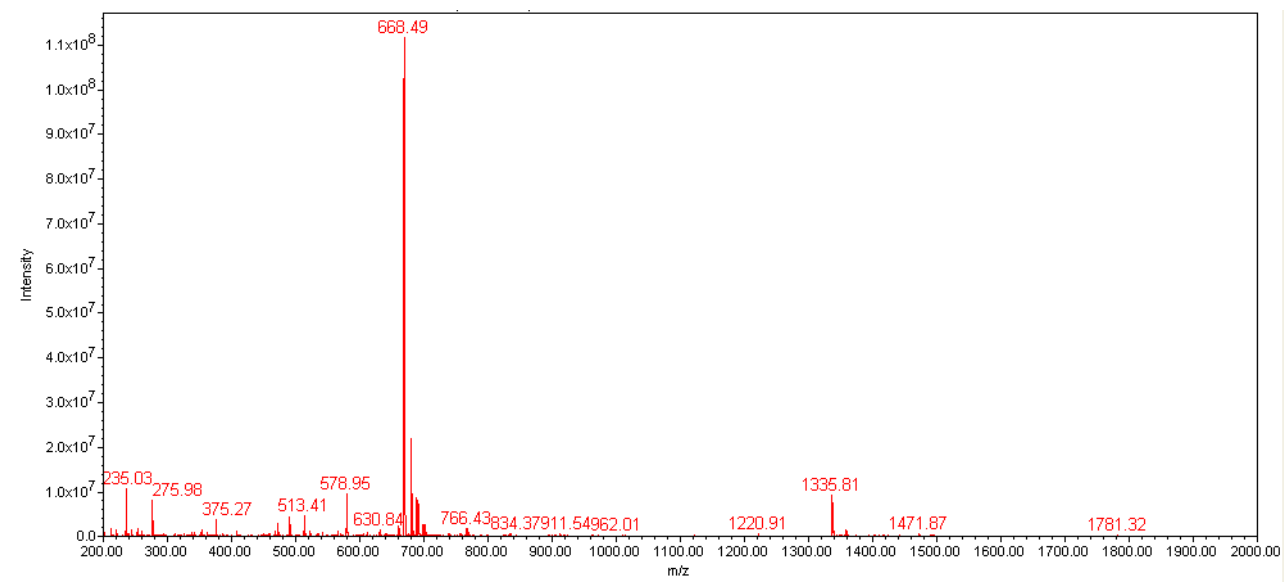

Figure S26. ESI-MS calcd. for $\mathrm{C}_{58} \mathrm{H}_{99} \mathrm{~N}_{18} \mathrm{O}_{16} \mathrm{~S}[\mathrm{M}+\mathrm{H}]^{+} \mathrm{m} / z=1335.72$, found 1335.81; $[\mathrm{M}+2 \mathrm{H}]^{2+} m / z=668.36$, found 668.49 .

\section{$\underline{V}$. Epimerization study of peptide ester generated by $n+1$ strategy}

Both epimeric $\mathrm{H}_{2} \mathrm{~N}$-VIGGVGNA-CO-SAL ${ }^{\text {off }}$ ester (2g) and $\mathrm{H}_{2} \mathrm{~N}$-VIGGVGnA-CO-SAL ${ }^{\text {off }}$ ester (2h) were synthesized by $\mathrm{n}+1$ strategy to demonstrate that no epimerization occurred at the C-terminal Asn residues.

Crude peptide 2g: $\mathrm{H}_{2} \mathrm{~N}$-VIGGVGNA-CO-SAL ${ }^{\text {off }}$ ester 


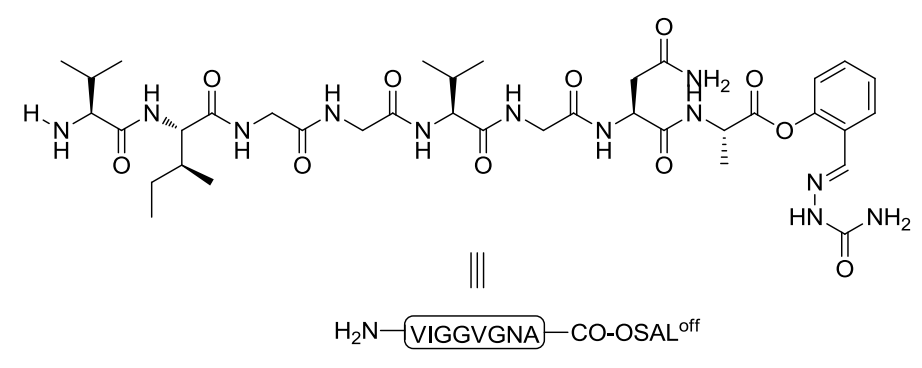

Crude protected peptide BocHN-Val-Ile-Gly-Gly-Val-Gly-Asn(Trt)-COOH (prepared according to general experimental procedure A and B) $(20.2 \mathrm{mg}, 21.1 \mu \mathrm{mol})$ was dissolved in $\mathrm{CHCl}_{3} /$ trifluoroethanol $(1.4 \mathrm{~mL})$ and reacted with $\mathbf{H C l} \cdot \mathrm{H}_{2} \boldsymbol{N}$-Ala-CO-SAL ${ }^{\text {off }}(\mathbf{A})(18.1 \mathrm{mg}, 63.3 \mu \mathrm{mol})$ in the presence of EDC $(9.8 \mathrm{mg}$, $63.3 \mu \mathrm{mol})$ and $\mathrm{HOOBt}(10.3 \mathrm{mg}, 63.3 \mu \mathrm{mol})$, as described in general procedure D. After stirring for $3 \mathrm{~h}$, the reaction mixture was concentrated and subjected to $5.0 \mathrm{~mL}$ of $\mathrm{TFA} / \mathrm{H}_{2} \mathrm{O} \quad(95 / 5, v / v)$ for $1 \mathrm{~h}$ to obtain crude peptide $\mathbf{2 g}$ : $\mathrm{H}_{2} \mathrm{~N}$-VIGGVGNA-CO-SAL ${ }^{\text {off }}$

Crude peptide 2h: $\mathrm{H}_{2} \mathrm{~N}$-VIGGVGnA-CO-SAL ${ }^{\text {off }}$ ester

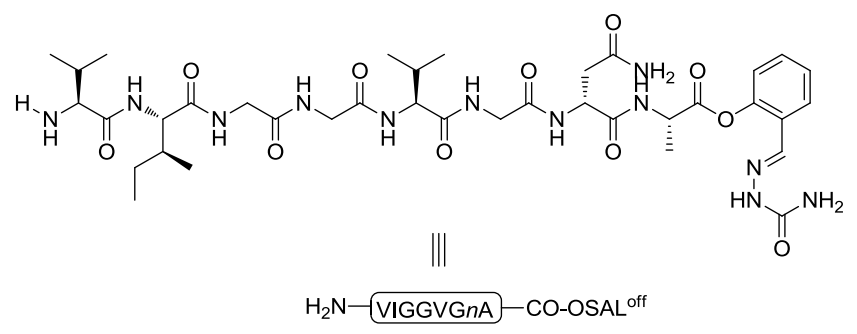

Crude protected peptide BocHN-Val-Ile-Gly-Gly-Val-Gly-asn(Trt)-COOH (prepared according to general experimental procedure A and B) $(20.5 \mathrm{mg}, 21.4 \mu \mathrm{mol})$ was dissolved in $\mathrm{CHCl}_{3}$ /trifluoroethanol $(1.4 \mathrm{~mL})$ and reacted with $\mathbf{H C l} \cdot \mathbf{H}_{2} \boldsymbol{N}$-Ala-CO-SAL ${ }^{\text {off }}(\mathbf{A})(18.4 \mathrm{mg}, 64.3 \mu \mathrm{mol})$ in the presence of EDC (10.0 $\mathrm{mg}$, $64.3 \mu \mathrm{mol})$ and HOOBt $(10.5 \mathrm{mg}, 64.3 \mu \mathrm{mol})$, as described in general procedure D. After stirring for $3 \mathrm{~h}$, the reaction mixture was concentrated and subjected to $5.0 \mathrm{~mL}$ of $\mathrm{TFA} / \mathrm{H}_{2} \mathrm{O} \quad(95 / 5, \quad v / v)$ for $1 \mathrm{~h}$ to obtain crude peptide $\mathbf{2 h}$ : $\mathrm{H}_{2} \mathrm{~N}$-VIGGVGnA-CO-SAL ${ }^{\text {off }}$ 
Crude peptide $\mathbf{2 g}$ and $\mathbf{2 h}$ and a co-injection of a mixture of crude peptide $\mathbf{2 g}$ and $\mathbf{2 h}$ were analyzed separately by LC-MS. No epimerization was observed for $n+1$ strategy.
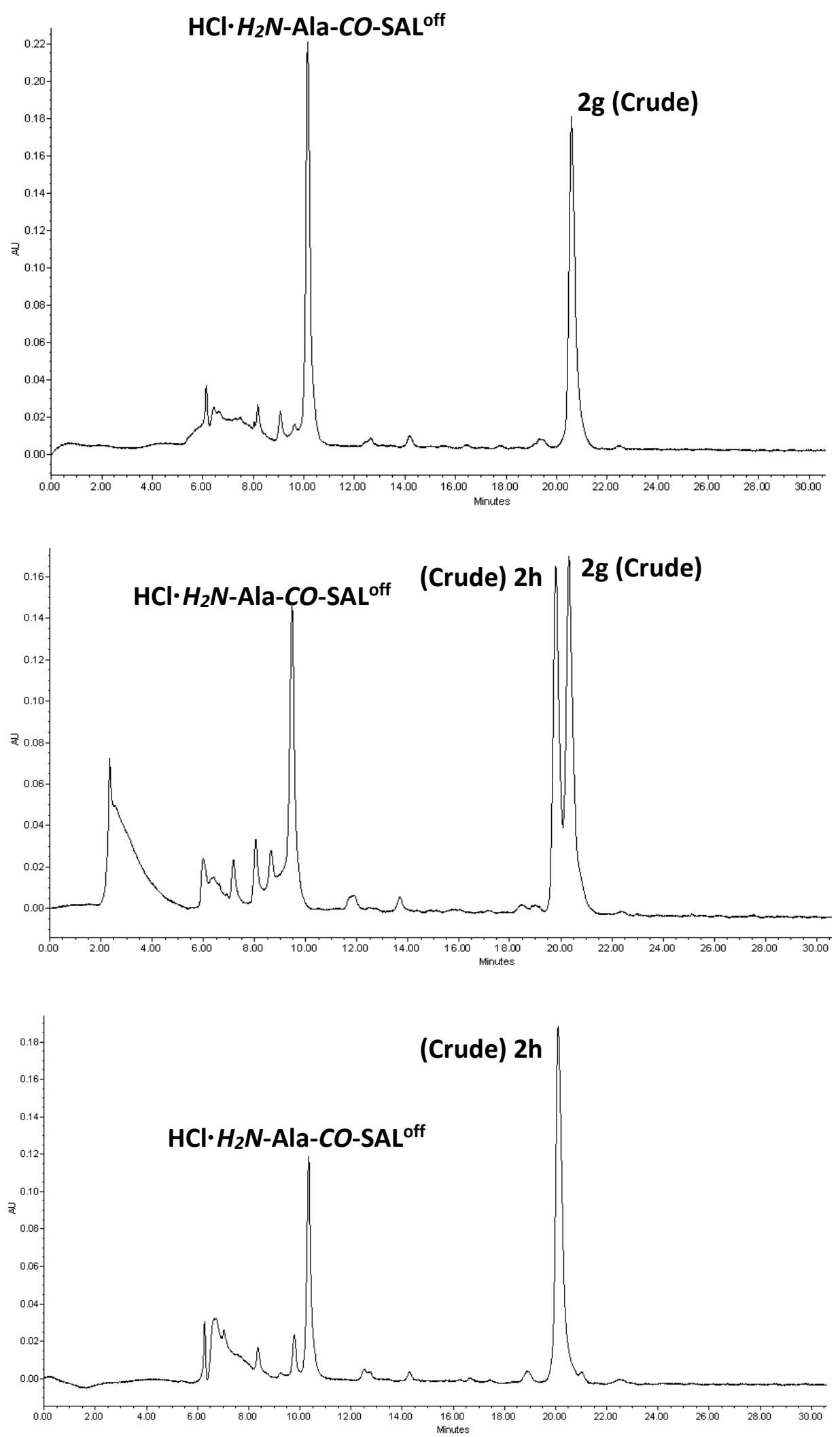
Figure S27. UV trace $(190-400 \mathrm{~nm})$ from LC-MS analysis of crude $\mathbf{2 g}$, a mixture of crude $\mathbf{2 g}$ and $\mathbf{2 h}$ and crude $\mathbf{2 h}$ for epimerization study: gradient $20-30 \% \mathrm{CH}_{3} \mathrm{CN} / \mathrm{H}_{2} \mathrm{O}$ containing $0.1 \%$ TFA over $30 \mathrm{~min}$ at a flow rate of $0.6 \mathrm{~mL} / \mathrm{min}$.

VI. Model $N$-to- $C$ STL study between peptide SAL $^{\text {on }}$ ester and peptide SAL ${ }^{\text {off }}$ ester to generate ligated peptide SAL ${ }^{\text {on }}$ ester through the use of pyruvic acid

Peptide 3a: $\mathrm{H}_{2} \mathrm{~N}$-VIGGVGNATLHAPTDY-CO-SAL ${ }^{\text {on }}$ ester

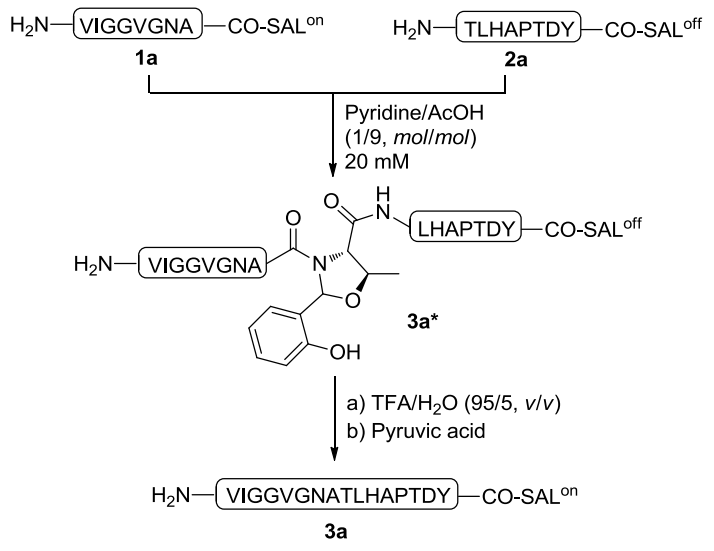

Scheme S1. Model $N$-to- $C$ STL study between peptide 1a and $2 \mathbf{a}$.

Peptide 1a $(5.0 \mathrm{mg}, 6.3 \mu \mathrm{mol})$ and peptide $\mathbf{2 a}(10.2 \mathrm{mg}, 9.5 \mu \mathrm{mol})$ were dissolved in pyridine/acetic acid $(1 / 9, \mathrm{~mol} / \mathrm{mol})$ buffer at a concentration of $20 \mathrm{mM}$ at room temperature. The reaction mixture was stirred at room temperature for $9 \mathrm{~h}$. After completion of the reaction, the solvent was blown off under a stream of condensed air. The residue was then treated with $1.0 \mathrm{~mL}$ of $\mathrm{TFA} / \mathrm{H}_{2} \mathrm{O}(95 / 5, v / v)$ and pyruvic acid (4.5 $\mu \mathrm{L}, 63.4 \mu \mathrm{mol})$. Preparative HPLC purification $\left(10-50 \% \mathrm{CH}_{3} \mathrm{CN} / \mathrm{H}_{2} \mathrm{O}\right.$ over 30 min) followed by concentration at reduced pressure and lyophilization afforded 4.2 mg (39\% yield) of peptide $\mathbf{3 a}$ as a white powder. 

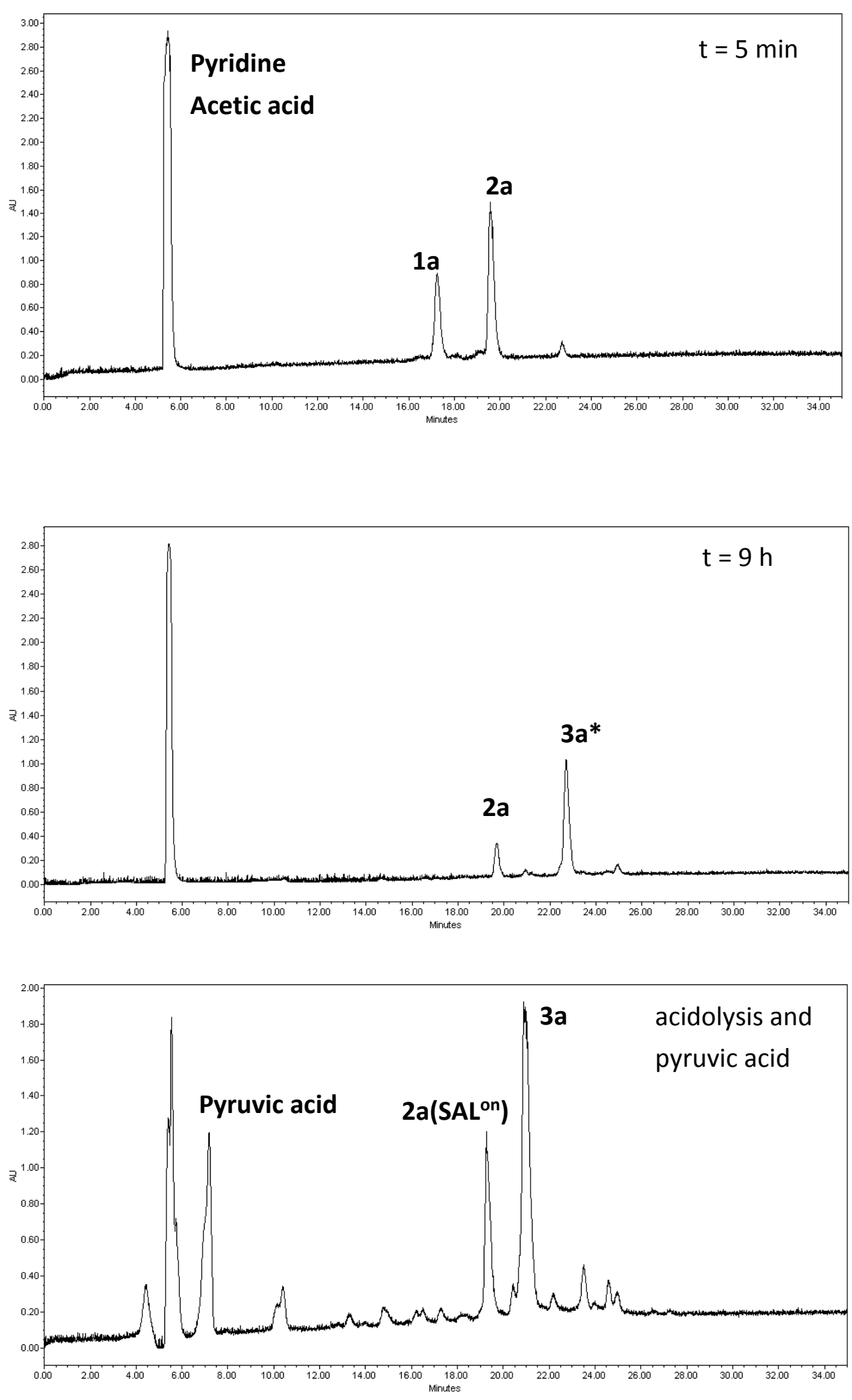

Figure S28. UV trace (190-400 nm) from LC-MS analysis of STL between 1a and 2a at $5 \mathrm{~min}, 9 \mathrm{~h}$ to generate $\mathbf{3} \mathbf{a}^{*}$ and after in situ acidolysis and pyruvic acid to give $\mathbf{3 a}$ : gradient $10-50 \% \mathrm{CH}_{3} \mathrm{CN} / \mathrm{H}_{2} \mathrm{O}$ containing $0.1 \%$ TFA over $30 \mathrm{~min}$ at a flow rate of 0.6 $\mathrm{mL} / \mathrm{min}$. 


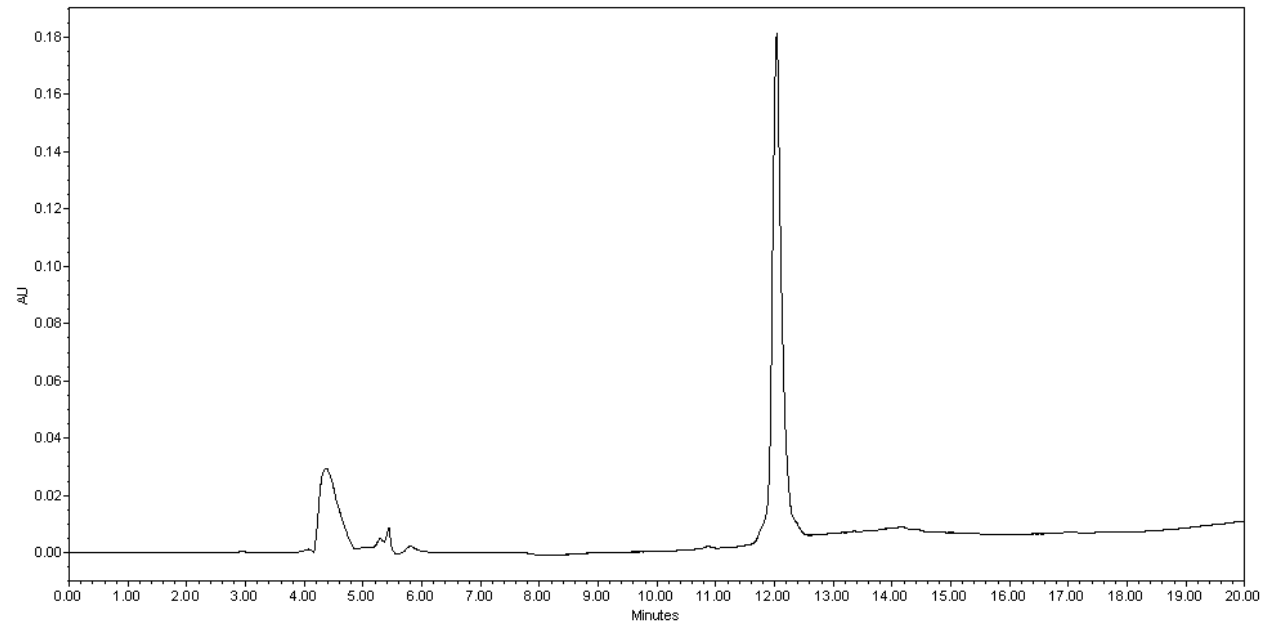

Figure S29. UV trace from analytical LC-MS analysis of purified peptide 3a: gradient $5-95 \% \mathrm{CH}_{3} \mathrm{CN} / \mathrm{H}_{2} \mathrm{O}$ containing $0.1 \%$ TFA over $15 \mathrm{~min}$ at a flow rate of 0.6 $\mathrm{mL} / \mathrm{min}$.

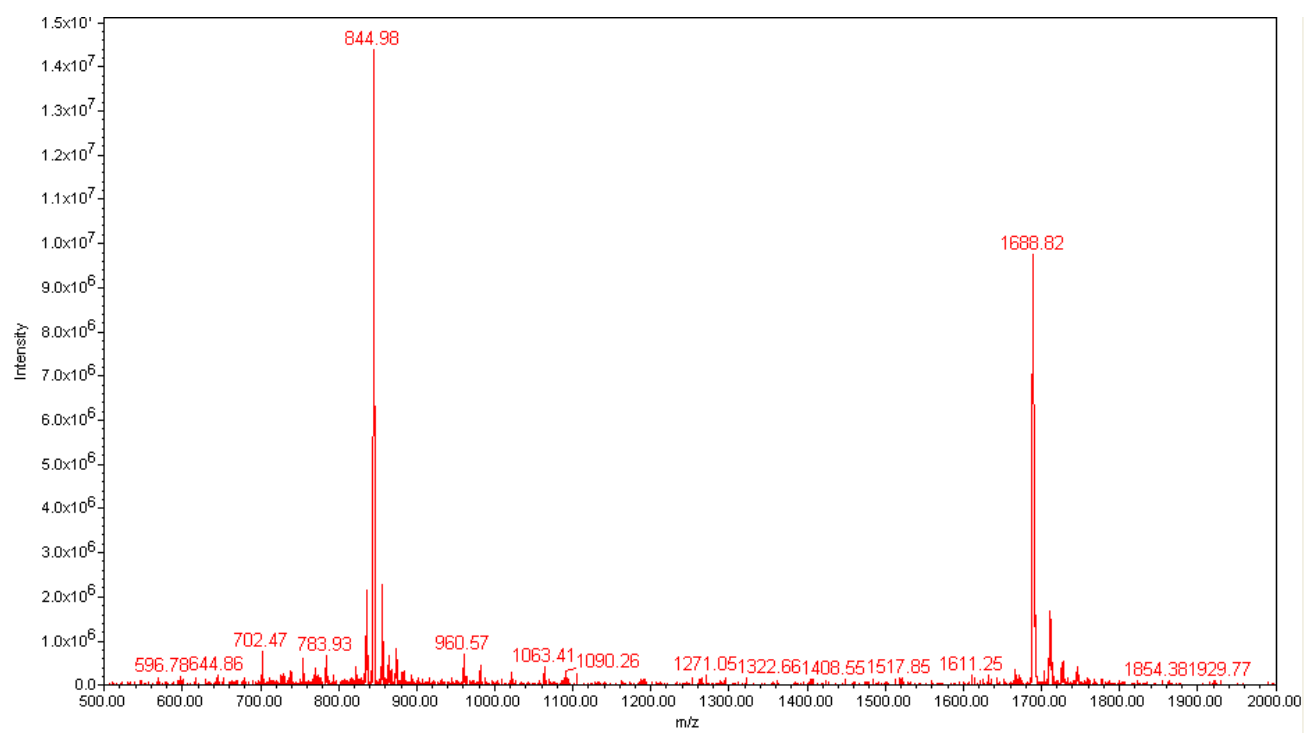

Figure S30. ESI-MS calcd. for $\mathrm{C}_{77} \mathrm{H}_{114} \mathrm{~N}_{19} \mathrm{O}_{24}[\mathrm{M}+\mathrm{H}]^{+} \mathrm{m} / z=1688.83$, found 1688.82; $[\mathrm{M}+2 \mathrm{H}]^{2+} m / z=844.92$, found 844.98 


\section{Peptide 3b: $\mathrm{H}_{2} \mathrm{~N}$-VIGGVGNYTLHAPTDS-CO-SAL ${ }^{\text {on }}$ ester}

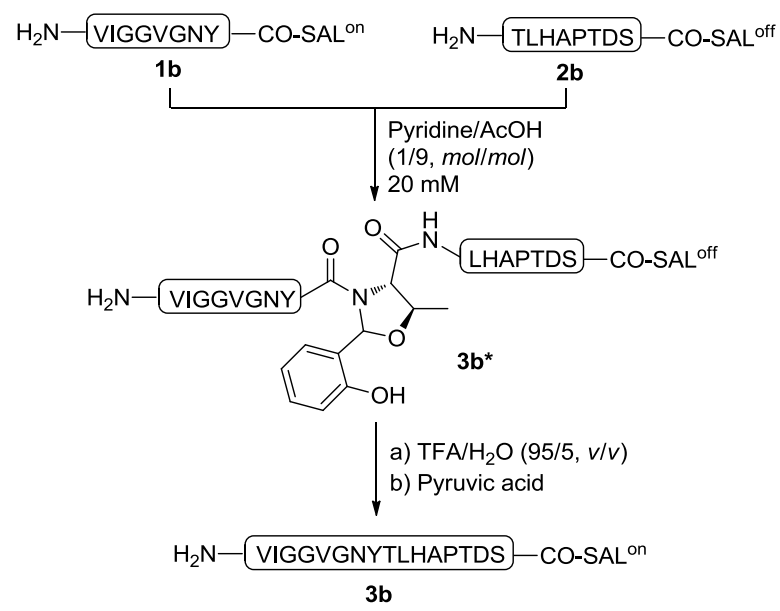

Scheme S2. Model $N$-to- $C$ STL study between peptide $\mathbf{1 b}$ and $\mathbf{2 b}$.

Peptide $\mathbf{1 b}(5.0 \mathrm{mg}, 5.7 \mu \mathrm{mol})$ and peptide $\mathbf{2 b}(8.5 \mathrm{mg}, 8.5 \mu \mathrm{mol})$ were dissolved in pyridine/acetic acid $(1 / 9, \mathrm{~mol} / \mathrm{mol})$ buffer at a concentration of $20 \mathrm{mM}$ at room temperature. The reaction mixture was stirred at room temperature for $3 \mathrm{~h}$. After completion of the reaction, the solvent was blown off under a stream of condensed air. The residue was then treated with $1.0 \mathrm{~mL}$ of $\mathrm{TFA} / \mathrm{H}_{2} \mathrm{O}(95 / 5, v / v)$ and pyruvic acid $(4.0 \mu \mathrm{L}, 56.8 \mu \mathrm{mol})$. Preparative HPLC purification $\left(10-50 \% \mathrm{CH}_{3} \mathrm{CN} / \mathrm{H}_{2} \mathrm{O}\right.$ over 30 min) followed by concentration at reduced pressure and lyophilization afforded 2.9 mg (30\% yield) of peptide $\mathbf{3 b}$ as a white powder. 

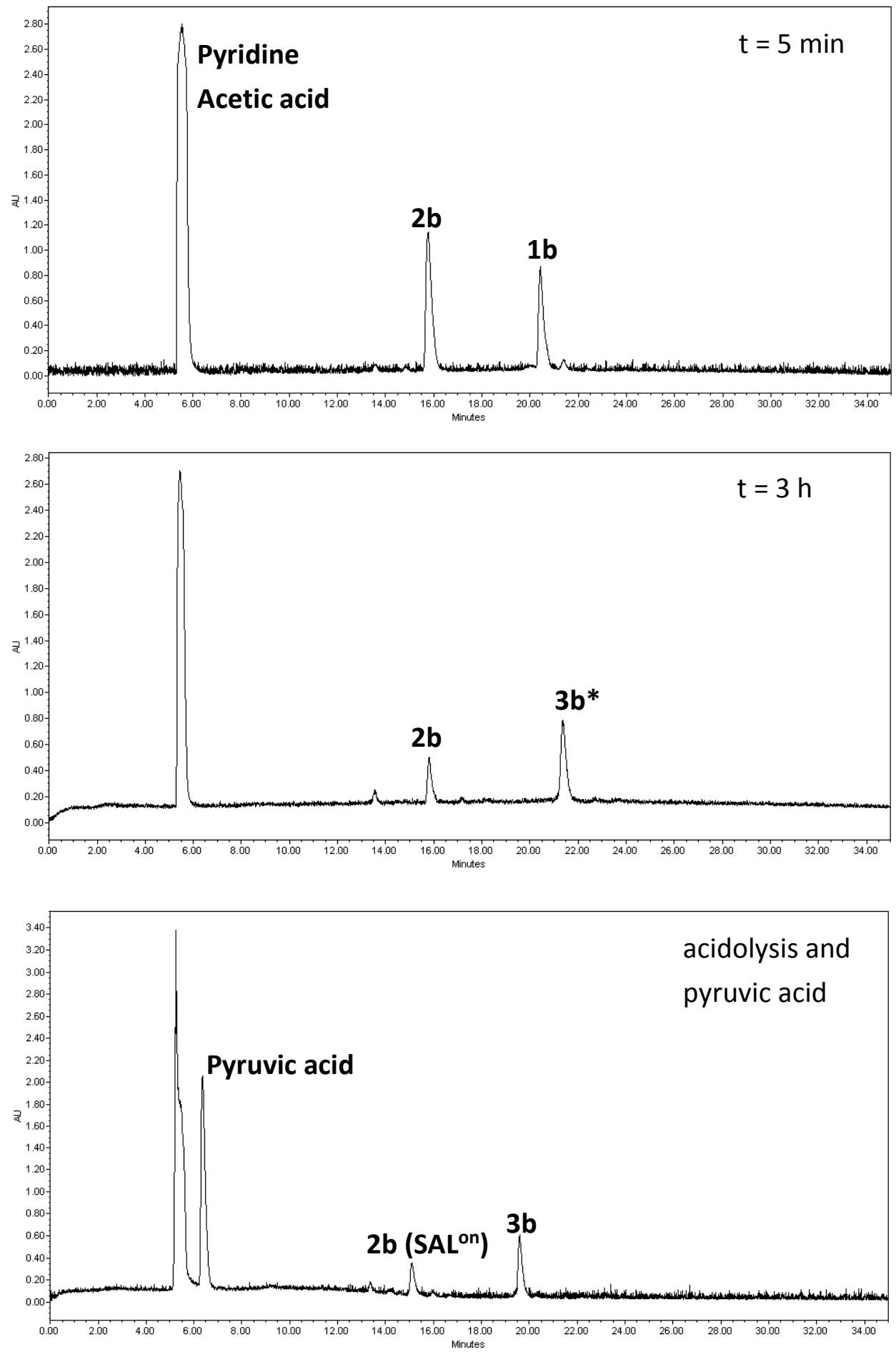

Figure S31. UV trace (190-400 nm) from LC-MS analysis of STL between $\mathbf{1 b}$ and $\mathbf{2 b}$ at $5 \mathrm{~min}, 3 \mathrm{~h}$ to generate $\mathbf{3} \mathbf{b}^{*}$ and after in situ acidolysis and pyruvic acid to give $\mathbf{3 b}$ : gradient $10-50 \% \mathrm{CH}_{3} \mathrm{CN} / \mathrm{H}_{2} \mathrm{O}$ containing $0.1 \%$ TFA over $30 \mathrm{~min}$ at a flow rate of 0.6 $\mathrm{mL} / \mathrm{min}$. 


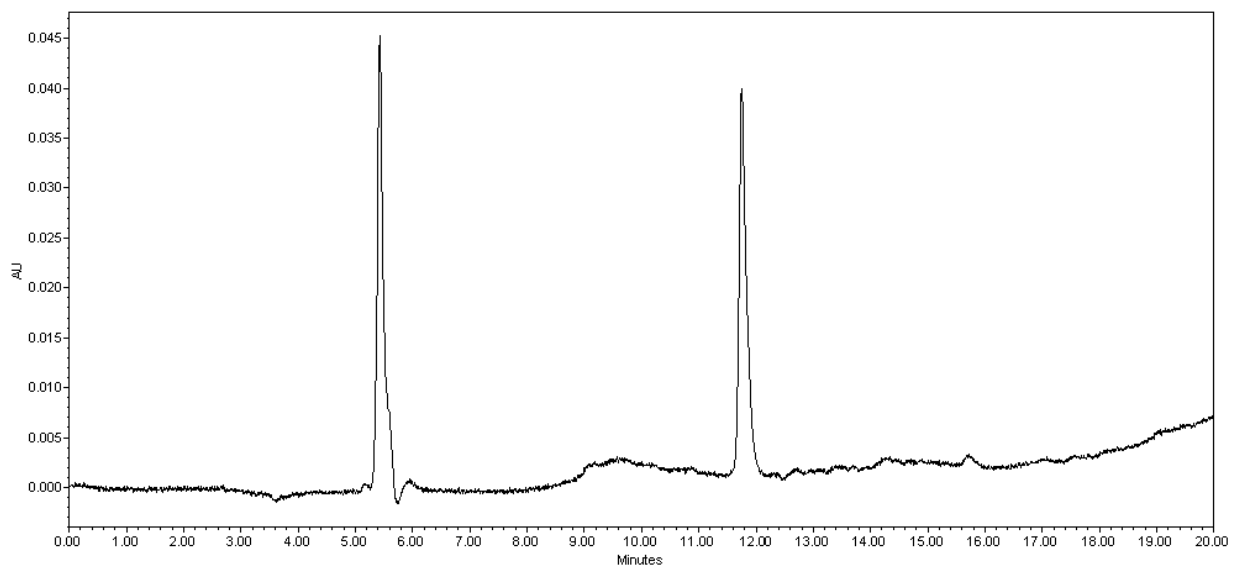

Figure S32. UV trace from analytical LC-MS analysis of purified peptide 3b: gradient $5-95 \% \mathrm{CH}_{3} \mathrm{CN} / \mathrm{H}_{2} \mathrm{O}$ containing $0.1 \%$ TFA over $15 \mathrm{~min}$ at a flow rate of 0.6 $\mathrm{mL} / \mathrm{min}$.

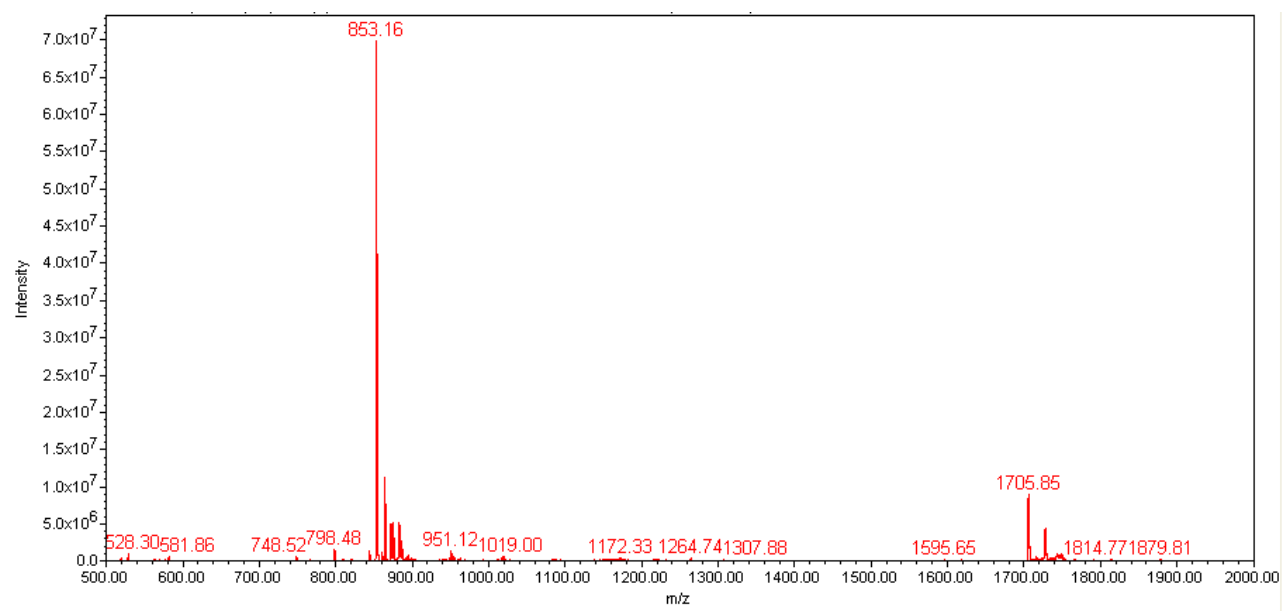

Figure S33. ESI-MS calcd. for $\mathrm{C}_{77} \mathrm{H}_{114} \mathrm{~N}_{19} \mathrm{O}_{25}[\mathrm{M}+\mathrm{H}]^{+} m / z=1705.84$, found 1705.85; $[\mathrm{M}+2 \mathrm{H}]^{2+} \mathrm{m} / \mathrm{z}=853.42$, found 853.16. 
Peptide 3c: $\mathrm{H}_{2} \mathrm{~N}$-VIGGVGNVTLHAPTDA-CO-SAL ${ }^{\text {on }}$ ester

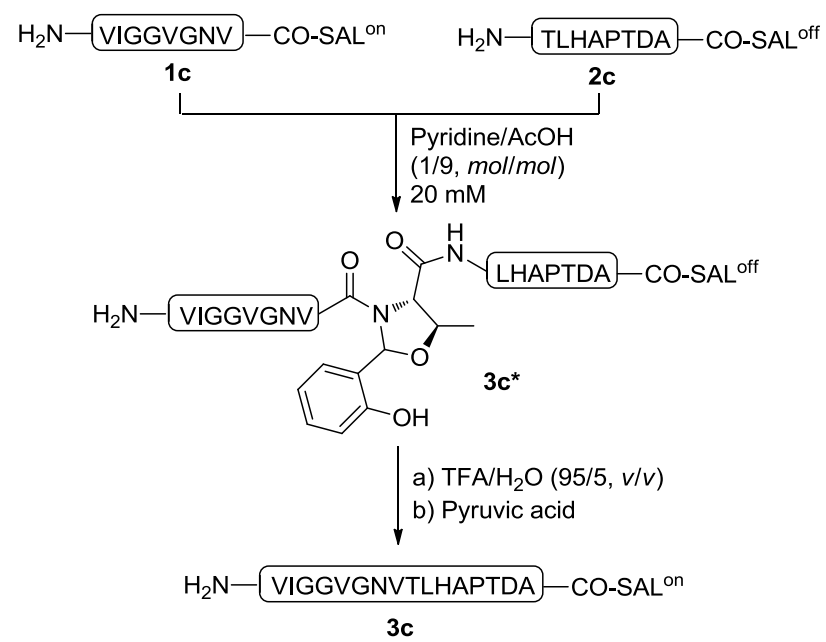

Scheme S3. Model N-to-C STL study between peptide 1c and 2c.

Peptide 1c $(5.0 \mathrm{mg}, 6.1 \mu \mathrm{mol})$ and peptide $2 \mathrm{c}(9.0 \mathrm{mg}, 9.2 \mu \mathrm{mol})$ were dissolved in pyridine/acetic acid $(1 / 9, \mathrm{~mol} / \mathrm{mol})$ buffer at a concentration of $20 \mathrm{mM}$ at room temperature. The reaction mixture was stirred at room temperature for $9 \mathrm{~h}$. After completion of the reaction, the solvent was blown off under a stream of condensed air. The residue was then treated with $1.0 \mathrm{~mL}$ of $\mathrm{TFA} / \mathrm{H}_{2} \mathrm{O}(95 / 5, v / v)$ and pyruvic acid $(4.2 \mu \mathrm{L}, 60.2 \mu \mathrm{mol})$. Preparative HPLC purification $\left(10-50 \% \mathrm{CH}_{3} \mathrm{CN} / \mathrm{H}_{2} \mathrm{O}\right.$ over 30 min) followed by concentration at reduced pressure and lyophilization afforded 3.4 mg (34\% yield) of peptide 3a as a white powder. 

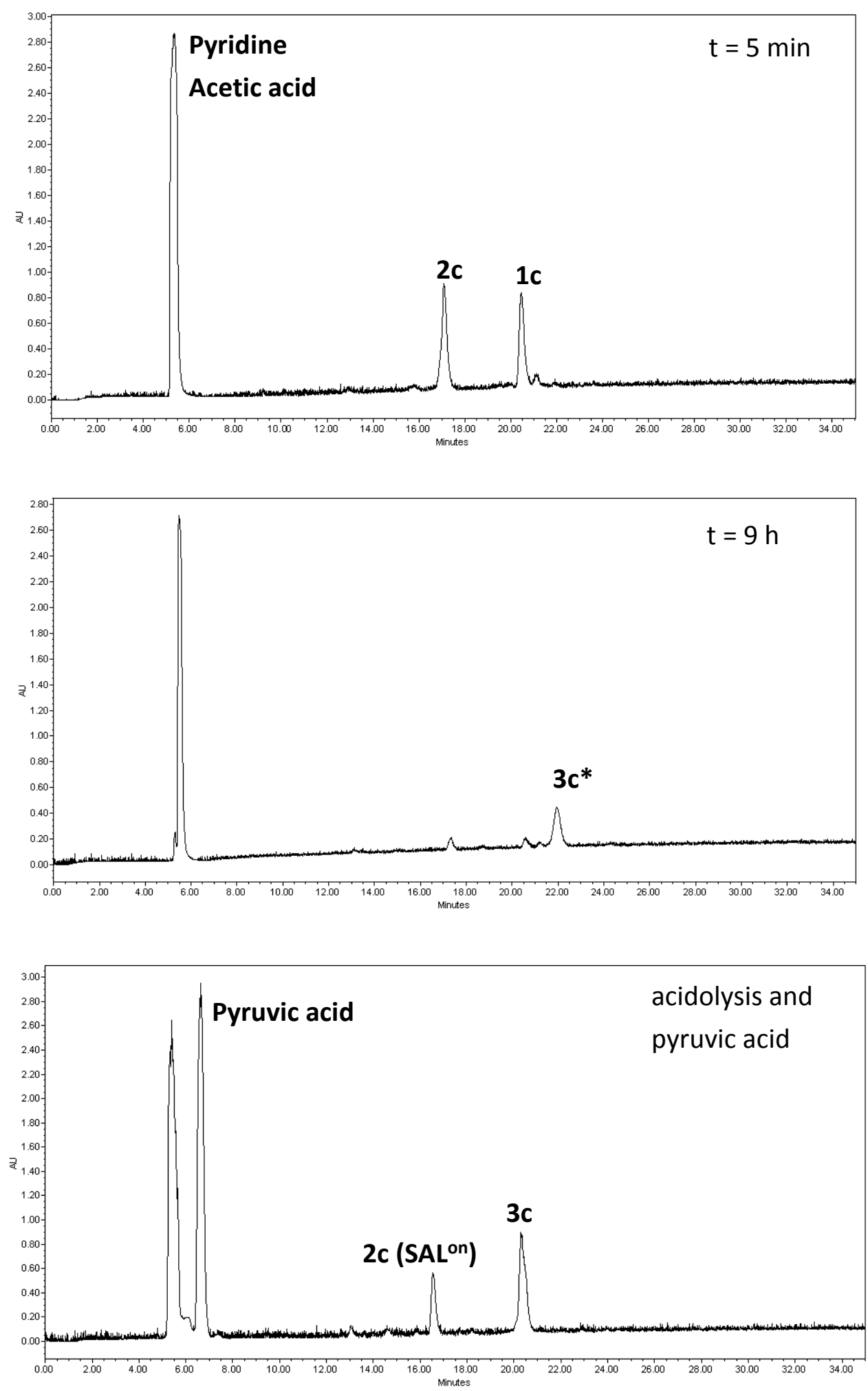

Figure S34. UV trace (190-400 nm) from LC-MS analysis of STL between 1c and 2c at $5 \mathrm{~min}, 9 \mathrm{~h}$ to generate $\mathbf{3} \mathrm{c}^{*}$ and after in situ acidolysis and pyruvic acid to give $\mathbf{3 c}$ : gradient $10-50 \% \mathrm{CH}_{3} \mathrm{CN} / \mathrm{H}_{2} \mathrm{O}$ containing $0.1 \%$ TFA over $30 \mathrm{~min}$ at a flow rate of 0.6 $\mathrm{mL} / \mathrm{min}$. 


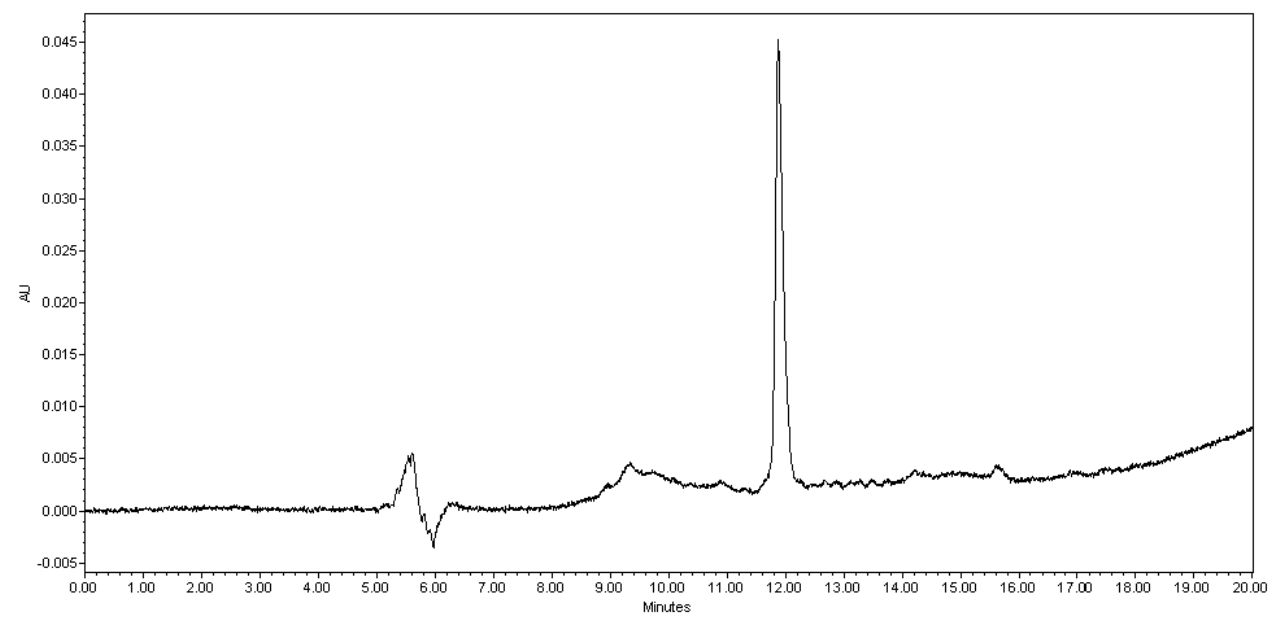

Figure S35. UV trace from analytical LC-MS analysis of purified peptide 3c: gradient $5-95 \% \mathrm{CH}_{3} \mathrm{CN} / \mathrm{H}_{2} \mathrm{O}$ containing $0.1 \%$ TFA over $15 \mathrm{~min}$ at a flow rate of $0.6 \mathrm{~mL} / \mathrm{min}$.

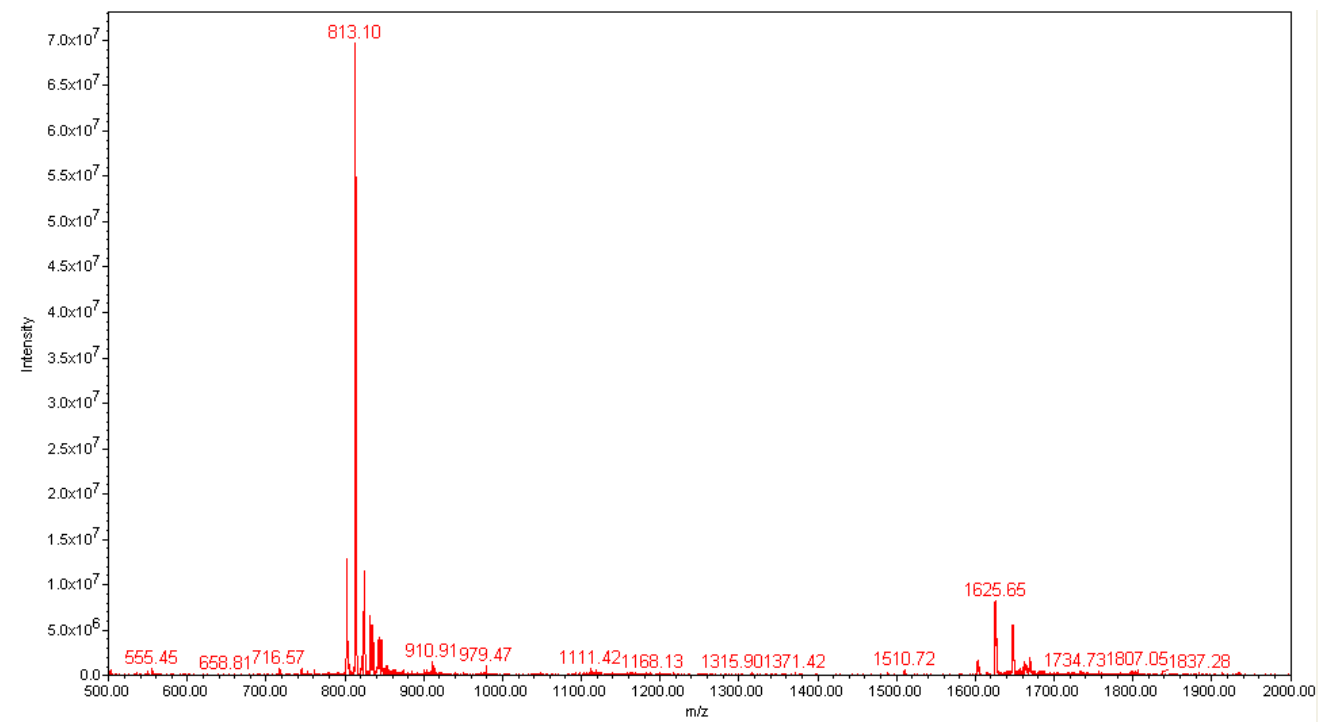

Figure S36. ESI-MS calcd. for $\mathrm{C}_{73} \mathrm{H}_{114} \mathrm{~N}_{19} \mathrm{O}_{23}[\mathrm{M}+\mathrm{H}]^{+} \mathrm{m} / z=1625.80$, found 1625.65; $[\mathrm{M}+2 \mathrm{H}]^{2+} \mathrm{m} / \mathrm{z}=813.40$, found 813.10. 


\section{Peptide 3d: $\mathrm{H}_{2} \mathrm{~N}$-VIGGVGNSTLHAPTDV-CO-SAL ${ }^{\text {on }}$ ester}

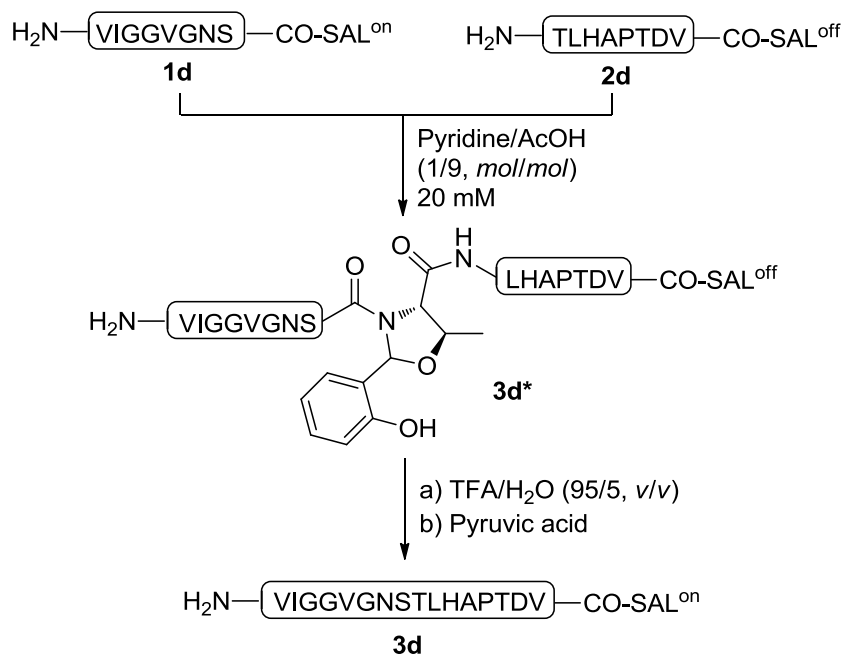

Scheme S4. Model $N$-to- $C$ STL study between peptide $\mathbf{1 d}$ and $\mathbf{2 d}$.

Peptide 1d (5.0 mg, $6.2 \mu \mathrm{mol})$ and peptide 2d (9.4 mg, $9.3 \mu \mathrm{mol})$ were dissolved in pyridine/acetic acid $(1 / 9, \mathrm{~mol} / \mathrm{mol})$ buffer at a concentration of $20 \mathrm{mM}$ at room temperature. The reaction mixture was stirred at room temperature for $2 \mathrm{~h}$. After completion of the reaction, the solvent was blown off under a stream of condensed air. The residue was then treated with $1.0 \mathrm{~mL}$ of $\mathrm{TFA} / \mathrm{H}_{2} \mathrm{O}(95 / 5, v / v)$ and pyruvic acid (4.4 $\mu \mathrm{L}, 60.2 \mu \mathrm{mol})$. Preparative HPLC purification $\left(10-50 \% \mathrm{CH}_{3} \mathrm{CN} / \mathrm{H}_{2} \mathrm{O}\right.$ over 30 min) followed by concentration at reduced pressure and lyophilization afforded 4.3 mg (42\% yield) of peptide $\mathbf{3 d}$ as a white powder. 

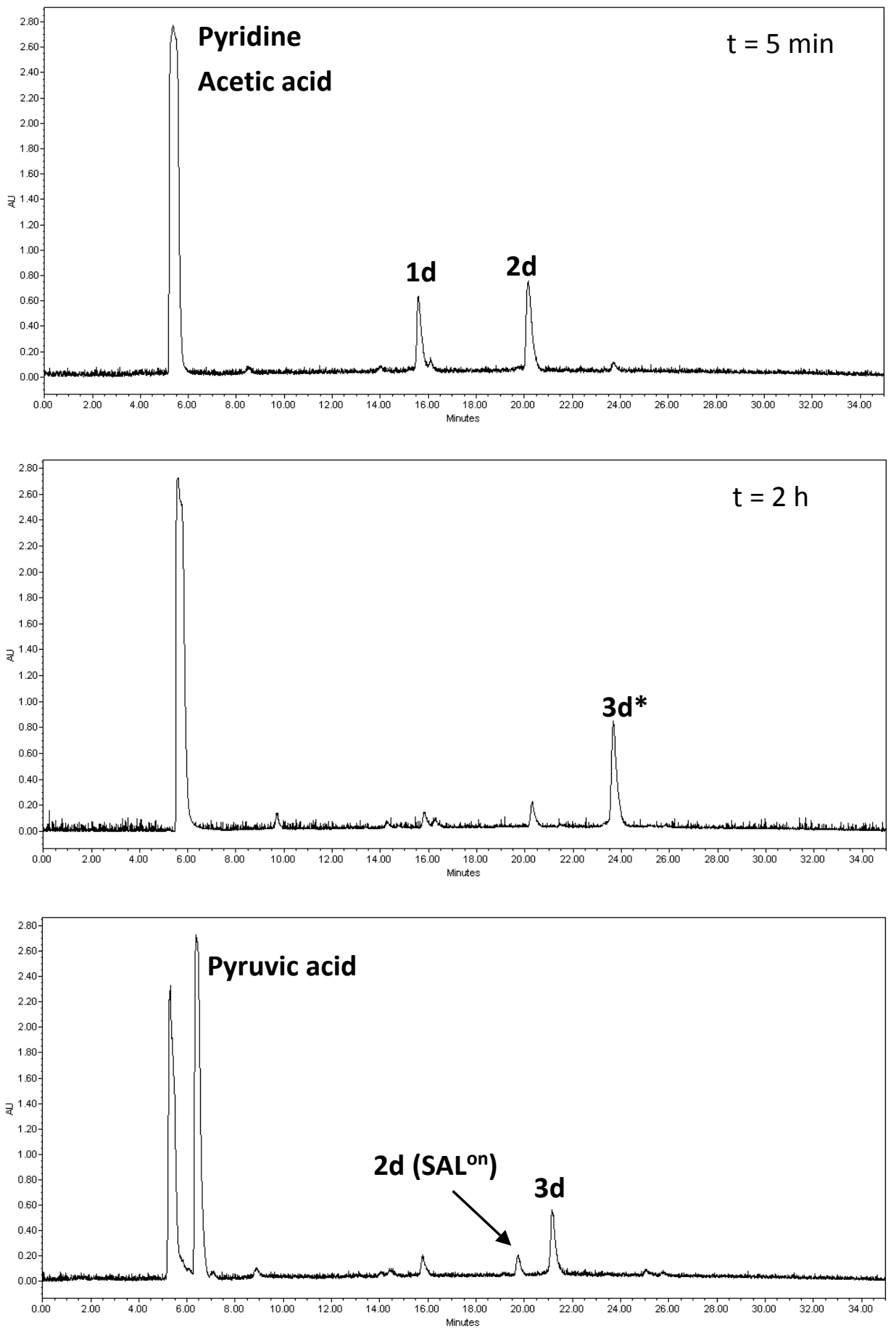

Figure S37. UV trace (190-400 nm) from LC-MS analysis of STL between 1d and 2d at $5 \mathrm{~min}, 2 \mathrm{~h}$ to generate $\mathbf{3 d} *$ and after in situ acidolysis and pyruvic acid to give $\mathbf{3 d}$ : gradient $10-50 \% \mathrm{CH}_{3} \mathrm{CN} / \mathrm{H}_{2} \mathrm{O}$ containing $0.1 \%$ TFA over $30 \mathrm{~min}$ at a flow rate of 0.6 $\mathrm{mL} / \mathrm{min}$. 


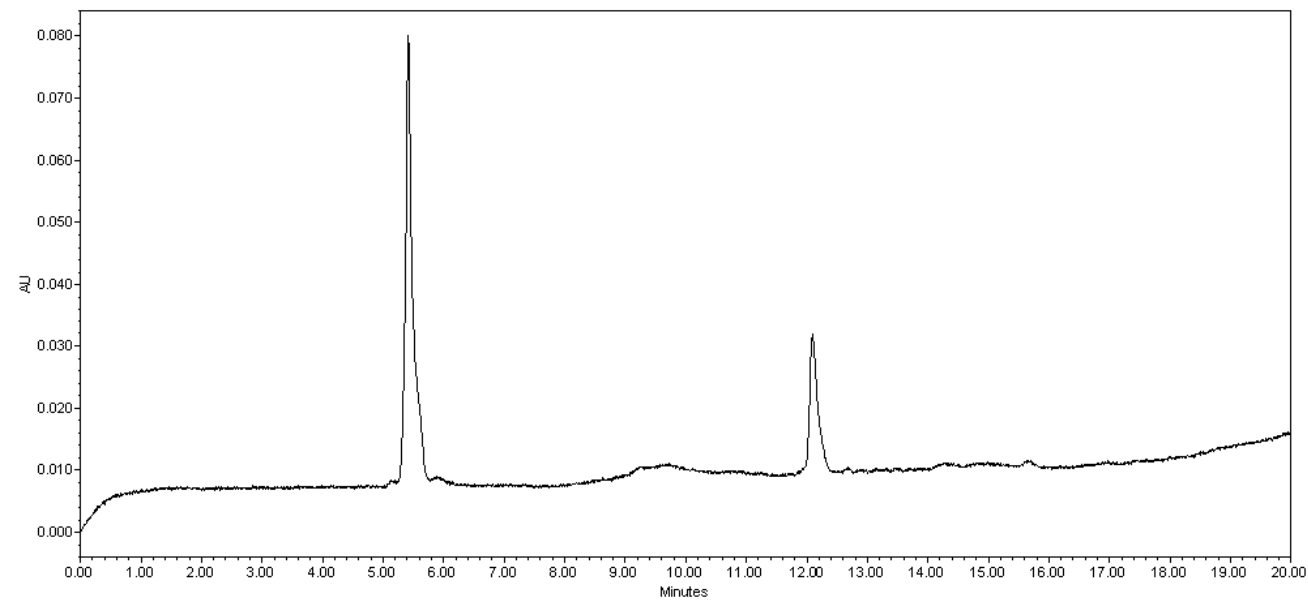

Figure S38. UV trace from analytical LC-MS analysis of purified peptide 3d:

gradient 5-95\% $\mathrm{CH}_{3} \mathrm{CN} / \mathrm{H}_{2} \mathrm{O}$ containing $0.1 \%$ TFA over $15 \mathrm{~min}$ at a flow rate of 0.6 $\mathrm{mL} / \mathrm{min}$.

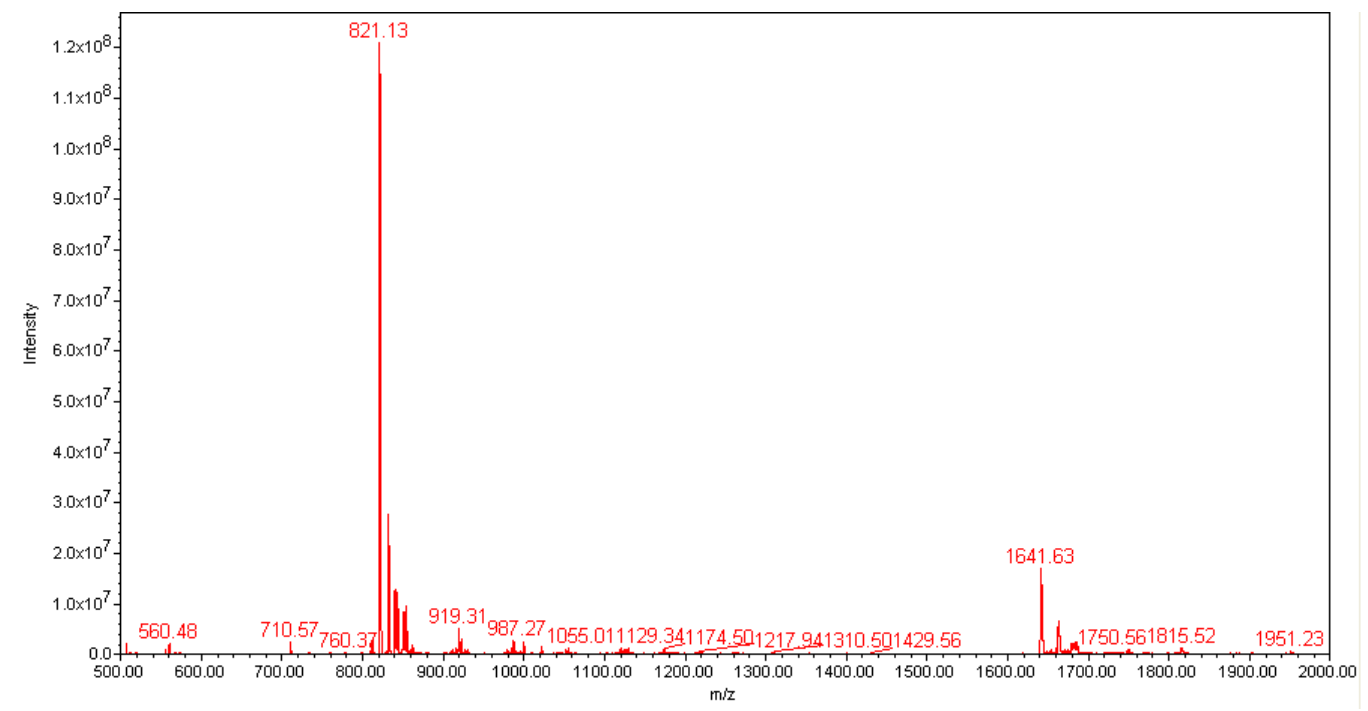

Figure S39. ESI-MS calcd. for $\mathrm{C}_{73} \mathrm{H}_{114} \mathrm{~N}_{19} \mathrm{O}_{24}[\mathrm{M}+\mathrm{H}]^{+} \mathrm{m} / z=1641.80$, found 1641.63; $[\mathrm{M}+2 \mathrm{H}]^{2+} \mathrm{m} / \mathrm{z}=821.40$, found 821.13 . 


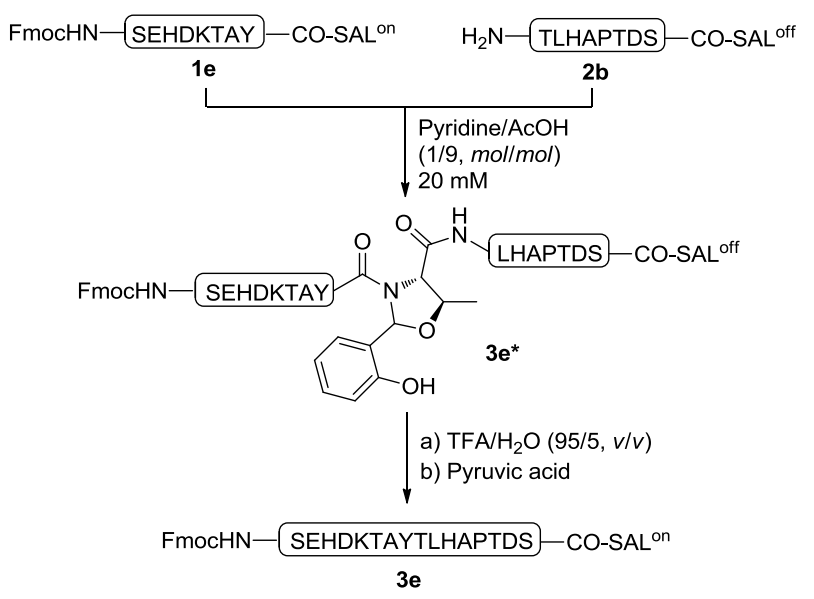

Scheme S5. Model $N$-to- $C$ STL study between peptide $\mathbf{1 e}$ and $\mathbf{2 b}$.

Peptide 1e $(5.0 \mathrm{mg}, 3.9 \mu \mathrm{mol})$ and peptide $\mathbf{2 b}(5.9 \mathrm{mg}, 5.9 \mu \mathrm{mol})$ were dissolved in pyridine/acetic acid $(1 / 9, \mathrm{~mol} / \mathrm{mol})$ buffer at a concentration of $20 \mathrm{mM}$ at room temperature. The reaction mixture was stirred at room temperature for $3 \mathrm{~h}$. After completion of the reaction, the solvent was blown off under a stream of condensed air. The residue was then treated with $1.0 \mathrm{~mL}$ of $\mathrm{TFA} / \mathrm{H}_{2} \mathrm{O}(95 / 5, v / v)$ and pyruvic acid $(2.8 \mu \mathrm{L}, 39.1 \mu \mathrm{mol})$. Preparative HPLC purification $\left(10-50 \% \mathrm{CH}_{3} \mathrm{CN} / \mathrm{H}_{2} \mathrm{O}\right.$ over 30 min) followed by concentration at reduced pressure and lyophilization afforded 5.1 $\mathrm{mg}(62 \%$ yield $)$ of peptide $\mathbf{3 e}$ as a white powder. 

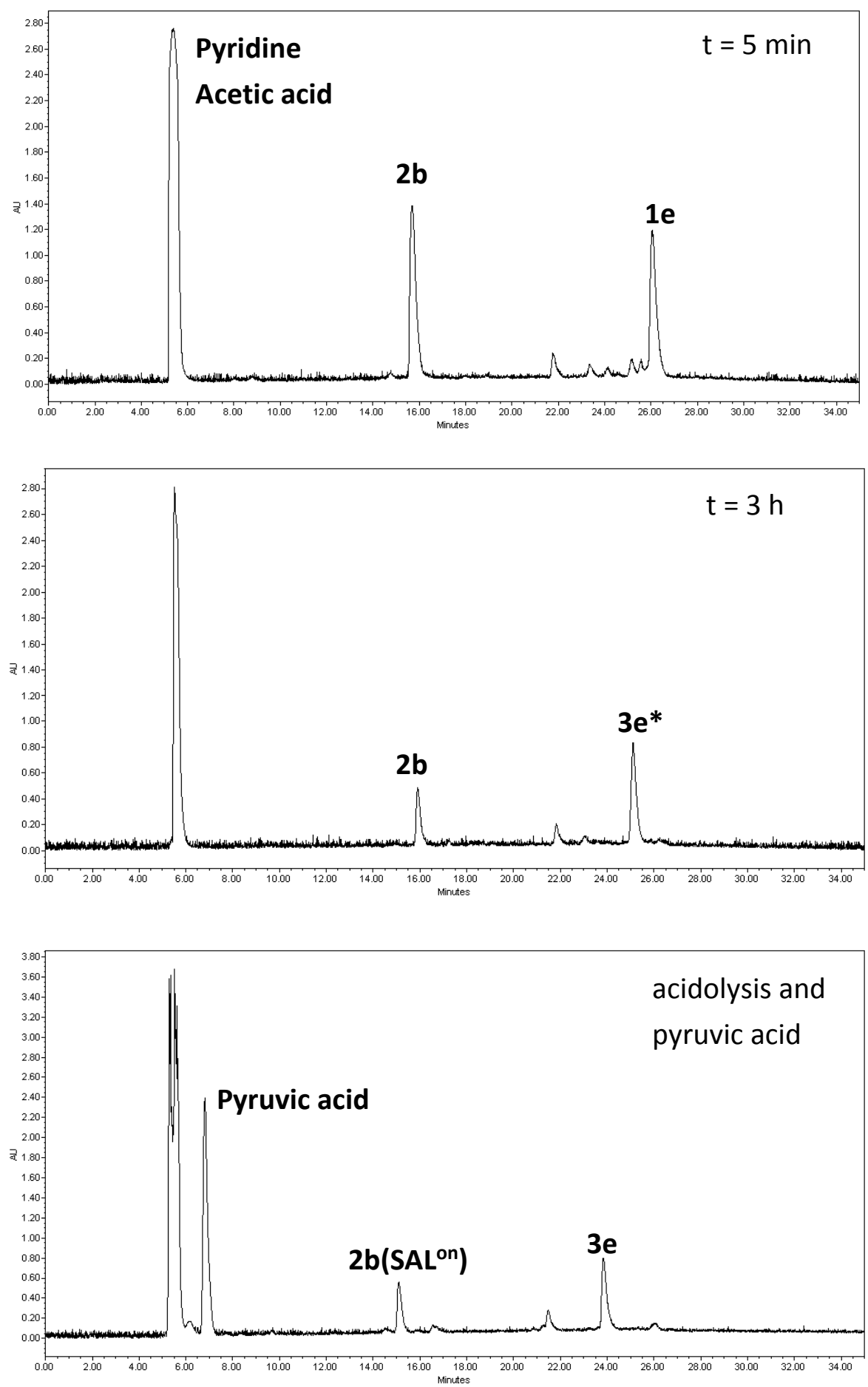

Figure S40. UV trace (190-400 nm) from LC-MS analysis of STL between $\mathbf{1 e}$ and $\mathbf{2 b}$ at $5 \mathrm{~min}, 3 \mathrm{~h}$ to generate $3 \mathrm{e}^{*}$ and after in situ acidolysis and pyruvic acid to give $\mathbf{3 e}$ : gradient $10-50 \% \mathrm{CH}_{3} \mathrm{CN} / \mathrm{H}_{2} \mathrm{O}$ containing $0.1 \%$ TFA over $30 \mathrm{~min}$ at a flow rate of 0.6 $\mathrm{mL} / \mathrm{min}$. 


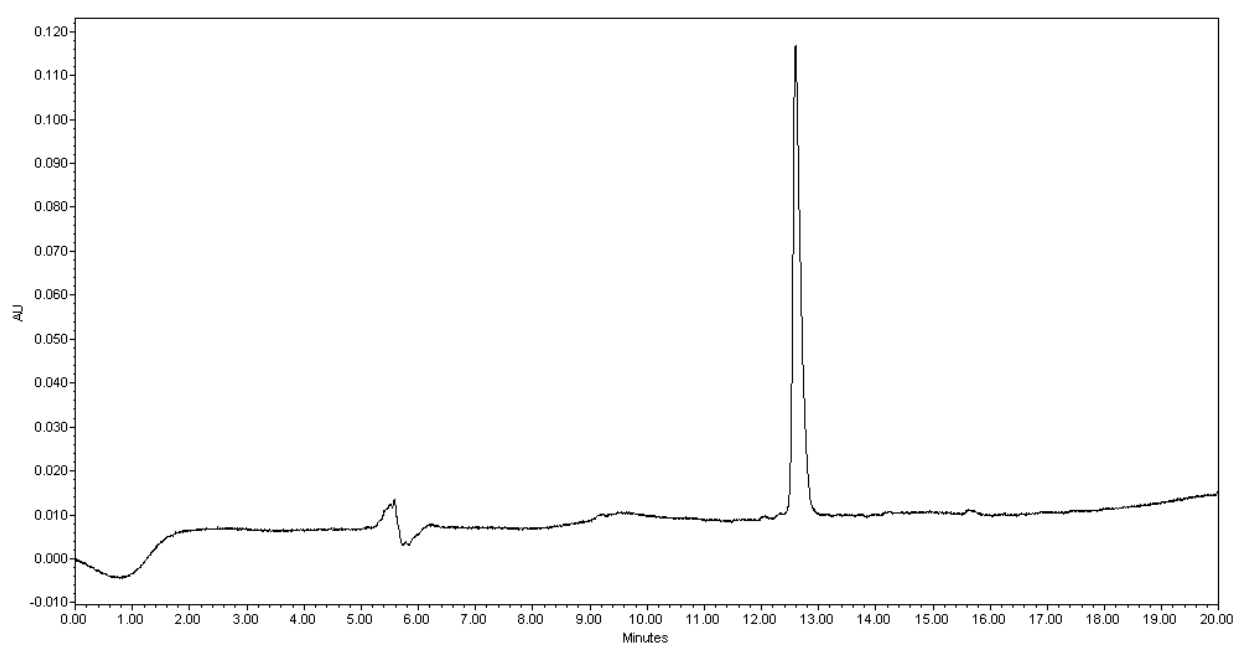

Figure S41. UV trace from analytical LC-MS analysis of purified peptide 3e: gradient $5-95 \% \mathrm{CH}_{3} \mathrm{CN} / \mathrm{H}_{2} \mathrm{O}$ containing $0.1 \%$ TFA over $15 \mathrm{~min}$ at a flow rate of $0.6 \mathrm{~mL} / \mathrm{min}$.

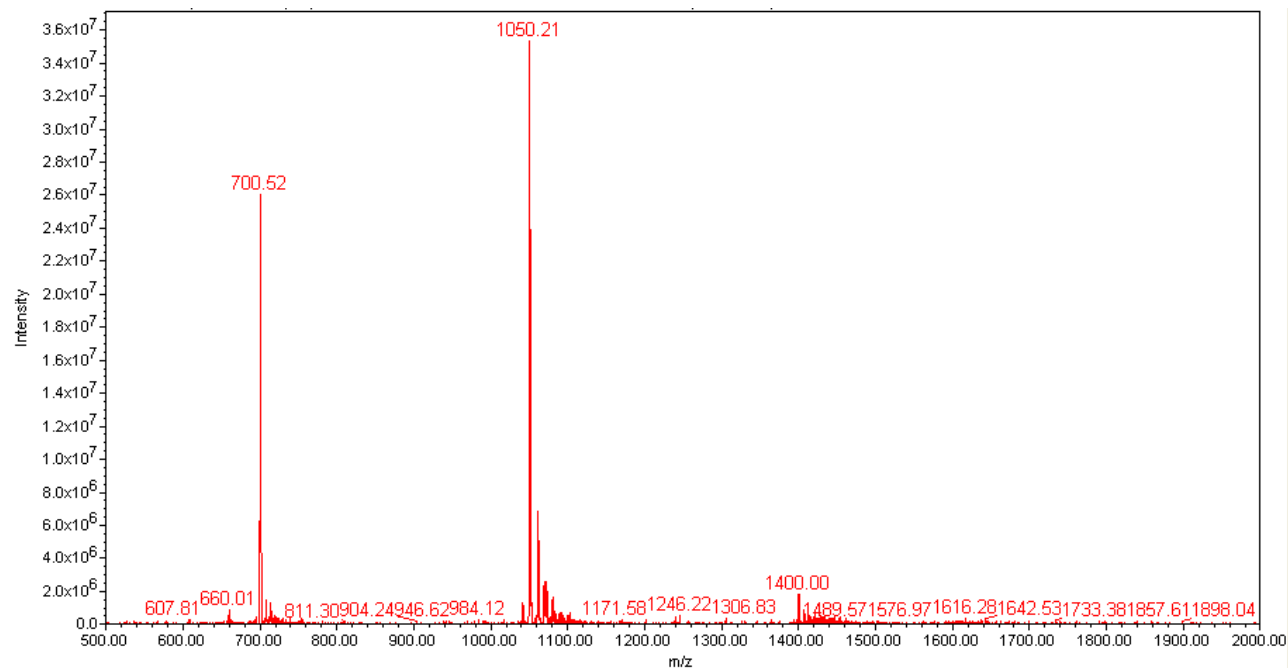

Figure S42. ESI-MS calcd. for $\mathrm{C}_{97} \mathrm{H}_{127} \mathrm{~N}_{21} \mathrm{O}_{32}, 2099.17$, $[\mathrm{M}+2 \mathrm{H}]^{2+} \mathrm{m} / \mathrm{z}=1050.59$, found $1050.21 ;[\mathrm{M}+3 \mathrm{H}]^{3+} \mathrm{m} / \mathrm{z}=700.73$, found 700.52 . 
Peptide 3f: ZIGGVGNYTLHAPTDS-CO-SAL ${ }^{\text {on }}$ ester

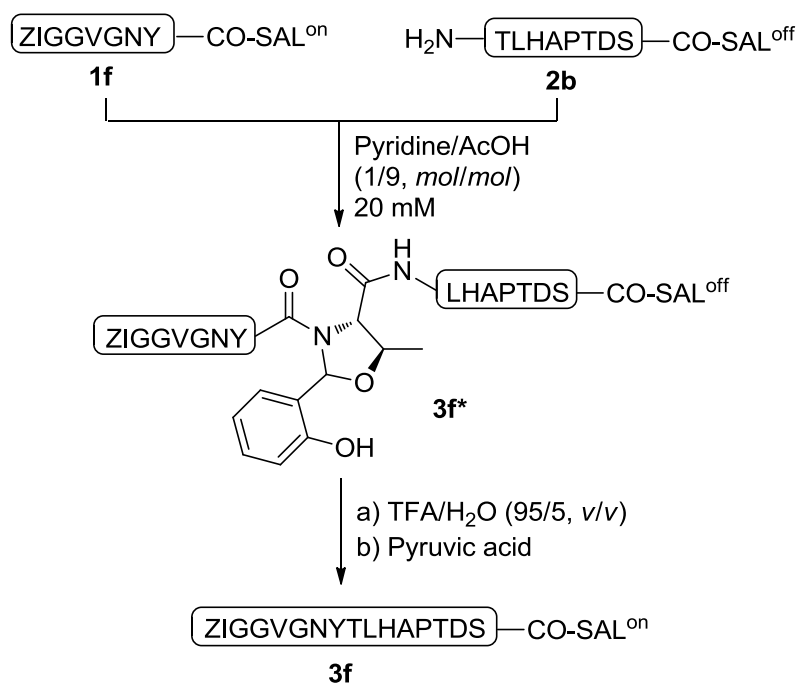

Scheme S6. Model N-to-C STL study between peptide $\mathbf{1 f}$ and $\mathbf{2 b}$.

Peptide $\mathbf{1 f}(5.0 \mathrm{mg}, 5.6 \mu \mathrm{mol})$ and peptide $\mathbf{2 b}(8.4 \mathrm{mg}, 8.4 \mu \mathrm{mol})$ were dissolved in pyridine/acetic acid $(1 / 9, \mathrm{~mol} / \mathrm{mol})$ buffer at a concentration of $20 \mathrm{mM}$ at room temperature. The reaction mixture was stirred at room temperature for $5 \mathrm{~h}$. After completion of the reaction, the solvent was blown off under a stream of condensed air. The residue was then treated with $1.0 \mathrm{~mL}$ of $\mathrm{TFA} / \mathrm{H}_{2} \mathrm{O}(95 / 5, v / v)$ and pyruvic acid (3.9 $\mu \mathrm{L}, 55.7 \mu \mathrm{mol})$. Preparative HPLC purification $\left(10-50 \% \mathrm{CH}_{3} \mathrm{CN} / \mathrm{H}_{2} \mathrm{O}\right.$ over 30 min) followed by concentration at reduced pressure and lyophilization afforded 4.5 $\mathrm{mg}$ (47\% yield) of peptide $\mathbf{3 f}$ as a white powder. 

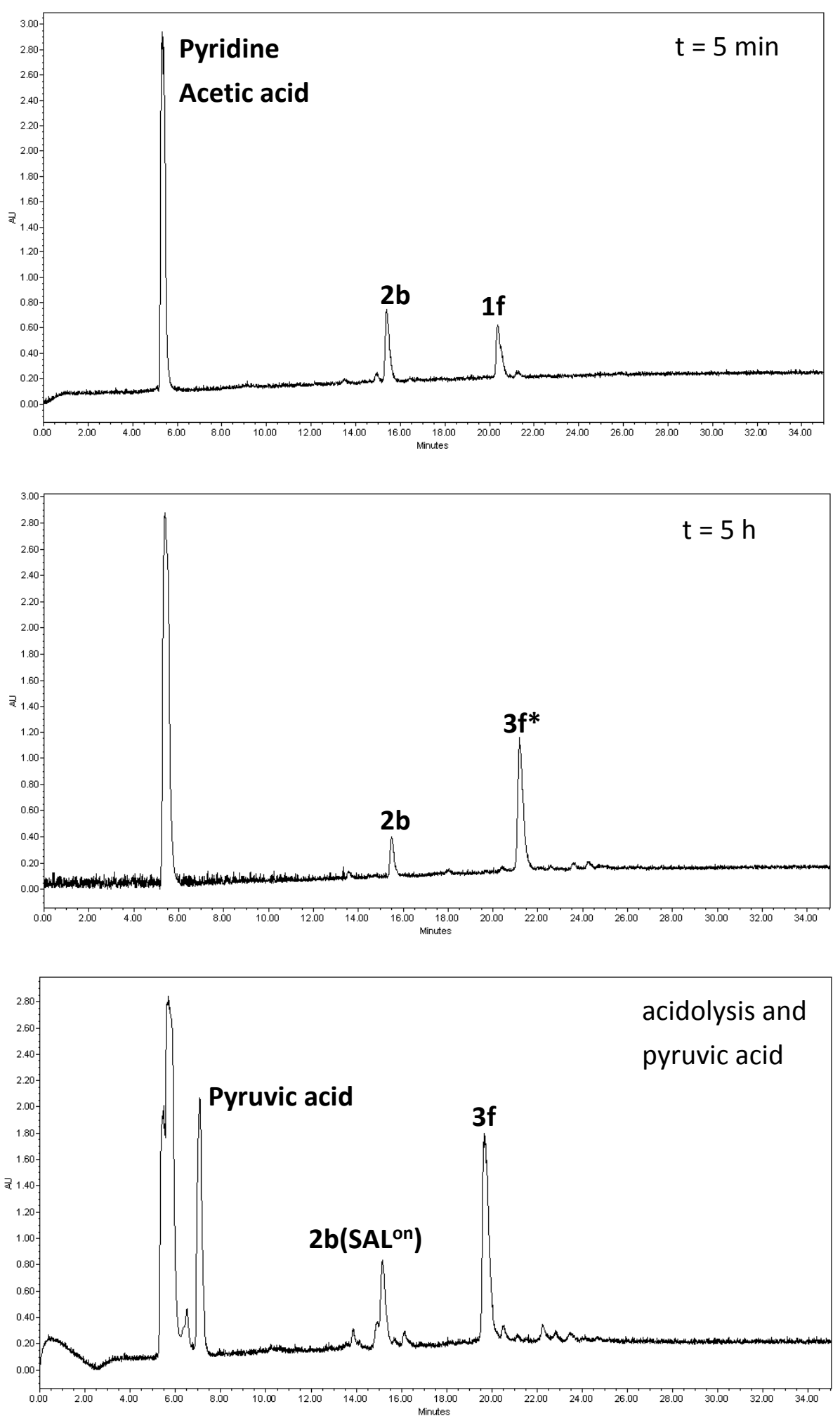

Figure S43. UV trace (190-400 $\mathrm{nm}$ ) from LC-MS analysis of STL between $\mathbf{1 f}$ and $\mathbf{2 b}$ at $5 \mathrm{~min}, 5 \mathrm{~h}$ to generate $\mathbf{3 f *}$ and after in situ acidolysis and pyruvic acid to give $\mathbf{3 f}$ : gradient $10-50 \% \mathrm{CH}_{3} \mathrm{CN} / \mathrm{H}_{2} \mathrm{O}$ containing $0.1 \%$ TFA over $30 \mathrm{~min}$ at a flow rate of 0.6 $\mathrm{mL} / \mathrm{min}$. 


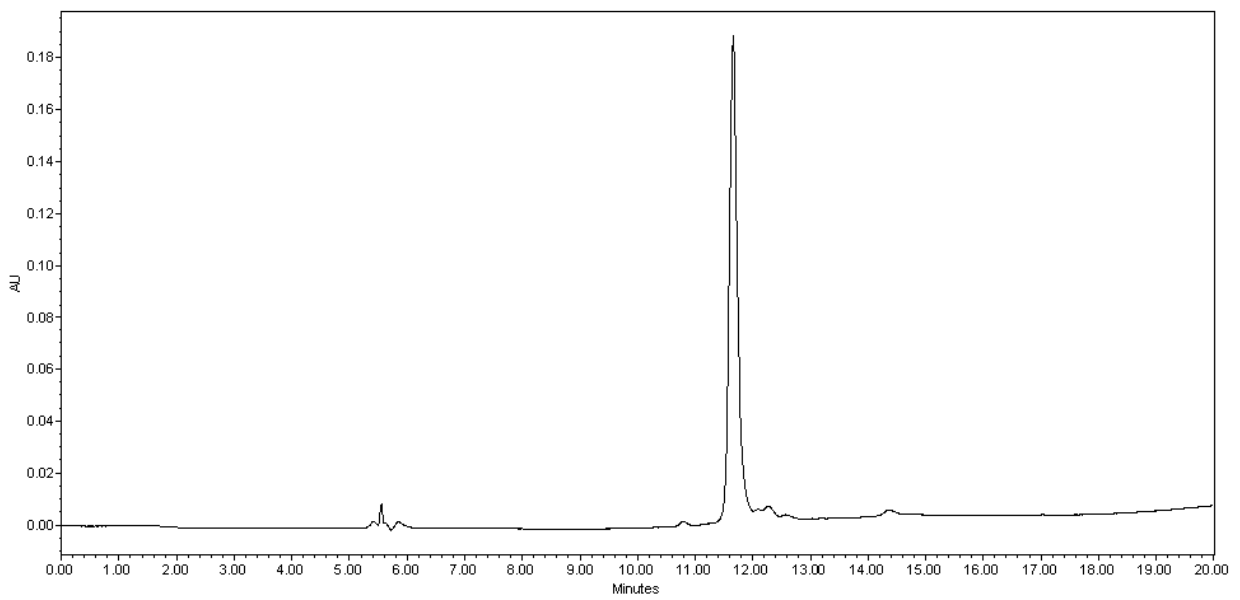

Figure S44. UV trace from analytical LC-MS analysis of purified peptide 3f: gradient $5-95 \% \mathrm{CH}_{3} \mathrm{CN} / \mathrm{H}_{2} \mathrm{O}$ containing $0.1 \%$ TFA over $15 \mathrm{~min}$ at a flow rate of $0.6 \mathrm{~mL} / \mathrm{min}$.

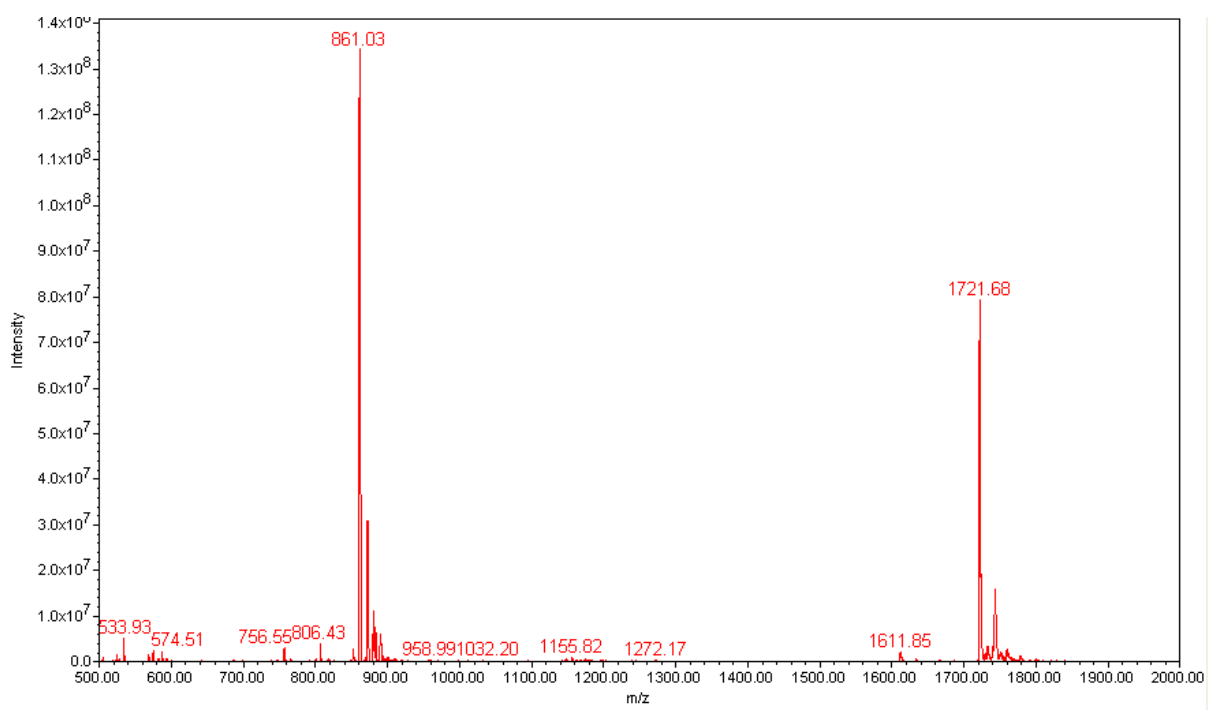

Figure S45. ESI-MS calcd. for $\mathrm{C}_{76} \mathrm{H}_{110} \mathrm{~N}_{19} \mathrm{O}_{25} \mathrm{~S}[\mathrm{M}+\mathrm{H}]^{+} m / z=1721.86$, found 1721.68; $[\mathrm{M}+2 \mathrm{H}]^{2+} \mathrm{m} / \mathrm{z}=861.44$, found 861.03. 


\section{Synthesis of peptide 5,7 and 8}

\section{Peptide 5: $\mathrm{H}_{2} \mathrm{~N}$-SGKVA-COOH}

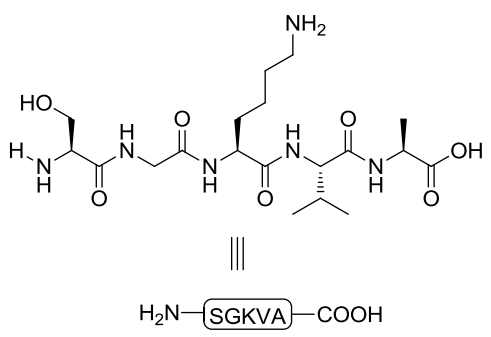

Peptide 5 was synthesized on 2-chlorotrityl chloride resin according to the general SPPS procedure A. The cleavage and global deprotection mixture was a mixture of TFA/ $H_{2} \mathrm{O} / \mathrm{TIPS}(95 / 2.5 / 2.5, v / v / v)$. Preparative HPLC purification $(0-50 \%$ $\mathrm{CH}_{3} \mathrm{CN} / \mathrm{H}_{2} \mathrm{O}$ over $30 \mathrm{~min}$ ) followed by concentration at reduced pressure and lyophilization afforded peptide $\mathbf{5}$ as a white powder.

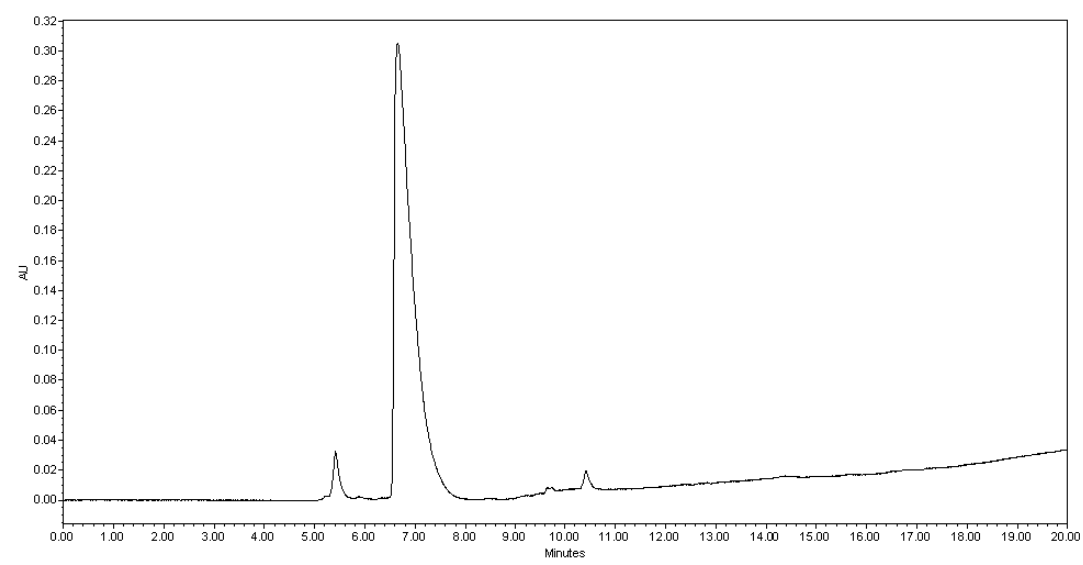

Figure S46. UV trace from analytical LC-MS analysis of purified peptide 5: gradient $5-95 \% \mathrm{CH}_{3} \mathrm{CN} / \mathrm{H}_{2} \mathrm{O}$ containing $0.1 \%$ TFA over $15 \mathrm{~min}$ at a flow rate of $0.6 \mathrm{~mL} / \mathrm{min}$. 


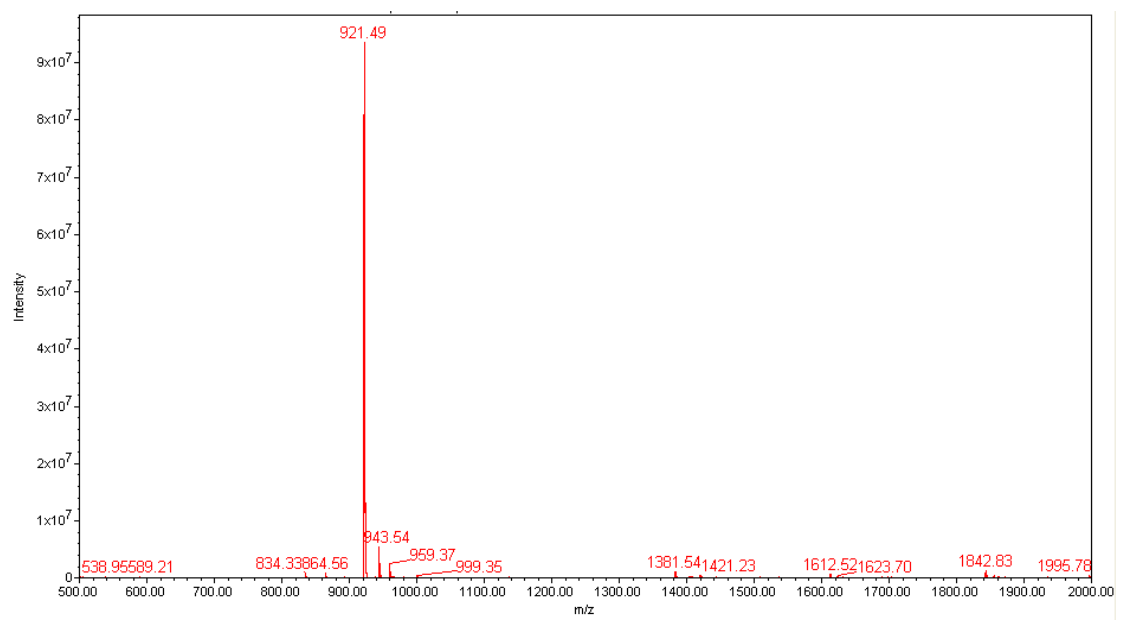

Figure S47. ESI-MS calcd. for $\mathrm{C}_{38} \mathrm{H}_{73} \mathrm{~N}_{12} \mathrm{O}_{14}[2 \mathrm{M}+\mathrm{H}]^{+} m / z=921.54$, found 921.49; $[2 \mathrm{M}+\mathrm{Na}]^{+} \mathrm{m} / z=943.51$, found 943.54 .

\section{Peptide 7: $\mathrm{H}_{2} \mathrm{~N}-\mathrm{SHKGY}-\mathrm{CO}-\mathrm{SPh}{ }^{1}$}

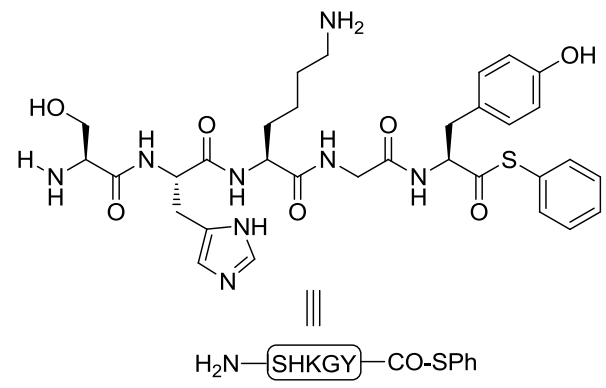

Crude protected peptide $\mathrm{BocHN}-\mathrm{Ser}\left({ }^{t} \mathrm{Bu}\right)-\mathrm{His}(\mathrm{Trt})-\mathrm{Lys}(\mathrm{Boc})-\mathrm{Gly}-\mathrm{COOH}$ (prepared according to general experimental procedure A and B) $(102.3 \mathrm{mg}, 110.6 \mu \mathrm{mol})$ was dissolved in $\mathrm{CHCl}_{3} /$ trifluoroethanol $(7.4 \mathrm{~mL})$, and $\mathrm{EDC}(51.4 \mathrm{mg}, 331.8 \mu \mathrm{mol})$ and HOOBt $(54.1 \mathrm{mg}, 331.8 \mu \mathrm{mol})$ were then added. After $5 \mathrm{~min}, \mathbf{H C l} \cdot \boldsymbol{H}_{2} \boldsymbol{N}$-Tyr-SPh (102.5 mg, $331.8 \mu \mathrm{mol}$ ) was added, and the reaction mixture was stirred for $3 \mathrm{~h}$ to form the crude protected C-terminal peptide thioester. The reaction mixture was then concentrated and subjected to $7.0 \mathrm{~mL}$ of TFA/ $\mathrm{H}_{2} \mathrm{O} / \mathrm{TIPS}(95 / 2.5 / 2.5, v / v / v)$ for $1 \mathrm{~h}$. Preparative HPLC purification $\left(10-40 \% \mathrm{CH}_{3} \mathrm{CN} / \mathrm{H}_{2} \mathrm{O}\right.$ over $\left.30 \mathrm{~min}\right)$ followed by concentration at reduced pressure and lyophilization afforded peptide 7 (24.1 $\mathrm{mg}$, $32 \%$ yield) as a white powder. 


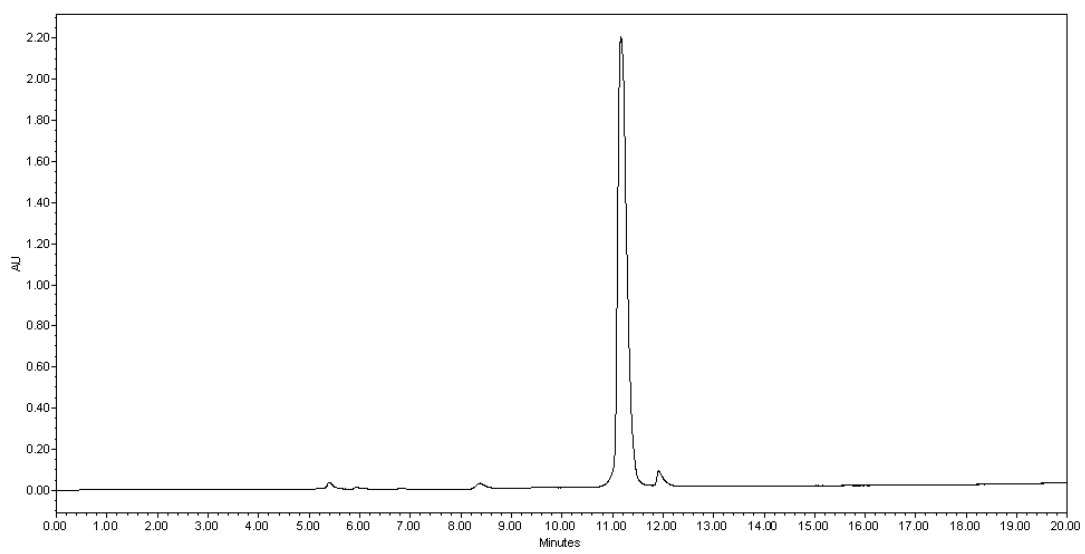

Figure S48. UV trace from analytical LC-MS analysis of purified peptide 5: gradient $5-95 \% \mathrm{CH}_{3} \mathrm{CN} / \mathrm{H}_{2} \mathrm{O}$ containing $0.1 \% \mathrm{TFA}$ over $15 \mathrm{~min}$ at a flow rate of $0.6 \mathrm{~mL} / \mathrm{min}$.

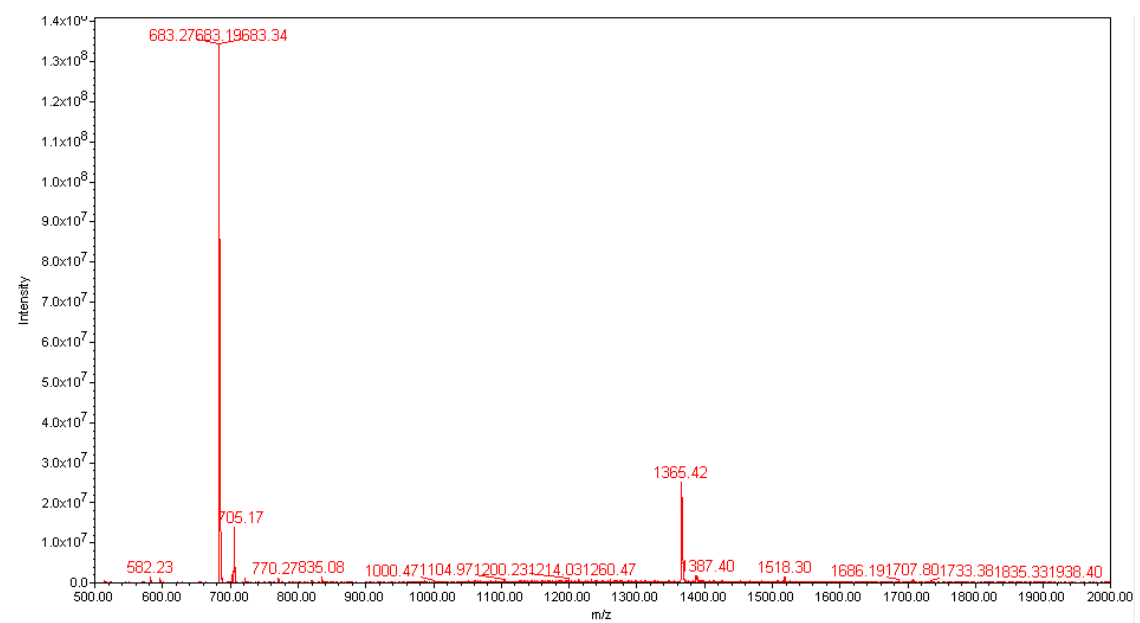

Figure S49. ESI-MS calcd. for $\mathrm{C}_{32} \mathrm{H}_{43} \mathrm{~N}_{8} \mathrm{O}_{7} \mathrm{~S}[\mathrm{M}+\mathrm{H}]^{+} \mathrm{m} / z=683.29$, found 683.34; $[2 \mathrm{M}+\mathrm{H}]^{+} m / z=1365.59$, found 1365.42 .

\section{Peptide 8: $\mathrm{H}_{2} \mathrm{~N}$-CKEPVHGV-COOH}

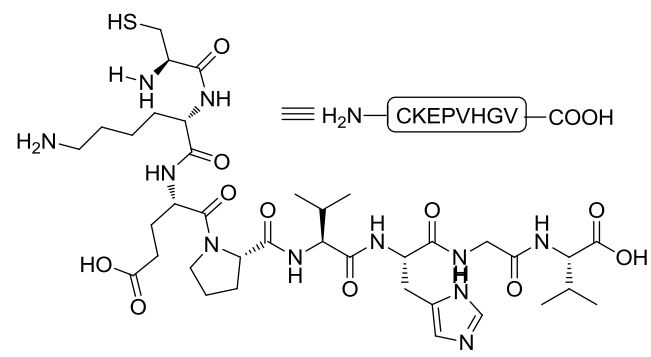

Peptide 8 was synthesized on 2-chlorotrityl chloride resin according to the general SPPS procedure A. The cleavage and global deprotection mixture was a mixture of 
TFA/ $\mathrm{H}_{2} \mathrm{O} / \mathrm{TIPS} \quad(95 / 2.5 / 2.5, v / v / v)$. Preparative HPLC purification $(10-50 \%$ $\mathrm{CH}_{3} \mathrm{CN} / \mathrm{H}_{2} \mathrm{O}$ over $30 \mathrm{~min}$ ) followed by concentration at reduced pressure and lyophilization afforded peptide $\mathbf{8}$ as a white powder.

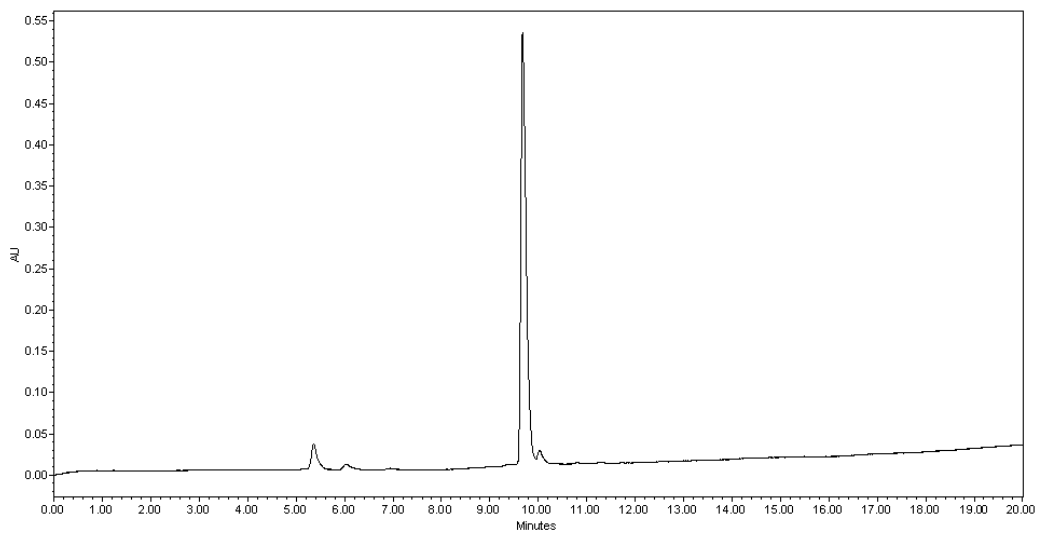

Figure S50. UV trace from analytical LC-MS analysis of purified peptide 8: gradient $5-95 \% \mathrm{CH}_{3} \mathrm{CN} / \mathrm{H}_{2} \mathrm{O}$ containing $0.1 \%$ TFA over $15 \mathrm{~min}$ at a flow rate of $0.6 \mathrm{~mL} / \mathrm{min}$.

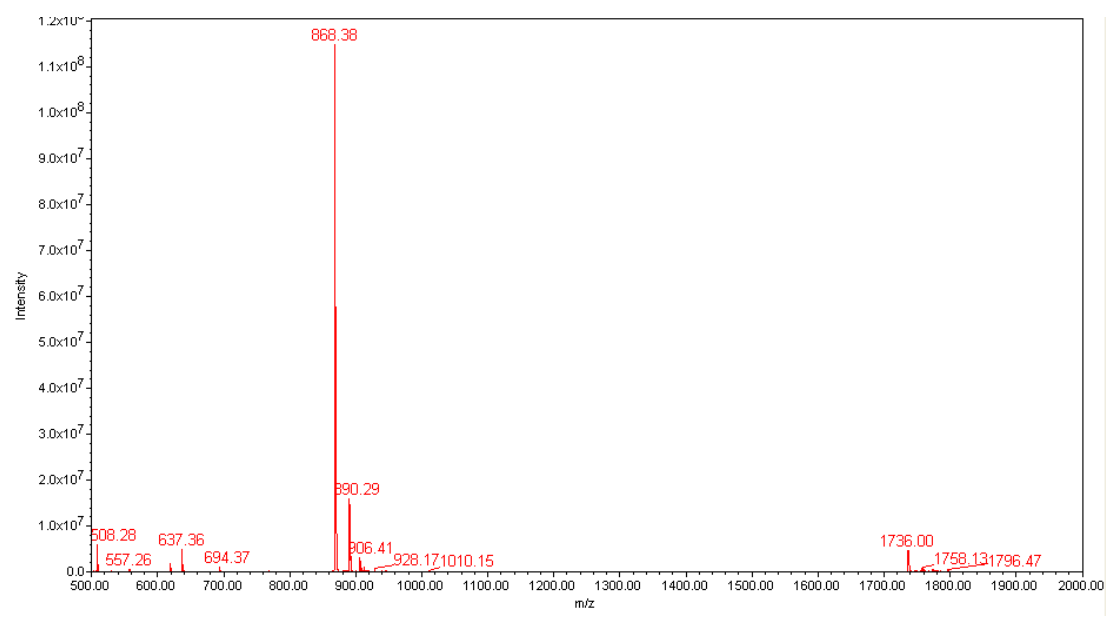

Figure S51. ESI-MS calcd. for $\mathrm{C}_{37} \mathrm{H}_{62} \mathrm{~N}_{11} \mathrm{O}_{11} \mathrm{~S}[\mathrm{M}+\mathrm{H}]^{+} m / z=868.43$, found 868.38; $[\mathrm{M}+\mathrm{Na}]^{+} \mathrm{m} / z=890.41$, found $890.29 ;[2 \mathrm{M}+\mathrm{H}]^{+} \mathrm{m} / z=1735.86$, found 1736.00 . 


\section{Application of $N$-to- $C$ strategy on convergent STL}

\section{A. Synthesis of a model C-terminal peptide SAL ${ }^{\text {on }}$ ester to demonstrate the feasibility on tandem STL in the $N$-to- $C$ direction}

Peptide 4a: $\mathrm{H}_{2} \mathrm{~N}$-VIGGVGNATLHAPTDYTLHAPTDA- $\mathrm{CO}-\mathrm{SAL}^{\text {on }}$ ester

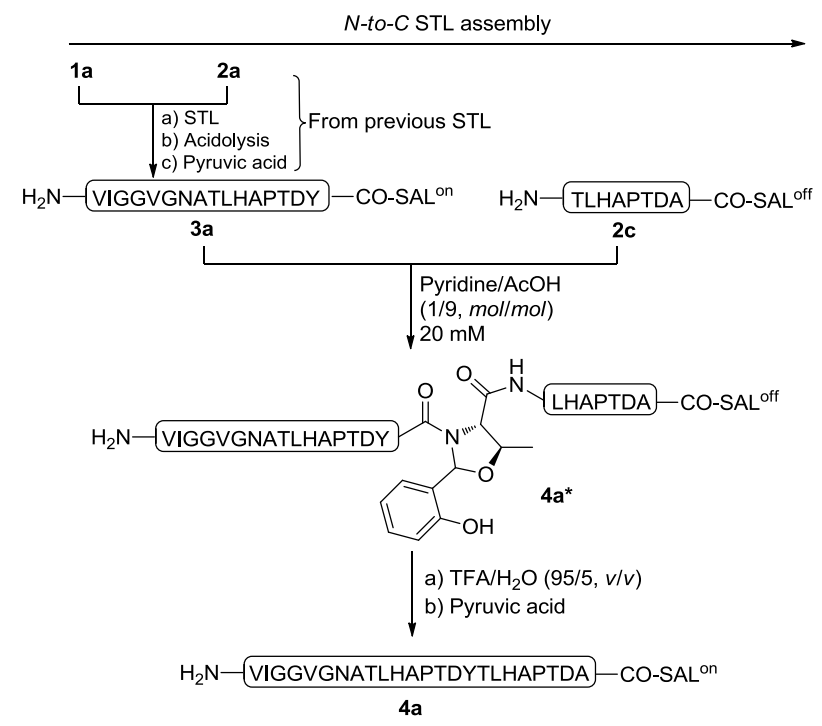

Scheme S7. Model N-to-C STL study between peptide 3a and 2c.

Peptide 3a (3.0 mg, $1.8 \mu \mathrm{mol}$ ) (obtained previously from $\mathrm{N}$-to- $\mathrm{C}$ ligation using $1 \mathbf{a}$ and 2a) and peptide $2 \mathbf{c}(1.6 \mathrm{mg}, 1.8 \mu \mathrm{mol})$ were dissolved in pyridine/acetic acid (1/9, $\mathrm{mol} / \mathrm{mol}$ ) buffer at a concentration of $20 \mathrm{mM}$ at room temperature. The reaction mixture was stirred at room temperature for $4 \mathrm{~h}$. After completion of the reaction, the solvent was blown off under a stream of condensed air. The residue was then treated with $1.0 \mathrm{~mL}$ of $\mathrm{TFA} / \mathrm{H}_{2} \mathrm{O}(95 / 5, v / v)$ and pyruvic acid $(1.3 \mu \mathrm{L}, 17.8 \mu \mathrm{mol})$. Preparative HPLC purification $\left(10-50 \% \quad \mathrm{CH}_{3} \mathrm{CN} / \mathrm{H}_{2} \mathrm{O}\right.$ over $30 \mathrm{~min}$ ) followed by concentration at reduced pressure and lyophilization afforded $2.3 \mathrm{mg}$ (51\% yield) of peptide $\mathbf{3 f}$ as a white powder. 

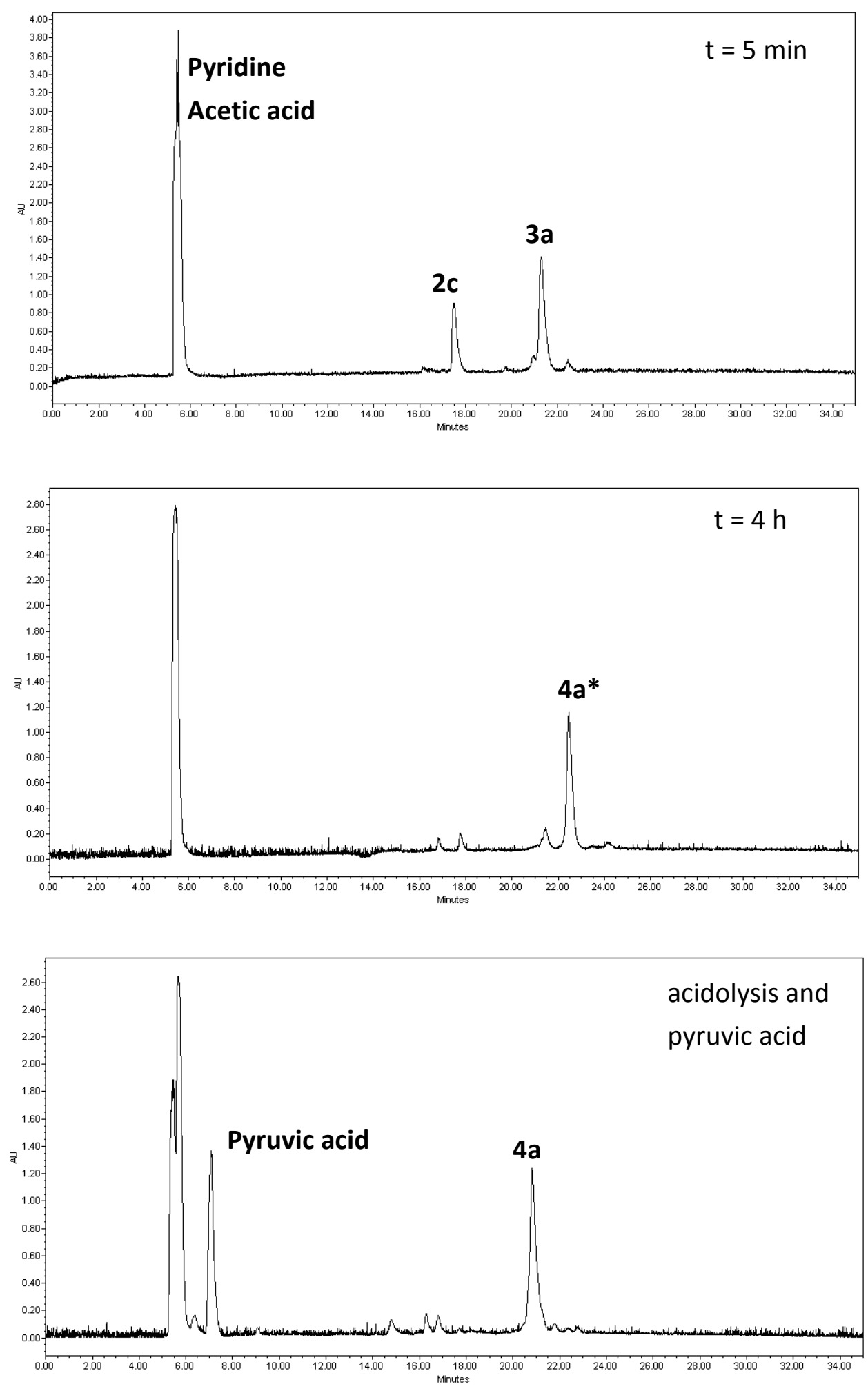

Figure S52. UV trace (190-400 nm) from LC-MS analysis of STL between 3a and 2c at $5 \mathrm{~min}, 4 \mathrm{~h}$ to generate $\mathbf{4 a} *$ and after in situ acidolysis and pyruvic acid to give $\mathbf{4 a}$ : gradient $10-50 \% \mathrm{CH}_{3} \mathrm{CN} / \mathrm{H}_{2} \mathrm{O}$ containing $0.1 \%$ TFA over $30 \mathrm{~min}$ at a flow rate of 0.6 $\mathrm{mL} / \mathrm{min}$. 


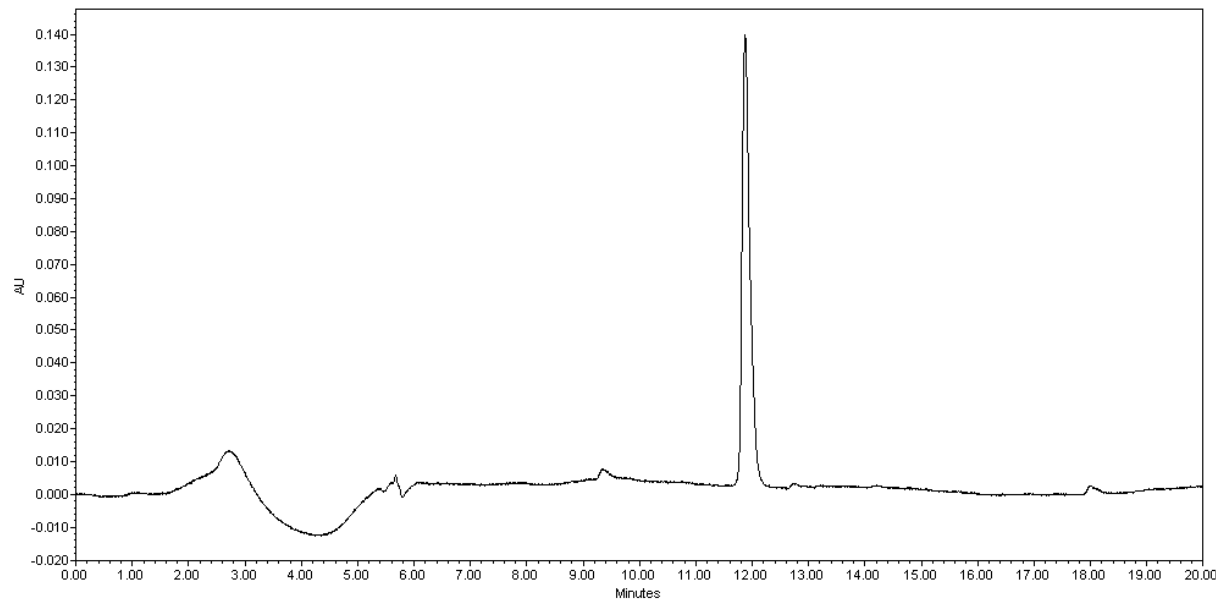

Figure S53. UV trace from analytical LC-MS analysis of purified peptide 4a:

gradient 5-95\% $\mathrm{CH}_{3} \mathrm{CN} / \mathrm{H}_{2} \mathrm{O}$ containing $0.1 \%$ TFA over $15 \mathrm{~min}$ at a flow rate of 0.6 $\mathrm{mL} / \mathrm{min}$.

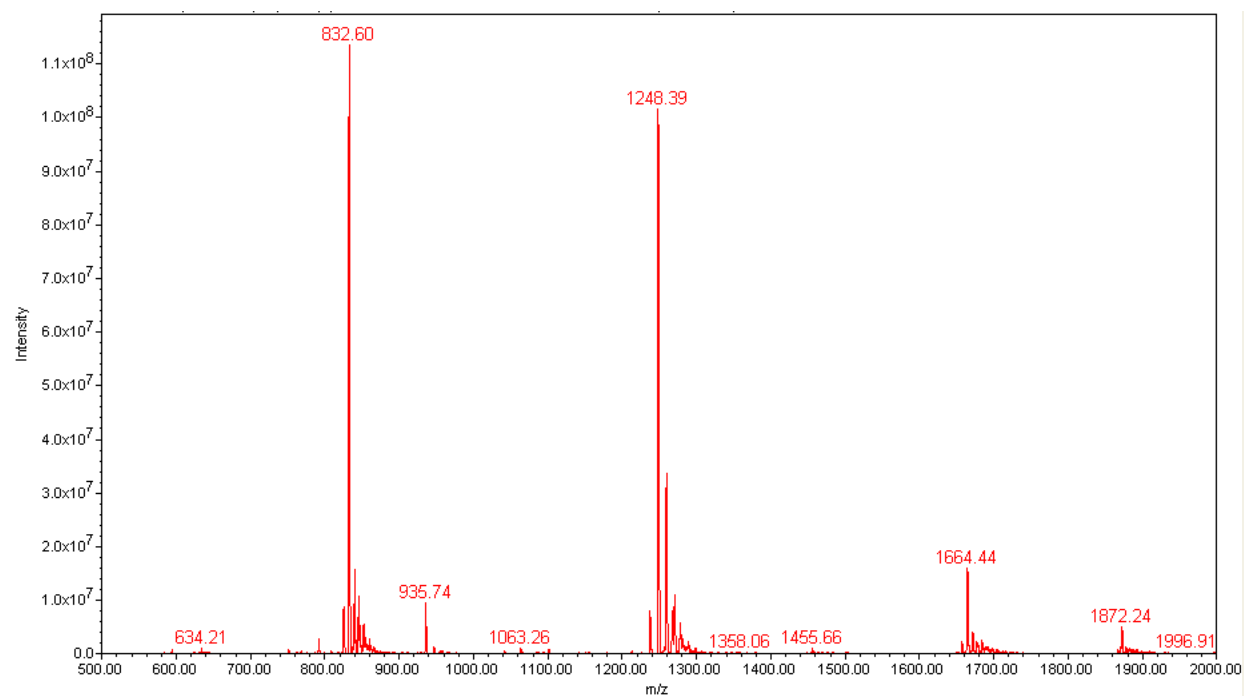

Figure S54. ESI-MS calcd. for $\mathrm{C}_{112} \mathrm{H}_{167} \mathrm{~N}_{29} \mathrm{O}_{36}, 2495.70,[2 \mathrm{M}+3 \mathrm{H}]^{3+} \mathrm{m} / \mathrm{z}=1664.81$, found 1664.44; $[\mathrm{M}+2 \mathrm{H}]^{2+} \mathrm{m} / \mathrm{z}=1248.86$, found 1248.39; $[\mathrm{M}+3 \mathrm{H}]^{3+} \mathrm{m} / \mathrm{z}=832.91$, found 832.60 . 
B. Synthesis of a model N-terminal Serine peptide using STL in novel $N$-to-C direction rather than traditional $C$-to- $N$ direction

Peptide 4b: $\mathrm{H}_{2} \mathrm{~N}$-SEHDKTAYTLHAPTDSSGKVA-COOH

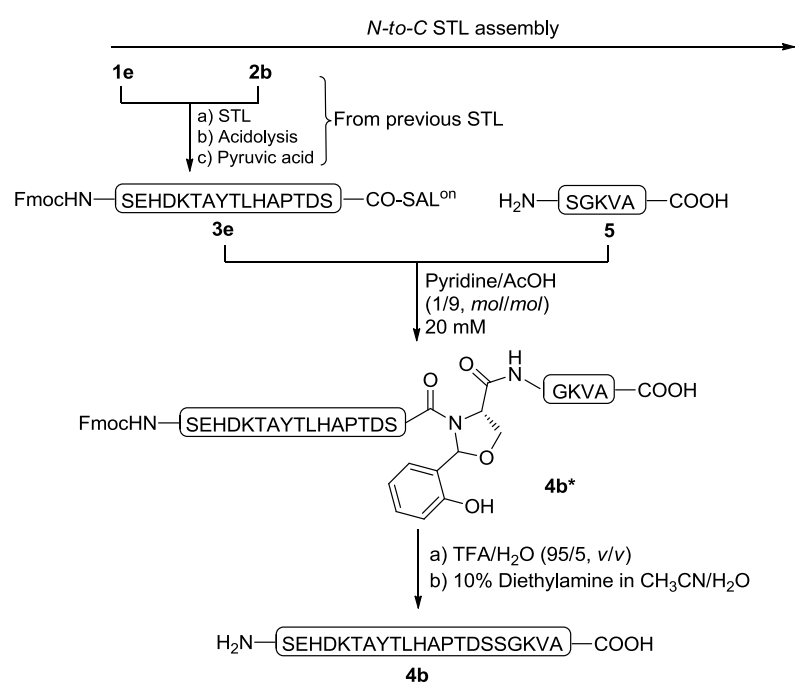

Scheme S8. Model N-to-C STL study between peptide $\mathbf{3 e}$ and $\mathbf{5}$.

Peptide 3e (3.0 mg, $1.4 \mu \mathrm{mol})$ (obtained previously from $\mathrm{N}$-to-C ligation using $1 \mathbf{e}$ and 2b) and peptide $5(1.3 \mathrm{mg}, 2.9 \mu \mathrm{mol})$ were dissolved in pyridine/acetic acid (1/9, $\mathrm{mol} / \mathrm{mol}$ ) buffer at a concentration of $20 \mathrm{mM}$ at room temperature. The reaction mixture was stirred at room temperature for $3 \mathrm{~h}$. After completion of the reaction, the solvent was blown off under a stream of condensed air. The residue was then treated with $1.0 \mathrm{~mL}$ of $\mathrm{TFA} / \mathrm{H}_{2} \mathrm{O} \quad(95 / 5, v / v)$ and followed by $0.5 \mathrm{~mL}$ of diethylamine/ $\mathrm{CH}_{3} \mathrm{CN} / \mathrm{H}_{2} \mathrm{O}(1 / 4.5 / 4.5, v / v / v)$ for $1 \mathrm{~h}$ at room temperature to remove terminal Fmoc protecting group. Preparative HPLC purification (10-50\% $\mathrm{CH}_{3} \mathrm{CN} / \mathrm{H}_{2} \mathrm{O}$ over $30 \mathrm{~min}$ ) followed by concentration at reduced pressure and lyophilization afforded $1.3 \mathrm{mg}$ (40\% yield) of peptide $\mathbf{4 b}$ as a white powder. 

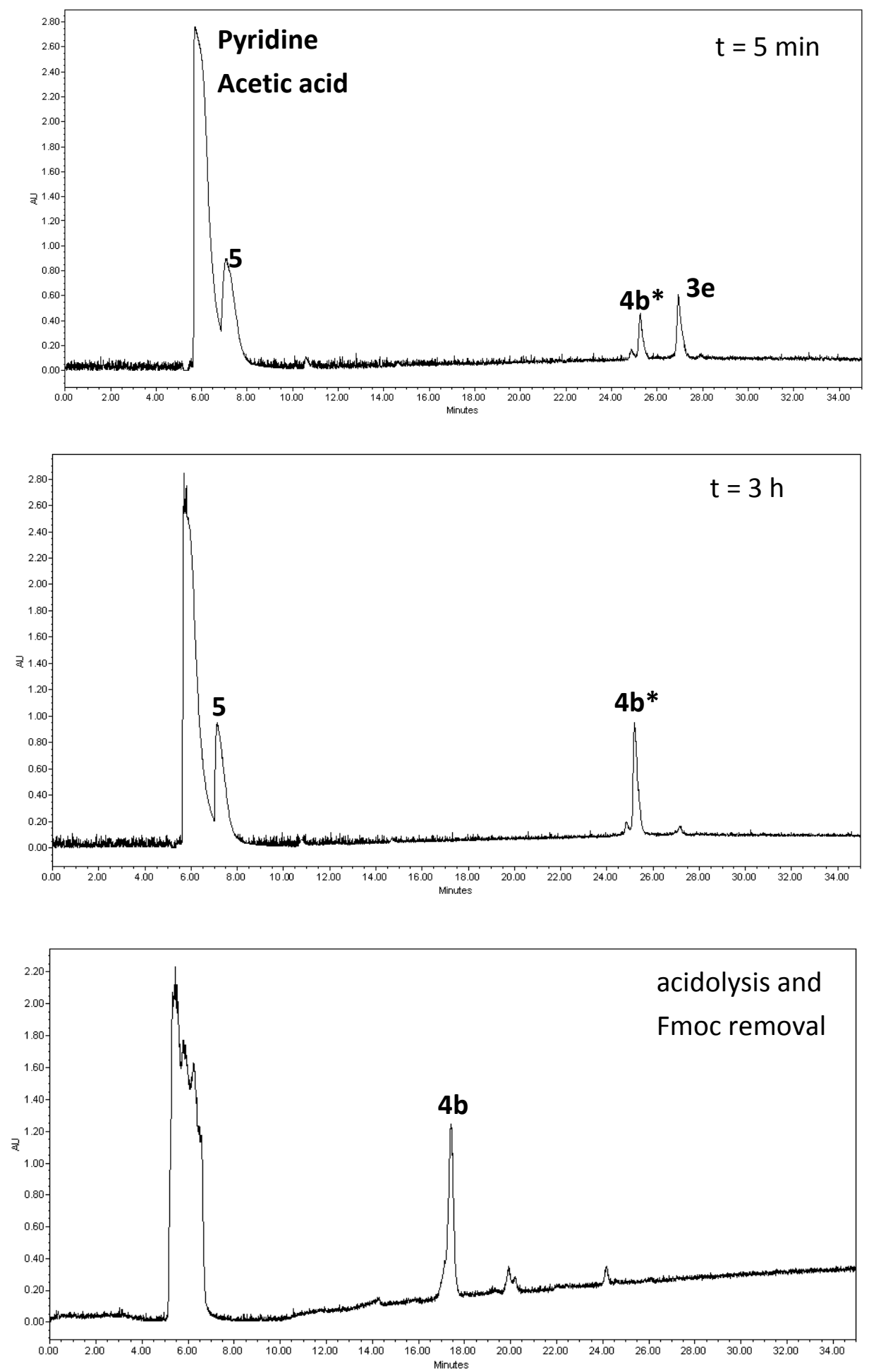

Figure S55. UV trace (190-400 nm) from LC-MS analysis of STL between 3e and 5 at $5 \mathrm{~min}, 3 \mathrm{~h}$ to generate $\mathbf{4 b} *$ and after acidolysis and Fmoc removal to give $\mathbf{4 b}$ : gradient $0-50 \% \mathrm{CH}_{3} \mathrm{CN} / \mathrm{H}_{2} \mathrm{O}$ containing $0.1 \%$ TFA over $30 \mathrm{~min}$ at a flow rate of 0.6 $\mathrm{mL} / \mathrm{min}$. 


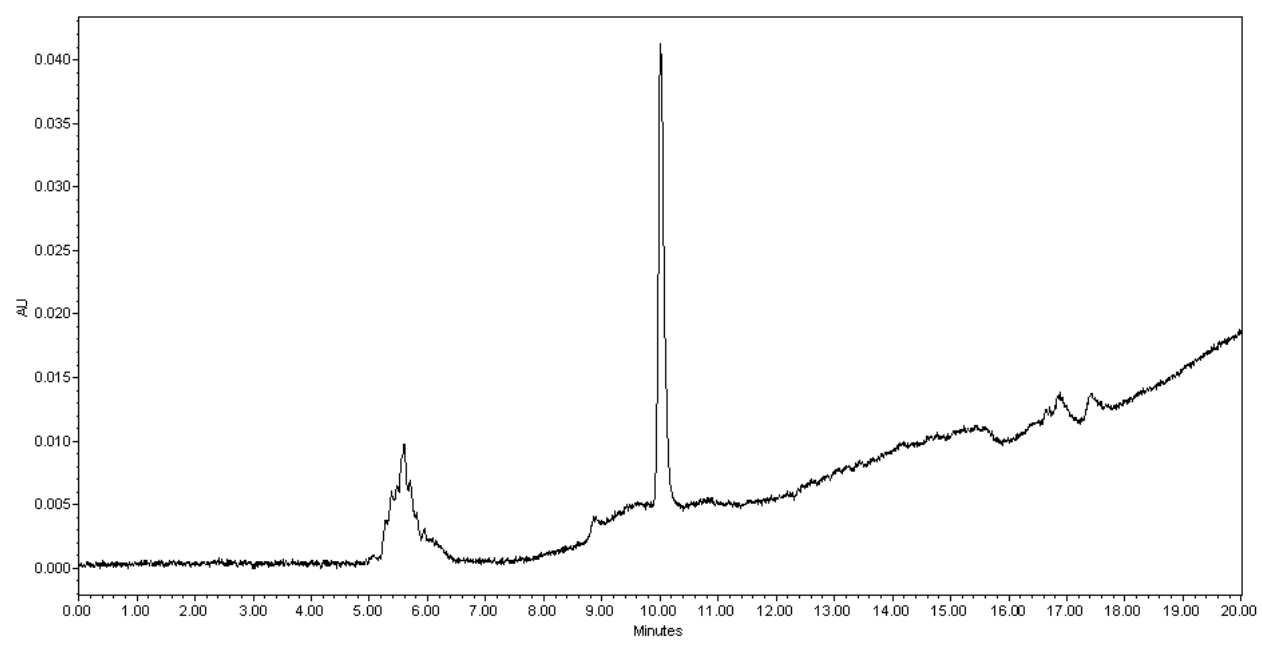

Figure S56. UV trace from analytical LC-MS analysis of purified peptide $\mathbf{4 b}$ : gradient 5-95\% $\mathrm{CH}_{3} \mathrm{CN} / \mathrm{H}_{2} \mathrm{O}$ containing $0.1 \%$ TFA over $15 \mathrm{~min}$ at a flow rate of 0.6 $\mathrm{mL} / \mathrm{min}$.

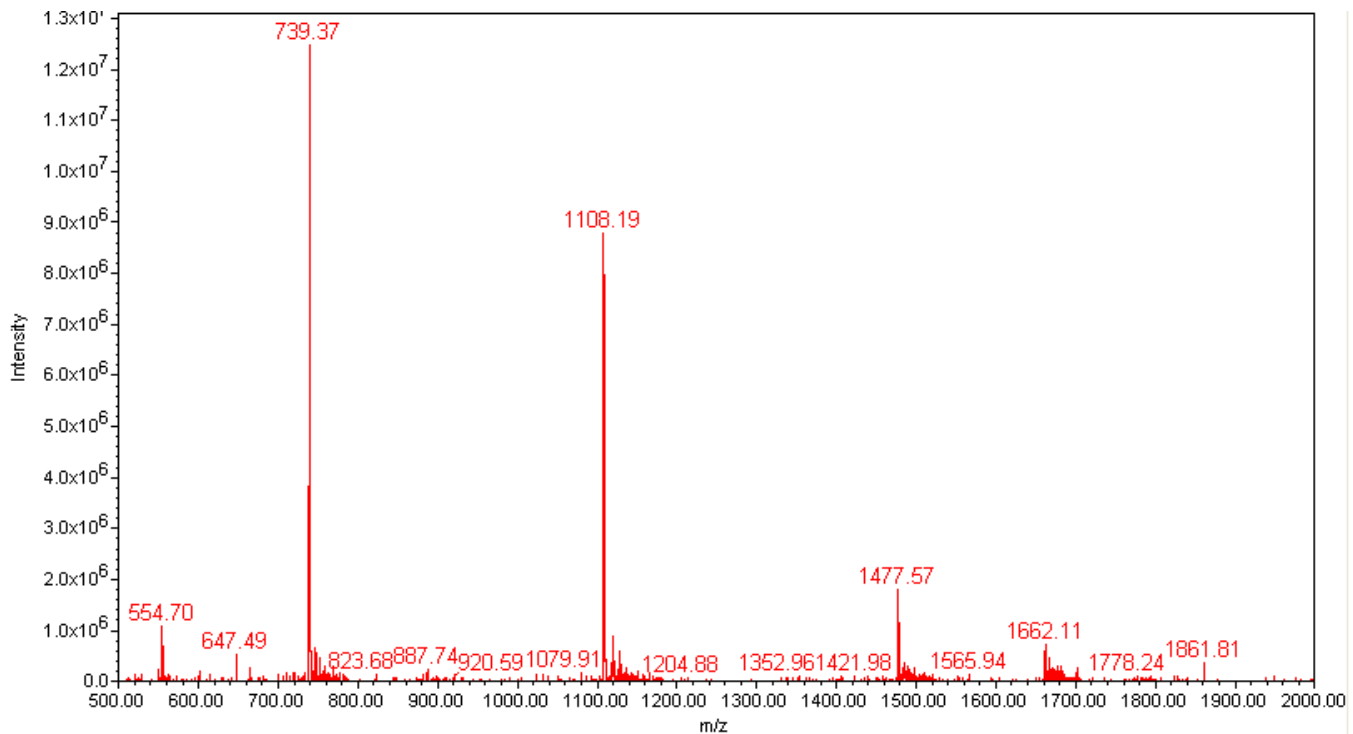

Figure S57. ESI-MS calcd. for $\mathrm{C}_{94} \mathrm{H}_{147} \mathrm{~N}_{27} \mathrm{O}_{35}, 2215.33$, $[2 \mathrm{M}+3 \mathrm{H}]^{3+} \mathrm{m} / z=1477.90$, found 1477.57; $[\mathrm{M}+2 \mathrm{H}]^{2+} \mathrm{m} / \mathrm{z}=1108.67$, found 1108.19; $[\mathrm{M}+3 \mathrm{H}]^{3+} \mathrm{m} / \mathrm{z}=739.45$, found 739.37 . 


\section{Realization of Convergent STL}

\section{Peptide 6:}

$\mathrm{H}_{2} \mathrm{~N}$-VIGGVGNATLHAPTDYTLHAPTDASEHDKTAYTLHAPTDSSGKVA-C $\mathrm{OOH}$

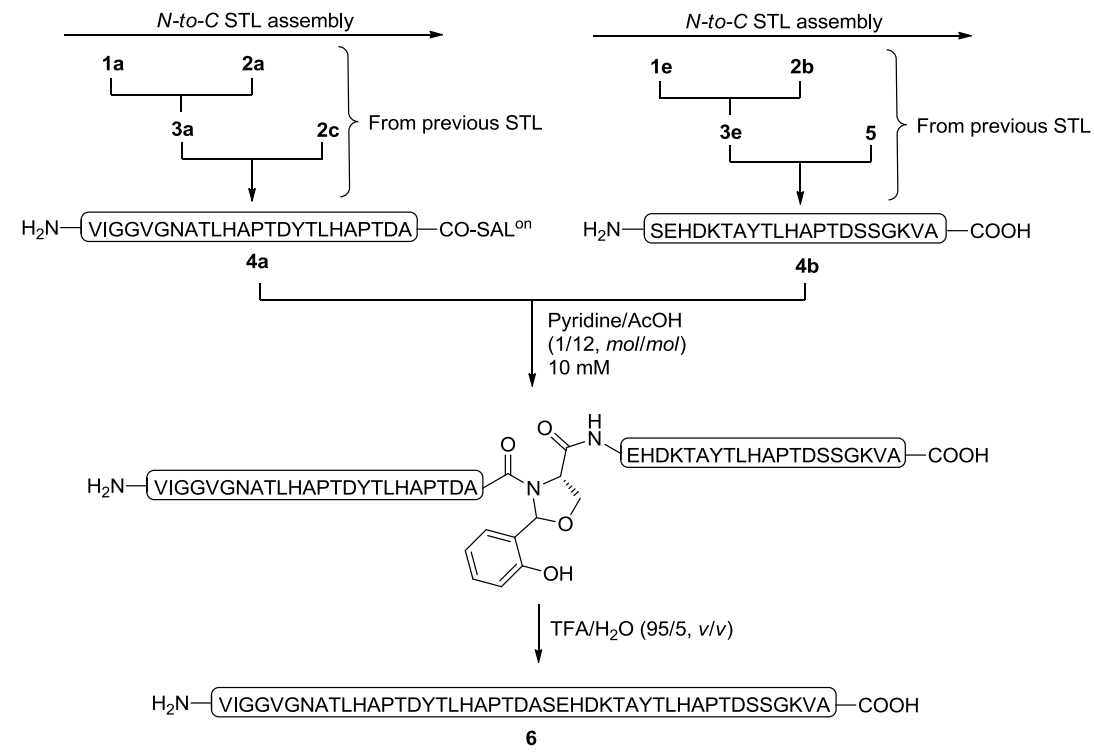

Scheme S9. Model $N$-to- $C$ STL study between peptide $\mathbf{4 a}$ and $\mathbf{4 b}$.

Peptide 4a (1.0 mg, $0.4 \mu \mathrm{mol})$ (obtained previously from $N$-to- $C$ ligation using 3a and 2c) and peptide $4 \mathbf{b}(1.3 \mathrm{mg}, 0.6 \mu \mathrm{mol})$ (obtained previously from $N$-to- $C$ ligation using 3e and 5) were dissolved in pyridine/acetic acid $(1 / 12, \mathrm{~mol} / \mathrm{mol})$ buffer at a concentration of $10 \mathrm{mM}$ at room temperature. The reaction mixture was stirred at room temperature for $6 \mathrm{~h}$. After completion of the reaction, the solvent was blown off under a stream of condensed air. The residue was then treated with $1.0 \mathrm{~mL}$ of TFA/ $\mathrm{H}_{2} \mathrm{O}(95 / 5, v / v)$. Preparative HPLC purification $\left(10-50 \% \mathrm{CH}_{3} \mathrm{CN} / \mathrm{H}_{2} \mathrm{O}\right.$ over 30 min) followed by concentration at reduced pressure and lyophilization afforded 1.1 mg (59\% yield) of peptide 6 as a white powder. 

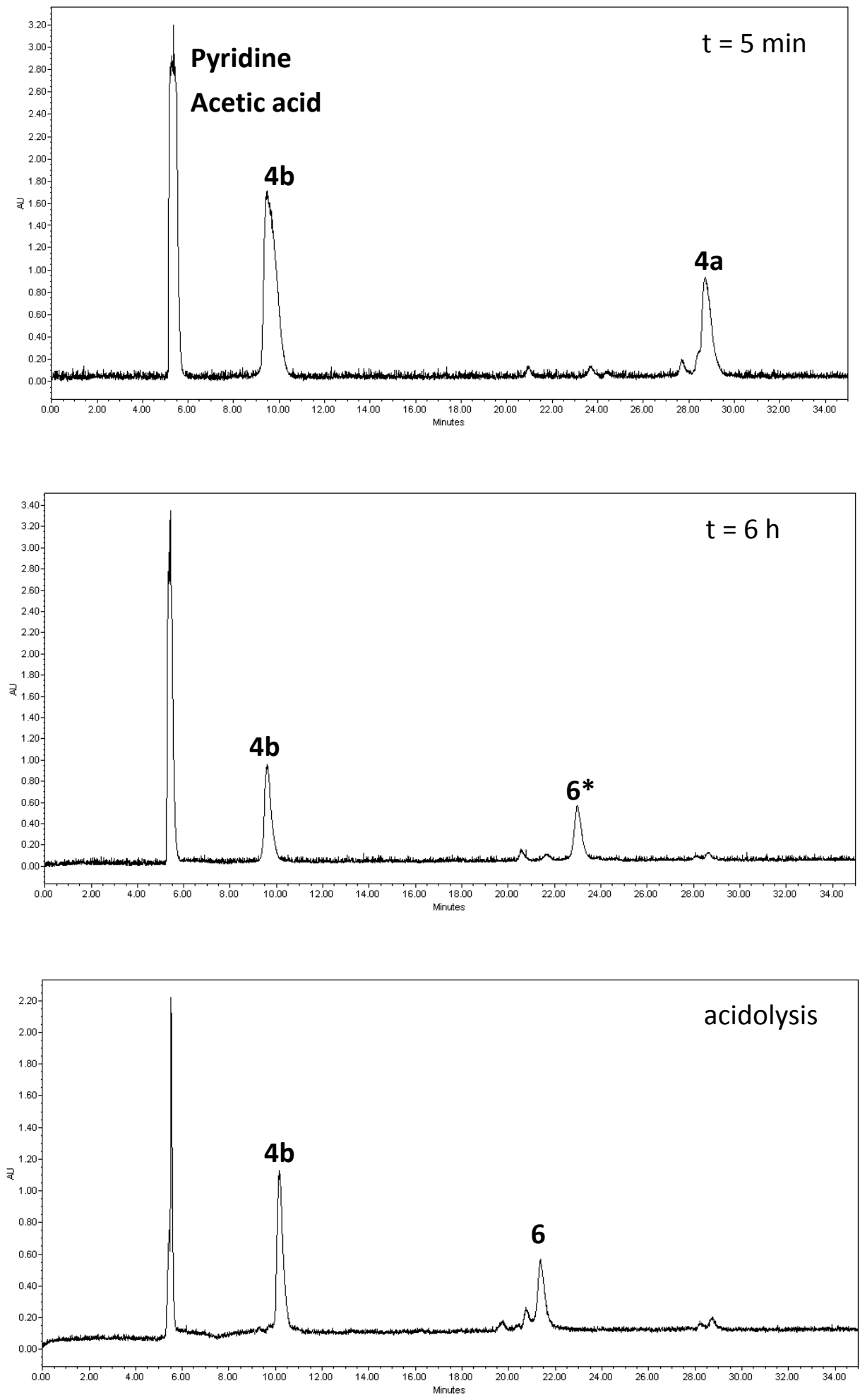

Figure S58. UV trace (190-400 nm) from LC-MS analysis of STL between $\mathbf{4 a}$ and $\mathbf{4 b}$ at $5 \mathrm{~min}, 6 \mathrm{~h}$ to generate $\mathbf{6}^{*}$ and after acidolysis to give 6: gradient $15-30 \%$ $\mathrm{CH}_{3} \mathrm{CN} / \mathrm{H}_{2} \mathrm{O}$ containing $0.1 \%$ TFA over $30 \mathrm{~min}$ at a flow rate of $0.6 \mathrm{~mL} / \mathrm{min}$. 


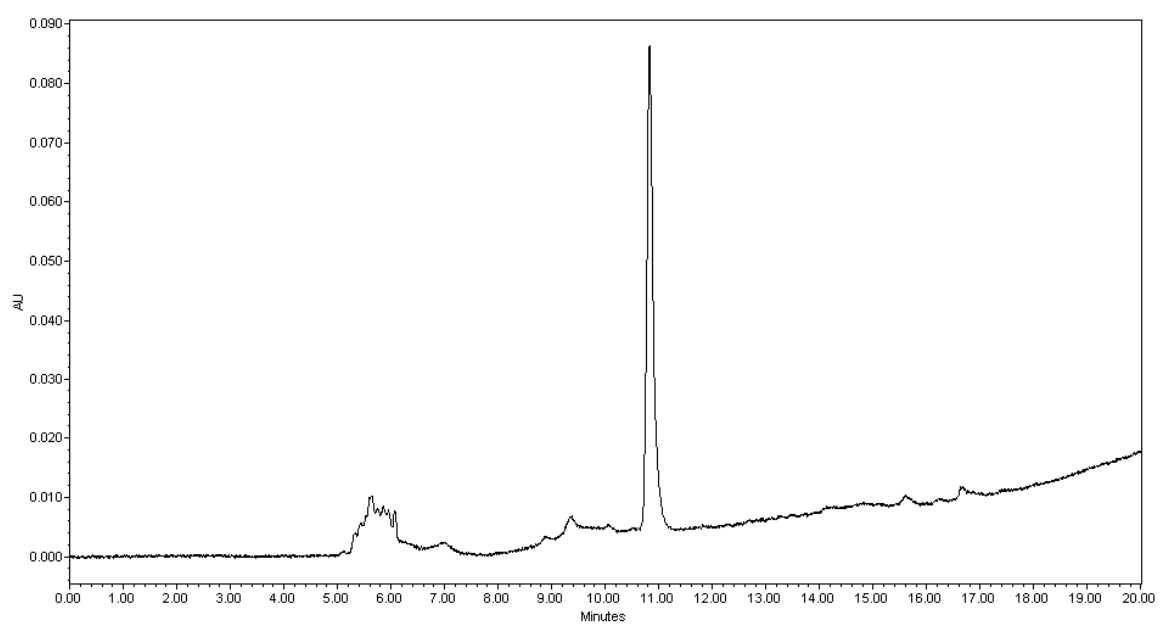

Figure S59. UV trace from analytical LC-MS analysis of purified peptide 6: gradient $5-95 \% \mathrm{CH}_{3} \mathrm{CN} / \mathrm{H}_{2} \mathrm{O}$ containing $0.1 \%$ TFA over $15 \mathrm{~min}$ at a flow rate of $0.6 \mathrm{~mL} / \mathrm{min}$.

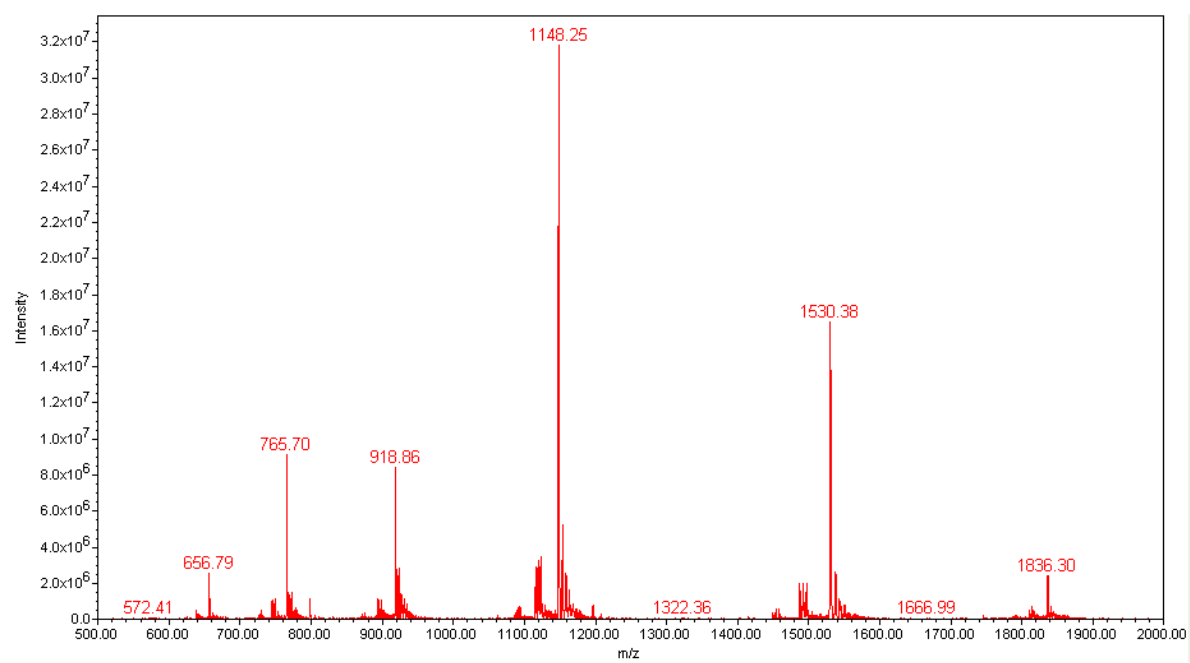

Figure S60. ESI-MS calcd. for $\mathrm{C}_{199} \mathrm{H}_{308} \mathrm{~N}_{56} \mathrm{O}_{69}, 4588.91,[2 \mathrm{M}+5 \mathrm{H}]^{5+} \mathrm{m} / z=1836.57$, found 1836.30; $[\mathrm{M}+3 \mathrm{H}]^{3+} \mathrm{m} / z=1530.64$, found 1530.38; $[\mathrm{M}+4 \mathrm{H}]^{4+} \mathrm{m} / z=1148.23$, found 1148.25; $[\mathrm{M}+5 \mathrm{H}]^{5+} \mathrm{m} / \mathrm{z}=918.79$, found 918.86; $[\mathrm{M}+6 \mathrm{H}]^{6+} \mathrm{m} / \mathrm{z}=765.83$, found 765.70; $[\mathrm{M}+7 \mathrm{H}]^{7+} \mathrm{m} / z=656.57$, found 656.79 . 


\section{One-pot Three-segment STL and NCL}

\section{Peptide 9: $\mathrm{H}_{2} \mathrm{~N}$-CIGGVGNYTLHAPTDSSHKGYCKEPVHGV-COOH}

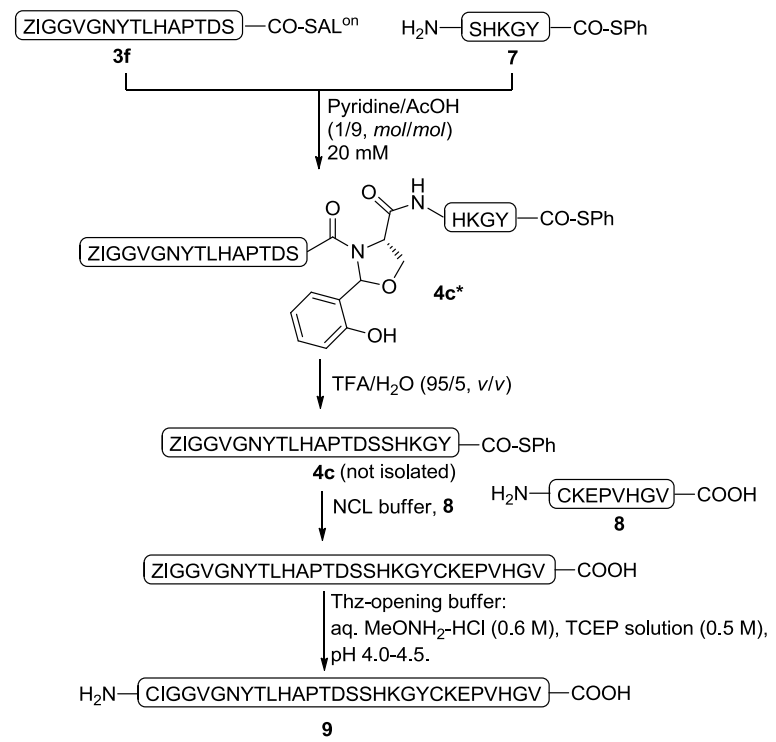

Scheme S10. One-pot Three-segment STL and NCL study between peptide 3f, 7 and 8.

Peptide 3f $(3.0 \mathrm{mg}, 1.7 \mu \mathrm{mol})$ and peptide $7(1.8 \mathrm{mg}, 2.6 \mu \mathrm{mol})$ were dissolved in pyridine/acetic acid $(1 / 9, \mathrm{~mol} / \mathrm{mol})$ buffer at a concentration of $20 \mathrm{mM}$ at room temperature. The reaction mixture was stirred at room temperature for $7 \mathrm{~h}$. After completion of the reaction, the solvent was blown off under a stream of condensed air. The residue was then treated with $1.0 \mathrm{~mL}$ of $\mathrm{TFA} / \mathrm{H}_{2} \mathrm{O}(95 / 5, v / v)$ to afford crude peptide thioester 4c. TFA was blown off under a stream of condensed air. Without any purification, peptide $8(4.5 \mathrm{mg}, 5.2 \mu \mathrm{mol})$ in NCL buffer (prepared according to general experimental procedure E) was added into crude peptide thioester $\mathbf{4 c}$ (final concentration $=2 \mathrm{mM}$ ). The reaction mixture was stirred for $1 \mathrm{~h}$, and after completion of the ligation as assessed by HPLC, an aqueous solution of $\mathrm{MeONH}_{2} \cdot \mathrm{HCl}(29 \mu \mathrm{L}$, $0.6 \mathrm{M})$ was added followed by TCEP solution $(35 \mu \mathrm{L}, 0.5 \mathrm{M})$. The $\mathrm{pH}$ was adjusted to $\sim 4.0-4.5$ by addition of $2 \mathrm{M} \mathrm{HCl}$ solution and the turbid mixture was stirred for $18 \mathrm{~h}$. Preparative HPLC purification $\left(10-50 \% \mathrm{CH}_{3} \mathrm{CN} / \mathrm{H}_{2} \mathrm{O}\right.$ over $\left.30 \mathrm{~min}\right)$ followed by concentration at reduced pressure and lyophilization afforded $1.4 \mathrm{mg}$ (27\% yield) of peptide 9 as a white powder. 

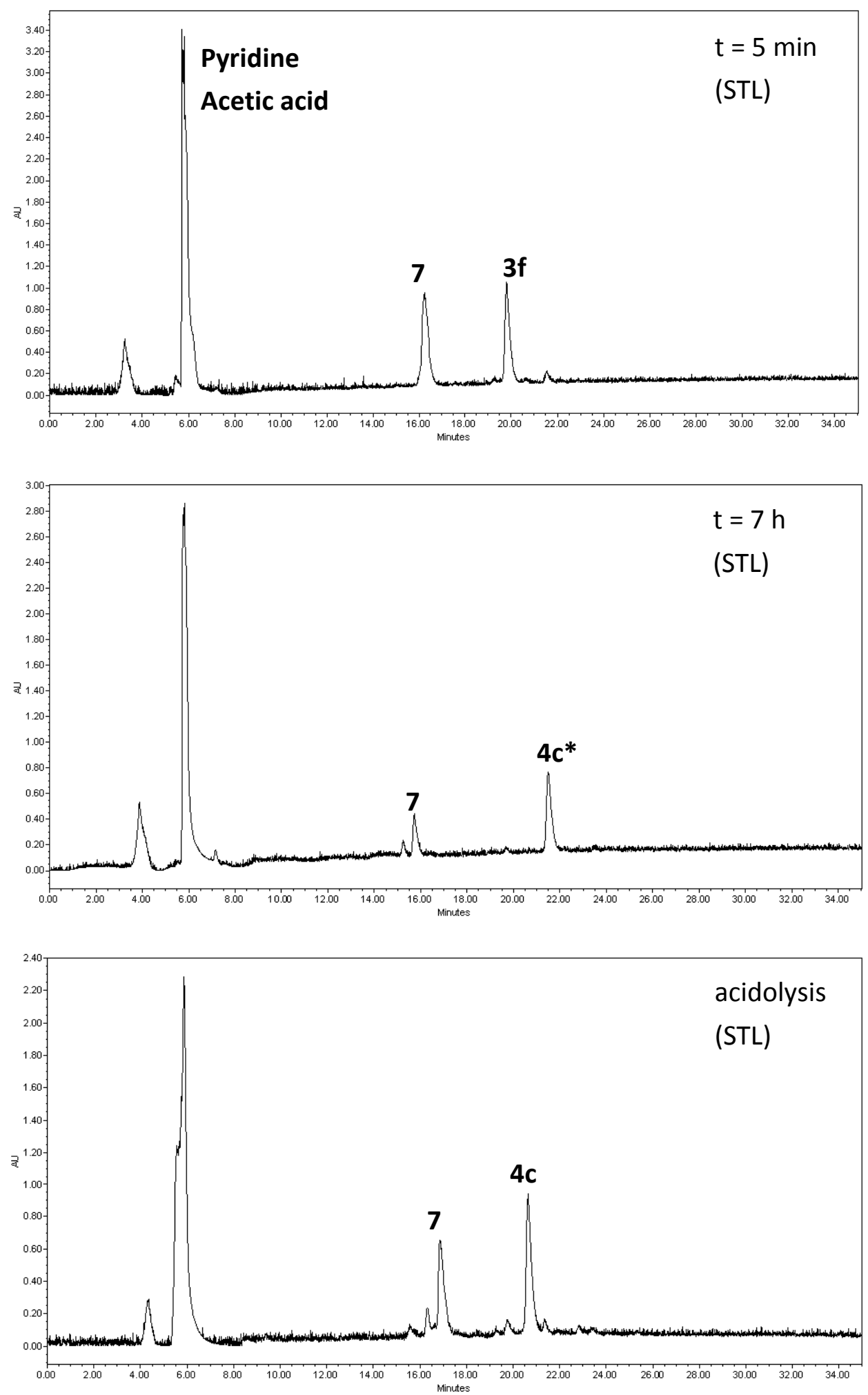


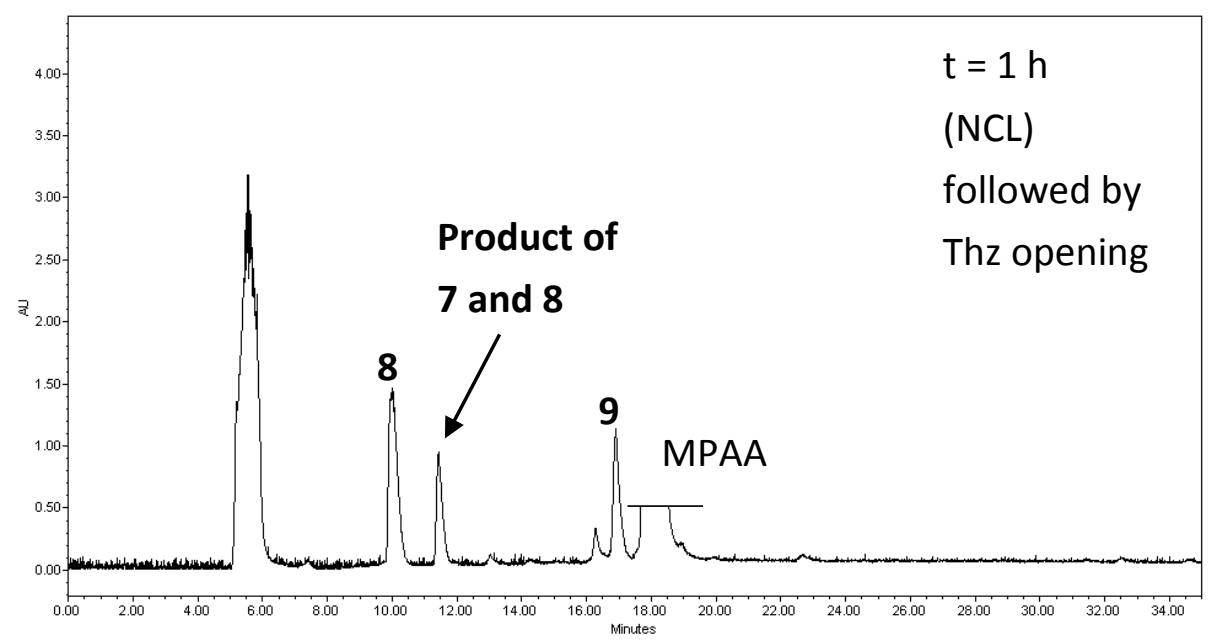

Figure S61. UV trace (190-400 nm) from LC-MS analysis of STL between $3 \mathbf{f}$ and 7 at $5 \mathrm{~min}, 7 \mathrm{~h}$ and acidolysis to give $4 \mathbf{c}$ and NCL between crude $4 \mathbf{c}$ and $\mathbf{8}$ at $1 \mathrm{~h}$ followed by Thz opening to generate 9: gradient 10-50\% $\mathrm{CH}_{3} \mathrm{CN} / \mathrm{H}_{2} \mathrm{O}$ containing $0.1 \%$ TFA over $30 \mathrm{~min}$ at a flow rate of $0.6 \mathrm{~mL} / \mathrm{min}$.

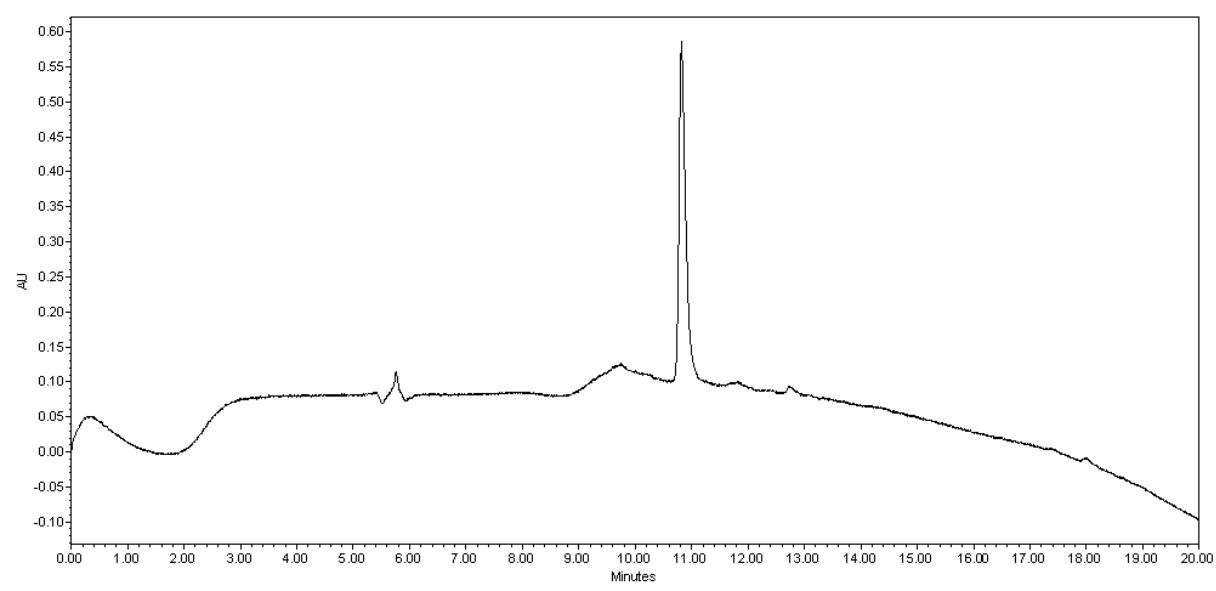

Figure S62. UV trace from analytical LC-MS analysis of purified peptide 9: gradient $5-95 \% \mathrm{CH}_{3} \mathrm{CN} / \mathrm{H}_{2} \mathrm{O}$ containing $0.1 \%$ TFA over $15 \mathrm{~min}$ at a flow rate of $0.6 \mathrm{~mL} / \mathrm{min}$. 


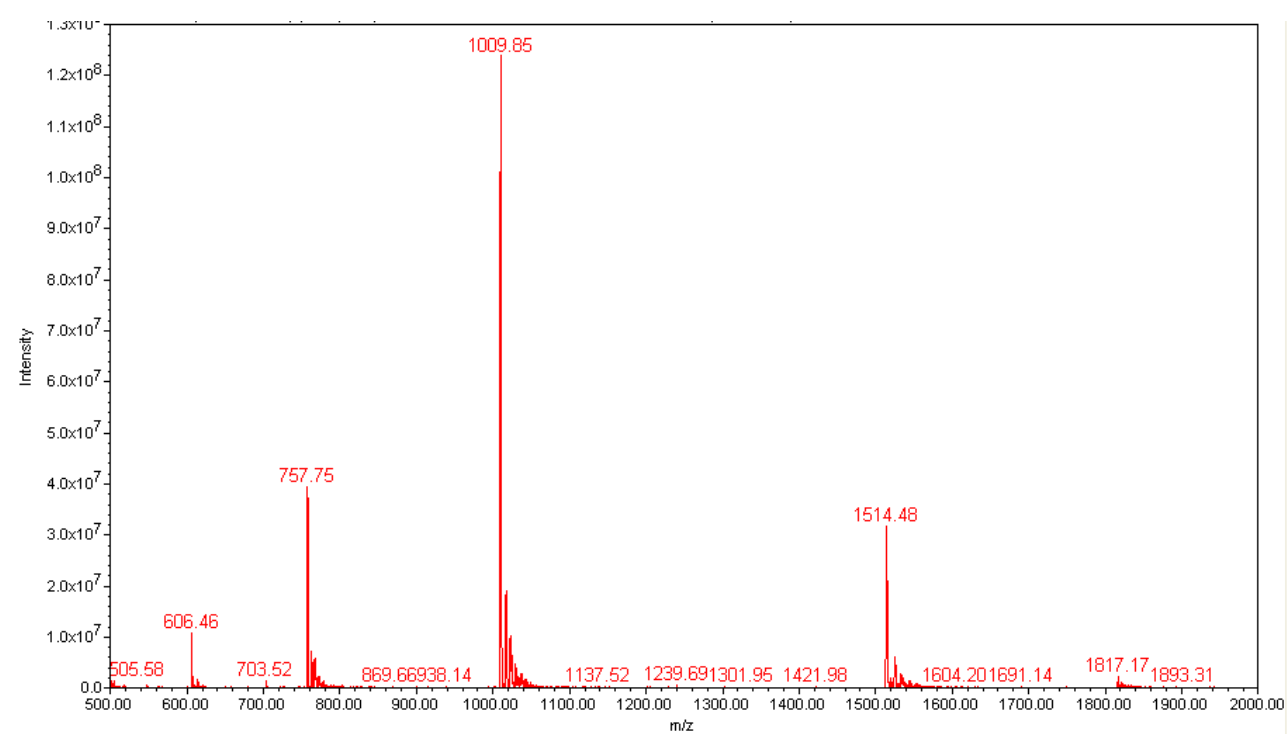

Figure S63. ESI-MS calcd. for $\mathrm{C}_{131} \mathrm{H}_{200} \mathrm{~N}_{38} \mathrm{O}_{41} \mathrm{~S}_{2}, 3027.35$, $[\mathrm{M}+2 \mathrm{H}]^{2+} m / z=1514.68$, found 1514.48; $[\mathrm{M}+3 \mathrm{H}]^{3+} \mathrm{m} / \mathrm{z}=1010.12$, found $1009.85 ;[\mathrm{M}+4 \mathrm{H}]^{4+} \mathrm{m} / \mathrm{z}=757.84$, found $757.75 ;[\mathrm{M}+5 \mathrm{H}]^{5+} \mathrm{m} / \mathrm{z}=606.48$, found 606.46 . 


\section{$\underline{X}$. Total synthesis of glycosylated IL-25 by $N$-to- $C$ STL strategy and One-pot Three-segment STL and NCL}

\section{A. Preparation of IL-25 glycopeptide/peptide fragments}

Preparation of peptide hydrazide 10: IL-25 (1-41) ${ }^{2}$

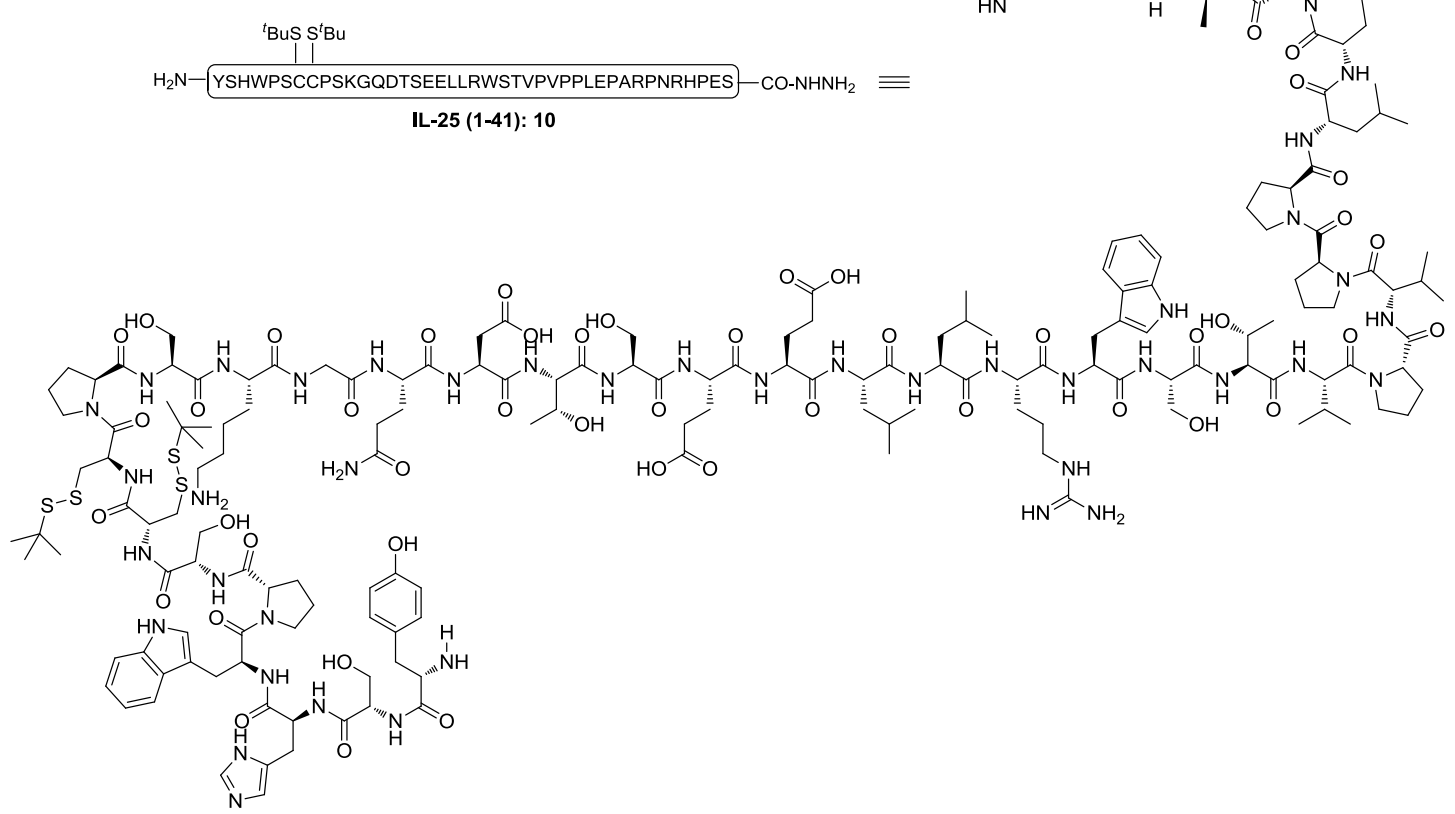

IL-25 (1-41) (10) was synthesized according to the general procedure A using hydrazine 2-chlorotrityl chloride resin (0.5 g) (prepared according to general experimental procedure F). Upon completion of synthesis, the peptide resin was subjected to a cleavage cocktail of TFA/ $\mathrm{H}_{2} \mathrm{O} / \mathrm{TIPS}(95 / 2.5 / 2.5, v / v / v)$ for $2 \mathrm{~h}$. The resin was filtered and the combined filtrates were blown off under a stream of condensed air. The crude product was triturated with cold diethyl ether to give a white 
suspension for centrifuge. After decanting diethyl ether, the remaining solid was subjected to preparative HPLC purification $\left(30-40 \% \mathrm{CH}_{3} \mathrm{CN} / \mathrm{H}_{2} \mathrm{O}\right.$ over $\left.30 \mathrm{~min}\right)$ and lyophilization to give $\mathbf{1 0}(85.6 \mathrm{mg}, 7 \%)$ as a white powder.

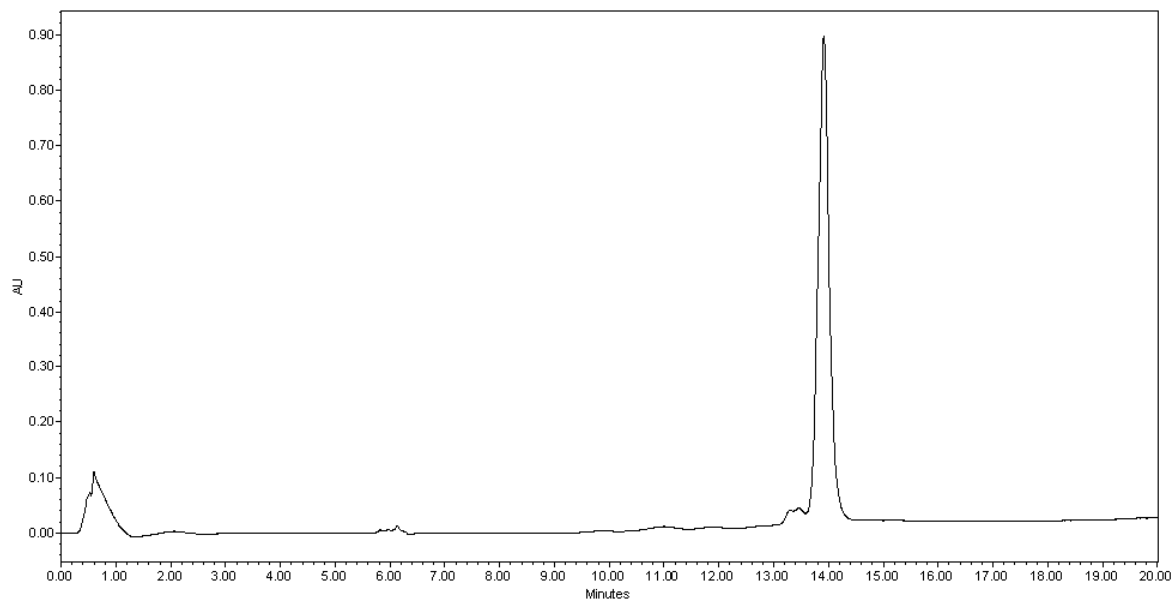

Figure S64. UV trace from LC-MS analysis of purified 10: gradient 5-95\% $\mathrm{CH}_{3} \mathrm{CN} / \mathrm{H}_{2} \mathrm{O}$ containing $0.1 \%$ TFA over $15 \mathrm{~min}$ at a flow rate of $0.6 \mathrm{~mL} / \mathrm{min}$.

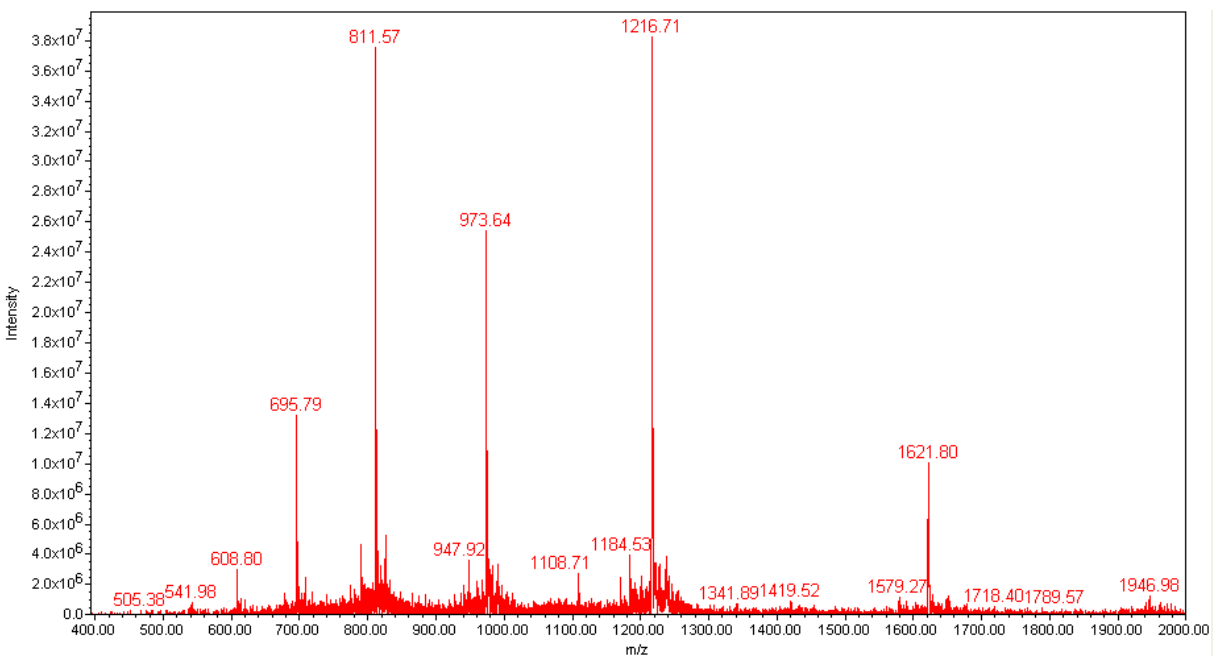

Figure S65. ESI-MS calcd. for $\mathrm{C}_{213} \mathrm{H}_{327} \mathrm{~N}_{61} \mathrm{O}_{62} \mathrm{~S}_{4}, 4862.51$ Da (average isotopes), $[\mathrm{M}+3 \mathrm{H}]^{3+} \mathrm{m} / \mathrm{z}=1621.67$, found 1621.80; $[\mathrm{M}+4 \mathrm{H}]^{4+} \mathrm{m} / \mathrm{z}=1216.63$, found 1216.71; $[\mathrm{M}+5 \mathrm{H}]^{5+} \mathrm{m} / \mathrm{z}=973.50$, found 973.64; $[\mathrm{M}+6 \mathrm{H}]^{6+} \mathrm{m} / \mathrm{z}=811.42$, found 811.57; $[\mathrm{M}+7 \mathrm{H}]^{7+} \mathrm{m} / \mathrm{z}=695.64$, found 695.79; $[\mathrm{M}+8 \mathrm{H}]^{8+} \mathrm{m} / \mathrm{z}=608.81$, found 608.80 . 
Preparation of peptide hydrazide 11: IL-25 (42-79) ${ }^{2}$

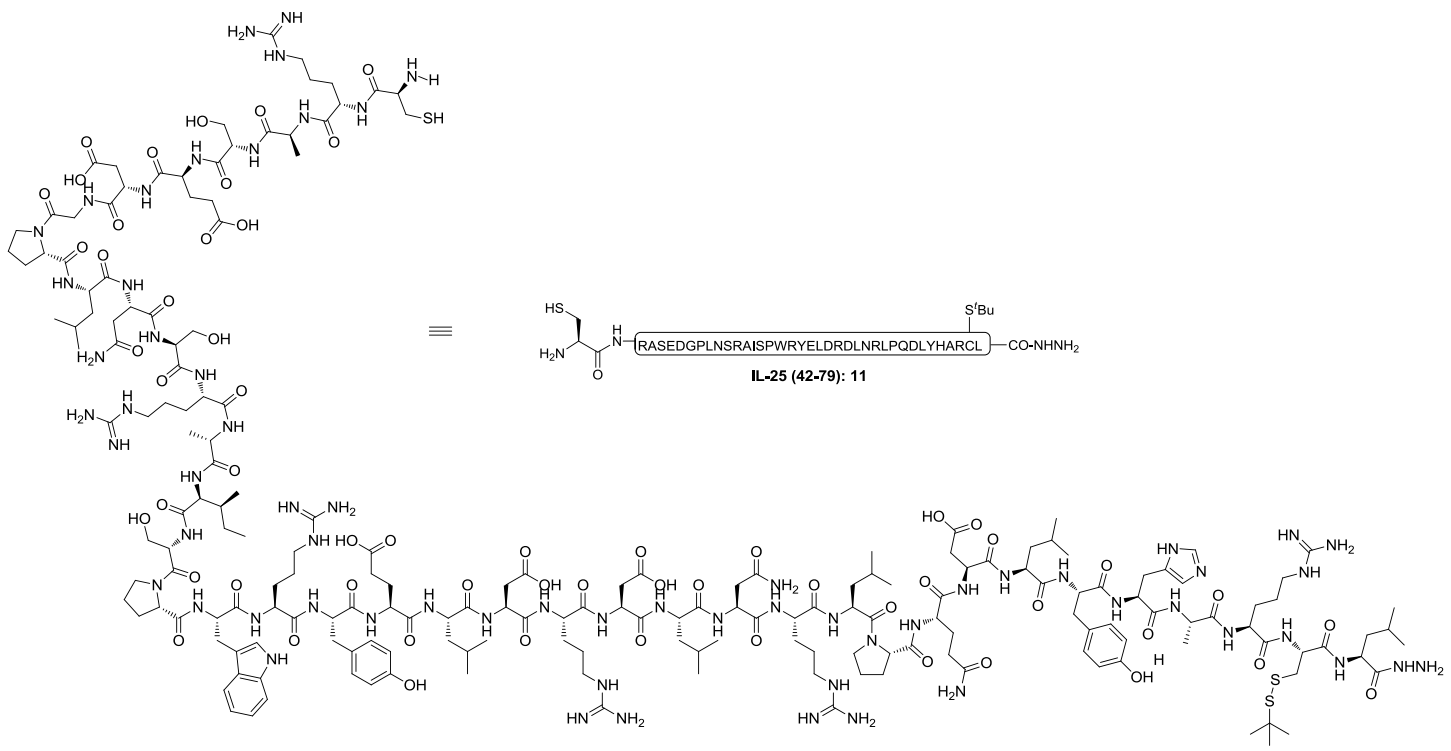

Peptide 11 was synthesized according to the general procedure A using hydrazine 2-chlorotrityl chloride resin $(0.5 \mathrm{~g})$ (prepared according to general experimental procedure F). Upon completion of synthesis, the peptide resin was subjected to a cleavage cocktail of TFA/ $\mathrm{H}_{2} \mathrm{O} / \mathrm{TIPS}(95 / 2.5 / 2.5, v / v / v)$ for $2 \mathrm{~h}$. The resin was filtered and the combined filtrates were blown off under a stream of condensed air. The crude product was triturated with cold diethyl ether to give a white suspension for centrifuge. After decanting diethyl ether, the remaining solid was subjected to preparative HPLC purification $\left(25-40 \% \mathrm{CH}_{3} \mathrm{CN} / \mathrm{H}_{2} \mathrm{O}\right.$ over $\left.30 \mathrm{~min}\right)$ and lyophilization to give 11 (102.6 $\mathrm{mg}, 9 \%)$ as a white powder.

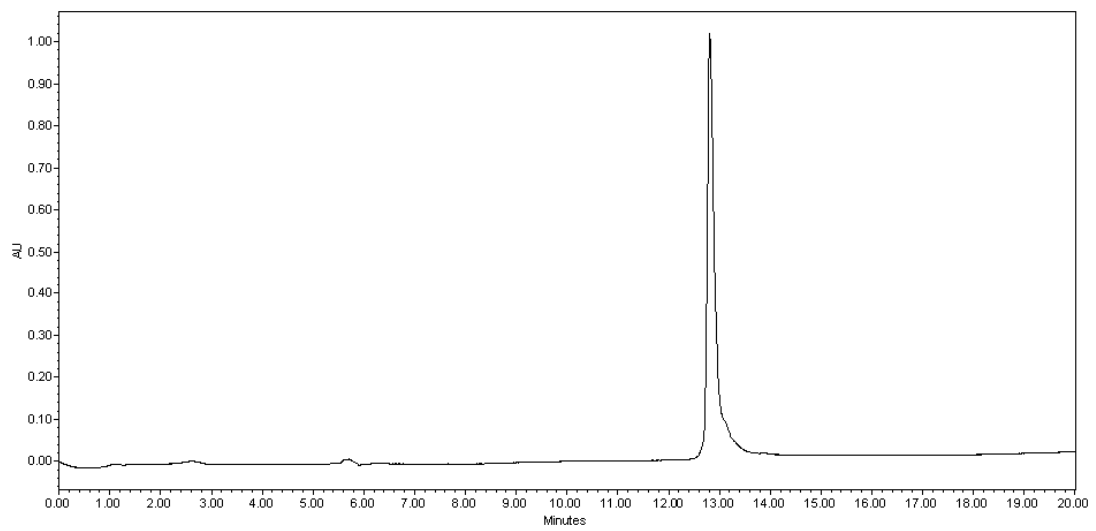

Figure S66. UV trace from LC-MS analysis of purified 11: gradient 5-95\% $\mathrm{CH}_{3} \mathrm{CN} / \mathrm{H}_{2} \mathrm{O}$ containing $0.1 \%$ TFA over $15 \mathrm{~min}$ at a flow rate of $0.6 \mathrm{~mL} / \mathrm{min}$. 


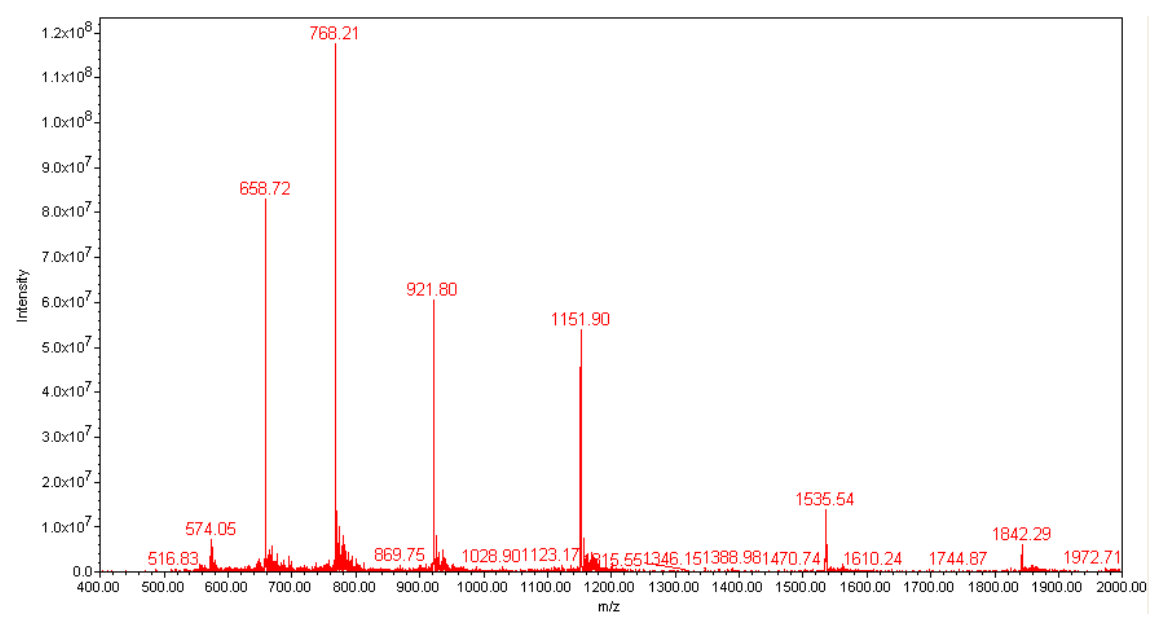

Figure S67. ESI-MS calcd. for $\mathrm{C}_{197} \mathrm{H}_{314} \mathrm{~N}_{64} \mathrm{O}_{58} \mathrm{~S}_{3}, 4603.19$ Da (average isotopes), $[2 \mathrm{M}+5 \mathrm{H}]^{5+} \mathrm{m} / \mathrm{z}=1842.28$, found 1842.29; $[\mathrm{M}+3 \mathrm{H}]^{3+} \mathrm{m} / \mathrm{z}=1535.40$, found 1535.54; $[\mathrm{M}+4 \mathrm{H}]^{4+} \mathrm{m} / \mathrm{z}=1151.80$, found 1151.90; $[\mathrm{M}+5 \mathrm{H}]^{5+} \mathrm{m} / \mathrm{z}=921.64$, found 921.80; $[\mathrm{M}+6 \mathrm{H}]^{6+} \mathrm{m} / \mathrm{z}=768.21$, found $768.2 ;[\mathrm{M}+7 \mathrm{H}]^{7+} \mathrm{m} / \mathrm{z}=658.60$, found 658.72 .

\section{Preparation of C-terminal peptide SAL ${ }^{\text {on }}$ ester 12: IL-25 (80-89)}

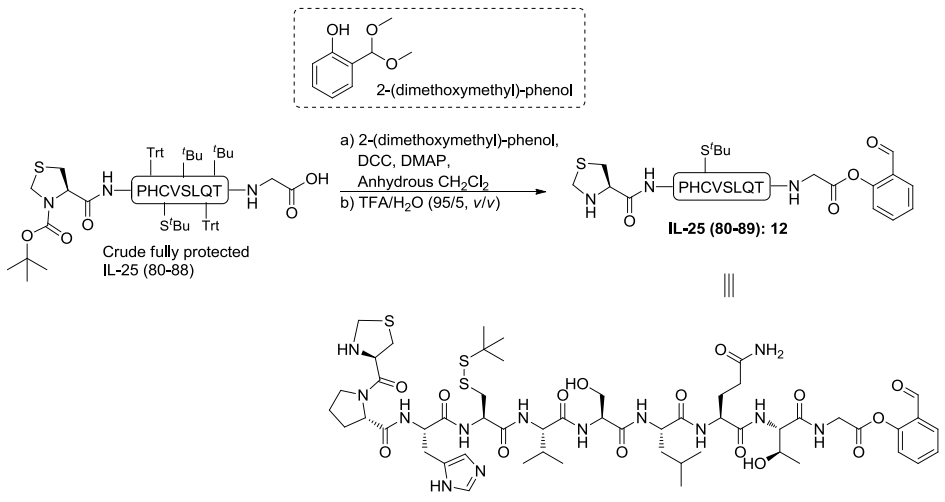

Scheme S11. Synthesis of (12).

Crude fully protected IL-25 (80-88) bearing C-terminus free carboxylic acid was firstly synthesized according to the general procedure A and B using 2-chlorotrityl chloride resin. The fully protected peptidyl acid $(100.0 \mathrm{mg}, 54.4 \mu \mathrm{mol})$ was coupled with 2-(dimethoxymethyl)-phenol (182.7 $\mathrm{mg}, 1087.5 \quad \mu \mathrm{mol})$, $N, N^{\prime}$-dicyclohexylcarbodiimide $\quad(\mathrm{DCC}) \quad(56.1 \quad \mathrm{mg}, \quad 271.9 \quad \mu \mathrm{mol}) \quad$ and 4-dimethylaminopyridine (DMAP) $(33.2 \mathrm{mg}, 271.9 \mu \mathrm{mol})$ in anhydrous $\mathrm{CH}_{2} \mathrm{Cl}_{2}$ overnight. Upon completion, the reaction mixture was concentrated and subjected to the treatment with TFA/ $\mathrm{H}_{2} \mathrm{O}(95 / 5, v / v)$ for $2 \mathrm{~h}$. The crude peptide salicylaldehyde 
ester was precipitated out by diethyl ether. Preparative HPLC purification (25-45\% $\mathrm{CH}_{3} \mathrm{CN} / \mathrm{H}_{2} \mathrm{O}$ over $30 \mathrm{~min}$ ) followed by concentration at reduced pressure and lyophilization afforded peptide $12(67.9 \mathrm{mg}, 31 \%$ yield) as a white powder.

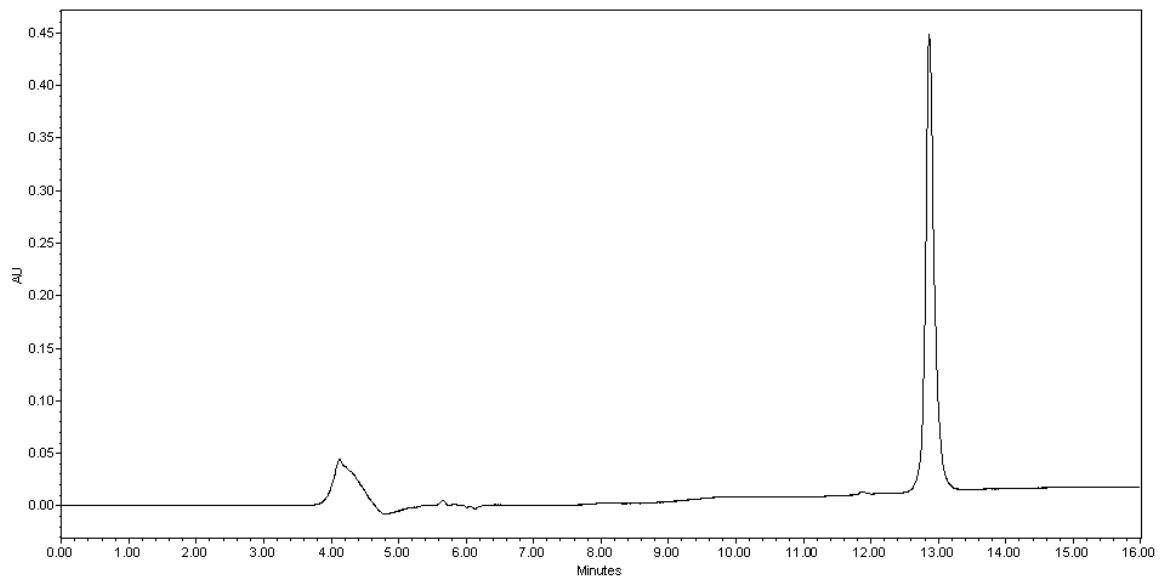

Figure S68. UV trace $(190-400 \mathrm{~nm})$ from LC-MS analysis of purified 12: gradient $5-95 \% \mathrm{CH}_{3} \mathrm{CN} / \mathrm{H}_{2} \mathrm{O}$ containing $0.1 \%$ TFA over $15 \mathrm{~min}$ at a flow rate of $0.6 \mathrm{~mL} / \mathrm{min}$.

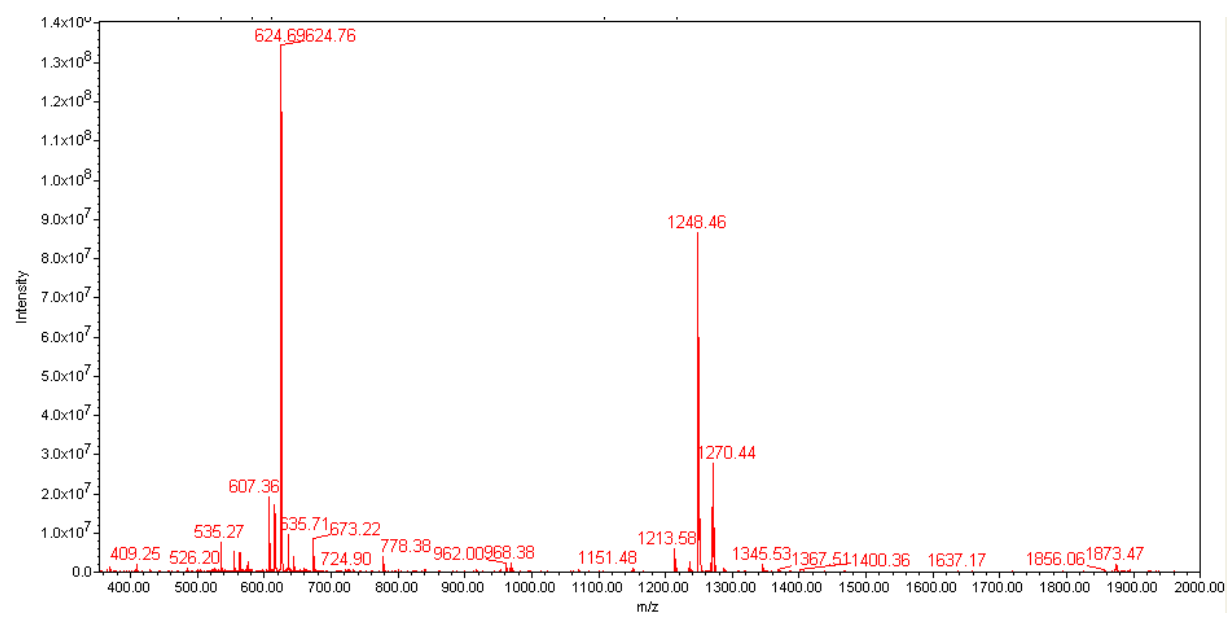

Figure S69. ESI-MS calcd. for $\mathrm{C}_{54} \mathrm{H}_{82} \mathrm{~N}_{13} \mathrm{O}_{15} \mathrm{~S}_{3},[\mathrm{M}+\mathrm{H}]^{+} m / z=1248.52$, found 1248.46; $[\mathrm{M}+\mathrm{Na}]^{+} \mathrm{m} / \mathrm{z}=1270.50$, found 1270.44; $[\mathrm{M}+2 \mathrm{H}]^{2+} \mathrm{m} / \mathrm{z}=624.76$, found 624.76 . 


\section{Synthesis of C-terminal glycopeptide SAL ${ }^{\text {off }}$ esters 13: IL-25 (90-118)}

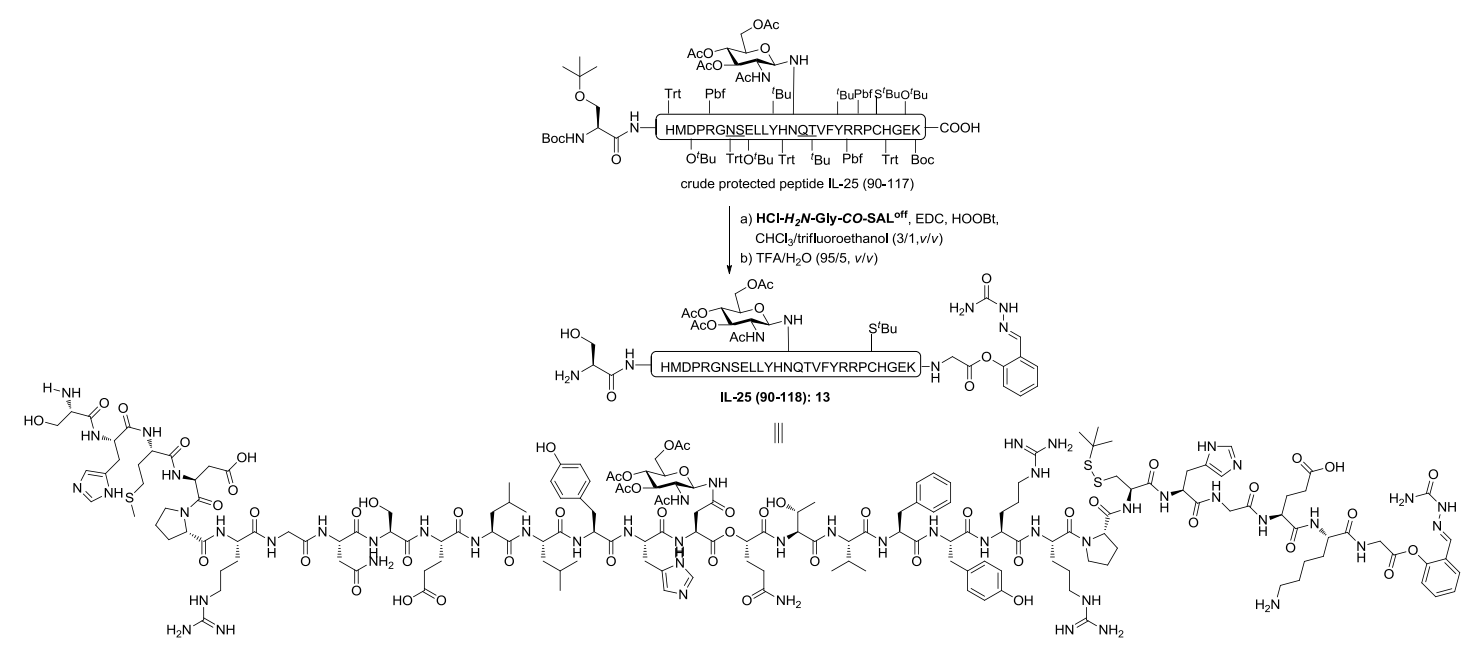

Scheme S12. Synthesis of (13) by $n+1$ strategy. Pseudoproline dipeptides are involved during SPPS (underlined).

Crude fully protected IL-25 (90-117) bearing C-terminus free carboxylic acid was firstly synthesized according to the general procedure A and B using 2-chlorotrityl chloride resin. The fully protected peptidyl acid $(150.5 \mathrm{mg}, 23.5 \mu \mathrm{mol})$ was dissolved in $\mathrm{CHCl}_{3} /$ trifluoroethanol $(1.6 \mathrm{~mL})$, and EDC (10.9 mg, $\left.70.5 \mu \mathrm{mol}\right)$ and HOOBt (11.5 $\mathrm{mg}, 70.5 \mu \mathrm{mol})$ were then added followed by $\mathbf{H C l} \cdot \boldsymbol{H}_{2} \boldsymbol{N}$-Gly-CO-SAL ${ }^{\text {off }}(19.2$ $\mathrm{mg}, 70.5 \mu \mathrm{mol})$, as described in general procedure $\mathrm{D}$. The reaction mixture stirred for $3 \mathrm{~h}$ to form the crude protected C-terminal peptide SAL ${ }^{\text {off }}$ ester. Upon completion, the reaction mixture was concentrated and subjected to treatment with TFA/ $\mathrm{H}_{2} \mathrm{O}(95 / 5$, $v / v$ ). After stirring for $2 \mathrm{~h}$, the solution was evaporated under a stream of nitrogen to half of the initial volume. The residue was treated with ice-cold diethyl ether ( $45 \mathrm{~mL})$, and the resulting suspension was centrifuged to give a white pellet. The supernant was decanted and the pellet was triturated with ice-cold diethyl ether $(45 \mathrm{~mL})$. This process was repeated three times in total. Preparative HPLC purification (23-30\% $\mathrm{CH}_{3} \mathrm{CN} / \mathrm{H}_{2} \mathrm{O}$ over $30 \mathrm{~min}$ ) followed by concentration at reduced pressure and lyophilization afforded peptide $\mathbf{1 3}$ (26.4 $\mathrm{mg}, 28 \%$ yield) as a white powder. 


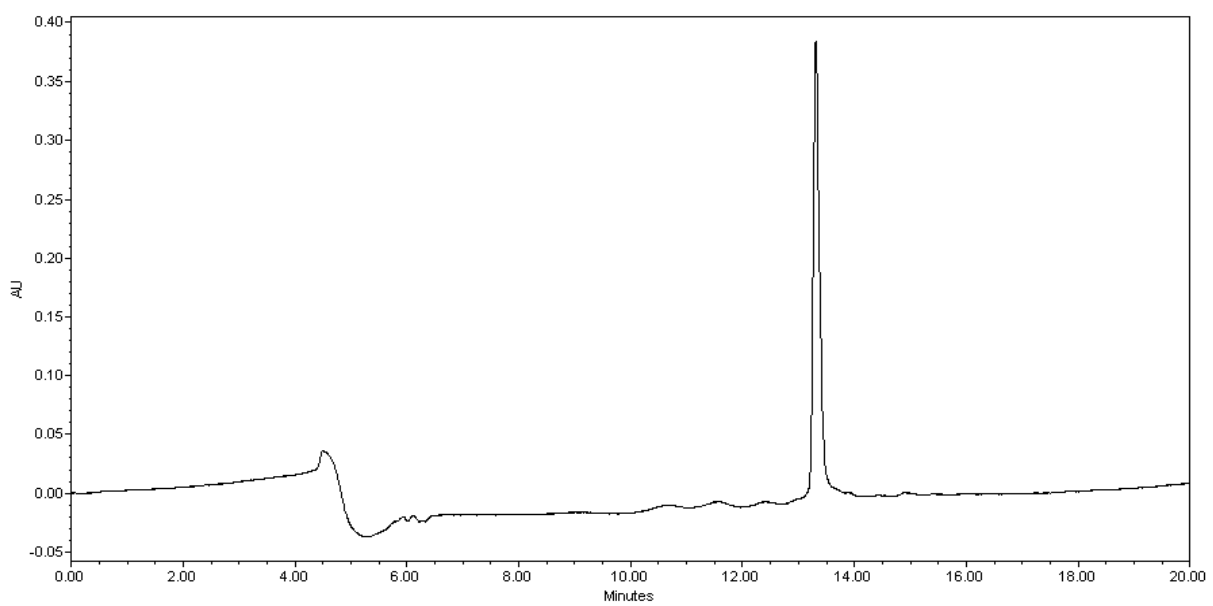

Figure S70. UV trace $(190-400 \mathrm{~nm})$ from LC-MS analysis of purified 13: gradient $5-95 \% \mathrm{CH}_{3} \mathrm{CN} / \mathrm{H}_{2} \mathrm{O}$ containing $0.1 \%$ TFA over $15 \mathrm{~min}$ at a flow rate of $0.6 \mathrm{~mL} / \mathrm{min}$.

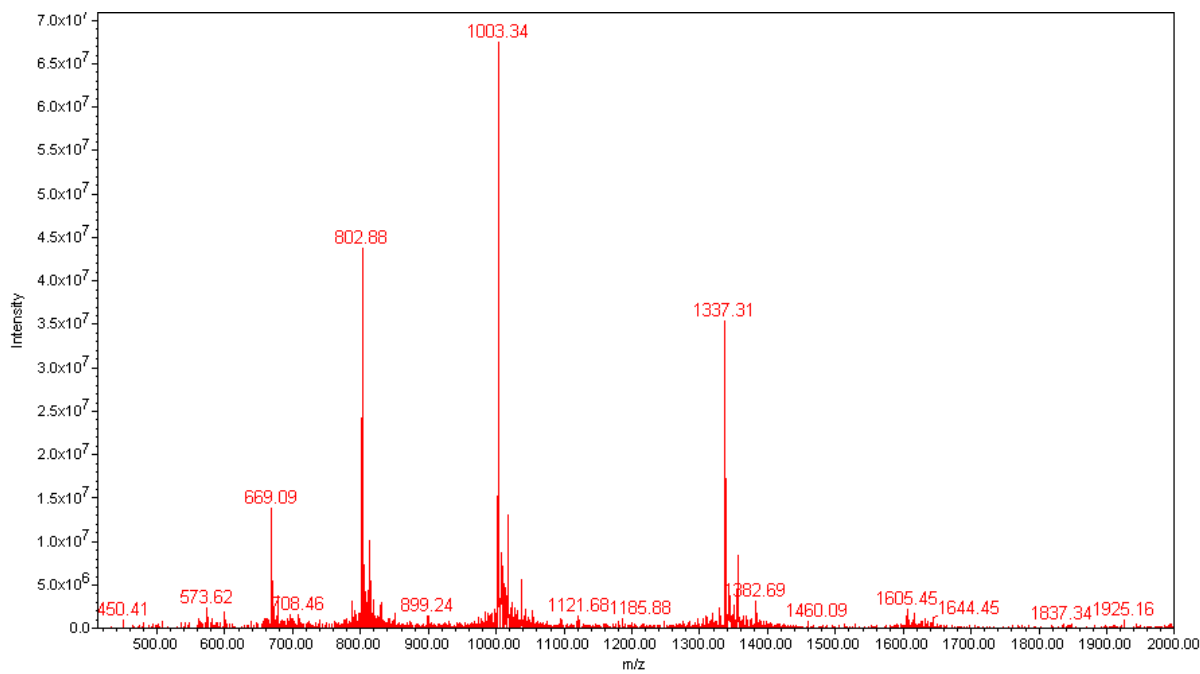

Figure S71. ESI-MS calcd. for $\mathrm{C}_{173} \mathrm{H}_{256} \mathrm{~N}_{52} \mathrm{O}_{53} \mathrm{~S}_{3}, 4008.40 \mathrm{Da}$ (average isotopes) $[\mathrm{M}+3 \mathrm{H}]^{3+} \mathrm{m} / \mathrm{z}=1337.13$, found 1337.31; $[\mathrm{M}+4 \mathrm{H}]^{4+} \mathrm{m} / \mathrm{z}=1003.10$, found 1003.34; $[\mathrm{M}+5 \mathrm{H}]^{5+} \mathrm{m} / \mathrm{z}=802.68$, found 802.88; $[\mathrm{M}+6 \mathrm{H}]^{6+} \mathrm{m} / \mathrm{z}=669.07$, found 669.09. 
Synthesis of C-terminal peptide thioesters 15: IL-25 (119-123) ${ }^{1}$

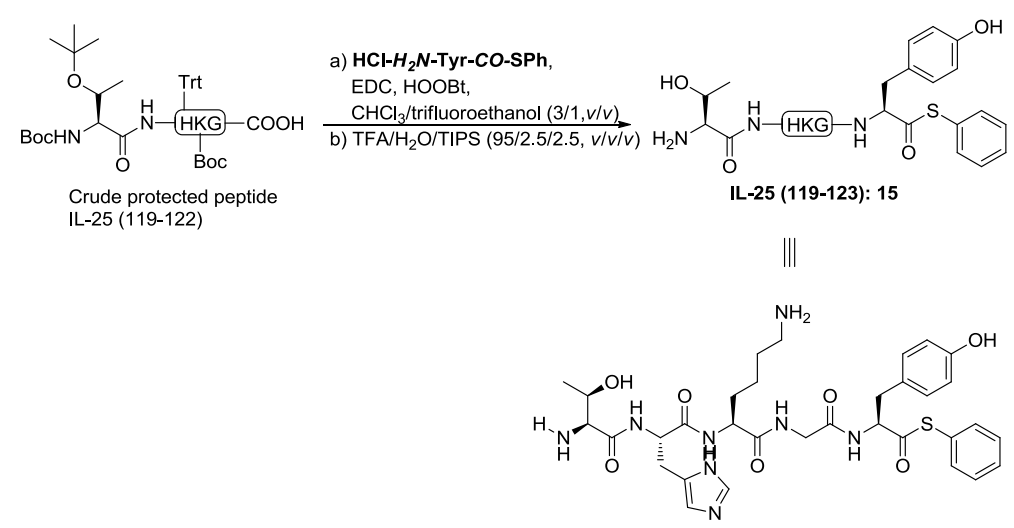

Scheme S13. Synthesis of (15) by $n+1$ strategy.

Crude protected peptide $\mathrm{BocHN}-\mathrm{Ser}\left({ }^{t} \mathrm{Bu}\right)-\mathrm{His}(\mathrm{Trt})-\mathrm{Lys}(\mathrm{Boc})-\mathrm{Gly}-\mathrm{COOH}$ (prepared according to general experimental procedure A and B) $(55.3 \mathrm{mg}, 73.4 \mu \mathrm{mol})$ was dissolved in $\mathrm{CHCl}_{3}$ /trifluoroethanol $(7.4 \mathrm{~mL})$, and $\mathrm{EDC}(34.1 \mathrm{mg}, 220.1 \mu \mathrm{mol})$ and HOOBt $(35.9 \mathrm{mg}, 220.1 \mu \mathrm{mol})$ were then added. After $5 \mathrm{~min}$, HCl$\cdot H_{2} \boldsymbol{N}$-Tyr-SPh (70.0 mg, $220.1 \mu \mathrm{mol}$ ) was added, and the reaction mixture was stirred for $3 \mathrm{~h}$ to form the crude protected $\mathrm{C}$-terminal peptide thioester. The reaction mixture was then concentrated and subjected to $5.0 \mathrm{~mL}$ of TFA/ $\mathrm{H}_{2} \mathrm{O} / \mathrm{TIPS}(95 / 2.5 / 2.5, v / v / v)$. After stirring for $2 \mathrm{~h}$, the solution was evaporated under a stream of nitrogen to half of the initial volume. The residue was treated with ice-cold diethyl ether $(45 \mathrm{~mL})$, and the resulting suspension was centrifuged to give a white pellet. The supernant was decanted and the pellet was triturated with ice-cold diethyl ether $(45 \mathrm{~mL})$. Preparative HPLC purification (20-30\% $\mathrm{CH}_{3} \mathrm{CN} / \mathrm{H}_{2} \mathrm{O}$ over $30 \mathrm{~min}$ ) followed by concentration at reduced pressure and lyophilization to give $\mathbf{1 5}(17.4 \mathrm{mg}, 34 \%$ yield) as a white powder. 


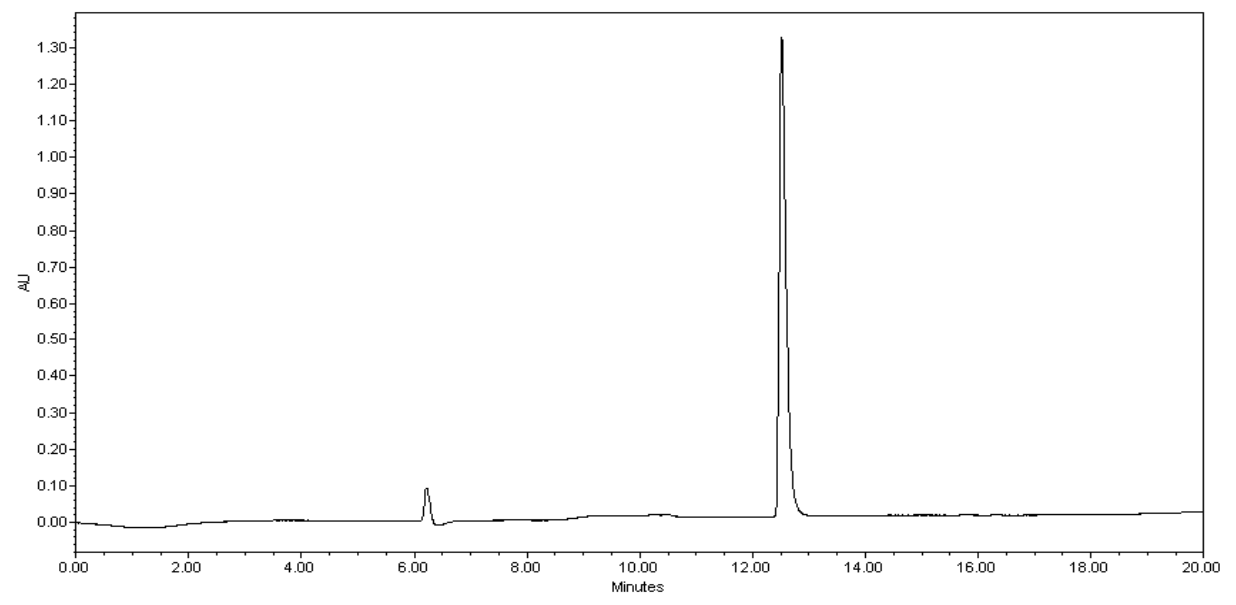

Figure S72. UV trace $(190-400 \mathrm{~nm})$ from LC-MS analysis of purified 15: gradient $5-95 \% \mathrm{CH}_{3} \mathrm{CN} / \mathrm{H}_{2} \mathrm{O}$ containing $0.1 \%$ TFA over $15 \mathrm{~min}$ at a flow rate of $0.6 \mathrm{~mL} / \mathrm{min}$.

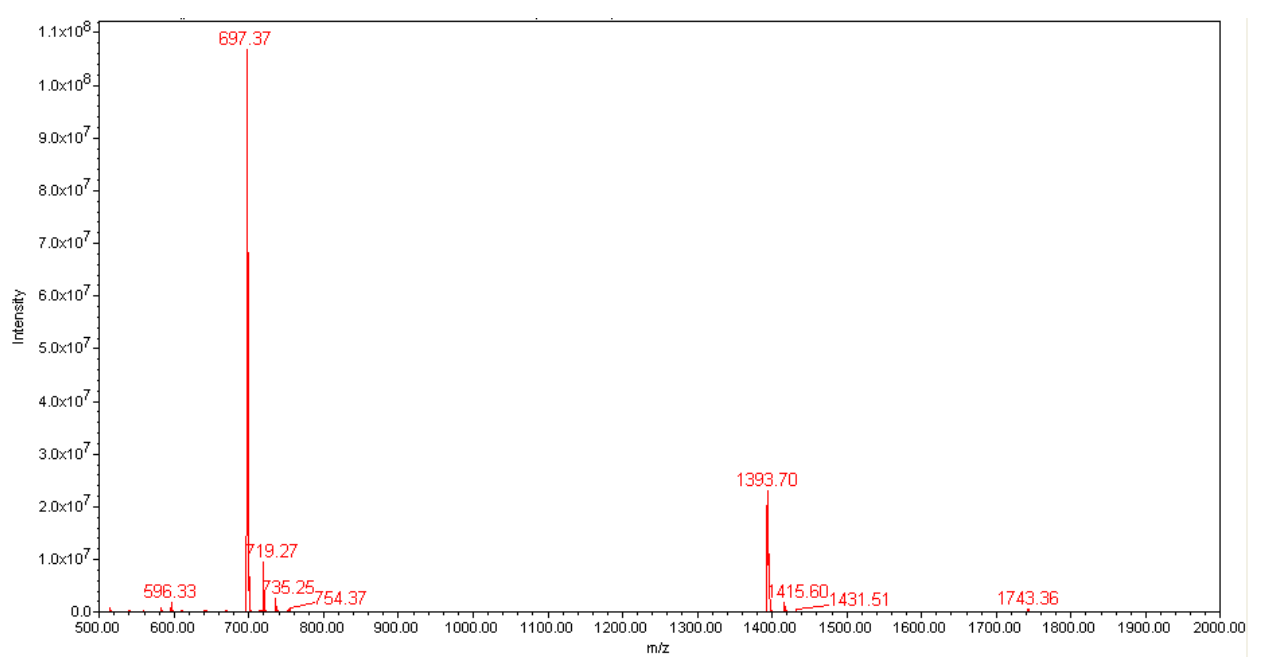

Figure S73. ESI-MS calcd. for $\mathrm{C}_{33} \mathrm{H}_{45} \mathrm{~N}_{8} \mathrm{O}_{7} \mathrm{~S}[\mathrm{M}+\mathrm{H}]^{+} m / z=697.31$, found 697.37; $[\mathrm{M}+\mathrm{Na}]^{+} \mathrm{m} / z=719.29$, found $719.27[2 \mathrm{M}+\mathrm{H}]^{+} \mathrm{m} / z=1393.62$, found 1393.70. 


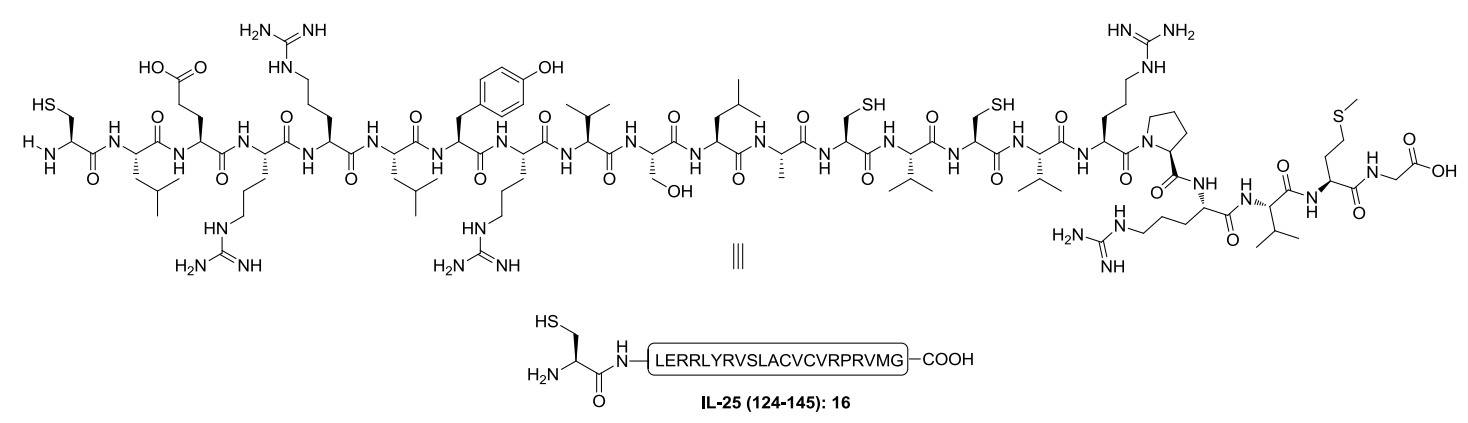

Peptide 16 was synthesized according to the general procedure A using 2-chlorotrityl chloride resin $(0.5 \mathrm{~g})$. Upon completion of synthesis, the peptide resin was subjected to a cleavage of TFA/ $\mathrm{H}_{2} \mathrm{O} / \mathrm{TIPS}(95 / 2.5 / 2.5, v / v / v)$ for $2 \mathrm{~h}$. The resin was filtered and the combined filtrates were blown off under a stream of condensed air. The crude product was triturated with cold diethyl ether to give a white suspension for centrifuge. After decanting diethyl ether, the remaining solid was subjected to preparative HPLC purification (20-35\% $\mathrm{CH}_{3} \mathrm{CN} / \mathrm{H}_{2} \mathrm{O}$ over $30 \mathrm{~min}$ ) followed by concentration at reduced pressure and lyophilization to afford $\mathbf{1 6}(22.5 \mathrm{mg}, 4 \%)$ as a white powder.

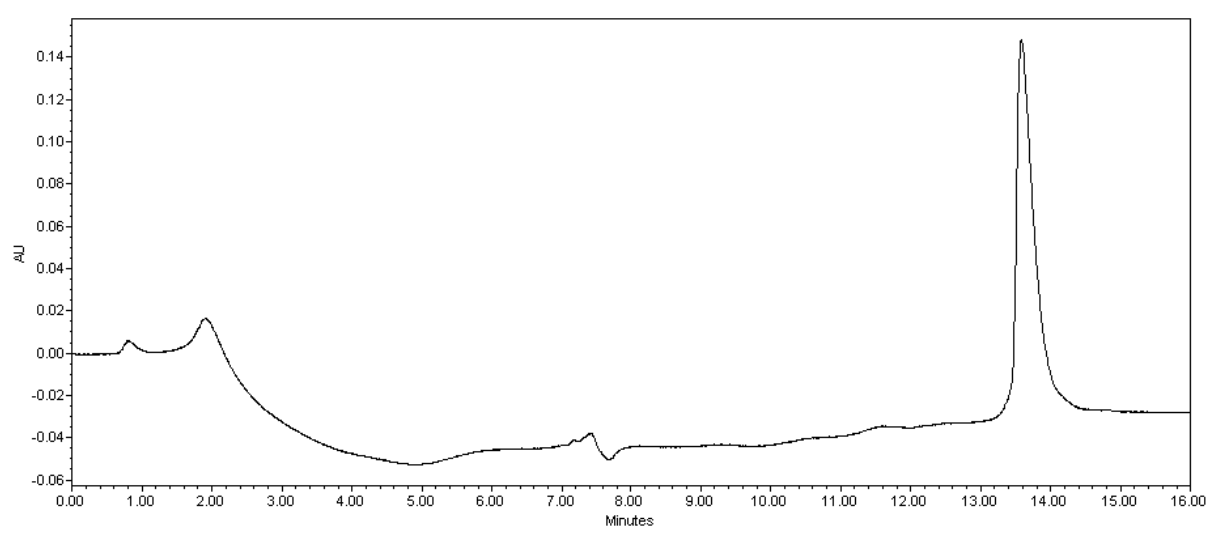

Figure S74. UV trace $(190-400 \mathrm{~nm})$ from LC-MS analysis of purified 16: gradient $5-95 \% \mathrm{CH}_{3} \mathrm{CN} / \mathrm{H}_{2} \mathrm{O}$ containing $0.1 \%$ TFA over $15 \mathrm{~min}$ at a flow rate of $0.6 \mathrm{~mL} / \mathrm{min}$. 


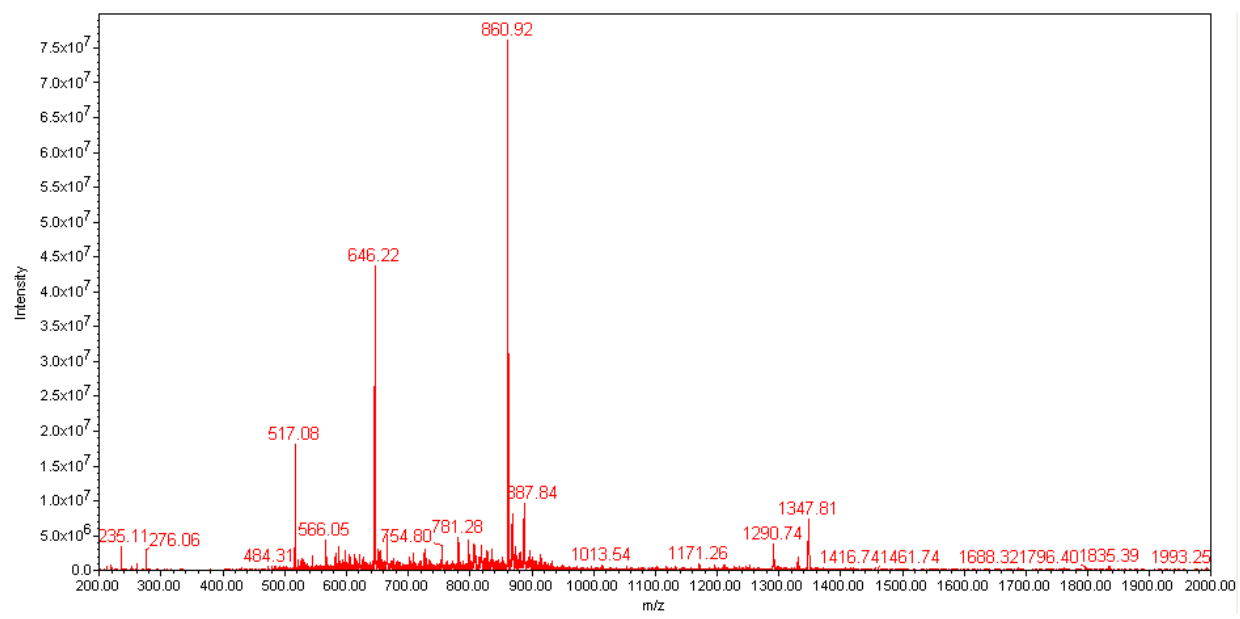

Figure S75. ESI-MS calcd. for $\mathrm{C}_{109} \mathrm{H}_{191} \mathrm{~N}_{37} \mathrm{O}_{27} \mathrm{~S}_{4}, 2580.17$ Da (average isotopes), $[\mathrm{M}+2 \mathrm{H}]^{2+} \mathrm{m} / \mathrm{z}=1291.09$, found $1290.74 ;[\mathrm{M}+3 \mathrm{H}]^{3+} \mathrm{m} / \mathrm{z}=861.07$, found 860.92; $[\mathrm{M}+4 \mathrm{H}]^{4+} \mathrm{m} / \mathrm{z}=646.05$, found $646.22 ;[\mathrm{M}+5 \mathrm{H}]^{5+} \mathrm{m} / z=517.04$, found 517.08.

\section{B. Assembly of IL-25 glycopeptide/peptide fragments}

Peptide fragment 14: IL-25 (80-118)

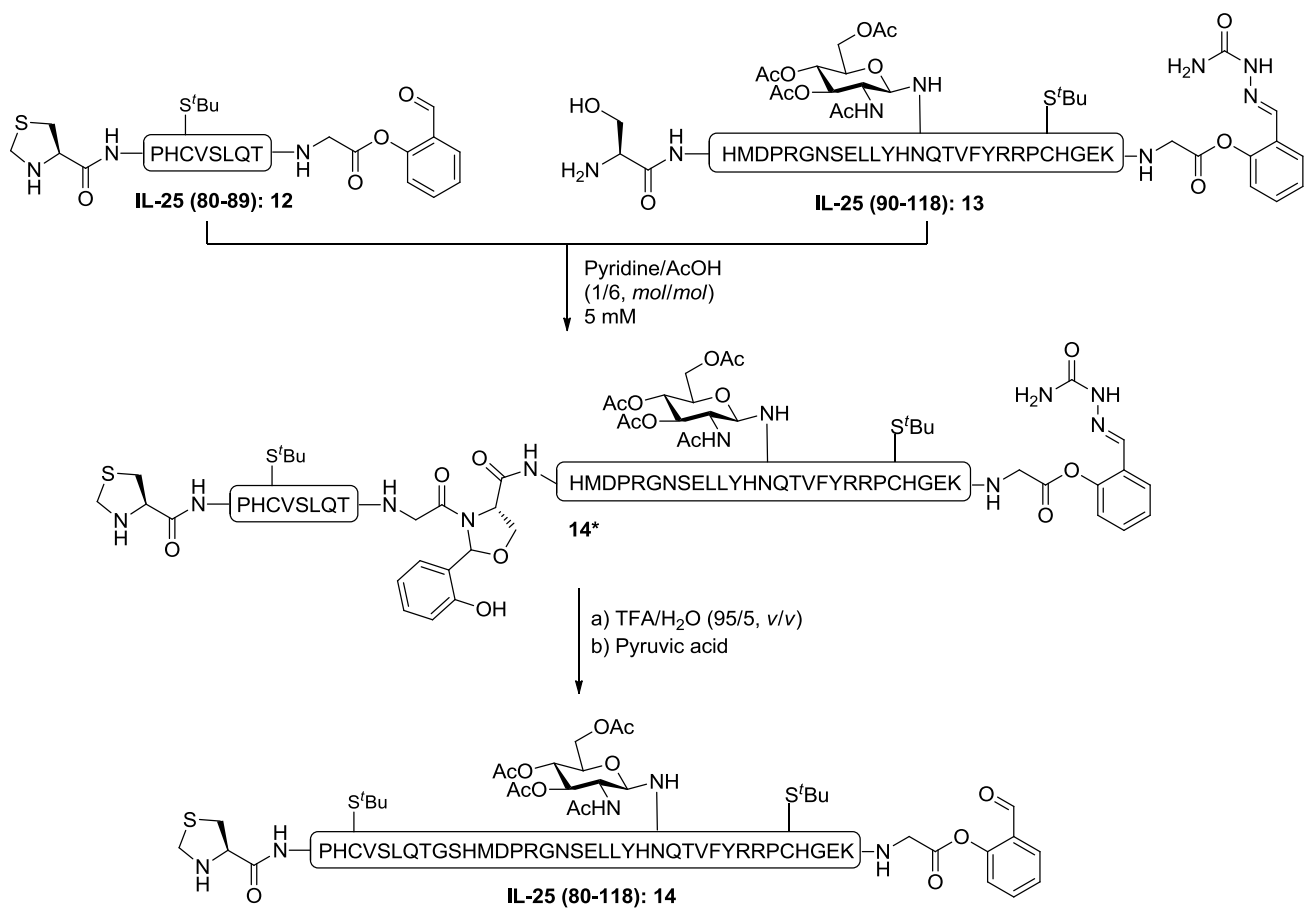

Scheme S14. Serine Ligation between (12) and (13) to generate (14) in $N$-to-C direction. 
12 (12.3 mg, $9.9 \mu \mathrm{mol})$ and $\mathbf{1 3}(26.4 \mathrm{mg}, 6.6 \mu \mathrm{mol})$ were dissolved in pyridine/acetic acid $(1 / 6$, mole/mole) buffer at a concentration of $5 \mathrm{mM}$ at room temperature. The reaction mixture was stirred at room temperature for $6 \mathrm{~h}$, and the solvent was then blown off under a stream of condensed air. The residue was then treated with $1.0 \mathrm{~mL}$ of TFA $/ \mathrm{H}_{2} \mathrm{O}(95 / 5, v / v)$ for $5 \mathrm{~min}$ followed by pyruvic acid $(4.6 \mu \mathrm{L}, 65.9 \mu \mathrm{mol})$ for 1 h. Preparative HPLC purification $\left(25-30 \% \mathrm{CH}_{3} \mathrm{CN} / \mathrm{H}_{2} \mathrm{O}\right.$ over $30 \mathrm{~min}$ ) followed by concentration at reduced pressure and lyophilization afforded $13.4 \mathrm{mg}$ (40\% yield) of 14 as a white solid. 

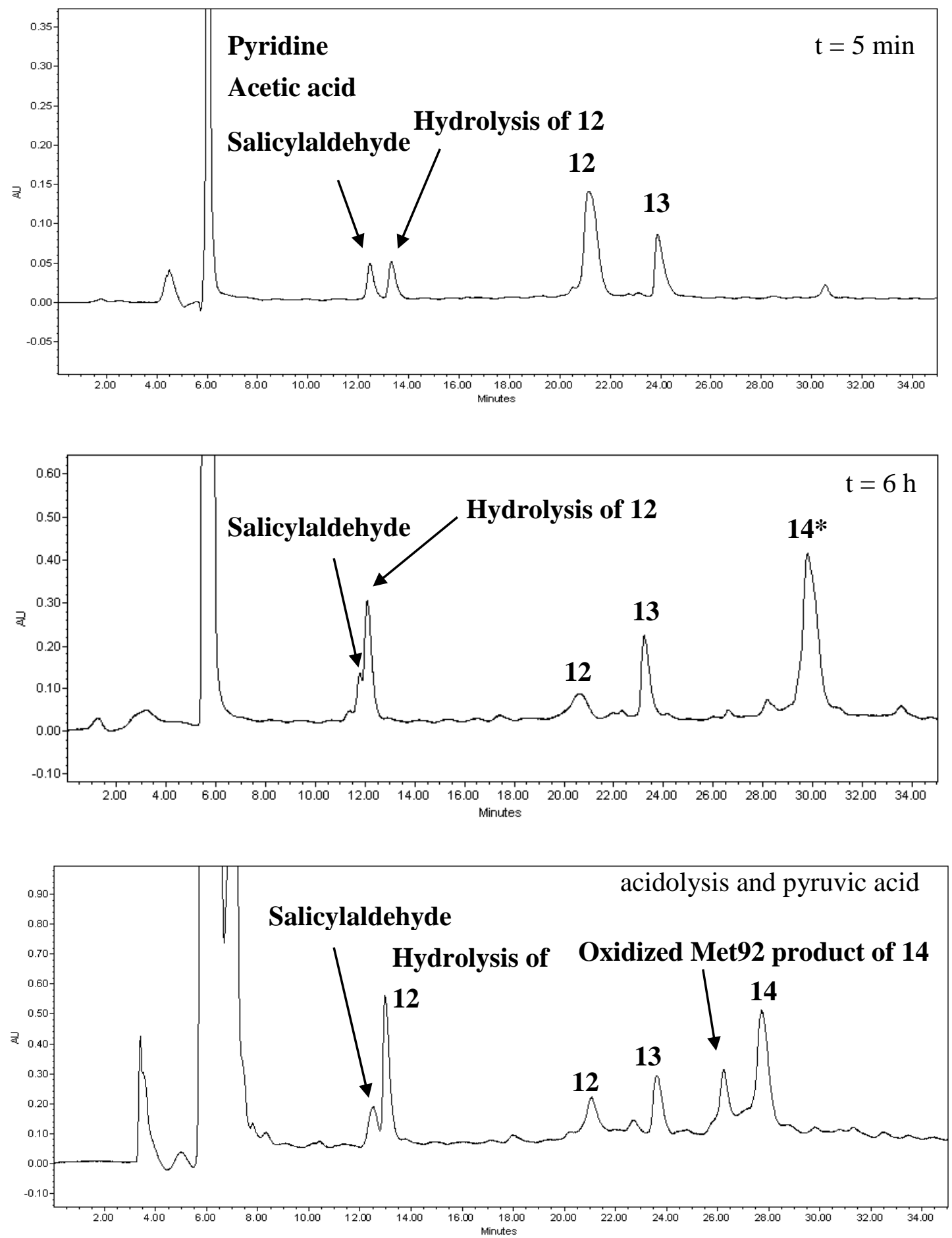

Figure S76. UV trace (190-400 nm) from LC-MS analysis of STL between 12 and $\mathbf{1 3}$ to give 14* at $5 \mathrm{~min}, 6 \mathrm{~h}$ and after in situ acidolysis and pyruvic acid to produce 14: gradient $25-35 \% \mathrm{CH}_{3} \mathrm{CN} / \mathrm{H}_{2} \mathrm{O}$ containing $0.1 \%$ TFA over $30 \mathrm{~min}$ at a flow rate of 0.6 $\mathrm{mL} / \mathrm{min}$. 


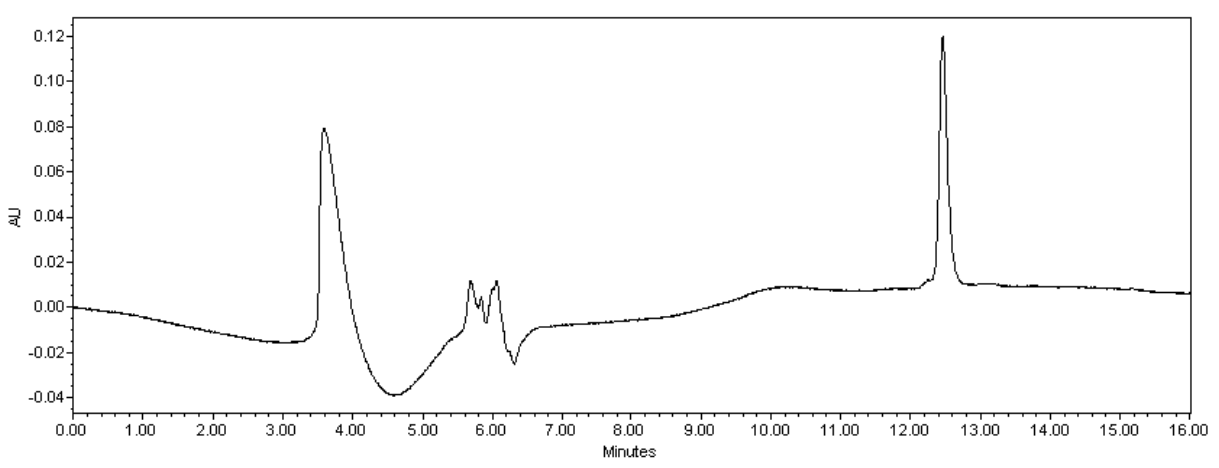

Figure S77. UV trace $(190-400 \mathrm{~nm})$ from LC-MS analysis of purified 14: gradient $5-95 \% \mathrm{CH}_{3} \mathrm{CN} / \mathrm{H}_{2} \mathrm{O}$ containing $0.1 \%$ TFA over $15 \mathrm{~min}$ at a flow rate of $0.6 \mathrm{~mL} / \mathrm{min}$.

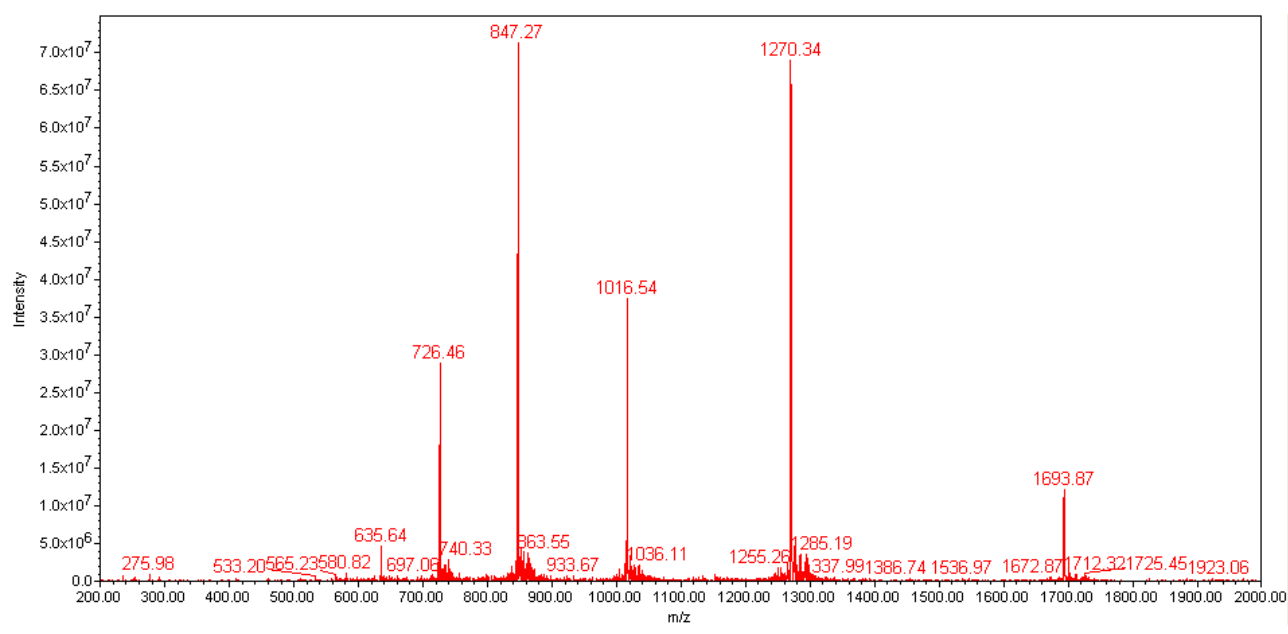

Figure S78. ESI-MS calcd. for $\mathrm{C}_{219} \mathrm{H}_{328} \mathrm{~N}_{62} \mathrm{O}_{66} \mathrm{~S}_{6}, 5077.71$ Da (average isotopes), $[\mathrm{M}+3 \mathrm{H}]^{3+} m / z=1693.57$, found 1693.87; $[\mathrm{M}+4 \mathrm{H}]^{4+} \mathrm{m} / \mathrm{z}=1270.43$, found 1270.34; $[\mathrm{M}+5 \mathrm{H}]^{5+} \mathrm{m} / \mathrm{z}=1016.54$, found 1016.54; $[\mathrm{M}+6 \mathrm{H}]^{6+} \mathrm{m} / \mathrm{z}=847.29$, found 847.27; $[\mathrm{M}+7 \mathrm{H}]^{7+} \mathrm{m} / \mathrm{z}=726.39$, found $726.46 ;[\mathrm{M}+8 \mathrm{H}]^{8+} \mathrm{m} / \mathrm{z}=635.71$, found 635.64 . 


\section{Glycopeptide fragment 18: IL-25 (80-145)}

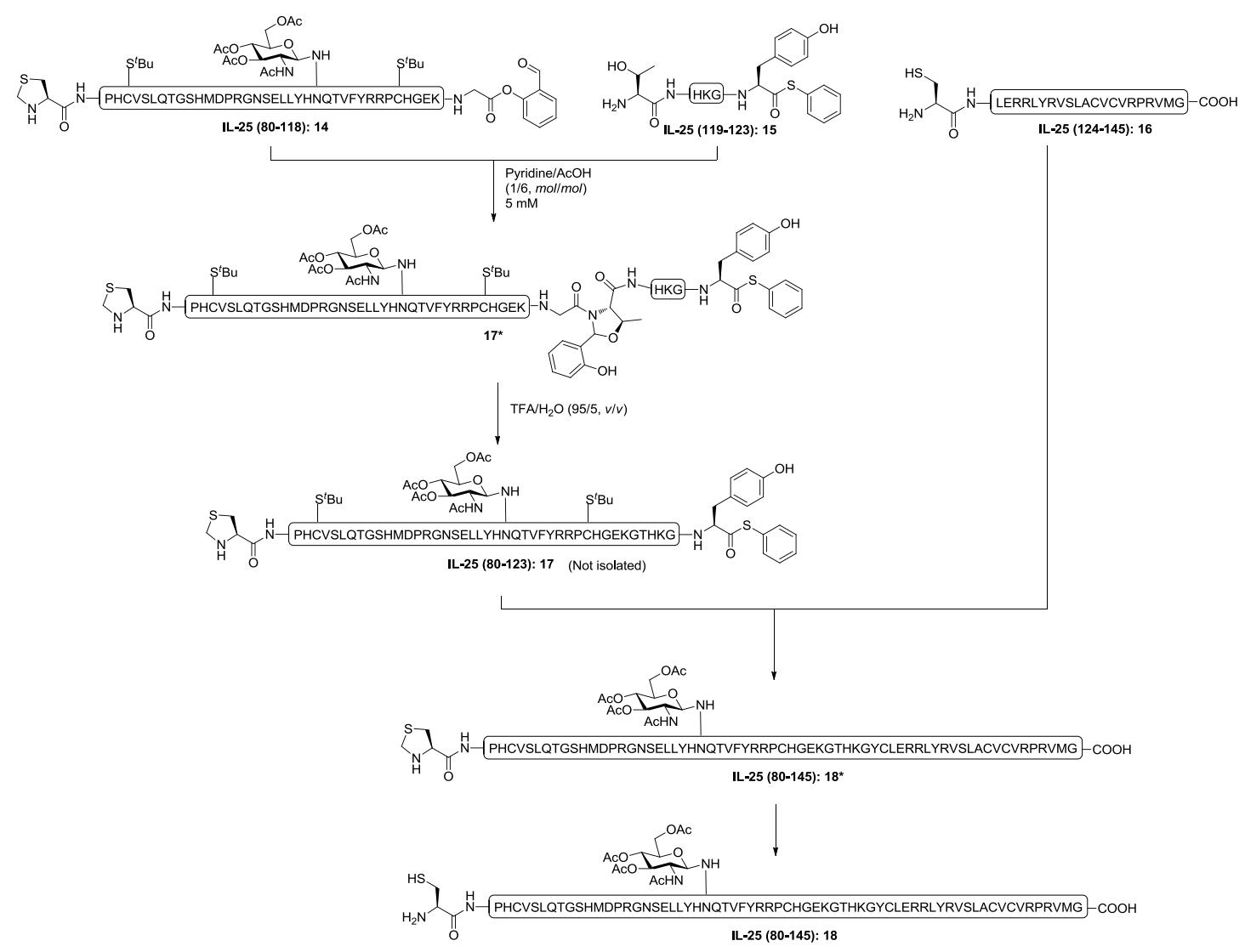

Scheme S15. One-pot Three-segment STL and NCL to produce (18).

$14(11.4 \mathrm{mg}, 2.2 \mu \mathrm{mol})$ and $\mathbf{1 5}(2.4 \mathrm{mg}, 3.4 \mu \mathrm{mol})$ were dissolved in pyridine/acetic acid $(1 / 6$, mole/mole) buffer at a concentration of $5 \mathrm{mM}$ at room temperature. The reaction mixture was stirred at room temperature for $4 \mathrm{~h}$ to give $17^{*}$, and the solvent was then blown off under a stream of condensed air. The residue was then treated with TFA/ $\mathrm{H}_{2} \mathrm{O}(95 / 5, v / v)$ for 5 min to obtain crude 17. Without purification, crude 17 and $16(17.3 \mathrm{mg}, 6.7 \mu \mathrm{mol})$ were dissolved in NCL buffer $(2 \mathrm{mM})$, prepared as described in general procedure E. The reaction mixture was stirred for $2 \mathrm{~h}$, and after completion of the ligation as assessed by HPLC, an aqueous solution of $\mathrm{MeONH}_{2} \cdot \mathrm{HCl}(74.8 \mu \mathrm{L}, 0.6 \mathrm{M})$ was added followed by TCEP solution $(62.4 \mu \mathrm{L}, 0.5$ $\mathrm{M})$. The $\mathrm{pH}$ was adjusted to $\sim 4.0-4.5$ by addition of $2 \mathrm{M} \mathrm{HCl}$ solution and the turbid mixture was stirred for $18 \mathrm{~h}$. After this time, the contents were purified by preparative HPLC $\left(15-35 \% \mathrm{CH}_{3} \mathrm{CN} / \mathrm{H}_{2} \mathrm{O}\right.$ over $\left.30 \mathrm{~min}\right)$ followed by concentration at reduced pressure and lyophilization afforded $3.6 \mathrm{mg}$ (20\% yield) of $\mathbf{1 8}$ as a white solid. 

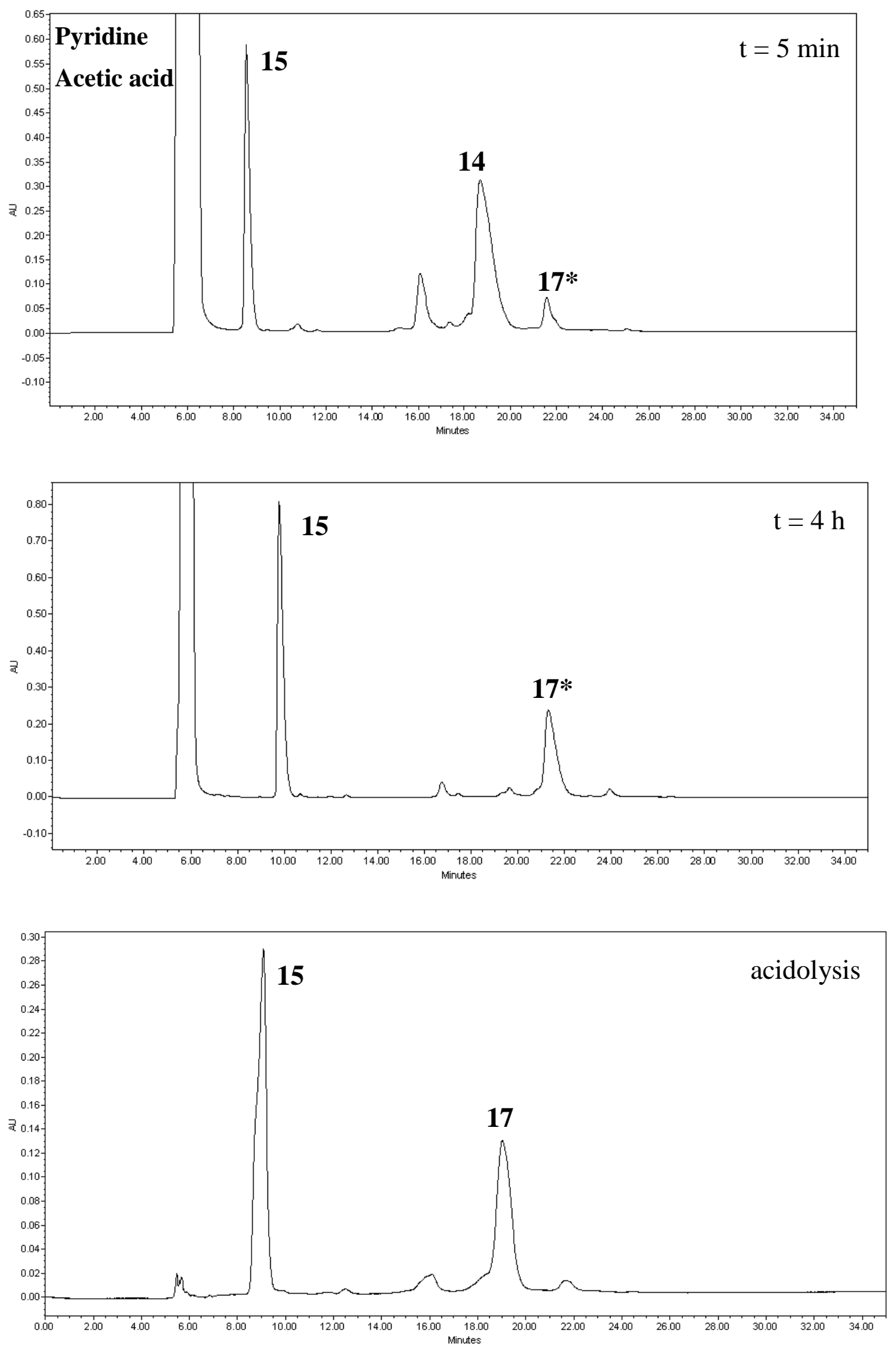

Figure S79. UV trace (190-400 nm) from LC-MS analysis of STL between 14 and 15 to generate $17^{*}$ at $\mathrm{t}=5 \mathrm{~min}, 4 \mathrm{~h}$ and after acidolysis to generate crude 17: gradient $25-40 \% \mathrm{CH}_{3} \mathrm{CN} / \mathrm{H}_{2} \mathrm{O}$ containing $0.1 \%$ TFA over $30 \mathrm{~min}$ at a flow rate of $0.6 \mathrm{~mL} / \mathrm{min}$. 

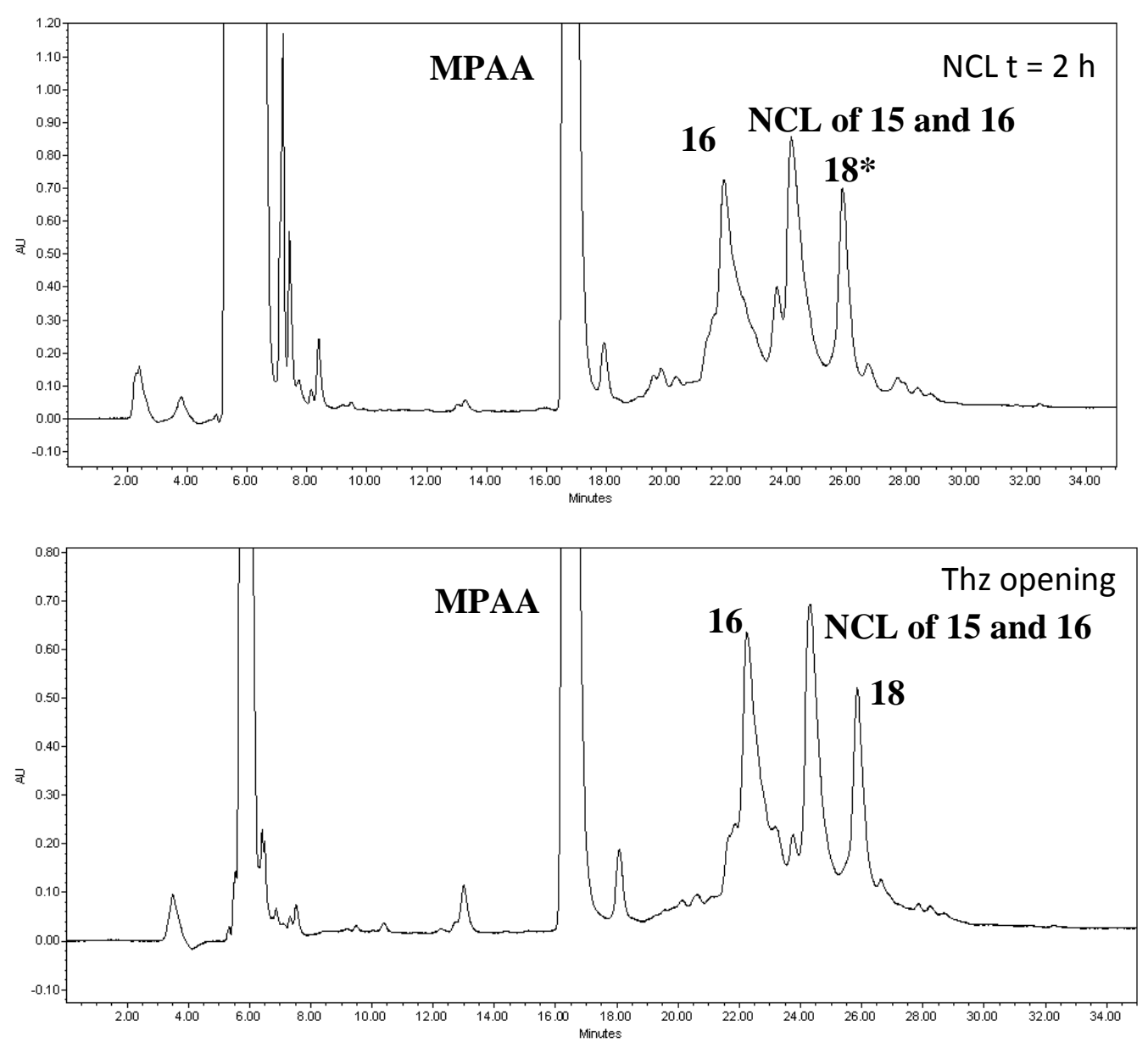

Figure S80. UV trace (190-400 nm) from LC-MS analysis of NCL between 16 and crude 17 to generate $18 *$ at $2 \mathrm{~h}$ and the conversion of $18 *$ to 18 (Thz opening): gradient $20-40 \% \mathrm{CH}_{3} \mathrm{CN} / \mathrm{H}_{2} \mathrm{O}$ containing $0.1 \%$ TFA over $30 \mathrm{~min}$ at a flow rate of 0.6 $\mathrm{mL} / \mathrm{min}$. 


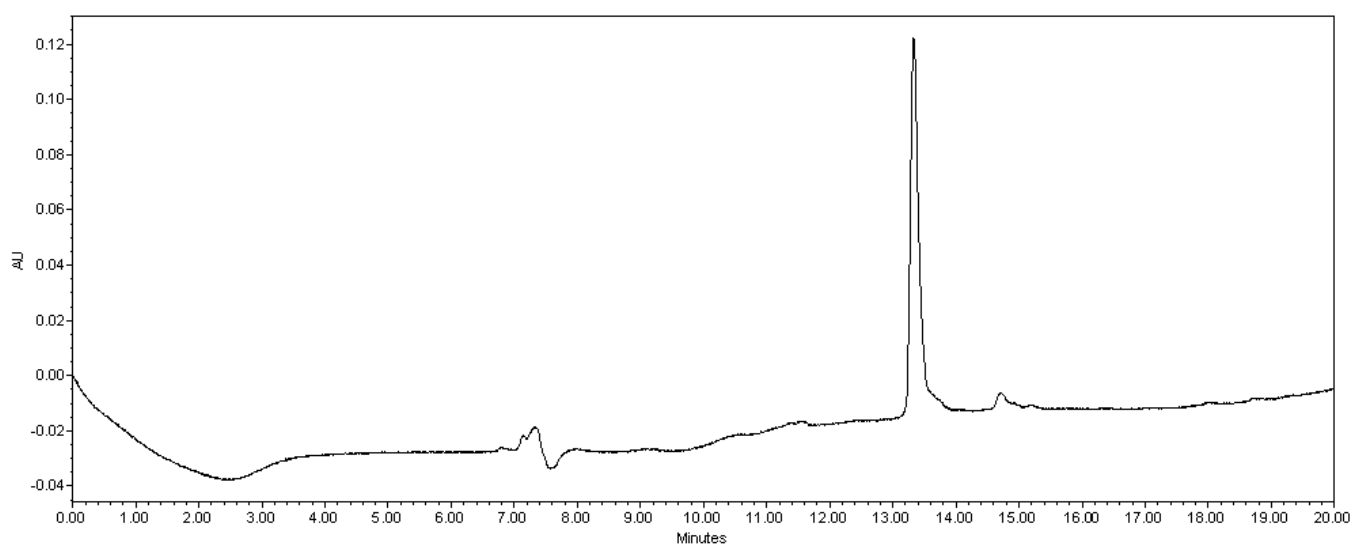

Figure S81. UV trace (190-400 nm) from LC-MS analysis of purified 18: gradient $5-95 \% \mathrm{CH}_{3} \mathrm{CN} / \mathrm{H}_{2} \mathrm{O}$ containing $0.1 \%$ TFA over $15 \mathrm{~min}$ at a flow rate of $0.6 \mathrm{~mL} / \mathrm{min}$.

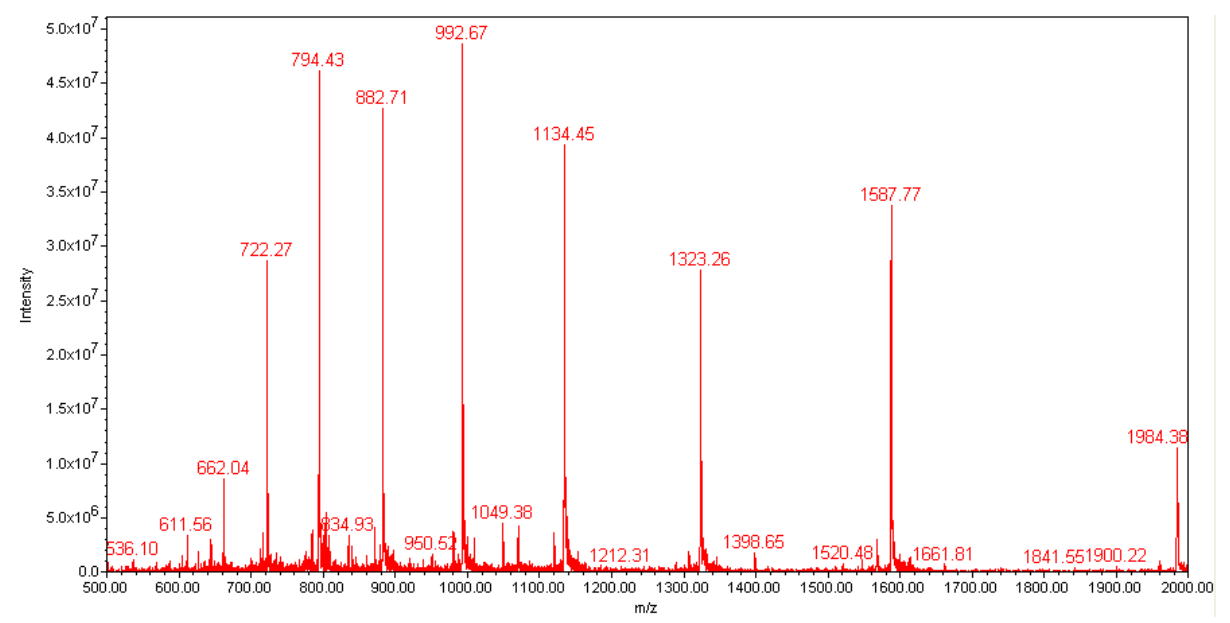

Figure S82. ESI-MS calcd. for $\mathrm{C}_{339} \mathrm{H}_{535} \mathrm{~N}_{107} \mathrm{O}_{98} \mathrm{~S}_{8}, 7934.05$ Da (average isotopes), $[\mathrm{M}+4 \mathrm{H}]^{4+} \mathrm{m} / \mathrm{z}=1984.51$, found 1984.38; $[\mathrm{M}+5 \mathrm{H}]^{5+} \mathrm{m} / \mathrm{z}=1587.81$, found 1587.77; $[\mathrm{M}+6 \mathrm{H}]^{6+} m / z=1323.34$, found 1323.26; $[\mathrm{M}+7 \mathrm{H}]^{7+} \mathrm{m} / z=1134.44$, found 1134.45; $[\mathrm{M}+8 \mathrm{H}]^{8+} \mathrm{m} / \mathrm{z}=992.77$, found 992.67; $[\mathrm{M}+9 \mathrm{H}]^{9+} \mathrm{m} / \mathrm{z}=882.56$, found 882.71; $[\mathrm{M}+10 \mathrm{H}]^{10+} \mathrm{m} / \mathrm{z}=794.41$, found $794.43 ;[\mathrm{M}+11 \mathrm{H}]^{11+} \mathrm{m} / \mathrm{z}=722.28$, found 722.27; $[\mathrm{M}+12 \mathrm{H}]^{12+} \mathrm{m} / \mathrm{z}=662.17$, found 662.04 . 


\section{Glycopeptide fragment 19: IL-25 (80-145)}

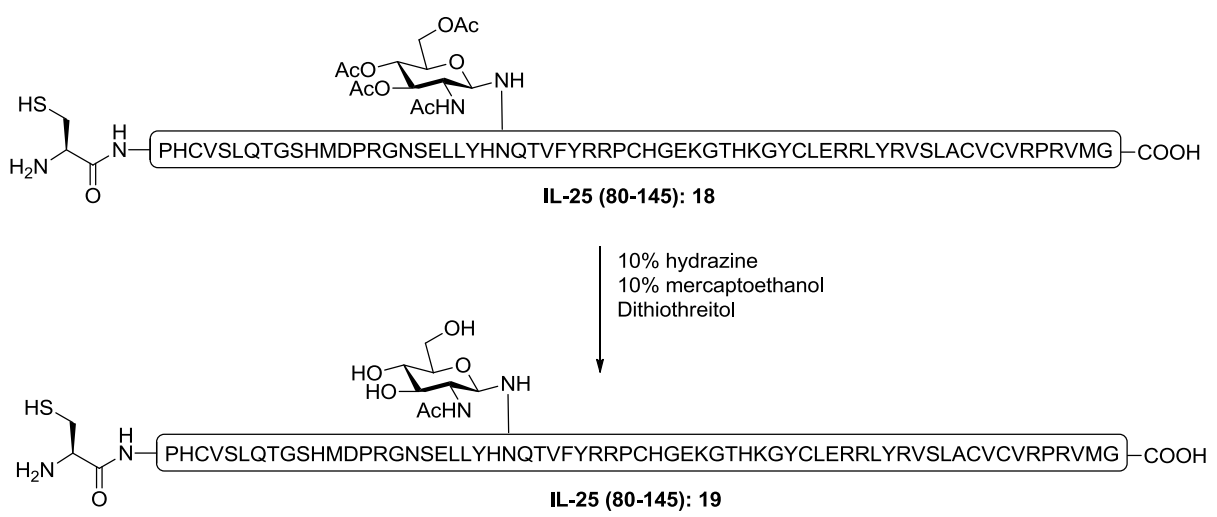

$18(3.6 \mathrm{mg}, \quad 0.4 \mu \mathrm{mol})$ was treated with $10 \%$ aqueous hydrazine, $10 \%$ $\beta$-mercaptoethanol, and dithiothreitol $(3.5 \mathrm{mg}, 22.7 \mu \mathrm{mol})(0.5 \mathrm{~mL}$, using hydrazine solution $64 \%$ wt in $\mathrm{H}_{2} \mathrm{O}$ ), and the solution was stirred for $2 \mathrm{~h}$. The reaction solution was quenched by aqueous tris(2-carboxyethyl)phosphine (TCEP) (10 mM) containing $6 \mathrm{M} \mathrm{Gn} \cdot \mathrm{HCl}(\mathrm{pH}=4.0)$ and the mixture was subjected to HPLC purification (20-35\% $\mathrm{CH}_{3} \mathrm{CN} / \mathrm{H}_{2} \mathrm{O}$ over $30 \mathrm{~min}$ ) followed by concentration at reduced pressure and lyophilization afforded $1.8 \mathrm{mg}$ (51\% yield) of $\mathbf{1 9}$ as a white solid.

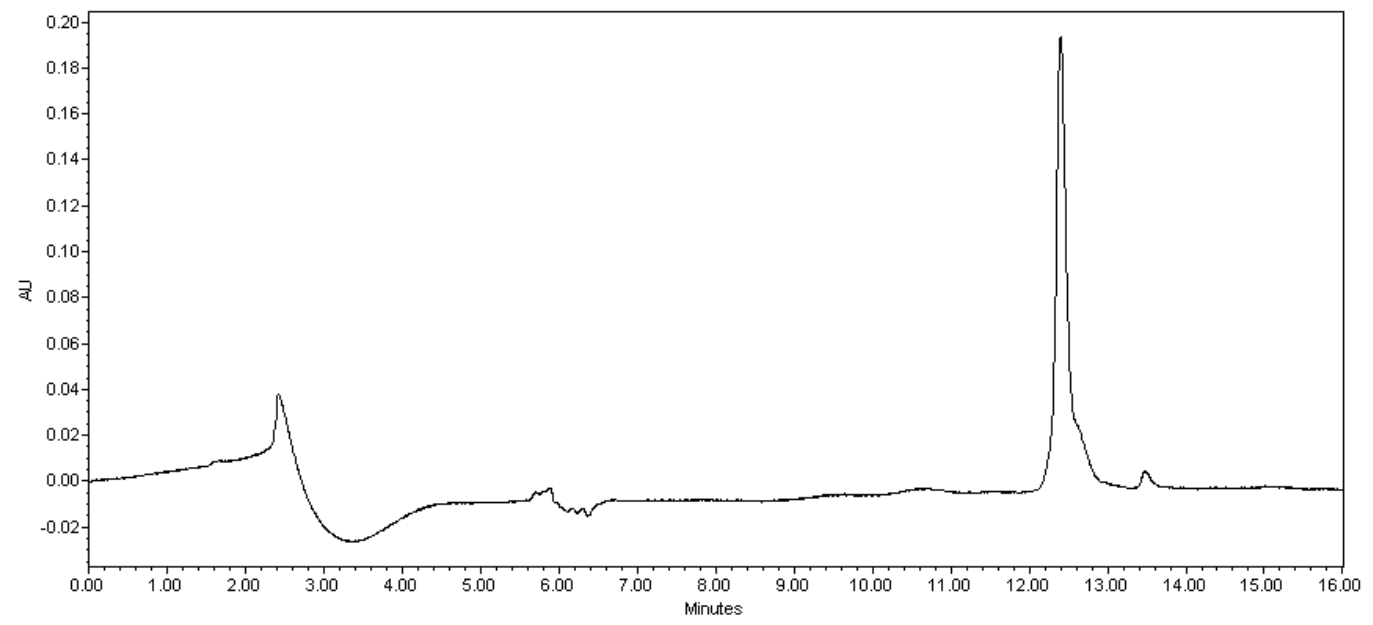

Figure S83. UV trace $(190-400 \mathrm{~nm})$ from LC-MS analysis of purified 19: gradient $5-95 \% \mathrm{CH}_{3} \mathrm{CN} / \mathrm{H}_{2} \mathrm{O}$ containing $0.1 \%$ TFA over $15 \mathrm{~min}$ at a flow rate of $0.6 \mathrm{~mL} / \mathrm{min}$. 


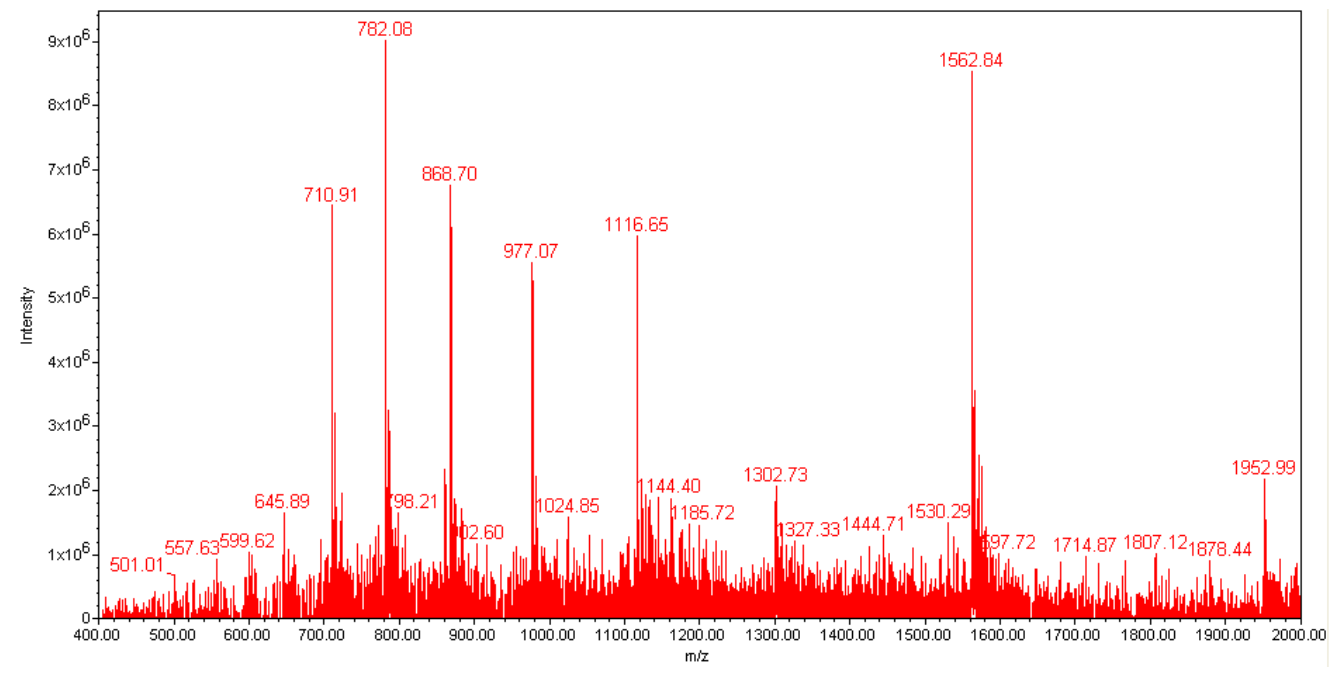

Figure S84. ESI-MS calcd. for $\mathrm{C}_{333} \mathrm{H}_{529} \mathrm{~N}_{107} \mathrm{O}_{95} \mathrm{~S}_{8}, 7807.94$ Da (average isotopes), $[\mathrm{M}+4 \mathrm{H}]^{4+} \mathrm{m} / z=1952.99$, found 1952.99; $[\mathrm{M}+5 \mathrm{H}]^{5+} \mathrm{m} / \mathrm{z}=1562.56$, found 1562.84; $[\mathrm{M}+6 \mathrm{H}]^{6+} \mathrm{m} / \mathrm{z}=1302.72$, found 1302.73; $[\mathrm{M}+7 \mathrm{H}]^{7+} \mathrm{m} / \mathrm{z}=1116.42$, found 1116.65; $[\mathrm{M}+8 \mathrm{H}]^{8+} \mathrm{m} / \mathrm{z}=976.99$, found 977.07; $[\mathrm{M}+9 \mathrm{H}]^{9+} \mathrm{m} / \mathrm{z}=868.55$, found 868.70; $[\mathrm{M}+10 \mathrm{H}]^{10+} \mathrm{m} / \mathrm{z}=781.79$, found $782.08 ;[\mathrm{M}+11 \mathrm{H}]^{11+} \mathrm{m} / \mathrm{z}=710.81$, found 710.91 . 
Peptide fragment 20: IL-25 (1-79) ${ }^{2}$

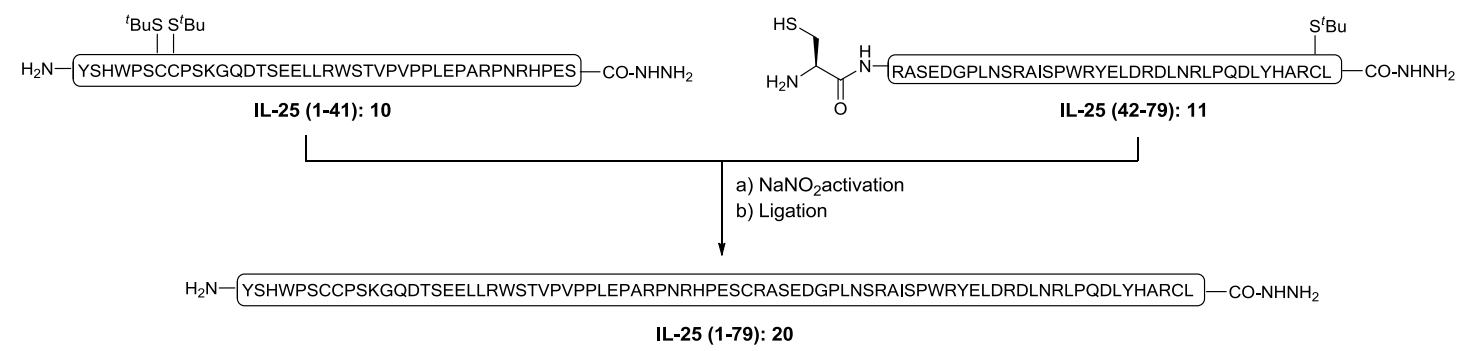

Scheme S16. Production of (20) by the hydrazide method.

Peptide 10 (15.1 mg, $3.1 \mu \mathrm{mol})$ was dissolved in an aqueous buffer containing $6 \mathrm{M}$ $\mathrm{Gn} \cdot \mathrm{HCl}$ and $0.2 \mathrm{M} \mathrm{NaH}_{2} \mathrm{PO}_{4}(\mathrm{pH}=3.0)$, and cooled to approximately $-11^{\circ} \mathrm{C}$ in an ice-salt bath. A solution of $\mathrm{NaNO}_{2}(2.14 \mathrm{mg}, 31.0 \mu \mathrm{mol})$ in the same buffer $(\mathrm{pH}=3.0)$ was then added dropwise to activate 10. After stirred at $-11^{\circ} \mathrm{C}$ for $15 \mathrm{~min}$, 4-mercaptophenylacetic acid (MPAA, $26.0 \mathrm{mg}, 154.8 \mu \mathrm{mol}$ ) was dissolved in $0.2 \mathrm{M}$ $\mathrm{NaH}_{2} \mathrm{PO}_{4}$ solution containing $6 \mathrm{M} \mathrm{Gn} \cdot \mathrm{HCl}(\mathrm{pH} 7.0$ ) was added into the mixture. The reaction was then taken out of the ice-salt bath and stirred at room temperature for 2 min. Subsequently, peptide $11(9.5 \mathrm{mg}, 2.1 \mu \mathrm{mol}$, final concentration $1 \mathrm{mM})$ was added into the reaction mixture. The $\mathrm{pH}$ value of reaction mixture was then adjusted to 7.0 slowly with aqueous $\mathrm{NaOH}$ solution $(1 \mathrm{M})$ to initiate the $\mathrm{NCL}$ at room temperature. The reaction solution was reduced by aqueous Tris(2-carboxyethyl)phosphine (TCEP) $(10 \mathrm{mM})$ containing $6 \mathrm{M} \mathrm{Gn} \cdot \mathrm{HCl}(\mathrm{pH}=4.0)$ with a quick operation before reaction analysis or product isolation. The reaction process was monitored by analytic RP-HPLC. After $6 \mathrm{~h}$, the ligation between $\mathbf{1 0}$ and 11 was completed. The product was purified by preparative HPLC (20-40\% $\mathrm{CH}_{3} \mathrm{CN} / \mathrm{H}_{2} \mathrm{O}$ over $30 \mathrm{~min}$,) followed by concentration at reduced pressure and lyophilization afforded $7.0 \mathrm{mg}$ (37\% yield) of $\mathbf{2 0}$ as a white solid. 

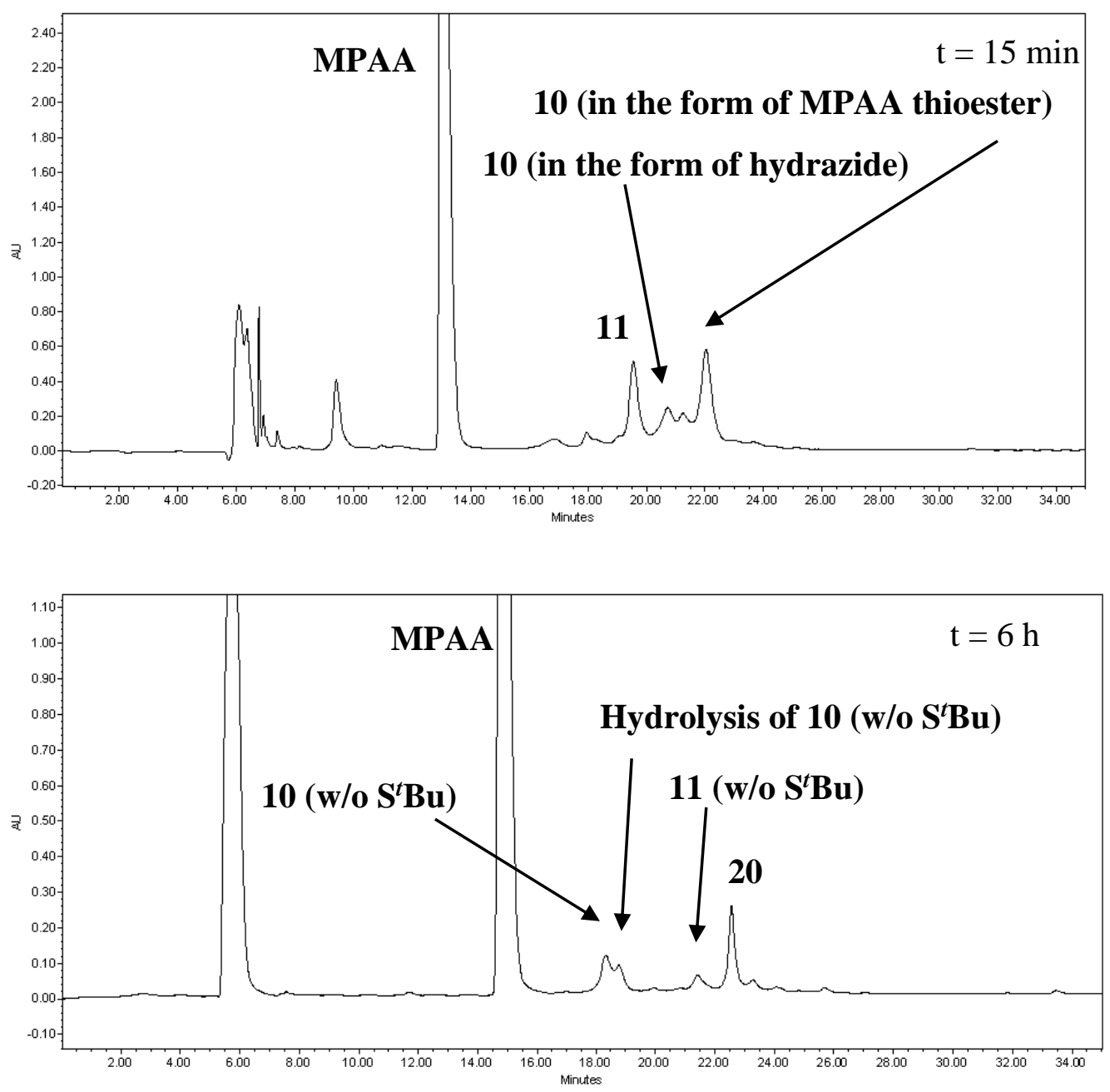

Figure S85. UV trace (190-400 nm) from LC-MS analysis of NCL between $\mathbf{1 0}$ and 11 to generate 20 at $\mathrm{t}=5 \mathrm{~min}$ and $6 \mathrm{~h}$ : gradient $25-50 \% \mathrm{CH}_{3} \mathrm{CN} / \mathrm{H}_{2} \mathrm{O}$ containing $0.1 \%$ TFA over $30 \mathrm{~min}$ at a flow rate of $0.6 \mathrm{~mL} / \mathrm{min}$.

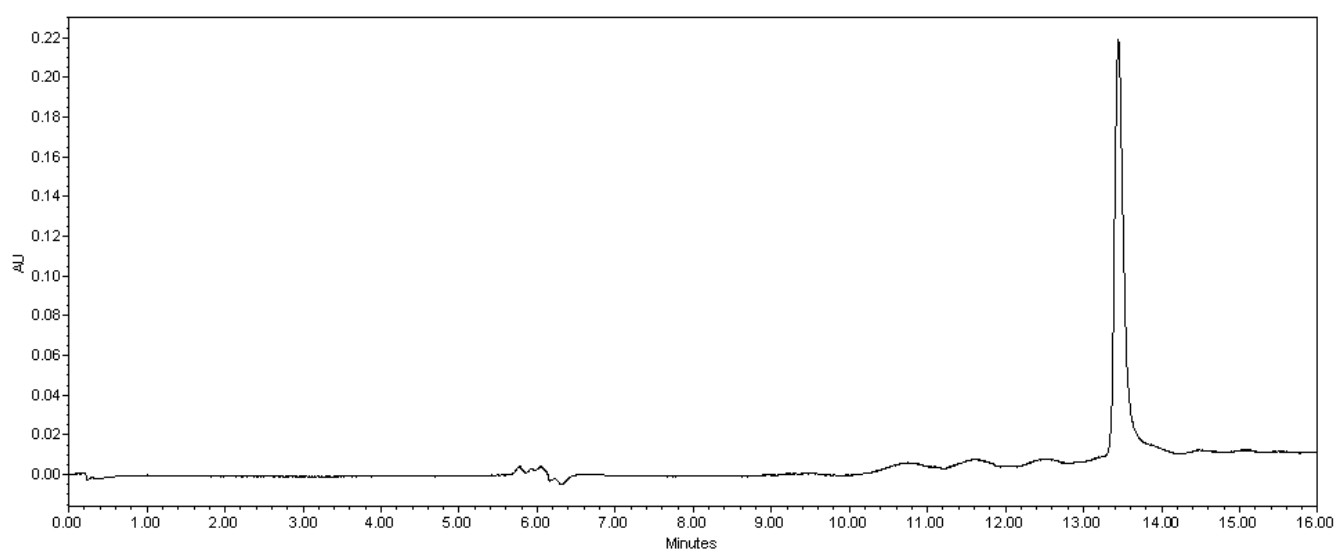

Figure S86. UV trace $(190-400 \mathrm{~nm})$ from LC-MS analysis of purified 20: gradient $5-95 \% \mathrm{CH}_{3} \mathrm{CN} / \mathrm{H}_{2} \mathrm{O}$ containing $0.1 \%$ TFA over $15 \mathrm{~min}$ at a flow rate of $0.6 \mathrm{~mL} / \mathrm{min}$. 


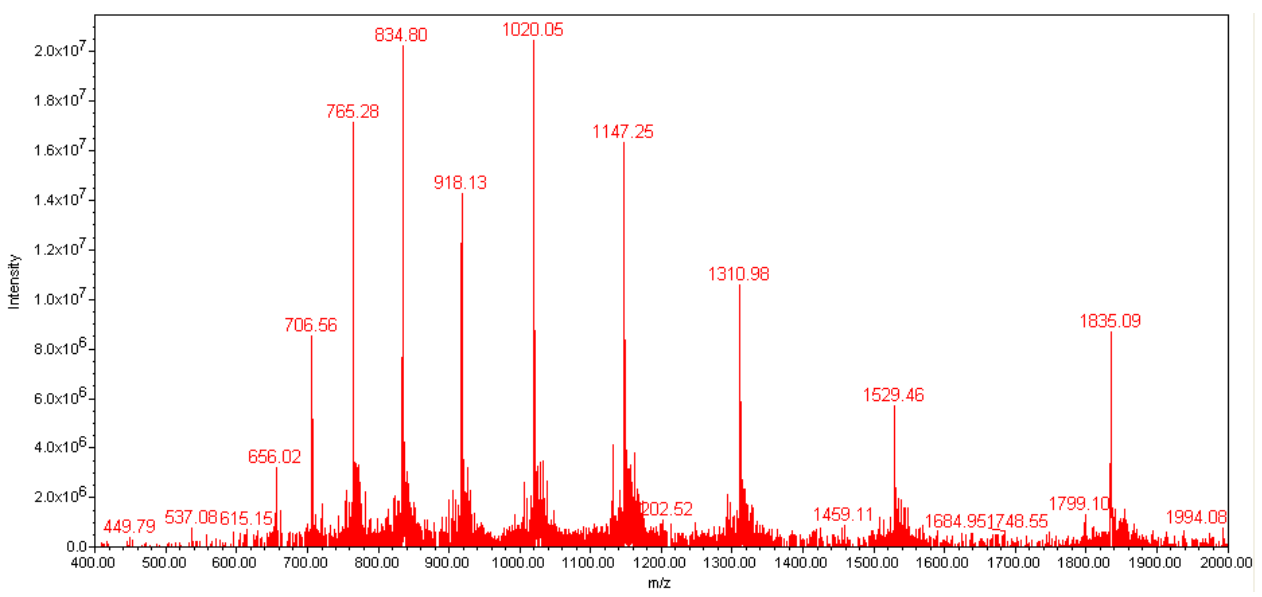

Figure S87. ESI-MS calcd. for $\mathrm{C}_{398} \mathrm{H}_{613} \mathrm{~N}_{123} \mathrm{O}_{120} \mathrm{~S}_{4}, 9169.14 \mathrm{Da}$ (average isotopes) $[\mathrm{M}+5 \mathrm{H}]^{5+} \mathrm{m} / \mathrm{z}=1834.83$, found 1835.09; $[\mathrm{M}+6 \mathrm{H}]^{6+} \mathrm{m} / \mathrm{z}=1529.19$, found 1529.46; $[\mathrm{M}+7 \mathrm{H}]^{7+} \mathrm{m} / z=1310.88$, found 1310.98; $[\mathrm{M}+8 \mathrm{H}]^{8+} \mathrm{m} / \mathrm{z}=1147.14$, found 1147.25; $[\mathrm{M}+9 \mathrm{H}]^{9+} \mathrm{m} / \mathrm{z}=1019.79$, found 1020.05; $[\mathrm{M}+10 \mathrm{H}]^{10+} \mathrm{m} / \mathrm{z}=917.91$, found 918.13; $[\mathrm{M}+11 \mathrm{H}]^{11+} \mathrm{m} / \mathrm{z}=834.56$, found 834.80; $[\mathrm{M}+12 \mathrm{H}]^{12+} \mathrm{m} / \mathrm{z}=765.10$, found 765.28; $[\mathrm{M}+13 \mathrm{H}]]^{13+} \mathrm{m} / z=706.32$, found $706.56 ;[\mathrm{M}+14 \mathrm{H}]^{14+} \mathrm{m} / \mathrm{z}=655.94$, found 656.02 . 


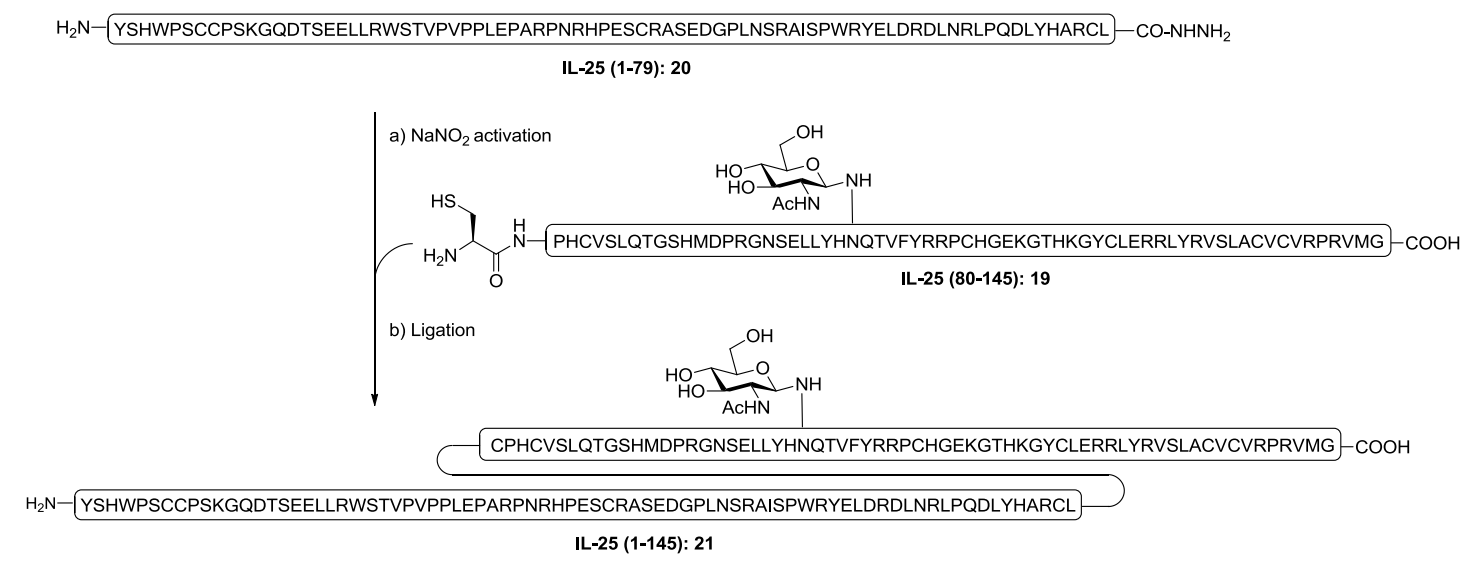

Scheme S17. Production of (21) by the hydrazide method.

Peptide 20 (2.1 mg, $0.2 \mu \mathrm{mol}$, final concentration $1 \mathrm{mM}$ ) was dissolved in an aqueous buffer containing $6 \mathrm{M} \mathrm{Gn} \cdot \mathrm{HCl}$ and $0.2 \mathrm{M} \mathrm{NaH}_{2} \mathrm{PO}_{4}(\mathrm{pH}=3.0)$, and cooled to approximately $-11{ }^{\circ} \mathrm{C}$ in an ice-salt bath. A solution of $\mathrm{NaNO}_{2}(15.8 \mu \mathrm{g}, 2.3 \mu \mathrm{mol})$ in the same buffer $(\mathrm{pH}=3.0)$ was then added dropwise to activate 20. After stirred at $-11^{\circ} \mathrm{C}$ for $15 \mathrm{~min}, 4-\mathrm{mercaptophenylacetic} \mathrm{acid}(2.9 \mathrm{mg}, 17.1 \mu \mathrm{mol})$ was dissolved in $0.2 \mathrm{M} \mathrm{Na}_{2} \mathrm{HPO}_{4}$ solution containing $6 \mathrm{M} \mathrm{Gn} \cdot \mathrm{HCl}(\mathrm{pH} 7.0)$ was added into the mixture. The reaction was then taken out of the ice-salt bath and stirred at room temperature for $2 \mathrm{~min}$. Subsequently, peptide $19(1.8 \mathrm{mg}, 0.2 \mu \mathrm{mol})$ was added into the reaction mixture. The $\mathrm{pH}$ value of reaction mixture was then adjusted to 7.2-7.4 slowly with aqueous $\mathrm{NaOH}$ solution $(1 \mathrm{M})$ to initiate the NCL at room temperature for $16 \mathrm{~h}$. The reaction solution was reduced by aqueous tris(2-carboxyethyl)phosphine (TCEP) (10 $\mathrm{mM})$ containing $6 \mathrm{M} \mathrm{Gn} \cdot \mathrm{HCl}(\mathrm{pH}=4.0)$ with a quick operation before reaction analysis or product isolation. The reaction process was monitored by analytic RP-HPLC. After $16 \mathrm{~h}$, the ligation between 19 and 20 was completed. The product was purified by preparative HPLC $\left(25-40 \% \mathrm{CH}_{3} \mathrm{CN} / \mathrm{H}_{2} \mathrm{O}\right.$ over $\left.30 \mathrm{~min}\right)$ followed by concentration at reduced pressure and lyophilization afforded $1.3 \mathrm{mg}$ (32\% yield) of 21 as a white solid. 

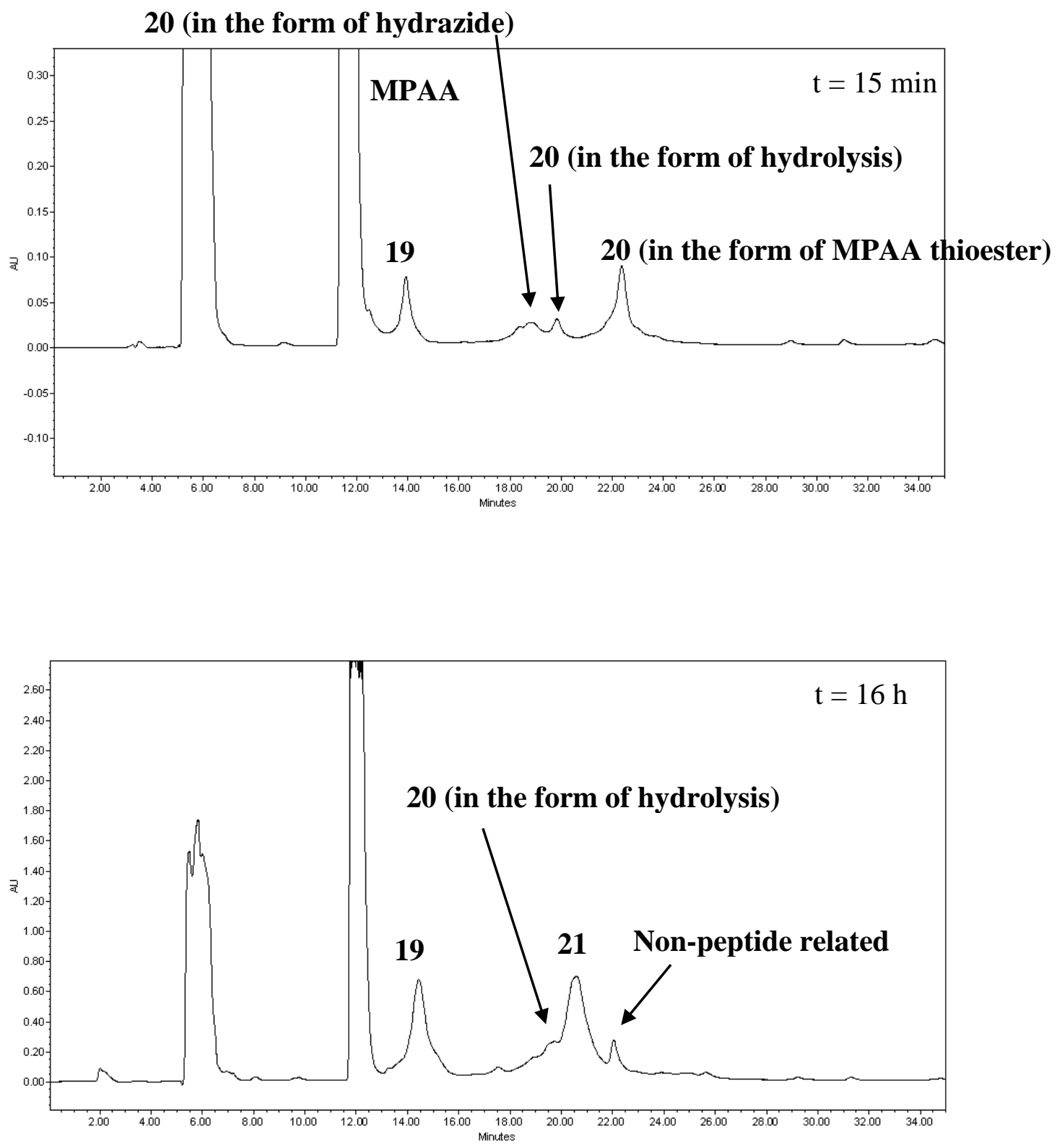

Figure S88. UV trace (190-400 nm) from LC-MS analysis of NCL between 19 and 20 to generate 21 at $\mathrm{t}=5 \mathrm{~min}$ and $16 \mathrm{~h}$ : gradient $30-50 \% \mathrm{CH}_{3} \mathrm{CN} / \mathrm{H}_{2} \mathrm{O}$ containing $0.1 \%$ TFA over $30 \mathrm{~min}$ at a flow rate of $0.6 \mathrm{~mL} / \mathrm{min}$. 


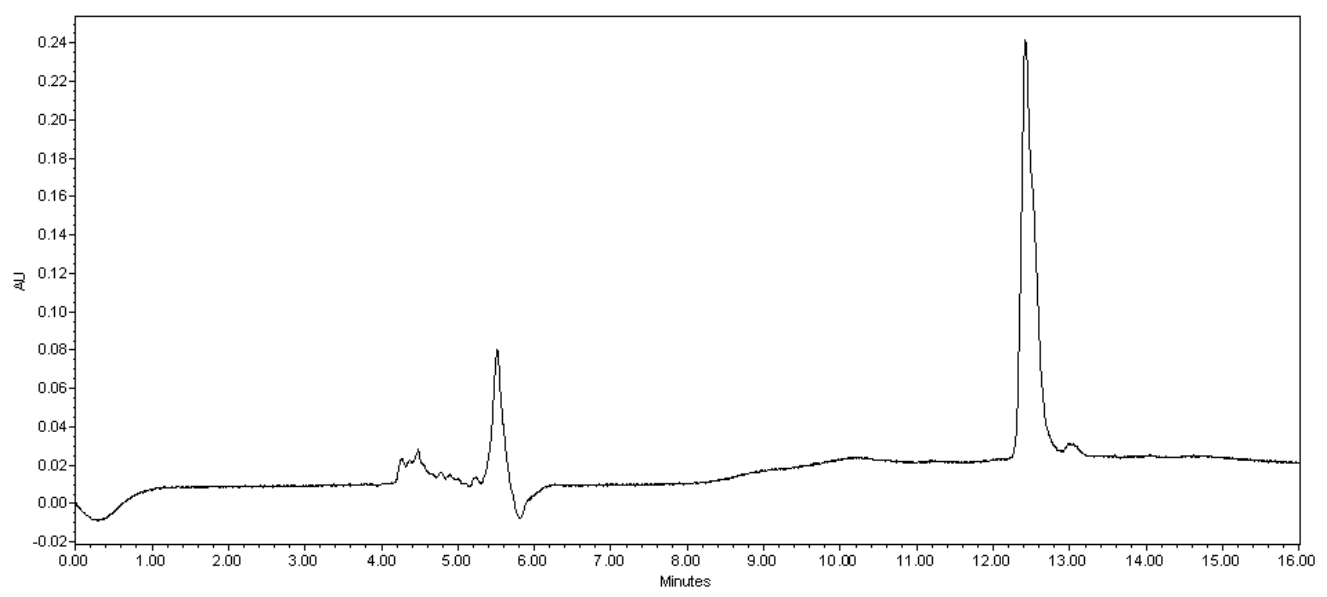

Figure S89. UV trace $(190-400 \mathrm{~nm})$ from LC-MS analysis of purified 21: gradient $5-95 \% \mathrm{CH}_{3} \mathrm{CN} / \mathrm{H}_{2} \mathrm{O}$ containing $0.1 \%$ TFA over $15 \mathrm{~min}$ at a flow rate of $0.6 \mathrm{~mL} / \mathrm{min}$.

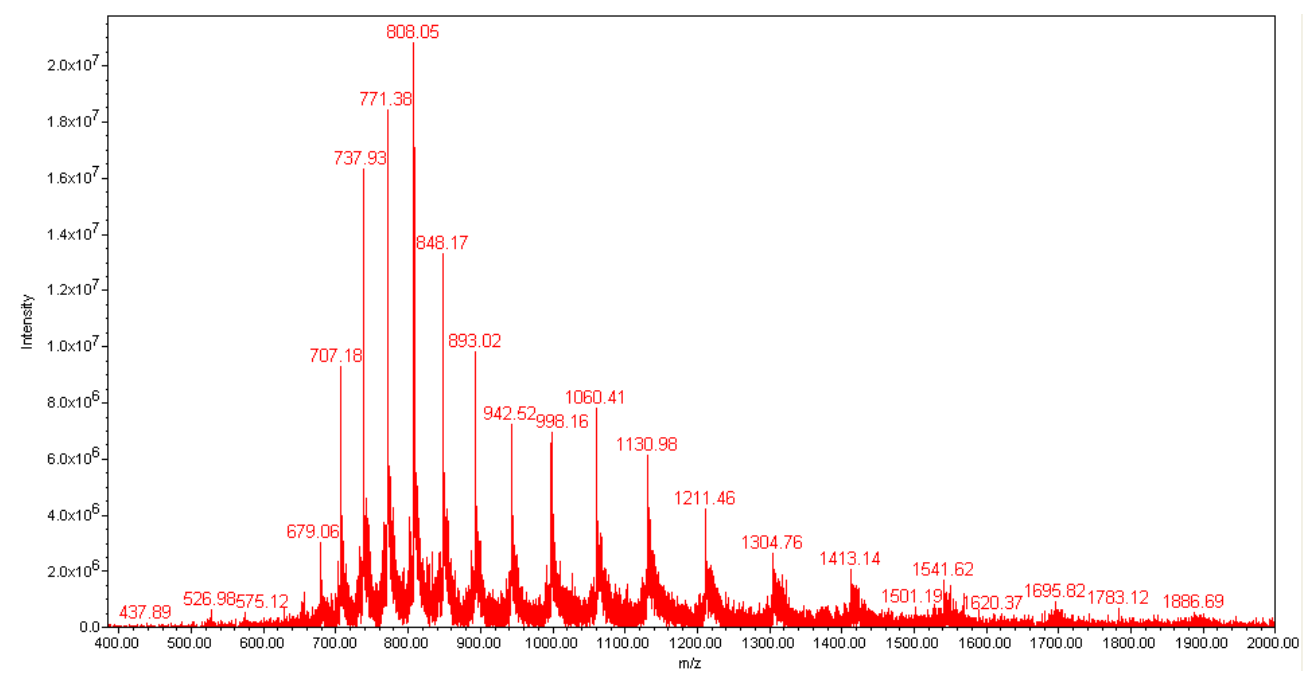

Figure S90. ESI-MS calcd. for $\mathrm{C}_{731} \mathrm{H}_{1138} \mathrm{~N}_{228} \mathrm{O}_{215} \mathrm{~S}_{12}, 16945.04 \mathrm{Da}$ (average isotopes), $[\mathrm{M}+10 \mathrm{H}]^{10+} \mathrm{m} / \mathrm{z}=1695.50$, found $1695.82 ;[\mathrm{M}+11 \mathrm{H}]^{11+} \mathrm{m} / \mathrm{z}=1541.46$, found 1541.62; $[\mathrm{M}+12 \mathrm{H}]^{12+} \mathrm{m} / \mathrm{z}=1413.09$, found 1413.14; $[\mathrm{M}+13 \mathrm{H}]^{13+} \mathrm{m} / \mathrm{z}=1304.47$, found 1304.76; $[\mathrm{M}+14 \mathrm{H}]^{14+} \mathrm{m} / \mathrm{z}=1211.36$, found 1211.46; $[\mathrm{M}+15 \mathrm{H}]^{15+} \mathrm{m} / \mathrm{z}=$ 1130.67, found 1130.98; $[\mathrm{M}+16 \mathrm{H}]^{16+} \mathrm{m} / \mathrm{z}=1060.07$, found $1060.41 ;[\mathrm{M}+17 \mathrm{H}]^{17+} \mathrm{m} / \mathrm{z}$ $=997.77$, found 998.16; $[\mathrm{M}+18 \mathrm{H}]^{18+} \mathrm{m} / \mathrm{z}=942.39$, found $942.52 ;[\mathrm{M}+19 \mathrm{H}]^{19+} \mathrm{m} / \mathrm{z}=$ 892.84, found 893.02; $[\mathrm{M}+20 \mathrm{H}]^{20+} \mathrm{m} / \mathrm{z}=848.25$, found $848.17 ;[\mathrm{M}+21 \mathrm{H}]^{21+} \mathrm{m} / z=$ 807.91, found 808.05; $[\mathrm{M}+22 \mathrm{H}]^{22+} \mathrm{m} / \mathrm{z}=771.23$, found $771.38 ;[\mathrm{M}+23 \mathrm{H}]^{23+} \mathrm{m} / \mathrm{z}=$ 737.74, found 737.93; $[\mathrm{M}+24 \mathrm{H}]^{24+} \mathrm{m} / z=707.04$, found $707.18 ;[\mathrm{M}+25 \mathrm{H}]^{25+} \mathrm{m} / \mathrm{z}=$ 678.80 , found 679.06 . 


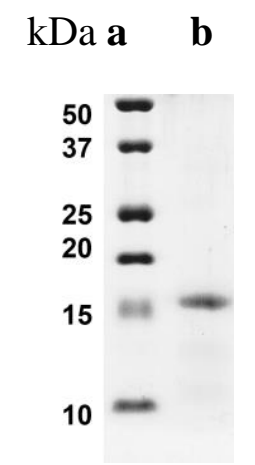

Figure S91. SDS-PAGE analysis of 21. Lane a: marker; lane b: IL-25 (1-145) 21.

\section{Folding of IL-25 glycopeptide (21)}

Several folding conditions were tried to perform oxidative folding of $\mathbf{2 1}$.

1) Oxidative folding of 21 was carried out, by following Rao's condition ${ }^{3}$, at $4^{\circ} \mathrm{C}$ for 6 hours in the folding buffer of $25 \mathrm{mM}$ Tris $\cdot \mathrm{HCl}, 200 \mathrm{mM} \mathrm{NaCl}, 10 \%$ glycerol, $1 \mathrm{mM}$ GSH, $10 \mathrm{mM}$ GSSG, $0.5 \mathrm{M}$ arginine and $2 \mathrm{mM}$ EDTA at a concentration of $1 \mathrm{mg} / \mathrm{mL}$ at $\mathrm{pH}$ 7.2. LCMS analysis showed that no desired folded protein was formed. 2) By using Unverzagt's condition ${ }^{4}, \mathbf{2 1}$ was then incubated in buffer of $0.1 \mathrm{M}$ Tris, $2 \mathrm{mM}$ EDTA, 0.3 mM GSSG at pH 7.7 for 4 days. However, this did not work in our case as suggested by LCMS. 3) Following another procedure published by Unverzagt's group $^{5}, 21$ was then dissolved in buffer containing $6 \mathrm{M} \mathrm{Gn} \cdot \mathrm{HCl}, 0.1 \mathrm{M} \mathrm{Na}_{2} \mathrm{HPO}_{4}, 0.1$ M MPAA, $5 \mathrm{mM}$ TCEP at $\mathrm{pH}$ 7. The buffer was then diluted to refolding buffer of 0.5 $\mathrm{M}$ arginine, $150 \mathrm{mM} \mathrm{NaCl}, 50 \mathrm{mM} \mathrm{Na} 2 \mathrm{HPO}_{4}, \mathrm{pH}$ 7.4. It was oxidized in the open tube for 4 days. However, LCMS analysis showed no desired folded product. 4) According to Liu's folding condition ${ }^{6}, \mathbf{2 1}$ was subjected to Tris. $\mathrm{HCl}$ buffer containing $1 \mathrm{M} \mathrm{Gn} \cdot \mathrm{HCl}, 10 \%(v / v)$ DMSO at $\mathrm{pH} 8.5$ for 24 hours. However, the result for 21 folding was failed. 5) Finally, dialysis was adopted following Kajihara's condition ${ }^{7}$. 21 was dissolved in a Tris $\cdot \mathrm{HCl}$ buffer $(100 \mathrm{mM}, \mathrm{pH} 7.5)$ containing $6 \mathrm{M} \mathrm{Gn} \cdot \mathrm{HCl}$ at concentration of $0.1 \mathrm{mg} / \mathrm{mL}$. This solution was poured into the dialysis tubing (MWCO at 3,000) and then dialyzed against the first folding buffer (3 M Gn-HCl, 100 $\mathrm{mM}$ Tris $\mathrm{HCl}, \mathrm{pH} 8.5$ ) containing $4 \mu \mathrm{M}$ GSH and $0.5 \mu \mathrm{M}$ GSSG for redox system and left for 12 hours at $4^{\circ} \mathrm{C}$. Then, the external buffer solution was replaced the second 
folding buffer solution (1 M Gn· $\mathrm{HCl}, 100 \mathrm{mM}$ Tris $\cdot \mathrm{HCl}, \mathrm{pH} 8.0)$ and dialysis was performed for 8 hours. Finally, the external buffer was discarded and changed again to the third folding buffer solution (10 $\mathrm{mM}$ Tris $\mathrm{HCl}, \mathrm{pH} 7.0)$ and dialysis was performed for 24 hours. Nonetheless, there is no good results as precipitate was formed. LCMS suggested no correctly folded IL-25 was observed. 


\section{$\underline{\text { XI. }{ }^{1} \mathrm{H} \text { and }{ }^{13} \mathrm{C} \text { NMR spectra }}$}

\section{$\underline{{ }^{1} \mathrm{H} \text { spectrum of } \mathrm{BocH} N-\operatorname{Ser}\left(\mathrm{O}^{t} \mathrm{Bu}\right)-\mathrm{CO}-\mathrm{SAL}^{\text {off }}}$<smiles>CC(C)(C)OCC(NC(=O)OC(C)(C)C)C(=O)Oc1ccccc1C=NNC(N)=O</smiles>
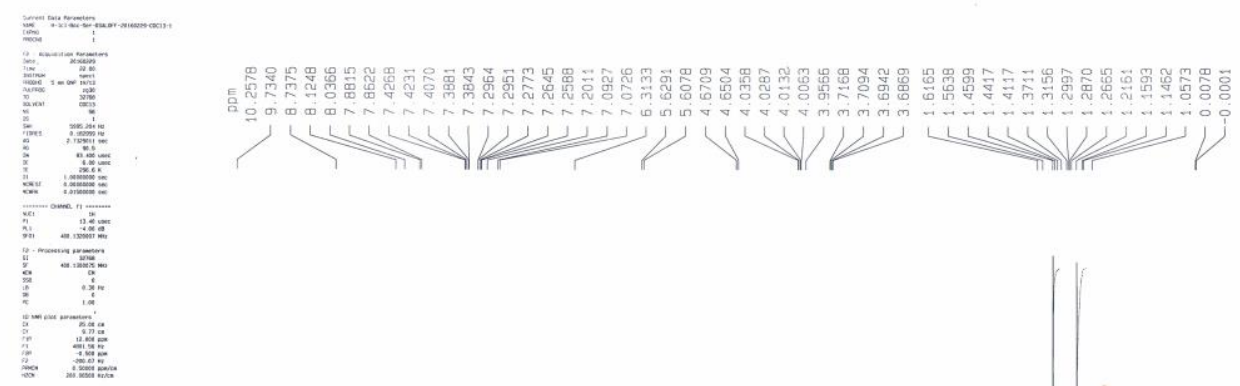
${ }^{13} \mathrm{C}$ spectrum of $\mathrm{BocHN}-\mathrm{Ser}\left(\mathrm{O}^{t} \mathrm{Bu}\right)-C O-\mathrm{SAL} \mathrm{L}^{\text {ff }}$
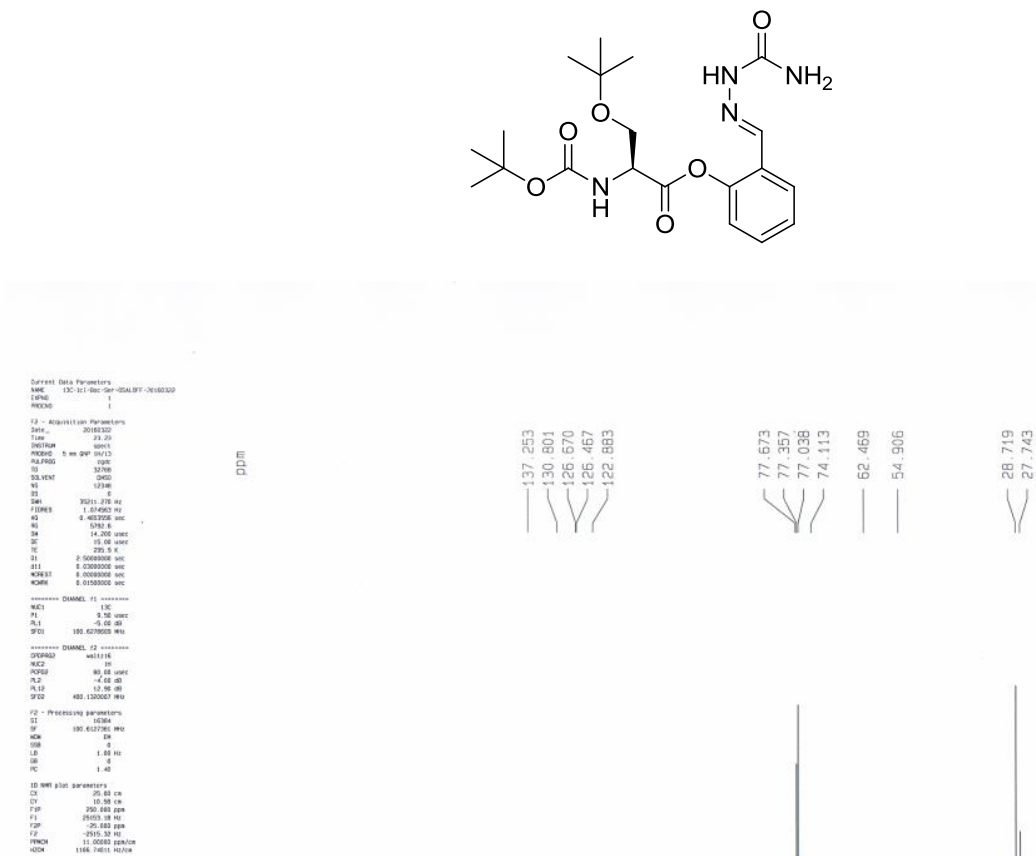

ar 
$\underline{{ }^{1} \mathrm{H} \text { spectrum of } \mathrm{BocHN} \text {-Ala-CO-SAL }{ }^{\text {off }}}$
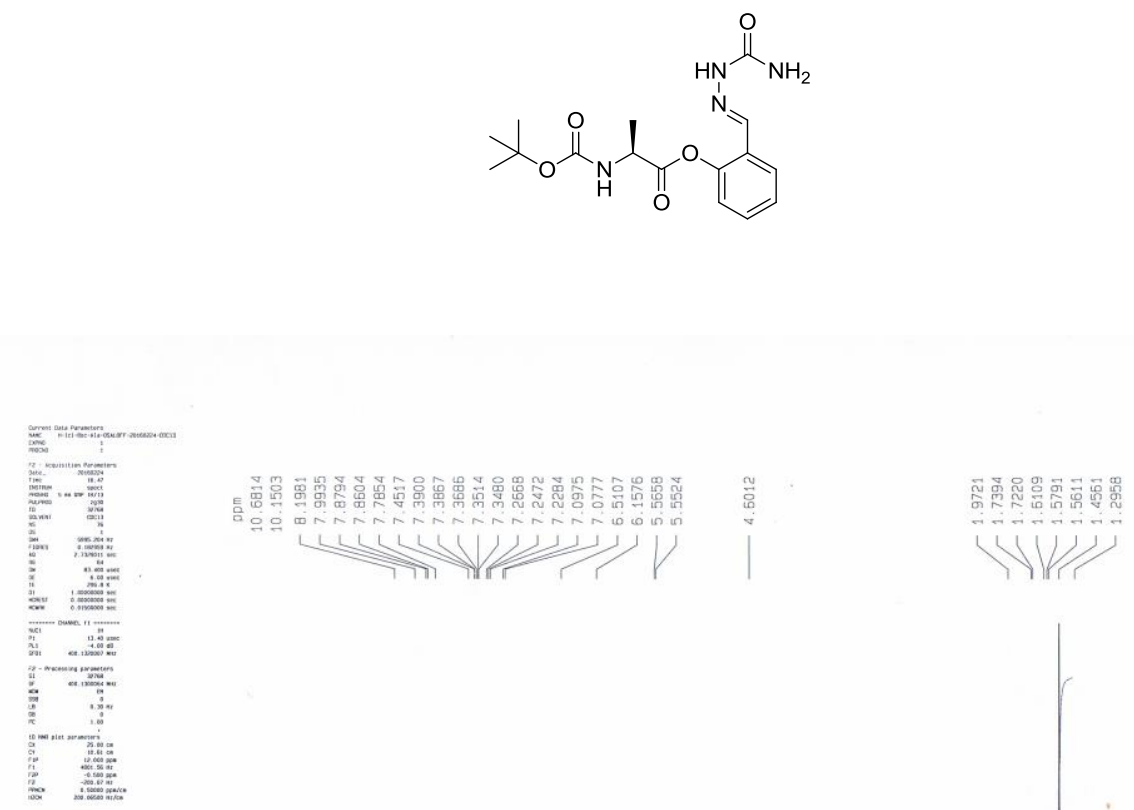

i
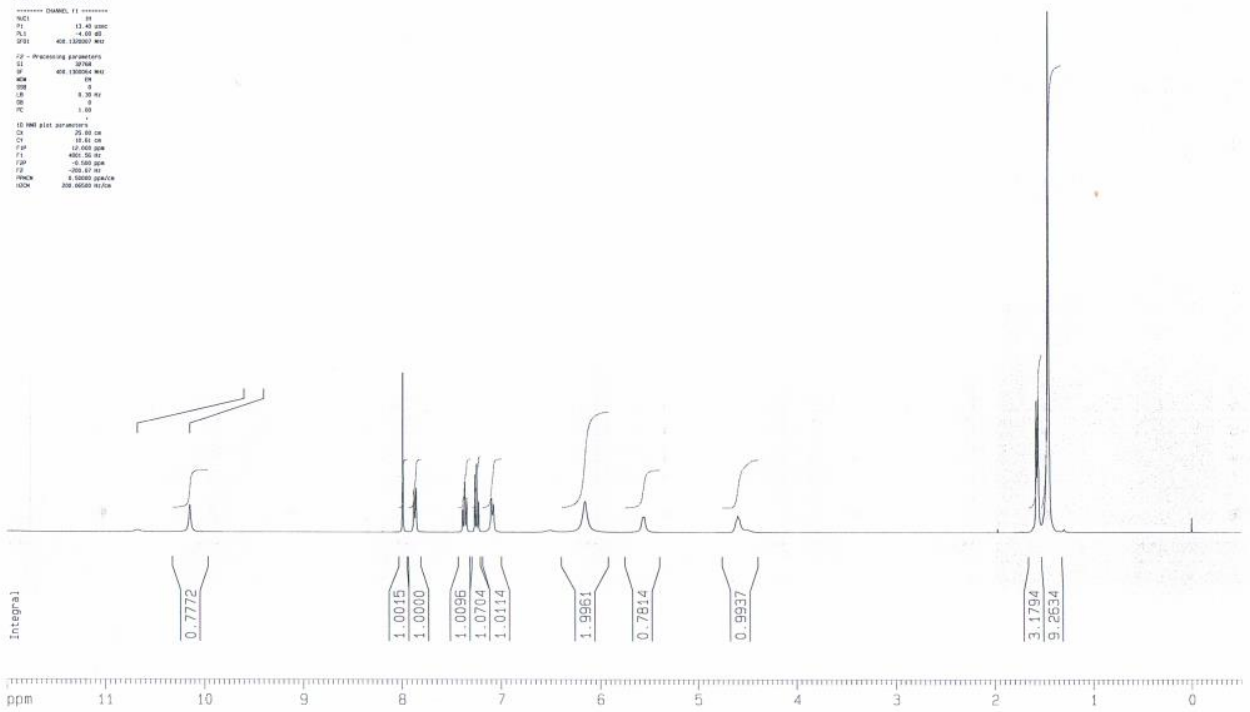

mim

S96 
${ }^{13} \mathrm{C}$ spectrum of $\mathrm{BocHN}$-Ala-CO-SAL ${ }^{\text {off }}$
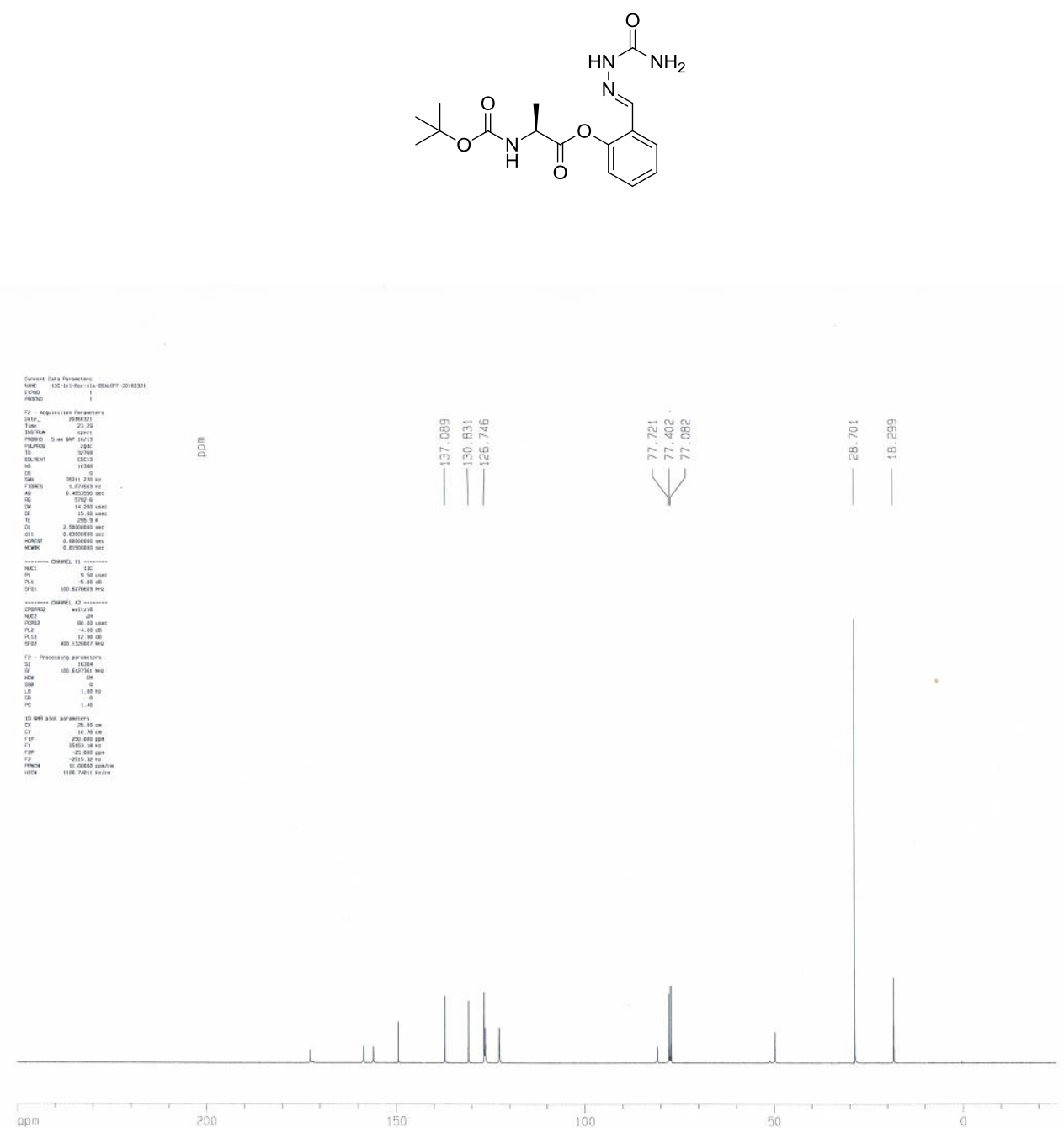

S97 


\section{${ }^{1} \mathrm{H}$ spectrum of $\mathrm{BocHN}$-Gly-CO-SAL ${ }^{\text {off }}$}
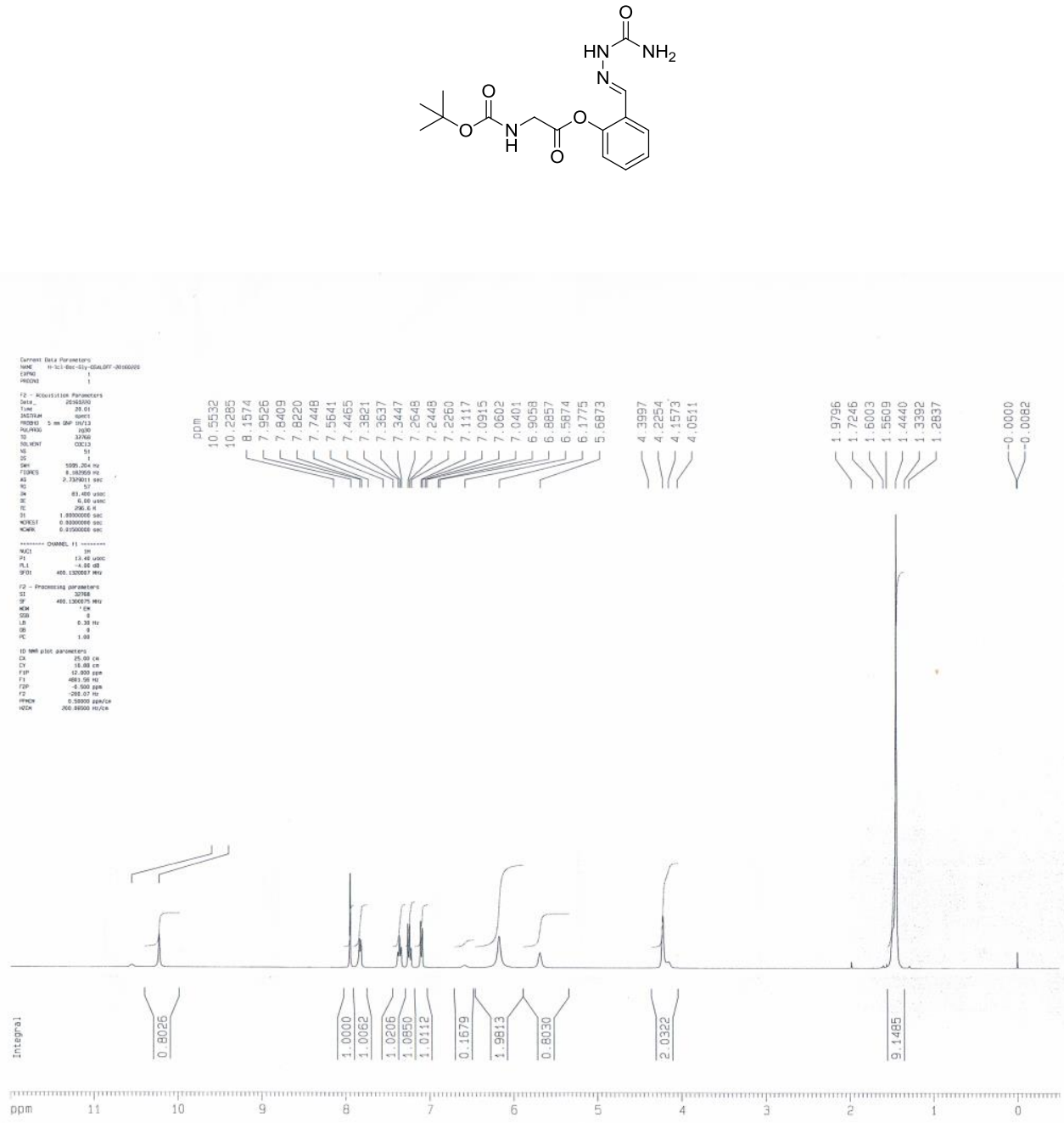
${ }^{13} \mathrm{C}$ spectrum of $\mathrm{BocHN}-\mathrm{Gly}-\mathrm{CO}-\mathrm{SAL}{ }^{\text {off }}$
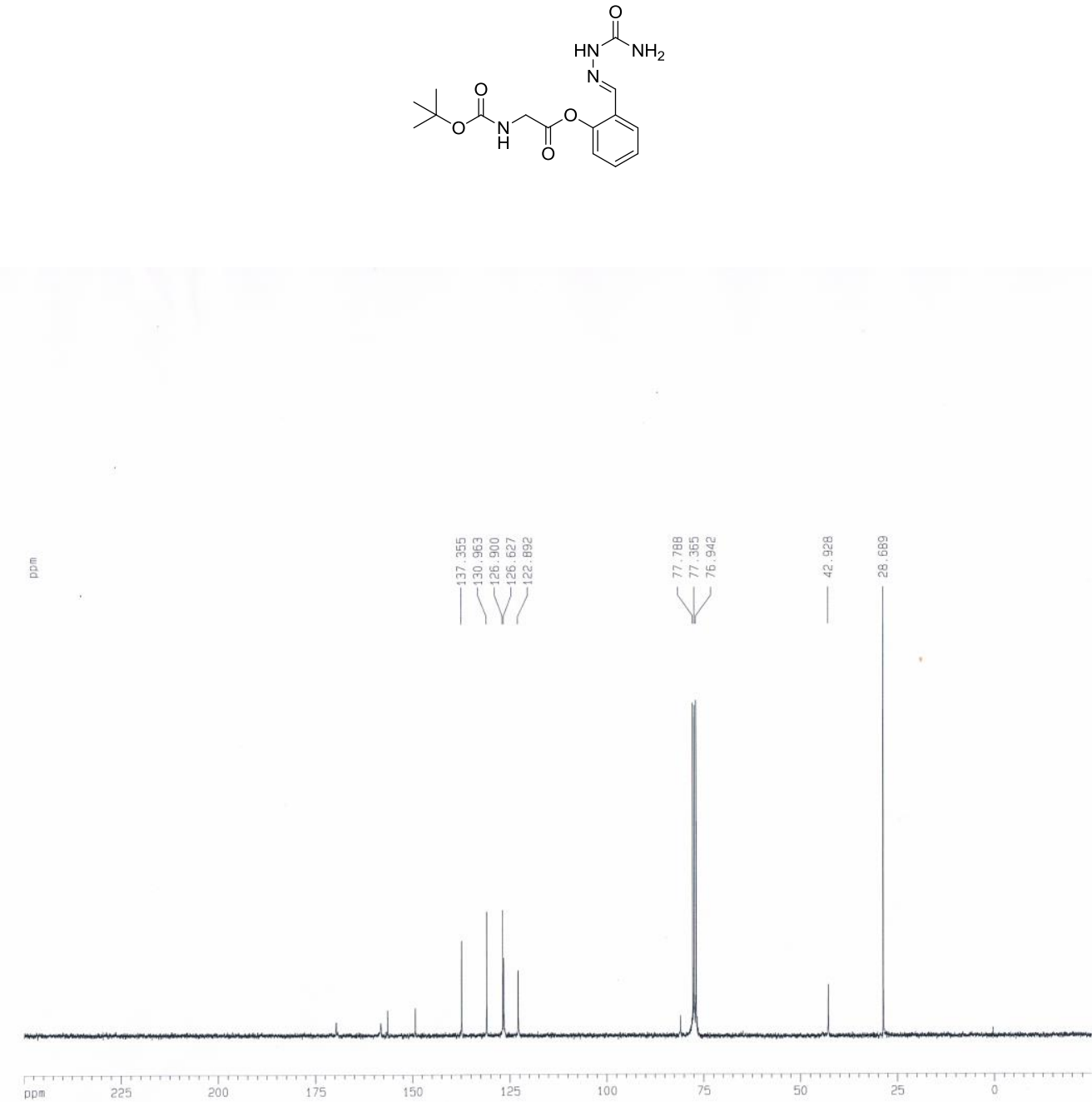
$\underline{{ }^{1} \mathrm{H} \text { spectrum of } B o c H N-L e u-C O-S A L^{\text {off }}}$
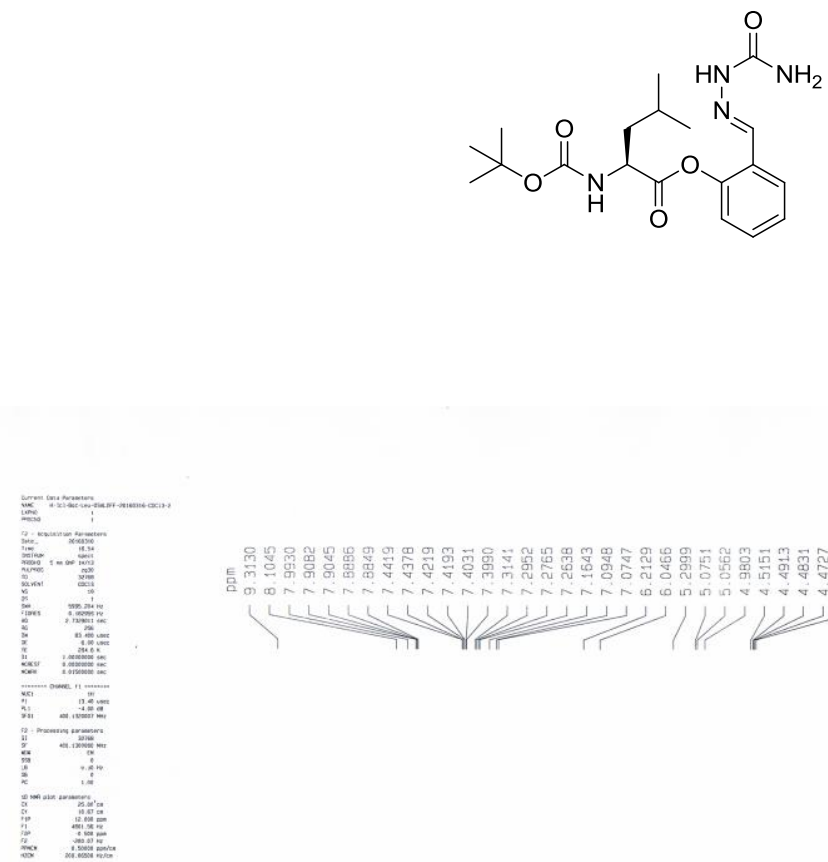

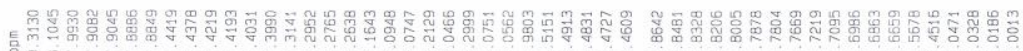

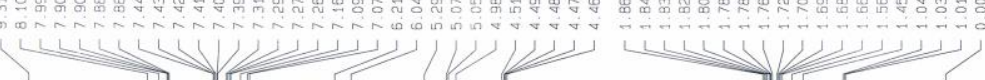

皮 


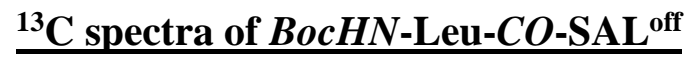
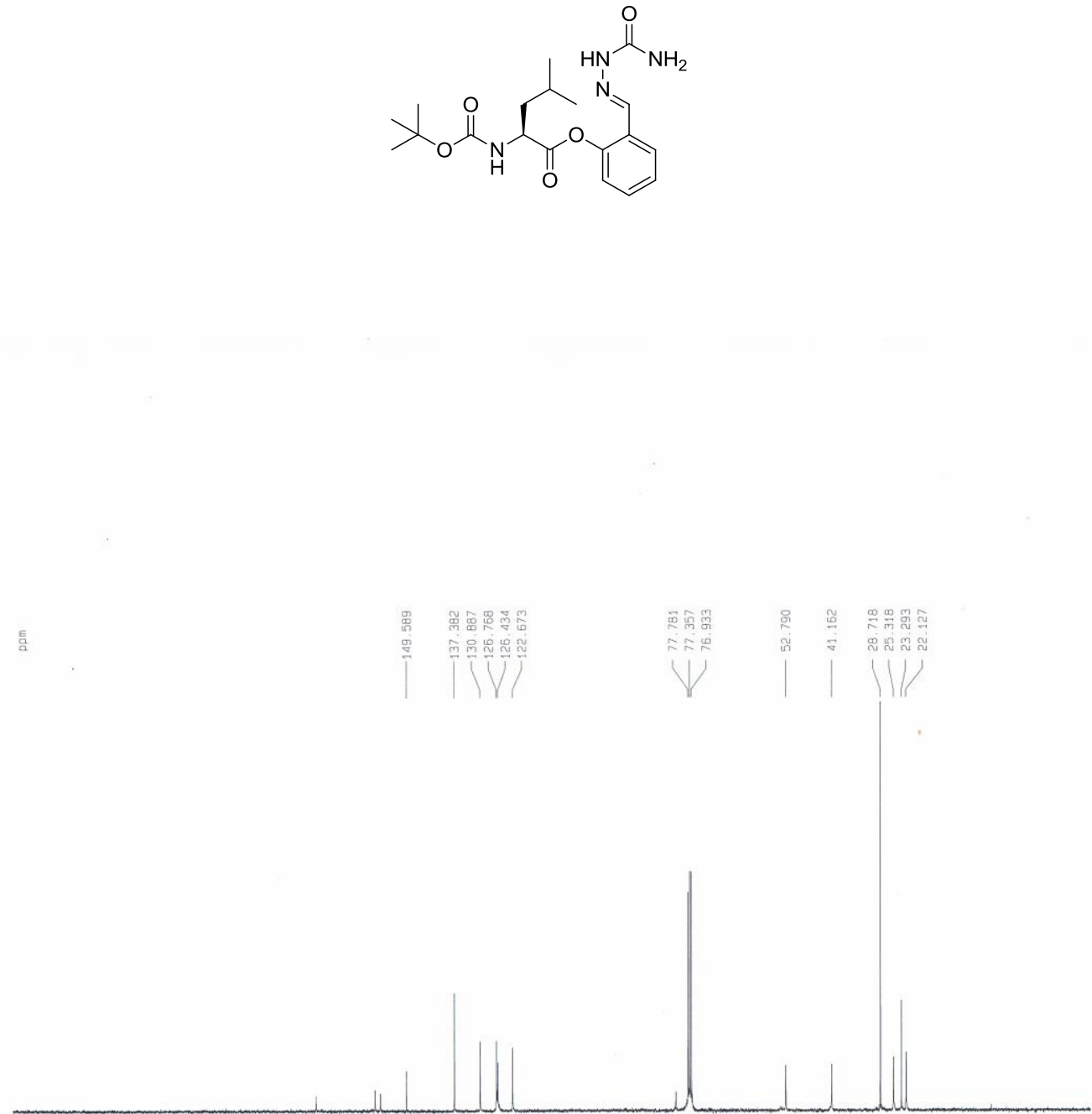
$\underline{{ }^{1} \mathrm{H} \text { spectrum of } B o c H N-M e t-C O-S A L^{\text {off }}}$

$>\underbrace{2}$

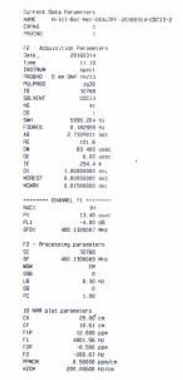

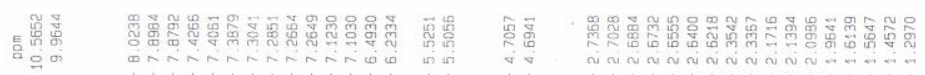
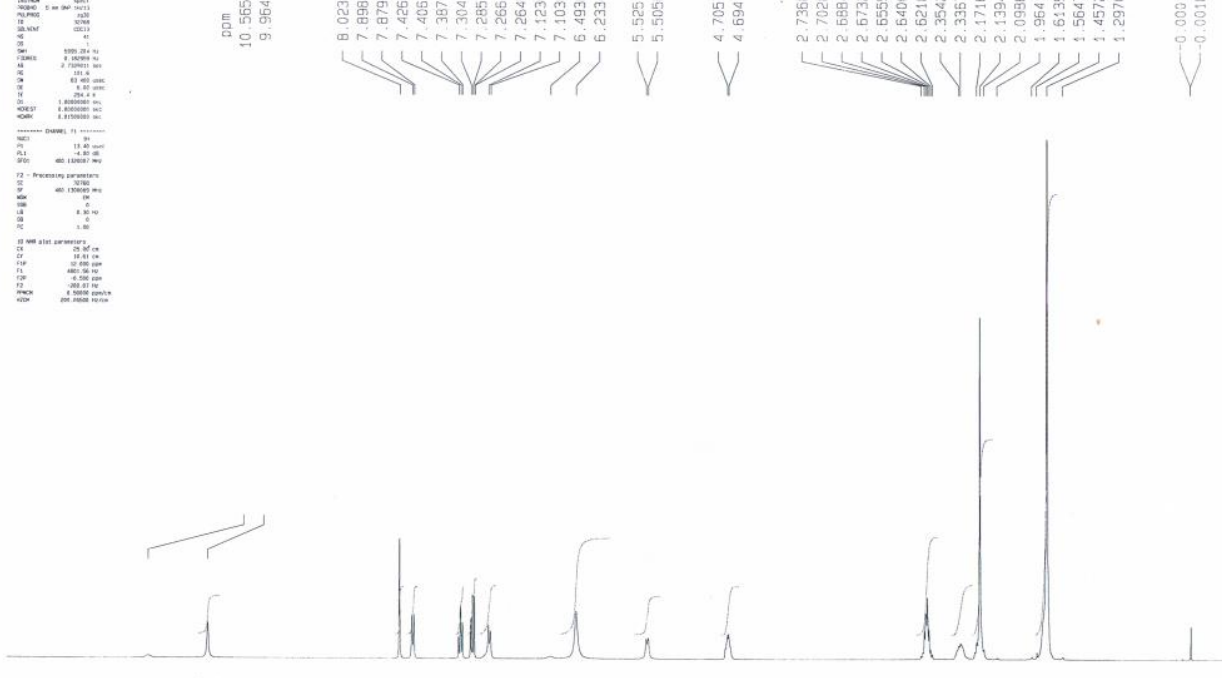

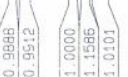

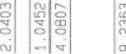


${ }^{13} \mathrm{C}$ spectrum of $\mathrm{BocHN}$-Met-CO-SAL ${ }^{\text {off }}$
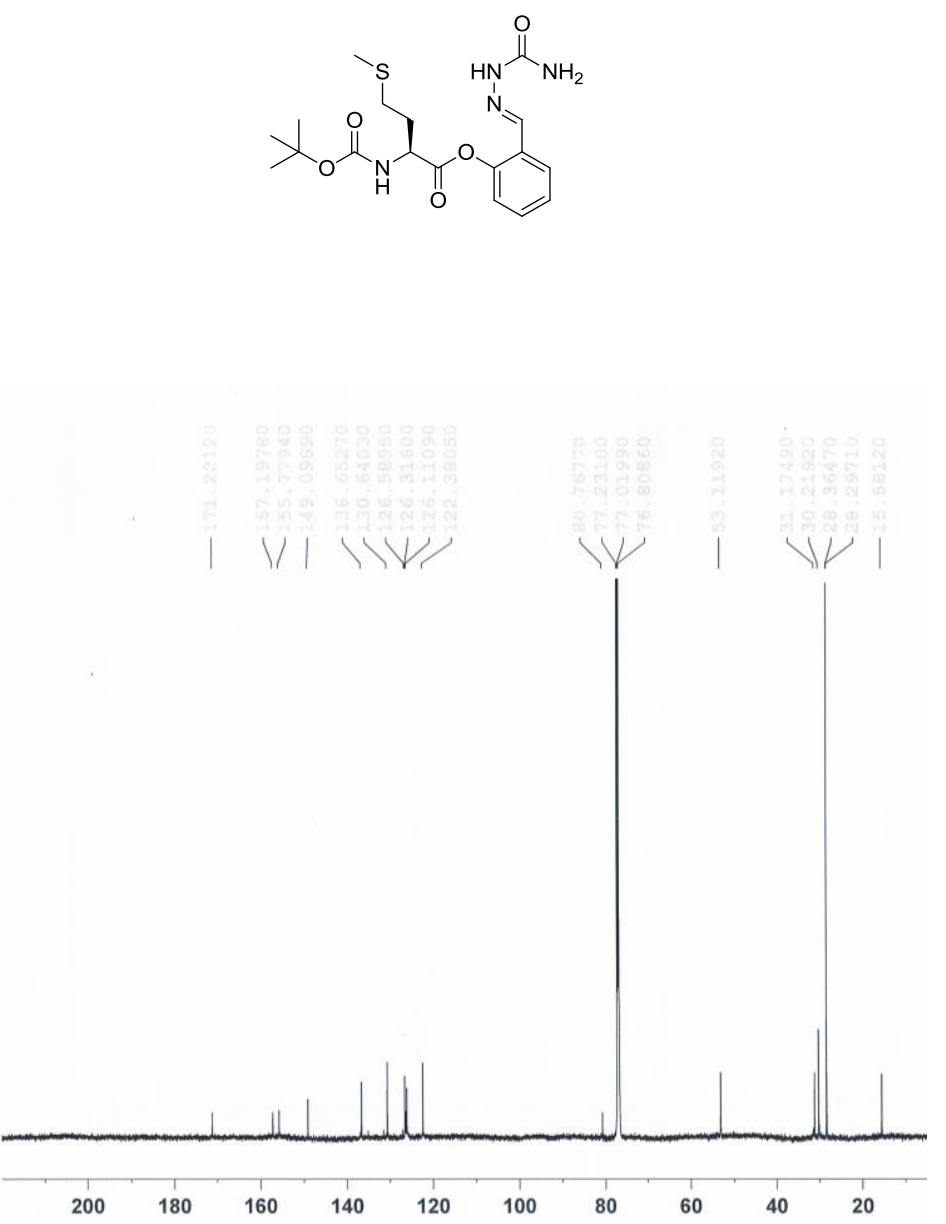


\section{$\underline{{ }^{1} \mathrm{H} \text { spectrum of } B o c H N-V \text { al-CO-SAL }}$}
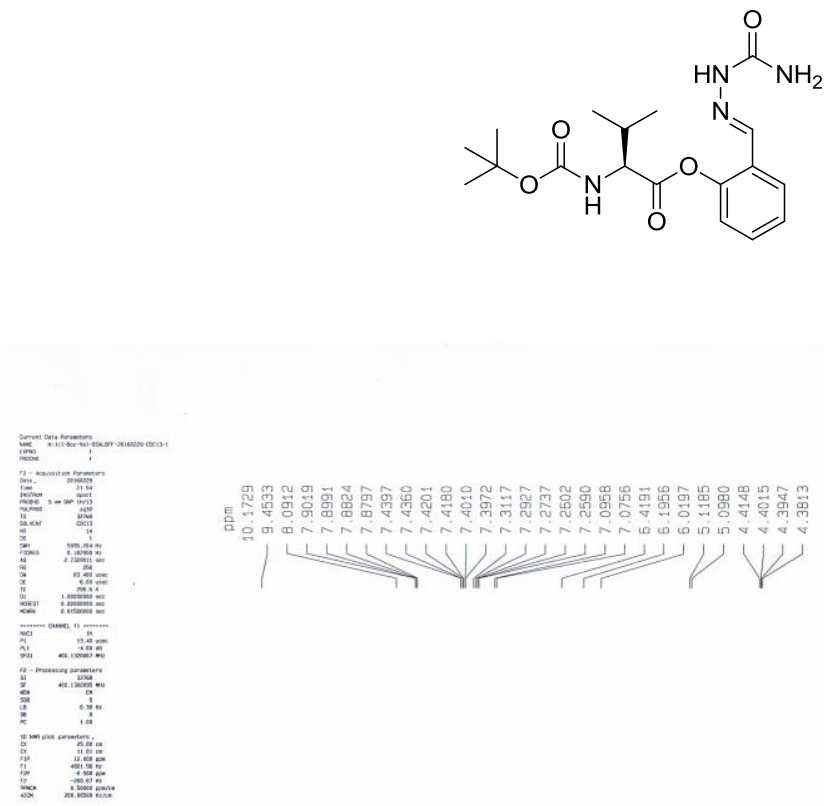

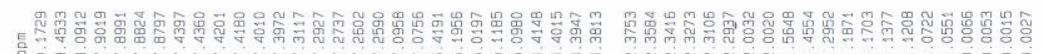
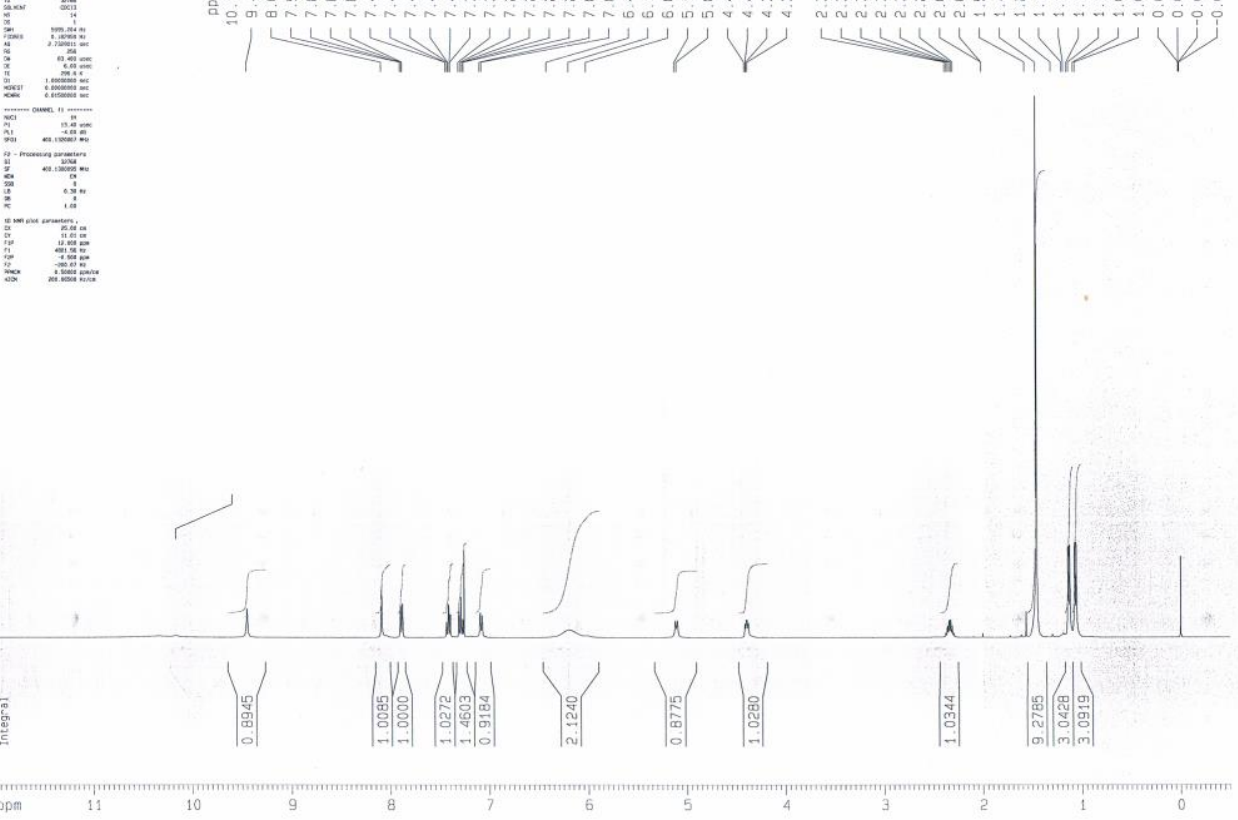
${ }^{13} \mathrm{C}$ spectrum of $\mathrm{BocHN}-\mathrm{Val}-\mathrm{CO}-\mathrm{SAL}{ }^{\text {off }}$
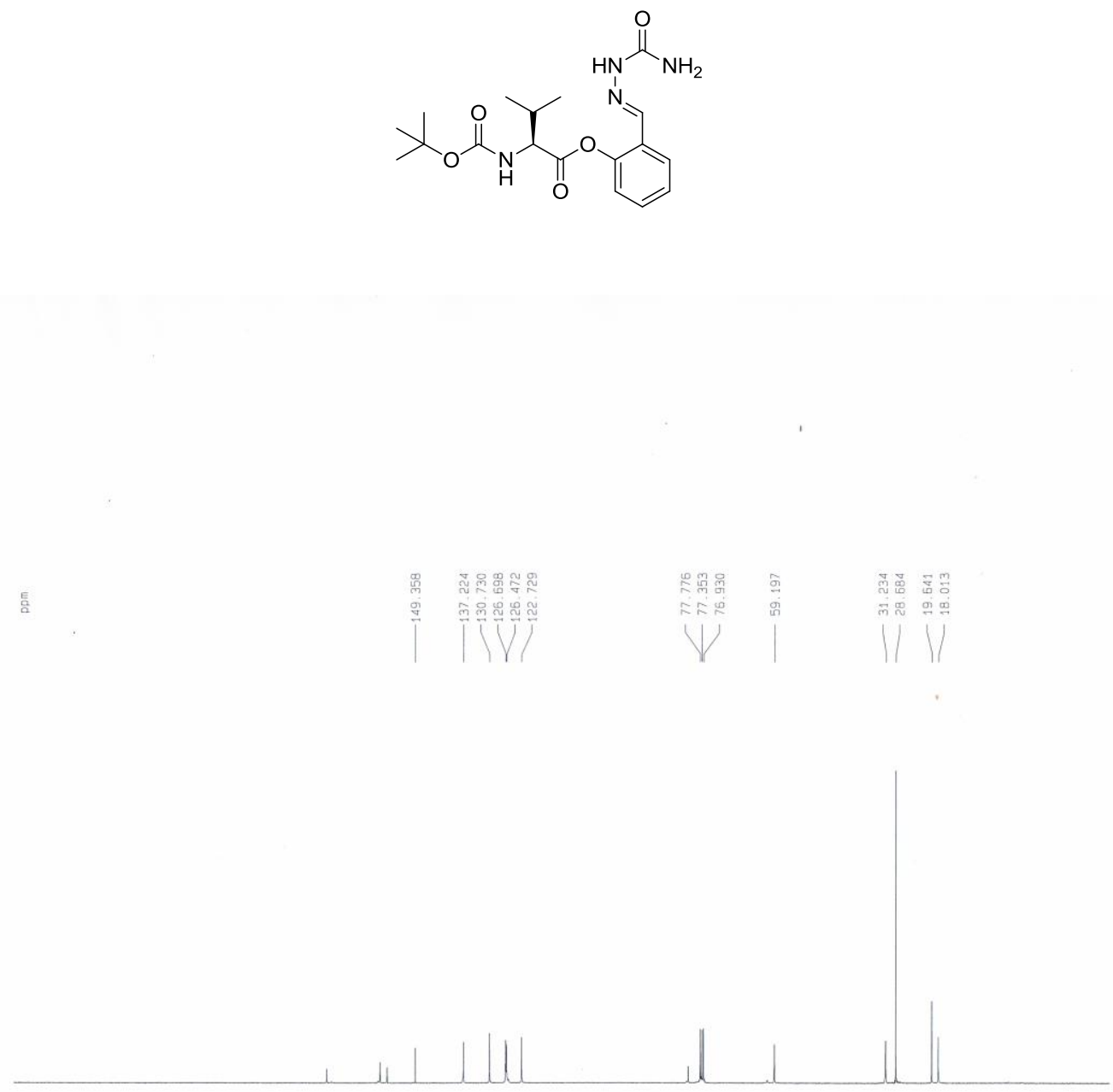


\section{$\underline{{ }^{1} \mathrm{H} \text { spectrum of } \mathrm{BocHN}-\mathrm{Tyr}\left(\mathrm{O}^{t} \mathrm{Bu}\right)-\mathrm{CO}-\mathrm{SAL}^{\text {off }}}$}
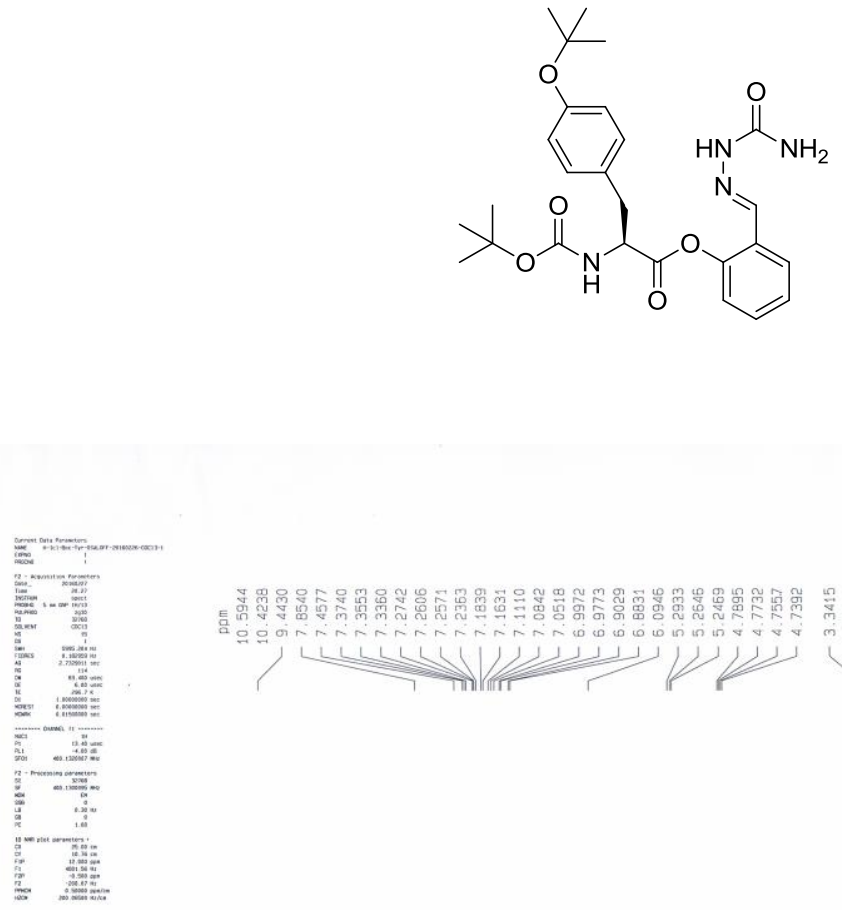

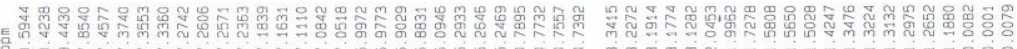

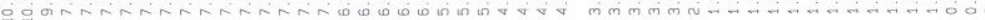
(

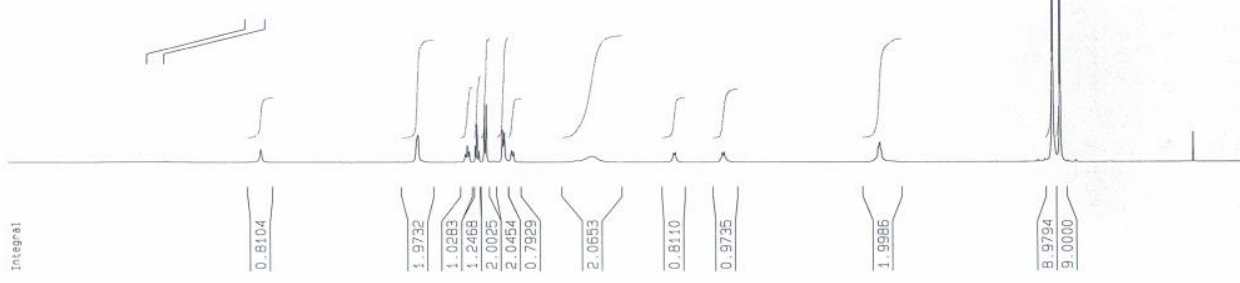


${ }^{13} \mathrm{C}$ spectrum of $\mathrm{BocHN}-\mathrm{Tyr}\left(\mathrm{O}^{t} \mathrm{Bu}\right)-\mathrm{CO}-\mathrm{SAL}^{\text {off }}$
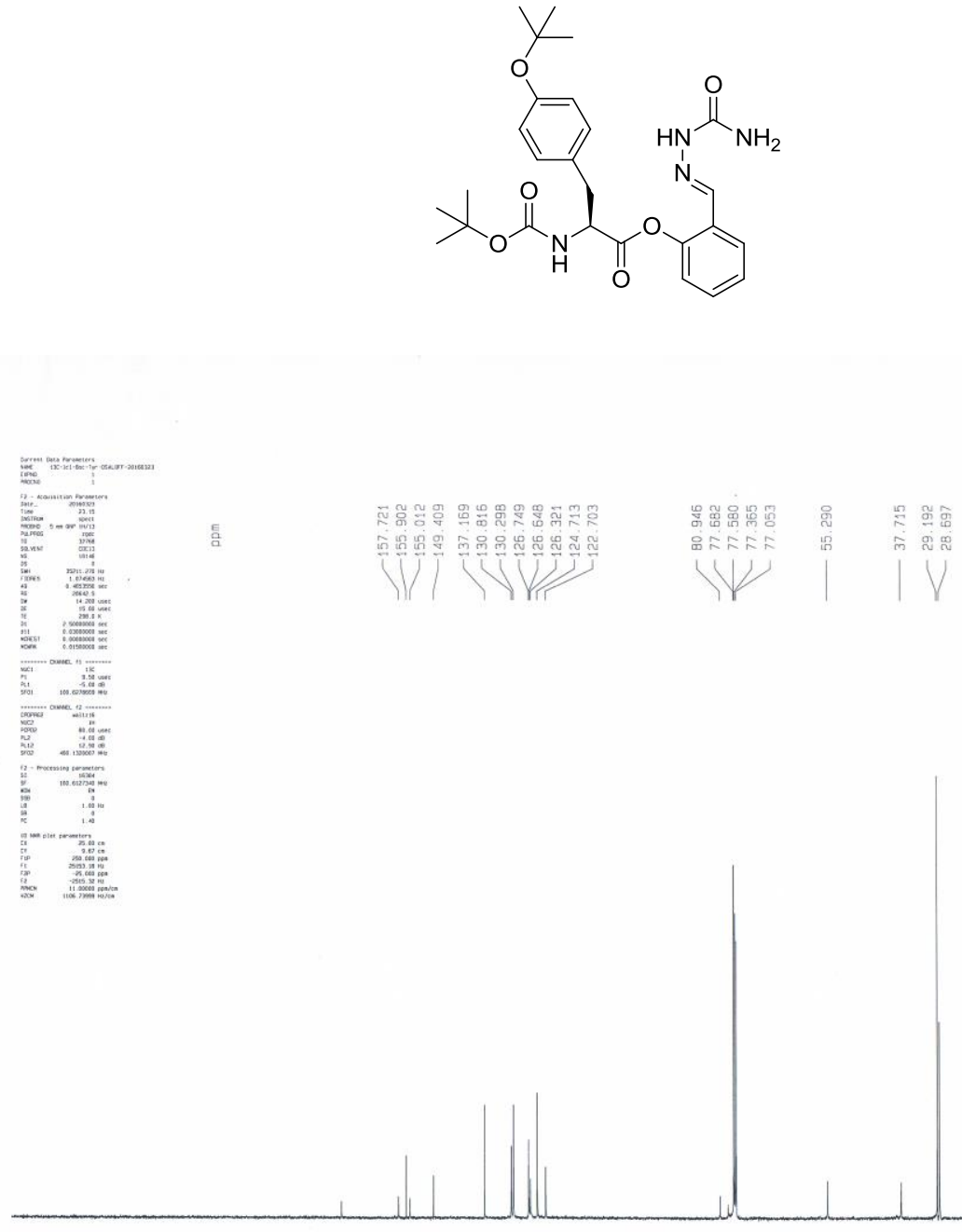


\section{$\underline{\text { XII. References }}$}

(1) (a) Kuroda, H.; Chen, Y. N.; Kimura, T.; Sakakibara S. Int. J. Pept. Protein Res. 1992, 40, 294-299. (b) Sakakibara S. Biopolymers 1995, 37, 17-28. (c) Li, H.; Jiang, X.; Ye, Y. H.; Fan, C.; Romoff T.; Goodman, M. Org. Lett. 1999, 1, 91-93. (d) Chen, J.; Chen, G.; Wu, B.; Wan, Q.; Tan, Z.; Hua, Z.; Danishefsky, S. J. Tetrahedron Lett. 2006, 47, 8013-8016. (e) Fernández-Tejada, A.; Vadola, P. A.; Danishefsky, S. J. J. Am. Chem. Soc. 2014, 136, 8450-8458.

(2) (a) Fang, G.-M.; Wang, J.-X.; Liu, L. Angew. Chem. Int. Ed. 2012, 51, 10347-10350. (b) Zheng, J.-S.; Tang, S.; Qi, Y.-K.; Wang, Z.-P. Liu, L.; Nature Protocols 2013, 8, 2483-2495.

(3) Lakshmi, G. J.; Kotra, S. R.; Peravali, J. B.; Kumar, P.P.B.S.; Surya, K. R.; Rao, S. Int. J. Bio-Sci. Bio-Tec. 2014, 6, 19-30.

(4) (a) Piontek, C.; Ring, P.; Harjes, O.; Heinlein, C.; Mezzato, S.; Lombana, N.; Pohner, C.; Puttner, M.; Silva, D. V.; Martin, A.; Schmid F. X.; Unverzagt, C. Angew. Chem. Int. Ed. 2009, 48, 1936-1940. (b) Piontek, C.; Silva, D. V.; Heinlein, C.; Pohner, C.; Mezzato, S.; Ring, P.; Martin, A.; Schmid, F. X.; Unverzagt, C. Angew. Chem. Int. Ed. 2009, 48, 1941-1945.

(5) Reif, A.; Siebenhaar, S.; Troster, A.; Schmalzlein, M.;Lechner, C.; Velisetty, P.;Gottwald, K.; Pohner, C.; Boos, I.; Schubert, V.; Rose-John, S.; Unverzagt, C. Angew. Chem. Int. Ed. 2014, 53, 12125-12131.

(6) Tang, S.; Si, Y.-Y.; Wang, Z.-P.; Mei, K.-R.; Chen, X.; Cheng, J.-Y.; Zheng, J.-S.; Liu, L. Angew. Chem. Int. Ed. 2015, 54, 5713-5717.

(7) Murakami, M.; Okamoto, R.; Izumi, M.; Kajihara, Y. Angew. Chem. Int. Ed. 2012, 51, 3567-3572. 